\title{
Ni-Catalyzed Reductive Coupling of Alkynes and Amides to Access Multi-Functionalized Indoles
}

\author{
Kwan Hong Min, Naeem Iqbal, and Eun Jin Cho* \\ Department of Chemistry, Chung-Ang University \\ 84 Heukseok-ro, Dongjak-gu, Seoul 06974, Republic of Korea \\ E-mail: ejcho@cau.ac.kr
}

\section{Supporting Information}

General Considerations

Additional Experiments

- Optimization of nickel-catalyzed arylative cyclization reaction (Table S1)

- gHMBC experiment (Figure S1)

- 1D NOESY experiment (Figure S2)

- Ni(0)-Mediated Catalytic Cycle (Figure S3)

- Reaction of phenylacetylene derivative in the presence of an alkyl silane (Scheme S1)

Experimental Details

Analytic Data for Synthesized Compounds

References 


\section{General Considerations}

\section{General Reagent Information}

All commercially available reagents including $\mathrm{Ni}(\mathrm{COD})_{2}, \quad \mathrm{Ni}(\mathrm{OAc})_{2} \cdot 4 \mathrm{H}_{2} \mathrm{O}, \quad 3-$ (diphenylphosphaneyl)propan-1-amine, arylboronic acid derivatives, and 2,2,2-trifluoroethanol were purchased from Sigma-Aldrich, Alfa Aesar, TCI or Strem Chemicals. All other reagents required for the synthesis of 2,2,2-trifluoro- $N$-methyl- $N$-(2-(prop-1-yn-1-yl)phenyl)acetamide derivative 1 were purchased from Sigma Aldrich, Alfa Aesar, or TCI chemical companies. Flash column chromatography was performed using Zeochem silica gel 60 (60-200 mesh).

\section{General Analytical Information}

The synthesized compounds including 2,2,2-trifluoro- $N$-methyl- $N$-(2-(prop-1-yn-1-yl)phenyl)acetamide derivatives (1) and 1-methyl-3-(1-phenylvinyl)-2-(trifluoromethyl)-1 $H$-indole derivatives (3) were characterized by ${ }^{1} \mathrm{H}$ NMR, ${ }^{13} \mathrm{C}$ NMR, and FT-IR spectroscopies, as well as mass spectrometry. Structural assignments were made with additional information from gHMBC and 1D NOESY experiments. NMR spectra were recorded on a Varian $600 \mathrm{MHz}$ instrument $\left(600 \mathrm{MHz}\right.$ for ${ }^{1} \mathrm{H} \mathrm{NMR}$ and $151 \mathrm{MHz}$ for ${ }^{13} \mathrm{C}$ NMR). Copies of ${ }^{1} \mathrm{H}$ NMR and ${ }^{13} \mathrm{C}$ NMR spectra can be found at the end of the Supporting Information. ${ }^{1} \mathrm{H}$ NMR chemical shifts are reported in parts per million (ppm) relative to residual chloroform (7.26 ppm) in the deuterated solvent. ${ }^{13} \mathrm{C}$ NMR spectra are reported in ppm relative to deuterochloroform (77.23 ppm) and all were obtained with ${ }^{1} \mathrm{H}$ decoupling. Coupling constants were reported in Hz. FT-IR spectra were recorded on a Nicolet 6700 FT-IR spectrometer (ThermoFisher). Reactions were monitored by thin layer chromatography and GC-MS using the Agilent GC 7890B/5977A inert MSD with Triple-Axis Detector. Mass spectral data of all unknown compounds were acquired at the Korea Basic Science Institute (Daegu) on a Jeol JMS 700 high-resolution mass spectrometer. A quadrupole mass analyzer was used for HRMS measurements. Melting points of unknown compounds were recorded on a Stuart SMP30 apparatus. 


\section{Additional Experiment}

Table S1. Optimization of nickel-catalyzed arylative cyclization reaction ${ }^{a, b}$

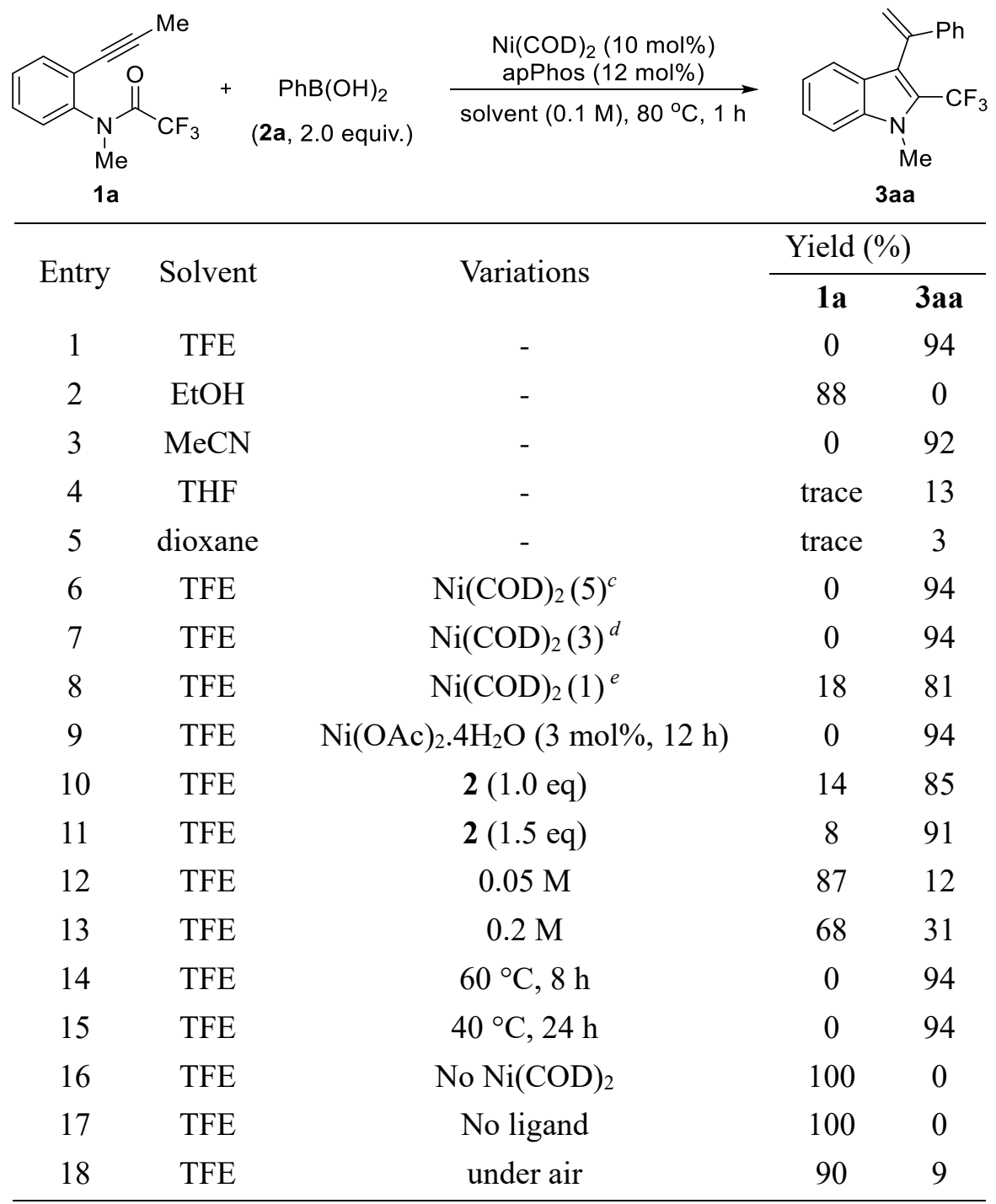

${ }^{a} \mathbf{1 a}$ at the $0.1 \mathrm{mmol}$ scale. ${ }^{b} \mathrm{GC}$ yield using dodecane as internal standard. ${ }^{c} 6 \mathrm{~mol} \%$ ligand was used.

${ }^{d} 4 \mathrm{~mol} \%$ ligand was used. ${ }^{e} 1.2 \mathrm{~mol} \%$ ligand was used. 
gHMBC experiment
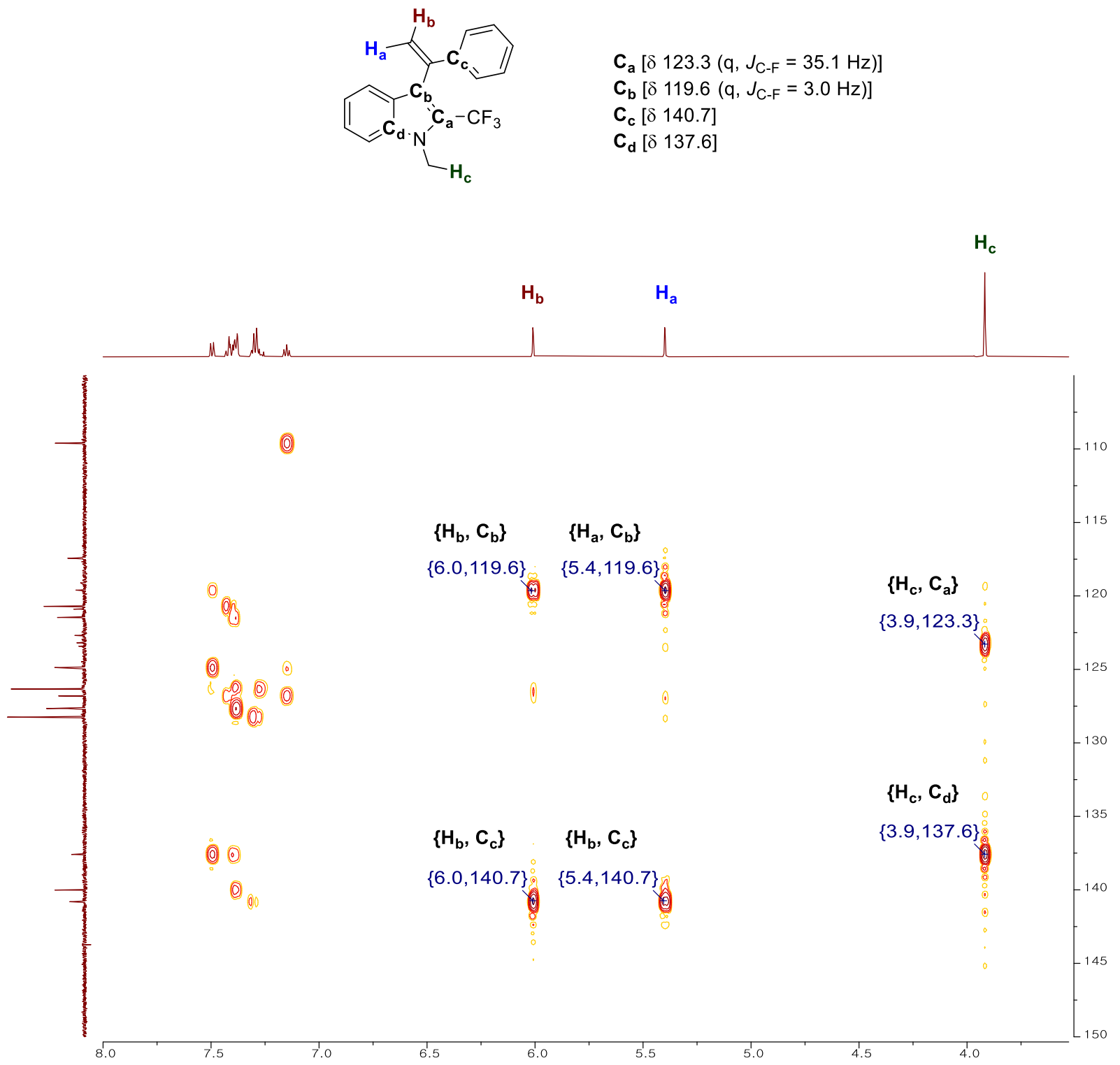

Figure S1. gHMBC spectrum of 3aa 


\section{D NOESY experiment}
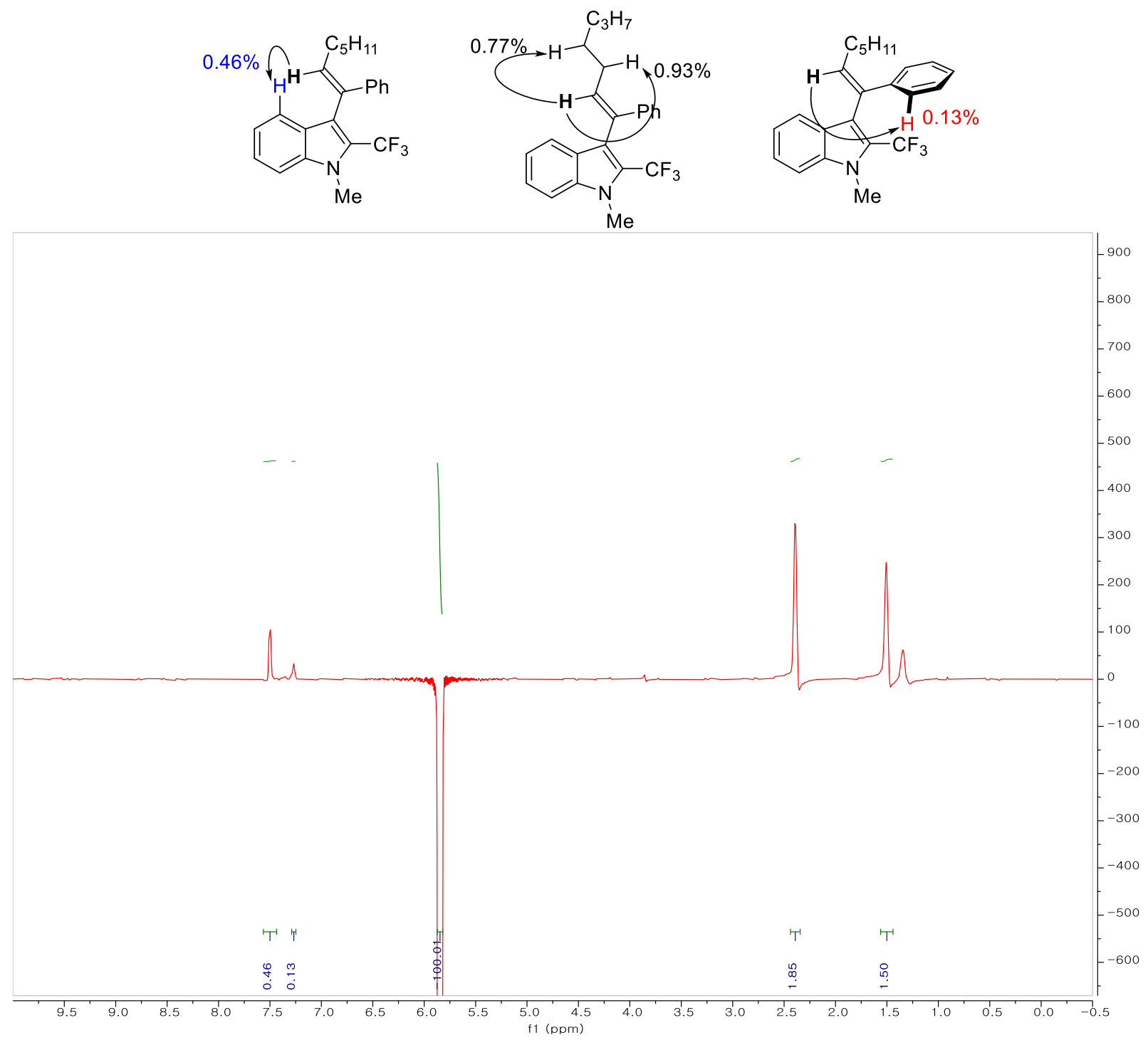

Figure S2. 1D NOESY spectrum of 3ha irradiated at $5.85 \mathrm{ppm}$ ( $E$-isomer: major) 


\section{Ni(0)-Mediated Catalytic Cycle}

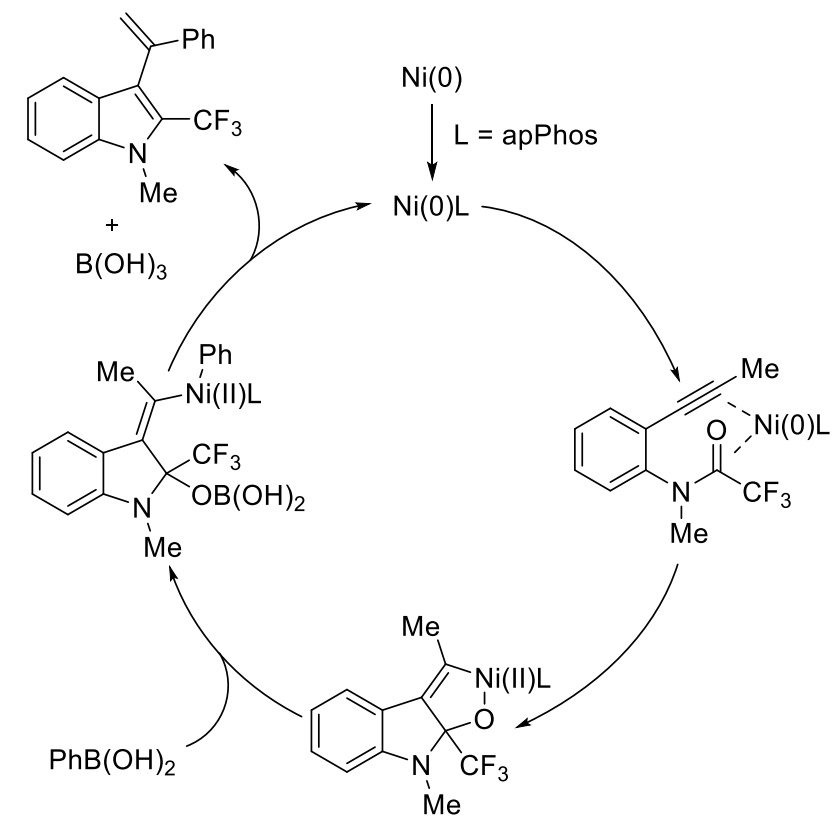

Figure S3. Proposed mechanism for the Ni(0)-mediated process

Coordination of an electron-rich alkyne and electron-deficient amide with $\mathrm{Ni}(0) \mathrm{L}$ leads to oxidative cyclization, resulting in the formation of a nickellacyle intermediate. This intermediate easily undergoes base-free transmetallation with an arylboronic acid, as the hemiaminal oxygen in the nickellacycle can act as an intrinsic base through the B-O interaction. The resulting vinyl nickel intermediate can easily undergo a $\mathrm{C}-\mathrm{C}$ bond forming reductive elimination followed by dehydration to yield the desired product along with the regeneration of the $\mathrm{Ni}(0)$ catalyst.

\section{Reaction of phenylacetylene derivative in the presence of an alkyl silane}

The reaction of phenylacetylene derivative did not proceed under the standard conditions, but in the presence of triethylsilane, the exocyclic alkene product $\mathbf{A}$ was observed, supporting the proposed $\mathrm{Ni}(0)$ catalyzed mechanism.
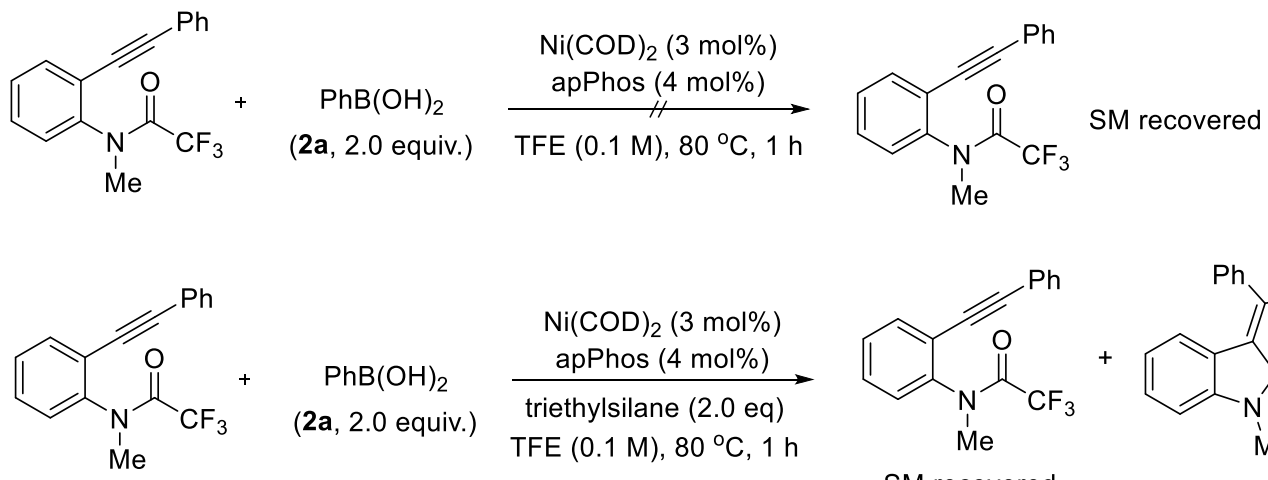

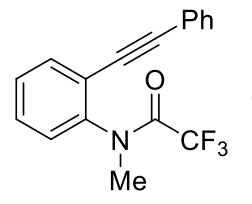

SM recovered $(\sim 80 \%)$<smiles>CCC1(C(F)(F)F)C(=C(c2ccccc2)c2ccccc2)c2ccccc2N1C</smiles>

A $(10 \%)$ not detected

Scheme S1. Reaction of phenylacetylene derivative 


\section{Experimental Details}

\section{General scheme for the preparation of 2,2,2-trifluoro-N-methyl-N-(2-(prop-1-yn-1- yl)phenyl)acetamide $1 \mathrm{a}-1 \mathrm{l}^{\mathrm{S1}}$}

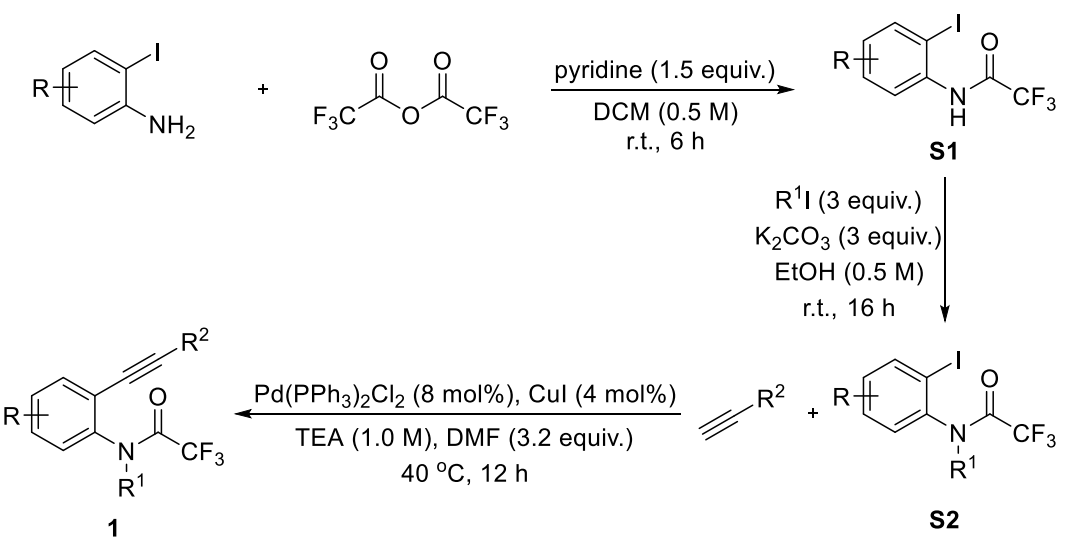

General experimental procedure for the preparation of 2,2,2-trifluoro- $N$-(2iodophenyl)acetamide derivative S1: An oven-dried 100-mL round-bottom flask equipped with a magnetic stir bar was charged with the 2-iodoaniline $(10 \mathrm{mmol})$, pyridine (1.5 equiv., $15 \mathrm{mmol})$ and $\mathrm{CH}_{2} \mathrm{Cl}_{2}(0.5 \mathrm{M}, 20 \mathrm{~mL})$. The solution was then cooled to $0{ }^{\circ} \mathrm{C}$ with the use of an ice bath, and 2,2,2trifluoroacetic anhydride (1.5 equiv., $15 \mathrm{mmol}$ ) was added dropwise to the reaction mixture. The reaction mixture was then taken out of the ice bath, and then allowed to warm to room temperature and stirred. After 6 hours, the reaction mixture was diluted with $\mathrm{CH}_{2} \mathrm{Cl}_{2}$ and washed with brine. The combined organic layer was dried over anhydrous $\mathrm{MgSO}_{4}$ and concentrated in vaccuo. The crude 2,2,2-trifluoro- $N$ (2-iodophenyl)acetamide was used without further purification as a starting material of the following step.

General experimental procedure for the preparation of 2,2,2-trifluoro- $N$-(2-iodophenyl)- $N$ -

alkylacetamide derivative S2: An oven-dried 100-mL round-bottom flask equipped with a magnetic stir bar was charged with the 2,2,2-trifluoro- $N$-(2-iodophenyl)acetamide (crude, ca. $10 \mathrm{mmol}$ ), potassium carbonate (3.0 equiv., $30 \mathrm{mmol})$, alkyliodide (3.0 equiv., $30 \mathrm{mmol})$, and $\mathrm{EtOH}(0.5 \mathrm{M}, 20 \mathrm{~mL})$. The reaction mixture was stirred at room temperature $\left(20-22{ }^{\circ} \mathrm{C}\right)$. After 12 hours, the reaction mixture was diluted with $\mathrm{CH}_{2} \mathrm{Cl}_{2}$ and washed with brine. The organic layer was dried over anhydrous $\mathrm{MgSO}_{4}$ and concentrated in vaccuo. The crude product was purified by flash column chromatography using hexane/EtOAc as the eluent to afford the 2,2,2-trifluoro- $N$-(2-iodophenyl)- $N$-alkylacetamide $\mathbf{S 2}$.

General experimental procedure for the preparation of 2,2,2-trifluoro- $N$-alkyl- $N$-(2(alkynyl)phenyl)acetamide derivative 1: An oven-dried 100-mL round-bottom flask equipped with a magnetic stir bar was charged with the 2,2,2-trifluoro- $N$-(2-iodophenyl)- $N$-alkylacetamide $\mathbf{S 2}$ (10 $\mathrm{mmol}), \mathrm{Pd}\left(\mathrm{PPh}_{3}\right)_{2} \mathrm{Cl}_{2}$ (4 mol\%), $\mathrm{CuI}(8 \mathrm{~mol} \%)$, DMF (3.2 equiv., $\left.2.5 \mathrm{~mL}\right)$ and $\mathrm{Et}_{3} \mathrm{~N}(1.0 \mathrm{M}, 10 \mathrm{~mL})$. 
Argon was bubbled through the reaction mixture for $15 \mathrm{~min}$, and alkyne (1.5 equiv., $15 \mathrm{mmol}$ ) was added. The reaction flask was placed into a preheated oil bath at $40{ }^{\circ} \mathrm{C}$ with stirring. After $12 \mathrm{~h}$, saturated aqueous $\mathrm{NH}_{4} \mathrm{Cl} / \mathrm{NH}_{3}$ was added and was diluted with $\mathrm{CH}_{2} \mathrm{Cl}_{2}$ and washed with brine. The organic layer was dried over anhydrous $\mathrm{MgSO}_{4}$ and concentrated in vaccuo. The crude product was purified by flash column chromatography using hexane/EtOAc as the eluent to afford the derivative $\mathbf{1}$.

\section{General scheme for the preparation of 2,2,2-trifluoro- $N$-methyl- $N$-(2-(prop-1-yn-1- yl)phenyl)acetamide 1m-1r}

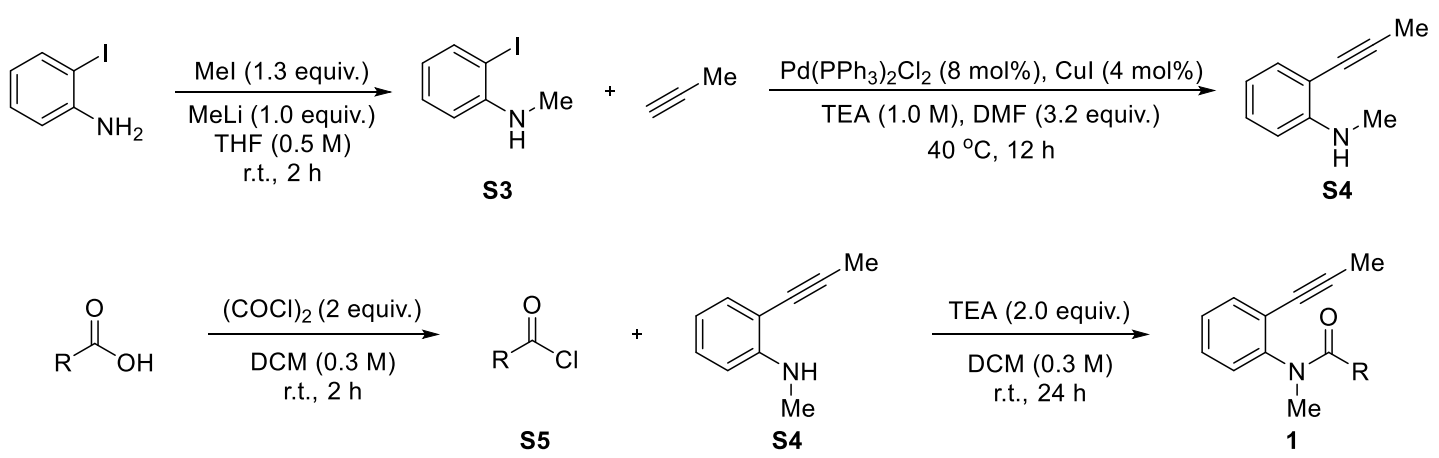

General experimental procedure for the preparation of 2-iodo- $N$-methylaniline $\mathrm{S3}^{\mathrm{S} 1}$ : An oven-dried 100-mL round-bottom flask equipped with a magnetic stir bar was charged with the 2iodoaniline $(50 \mathrm{mmol})$ and dry THF $(0.5 \mathrm{M}, 100 \mathrm{~mL})$ under Ar atmosphere. The solution was then cooled to $-78{ }^{\circ} \mathrm{C}$ with the use of a dry-ice bath, and $\mathrm{MeLi}$ (1.0 equiv., $1.1 \mathrm{M}$ in diethyl ether solution, $50 \mathrm{mmol}$ ) was added dropwise over 1 hour to the reaction mixture, and it was stirred for $30 \mathrm{~min}$ at $-78{ }^{\circ} \mathrm{C}$. Then, iodomethane (1.3 equiv., $66 \mathrm{mmol})$ in THF $(3.3 \mathrm{M}, 20.0 \mathrm{~mL})$ was added to the reaction mixture, and it was stirred for $1 \mathrm{~h}$ at $-78{ }^{\circ} \mathrm{C}$. Then, the flask was removed from the bath and allowed to stir at room temperature. After $2 \mathrm{~h}$, the reaction was quenched with saturated aqueous $\mathrm{NH}_{4} \mathrm{Cl} / \mathrm{NH}_{3}$, and the mixture was diluted with diethyl ether and washed with brine. The combined organic layer was dried over anhydrous $\mathrm{MgSO}_{4}$ and concentrated in vaccuo. The crude product was purified by flash column chromatography using hexane/EtOAc as the eluent to afford the 2-iodo- $N$-methylaniline $\mathbf{S 3}$.

\section{General experimental procedure for the preparation of $N$-methyl-2-(prop-1-yn-1-yl)aniline}

S4: An oven-dried 100-mL round-bottom flask equipped with a magnetic stir bar was charged with the 2iodo- $N$-methylaniline S3 (10 mmol), $\mathrm{Pd}\left(\mathrm{PPh}_{3}\right)_{2} \mathrm{Cl}_{2}(4 \mathrm{~mol} \%), \mathrm{CuI}(8 \mathrm{~mol} \%), \mathrm{DMF}(3.2 \mathrm{M}, 2.5 \mathrm{~mL})$ and $\mathrm{Et}_{3} \mathrm{~N}(1.0 \mathrm{M}, 10 \mathrm{~mL})$. Argon was bubbled through the reaction mixture for $15 \mathrm{~min}$, and propyne $(1.5$ equiv., $15 \mathrm{mmol}$ ) was added. The reaction flask was placed into a preheated oil bath at $40{ }^{\circ} \mathrm{C}$ with stirring. After $12 \mathrm{~h}$, saturated aqueous $\mathrm{NH}_{4} \mathrm{Cl} / \mathrm{NH}_{3}$ was added and was diluted with $\mathrm{CH}_{2} \mathrm{Cl}_{2}$ and washed with brine. The organic layer was dried over anhydrous $\mathrm{MgSO}_{4}$ and concentrated in vaccuo. The crude product was 
purified by flash column chromatography using hexane/EtOAc as the eluent to afford the $N$-methyl-2(prop-1-yn-1-yl)aniline $\mathbf{S 4}$.

\section{General experimental procedure for the preparation of $N$-methyl- $N$-(2-(prop-1-yn-1-} yl)phenyl)acetamide derivative $1^{\mathrm{S} 2}$ : An oven-dried $100-\mathrm{mL}$ round-bottom flask equipped with a magnetic stir bar was charged with the carboxylic acid $(10 \mathrm{mmol})$, DMF $(10 \mathrm{M}, 1 \mathrm{~mL})$ and $\operatorname{dry~} \mathrm{CH}_{2} \mathrm{Cl}_{2}$ $(0.3 \mathrm{M}, 30 \mathrm{~mL})$ under Ar atmosphere. The solution was then cooled to $0{ }^{\circ} \mathrm{C}$ with the use of an ice bath, and $(\mathrm{COCl})_{2}\left(2.0\right.$ equiv.) was added dropwise, and it was stirred for $10 \mathrm{~min}$ at $0{ }^{\circ} \mathrm{C}$. Then, the flask was removed from the ice bath and allowed to stir at room temperature. After 1 h, the crude acyl chloride S5 was concentrated in vaccuo and used without further purification as a starting material of the following step. An oven-dried 100-mL round-bottom flask equipped with a magnetic stir bar was charged with the $N$-methyl-2-(prop-1-yn-1-yl)aniline $\mathbf{S 4}$ (1 mmol), Et $3 \mathrm{~N}$ (2.0 equiv., $2 \mathrm{mmol}$ ) and $\mathrm{CH}_{2} \mathrm{Cl}_{2}(0.3 \mathrm{M}, 30 \mathrm{~mL}$ ) under Ar atmosphere. The solution was then cooled to $0{ }^{\circ} \mathrm{C}$ with the use of an ice bath, and the crude acyl chloride $\mathbf{S 5}$ in $\mathrm{CH}_{2} \mathrm{Cl}_{2}$ was added dropwise, and it was stirred for $10 \mathrm{~min}$ at $0{ }^{\circ} \mathrm{C}$. Then, the flask was removed from the ice bath and allowed to stir at room temperature. After $24 \mathrm{~h}$, the reaction was quenched with saturated $\mathrm{NaHCO}_{3}$, and the mixture was diluted with $\mathrm{CH}_{2} \mathrm{Cl}_{2}$ and washed with brine. The combined organic layer was dried over anhydrous $\mathrm{MgSO}_{4}$ and concentrated in vaccuo. The crude product was purified by flash column chromatography using hexane/EtOAc as the eluent to afford the $N$-methyl- $N$-(2(prop-1-yn-1-yl)phenyl)acetamide derivative $\mathbf{1}$.

\section{General Scheme for the synthesis of indole derivative 3:}
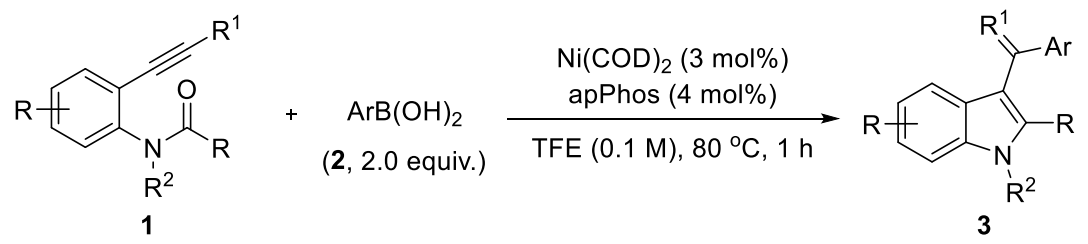

In an argon-filled glovebox, an oven-dried $20-\mathrm{mL}$ resealable reaction tube equipped with a magnetic stir bar was charged with 2-alkynylphenyl trifluoroacetamide derivative $1(0.5 \mathrm{mmol})$, arylboronic acid, 2 (2.0 equiv., $1 \mathrm{mmol}), \mathrm{Ni}(\mathrm{COD})_{2}(3 \mathrm{~mol} \%)$, apPhos $(4 \mathrm{~mol} \%)$, and TFE $(0.1 \mathrm{M}, 5 \mathrm{~mL})$. The reaction tube was then sealed with a screw-cap, taken out of the glove box, and placed into a preheated oil bath at $80{ }^{\circ} \mathrm{C}$ with stirring. After $1 \mathrm{~h}$, the tube was removed from the oil bath and allowed to cool to room temperature. The reaction mixture was concentrated in vaccuo, and purified by flash column chromatography using hexane/EtOAc as the eluent to afford the corresponding indole product derivative $\mathbf{3}$. 


\section{Synthesis of 1-methyl-3-(1-phenylvinyl)-2-(trifluoromethyl)-1H-indole (3aa) on 1 mmol- scale}

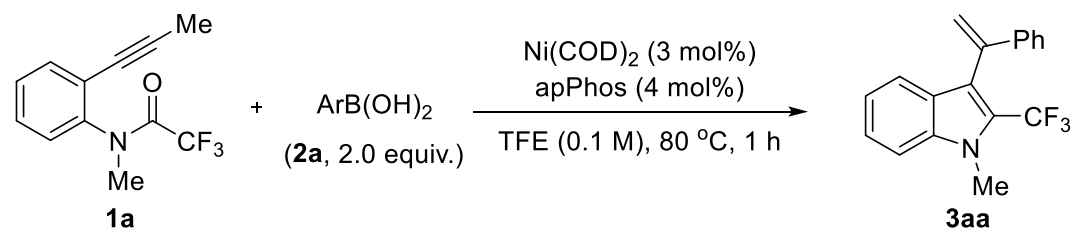

In an argon-filled glovebox, an oven-dried $20-\mathrm{mL}$ resealable reaction tube equipped with a magnetic stir bar was charged with 2,2,2-trifluoro-N-methyl-N-(2-(prop-1-yn-1-yl)phenyl)acetamide 1a (1 mmol, 240 $\mathrm{mg}$ ), phenylboronic acid, 2a (2.0 equiv., $2 \mathrm{mmol}), \mathrm{Ni}(\mathrm{COD})_{2}$ (3 mol\%), apPhos (4 mol\%), and TFE (0.1 $\mathrm{M}, 10 \mathrm{~mL}$ ). The reaction tube was then sealed with a screw-cap, taken out of the glove box, and placed into a preheated oil bath at $80{ }^{\circ} \mathrm{C}$ with stirring. After $1 \mathrm{~h}$, the tube was removed from the oil bath and allowed to cool to room temperature. The reaction mixture was concentrated in vaccuo, and purified by flash column chromatography using hexane/EtOAc as the eluent to afford the corresponding indole product 3aa (274 mg, 91\%).

\section{Synthesis of 1-methyl-3-(1-phenylvinyl)-2-(trifluoromethyl)-1H-indole (3aa) on 5 mmol-} scale
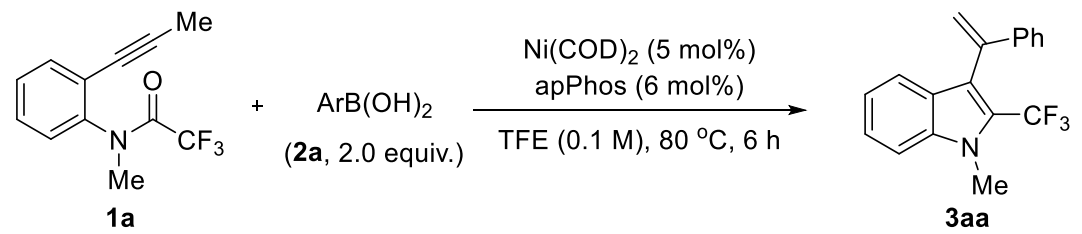

In an argon-filled glovebox, an oven-dried 100-mL round-bottom flask equipped with a magnetic stir bar was charged with 2,2,2-trifluoro-N-methyl-N-(2-(prop-1-yn-1-yl)phenyl)acetamide 1a (5 mmol, $1.21 \mathrm{~g})$, phenylboronic acid, $2 \mathbf{a}$ (2.0 equiv., $10 \mathrm{mmol}), \mathrm{Ni}(\mathrm{COD})_{2}(5 \mathrm{~mol} \%)$, apPhos $(6 \mathrm{~mol} \%)$, and TFE $(0.1 \mathrm{M}$, $50 \mathrm{~mL}$ ). The flask was then sealed with a rubber-cap, taken out of the glove box, and placed into a preheated oil bath at $80{ }^{\circ} \mathrm{C}$ with stirring. After $6 \mathrm{~h}$, the flask was removed from the oil bath and allowed to cool to room temperature. The reaction mixture was concentrated in vaccuo, and purified by flash column chromatography using hexane/EtOAc as the eluent to afford the corresponding indole product 3aa (1.28 g, $85 \%)$. 


\section{General Scheme for the preparation of $\mathbf{C F}_{3}$ analogue of pravadoline 7:}
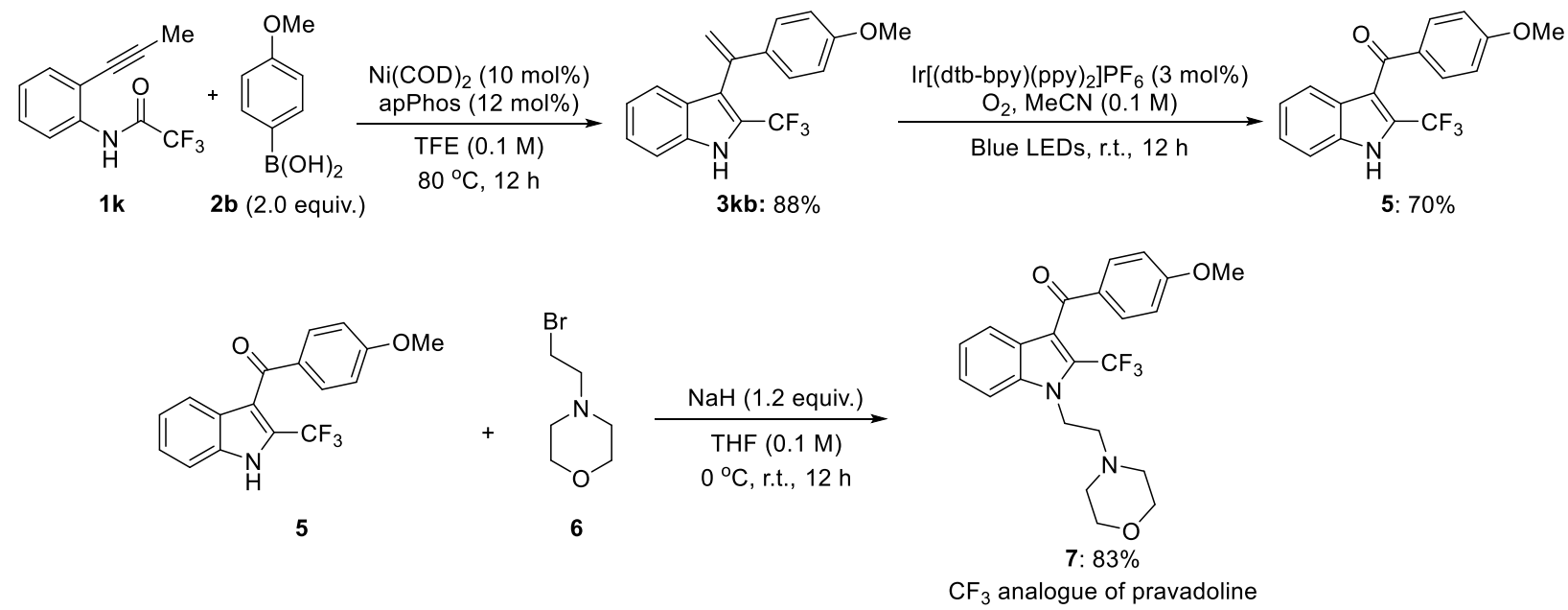

Experimental procedure for the synthesis of 3kb: In an argon-filled glovebox, an oven-dried 30$\mathrm{mL}$ resealable reaction tube equipped with a magnetic stir bar was charged with $\mathbf{1 k}(1 \mathrm{mmol}, 227 \mathrm{mg})$ and 2b (2.0 equiv., $2 \mathrm{mmol})$. $\mathrm{Ni}(\mathrm{COD})_{2}(10 \mathrm{~mol} \%)$, apPhos $(12 \mathrm{~mol} \%)$ and TFE $(0.1 \mathrm{M}, 10 \mathrm{~mL})$ were added to it. The reaction tube was then sealed with a screw-cap, taken out of the glove box, and placed into a preheated oil bath at $80{ }^{\circ} \mathrm{C}$ with stirring. After $12 \mathrm{~h}$, the tube was removed from the oil bath and allowed to cool to room temperature. The reaction mixture was concentrated in vaccuo, and purified by flash column chromatography using hexane/EtOAc as the eluent to afford 3kb (280 mg, 88\%).

Experimental procedure for the synthesis of 5: An oven-dried 10-mL reaction tube equipped with a magnetic stir bar was charged with $3 \mathbf{k b}(0.5 \mathrm{mmol}, 160 \mathrm{mg}), \operatorname{Ir}\left[(\mathrm{dtb}-\mathrm{bpy})_{2}\right] \mathrm{PF}_{6}(3 \mathrm{~mol} \%)$, and $\mathrm{MeCN}$ $(0.1 \mathrm{M}, 5 \mathrm{~mL})$. Molecular oxygen was bubbled through the reaction mixture for $2 \mathrm{~min}$, and the tube was sealed with a silicone septum screw cap. An oxygen-filled balloon was fixed to the reaction tube and placed under $450 \mathrm{~nm}$ blue LEDs at room temperature $\left(20-22{ }^{\circ} \mathrm{C}\right)$. After $12 \mathrm{~h}$, the reaction mixture was concentrated in vaccuo, and purified by flash column chromatography using hexane/EtOAc as the eluent to afford the carbonyl compound 5 (112 $\mathrm{mg}, 70 \%)$.

Experimental procedure for the synthesis of 7: An oven-dried 50-mL round-bottom flask equipped with a magnetic stir bar was charged with $5(1 \mathrm{mmol}, 320 \mathrm{mg})$ in dried THF $(0.2 \mathrm{M}, 5 \mathrm{~mL})$ under Ar atmosphere. The solution was then cooled to $0{ }^{\circ} \mathrm{C}$ with the use of an ice bath, and $\mathrm{NaH}$ (1.2 equiv., 1.2 mmol) was added to the reaction mixture, and it was stirred for $30 \mathrm{~min}$ at $0{ }^{\circ} \mathrm{C}$. Then, 4-(2Bromoethyl)morpholine 6 ( 1.5 equiv., $1.5 \mathrm{mmol}$ ) was added to the reaction mixture, and it was stirred for 30 min at $0{ }^{\circ} \mathrm{C}$. Then, the flask was removed from the ice bath and allowed to stir at room temperature. After $12 \mathrm{~h}$, the reaction mixture was concentrated in vaccuo, and purified by flash column chromatography using hexane/EtOAc as the eluent to afford the corresponding indole product $\mathrm{CF}_{3}$ analogue of pravadoline 7 (360 $\mathrm{mg}, 83 \%)$. 


\section{General Scheme for the synthesis of 9:}

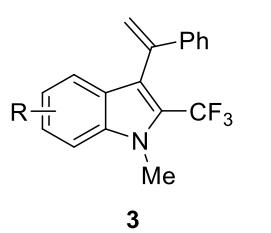

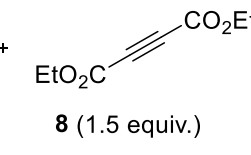

toluene $(0.1 \mathrm{M})$
$110^{\circ} \mathrm{C}, 60 \mathrm{~h}$

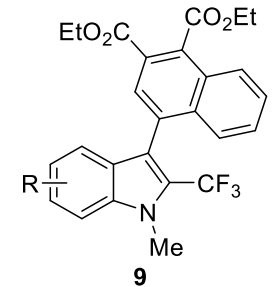

An oven-dried 10-mL reaction tube equipped with a magnetic stir bar was charged with 3 (0.2 mmol), diethyl acetylenedicarboxylate (1.5 equiv., $0.3 \mathrm{mmol})$, and toluene $(0.1 \mathrm{M}, 2 \mathrm{~mL})$. The reaction tube was placed into a preheated oil bath at $110{ }^{\circ} \mathrm{C}$ with stirring. After $60 \mathrm{~h}$, the tube was removed from the oil bath and allowed to cool to room temperature. The reaction mixture was concentrated in vaccuo, and purified by flash column chromatography using hexane/EtOAc as the eluent to afford the corresponding indole product derivative 9 .

\section{Analytic Data for Synthesized Compounds}

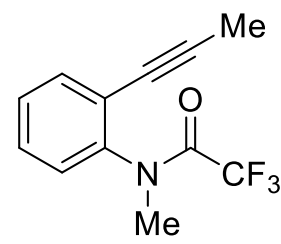

$1 a$ (2,2,2-trifluoro- $N$-methyl- $N$-(2-(prop-1-yn-1-yl)phenyl)acetamide (from $10 \mathrm{mmol}$ of S2 to 1a, $2.15 \mathrm{~g}, 89 \%)$ : pale yellow liquid; ${ }^{1} \mathbf{H}$ NMR (600 $\left.\mathbf{~ M H z}, \mathbf{C D C l}_{3}\right) \delta 7.49-$ $7.45(\mathrm{~m}, 1 \mathrm{H}), 7.34-7.31(\mathrm{~m}, 2 \mathrm{H}), 7.21(\mathrm{~d}, J=7.8 \mathrm{~Hz}, 1 \mathrm{H}), 3.33(\mathrm{~s}, 3 \mathrm{H}), 2.04(\mathrm{~s}, 3 \mathrm{H})$;

${ }^{13} \mathbf{C}$ NMR (151 MHz, $\left.\mathbf{C D C l}_{3}\right) \delta 157.4\left(\mathrm{q},{ }^{2} J_{C-F}=35.7 \mathrm{~Hz}\right), 141.9,133.3,129.2,128.6$, $128.3\left(\mathrm{q},{ }^{4} J_{C-F}=1.5 \mathrm{~Hz}\right), 123.8,116.5\left(\mathrm{q},{ }^{1} J_{C-F}=288.2 \mathrm{~Hz}\right), 92.4,75.1,384,4.6$; IR (neat): $v_{\max }=2922$, 2235, 1701, 1491, 1199, 1153, $753 \mathrm{~cm}^{-1}$; HRMS m/z (EI) calc. for $\mathrm{C}_{12} \mathrm{H}_{10} \mathrm{~F}_{3} \mathrm{NO}\left[\mathrm{M}^{+}\right]$241.0714, found 241.0716; $\mathbf{R}_{f} 0.53$ (hexane/EtOAc, 4/1).

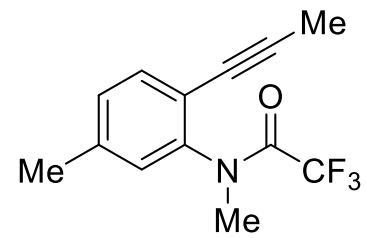

$1 b \quad$ (2,2,2-trifluoro- $N$-methyl- $N$-(5-methyl-2-(prop-1-yn-1-yl)phenyl)acetamide (from $5.0 \mathrm{mmol}$ of $\mathbf{S 2}$ to $\mathbf{1 b}, 1.10 \mathrm{~g}, 86 \%$ ): orange liquid; ${ }^{\mathbf{1}} \mathbf{H}$ NMR (600 $\mathbf{M H z}$, $\left.\mathbf{C D C l}_{3}\right) \delta 7.34(\mathrm{~d}, J=7.9 \mathrm{~Hz}, 1 \mathrm{H}), 7.12(\mathrm{dd}, J=7.9,1.7 \mathrm{~Hz}, 1 \mathrm{H}), 7.01(\mathrm{~s}, 1 \mathrm{H})$, 3.31 (s, 3H), 2.35 (s, 3H), $2.02(\mathrm{~s}, 3 \mathrm{H}) ;{ }^{13} \mathbf{C}$ NMR (151 MHz, CDCl $) \delta 157.4(\mathrm{q}$, $\left.{ }^{2} J_{C-F}=35.8 \mathrm{~Hz}\right), 141.8,139.1,132.9,129.9,128.7\left(\mathrm{q},{ }^{4} J_{C-F}=1.4 \mathrm{~Hz}\right), 120.6,116.5\left(\mathrm{q},{ }^{1} J_{C-F}=288.1 \mathrm{~Hz}\right)$, 91.3, 75.0, 38.4, 21.3, 4.5; IR (neat): $v_{\max }=2922,2236,1697,1222,1180,1150,736 \mathrm{~cm}^{-1} ; \mathbf{H R M S ~ m} / \mathrm{z}$ (EI) calc. for $\mathrm{C}_{13} \mathrm{H}_{12} \mathrm{~F}_{3} \mathrm{NO}\left[\mathrm{M}^{+}\right] 255.0871$, found 255.0873; $\mathbf{R}_{f} 0.54$ (hexane/EtOAc, 4/1). 


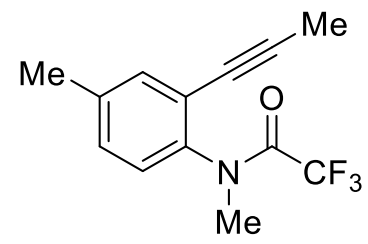

$1 c \quad$ (2,2,2-trifluoro- $N$-methyl- $N$-(4-methyl-2-(prop-1-yn-1-yl)phenyl)acetamide (from $5.0 \mathrm{mmol}$ of $\mathbf{S 2}$ to $1 \mathrm{c}, 1.10 \mathrm{~g}, 86 \%$ ): dark orange liquid; ${ }^{1} \mathbf{H}$ NMR (600 MHz, $\left.\mathbf{C D C l}_{3}\right) \delta 7.28(\mathrm{~d}, J=2.1 \mathrm{~Hz}, 1 \mathrm{H}), 7.11(\mathrm{dd}, J=8.0,2.1 \mathrm{~Hz}, 1 \mathrm{H}), 7.08(\mathrm{~d}$, $J=8.0 \mathrm{~Hz}, 1 \mathrm{H}), 3.31(\mathrm{~s}, 3 \mathrm{H}), 2.34$ (s, 3H), 2.03 (s, 3H); ${ }^{13} \mathbf{C}$ NMR (151 MHz, $\left.\mathbf{C D C l}_{3}\right) \delta 157.5\left(\mathrm{q},{ }^{2} J_{C-F}=36.1 \mathrm{~Hz}\right), 139.4,139.3,133.8,129.4,128.0\left(\mathrm{q},{ }^{4} J_{C-F}=1.5 \mathrm{~Hz}\right), 123.4,116.5(\mathrm{q}$, $J=288.2 \mathrm{~Hz}$ ), 91.8, 75.2, 38.5, 21.1, 4.6; IR (neat): $v_{\max }=2923,2237,1698,1499,1196,1149,739 \mathrm{~cm}^{-1}$; HRMS m/z (EI) calc. for $\mathrm{C}_{13} \mathrm{H}_{12} \mathrm{~F}_{3} \mathrm{NO}\left[\mathrm{M}^{+}\right]$255.0871, found 255.0868; $\mathbf{R}_{f} 0.57$ (hexane/EtOAc, 4/1).

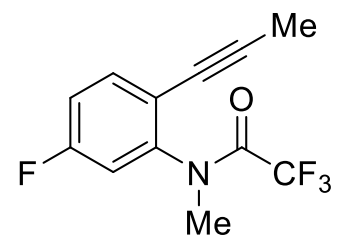

1 (2,2,2-trifluoro- $N$-(5-fluoro-2-(prop-1-yn-1-yl)phenyl)- $N$-methylacetamide (from $5.0 \mathrm{mmol}$ of $\mathbf{S 2}$ to $\mathbf{1 d}, 1.10 \mathrm{~g}, 85 \%$ ): pale yellow liquid; ${ }^{1} \mathbf{H}$ NMR (600 MHz, $\left.\mathbf{C D C l}_{3}\right) \delta 7.44\left(\mathrm{dd}, J=8.5,{ }^{4} J_{H-F}=6.0 \mathrm{~Hz}, 1 \mathrm{H}\right), 7.06\left(\mathrm{ddd}, J_{H-F}=8.5, J=\right.$ 8.1, $2.6 \mathrm{~Hz}, 1 \mathrm{H}), 6.96\left(\mathrm{dd}, J_{H-F}=8.7, \mathrm{~J}=2.6 \mathrm{~Hz}, 1 \mathrm{H}\right), 3.32(\mathrm{~s}, 3 \mathrm{H}), 2.02(\mathrm{~s}, 3 \mathrm{H})$;

${ }^{13}$ C NMR (151 MHz, CDCl $) \delta 161.7\left(\mathrm{~d},{ }^{1} J_{C-F}=251.8 \mathrm{~Hz}\right), 157.2\left(\mathrm{q},{ }^{2} J_{C-F}=36.3 \mathrm{~Hz}\right), 134.5(\mathrm{~d}, J=8.9$ $\mathrm{Hz}), 133.6(\mathrm{~d}, J=10.3 \mathrm{~Hz}), 120.1(\mathrm{~d}, J=3.9 \mathrm{~Hz}), 116.6(\mathrm{~d}, J=21.6 \mathrm{~Hz}), 116.3\left(\mathrm{q},{ }^{1} J_{C-F}=288.1 \mathrm{~Hz}\right)$, $116.0(\mathrm{dd}, J=23.3,1.5 \mathrm{~Hz}), 92.1\left(\mathrm{~d}, J=1.7 \mathrm{~Hz}\right.$ ), 74.0, 38.2, 4.5; IR (neat): $v_{\max }=3074,2922,2262$, 1701, 1495, 1206, 1178, 1151, 1089, $737 \mathrm{~cm}^{-1}$; HRMS m/z (EI) calc. for $\mathrm{C}_{12} \mathrm{H}_{9} \mathrm{~F}_{4} \mathrm{NO}\left[\mathrm{M}^{+}\right] 259.0620$, found $259.0620 ; \mathbf{R}_{f} 0.56$ (hexane/EtOAc, 4/1).

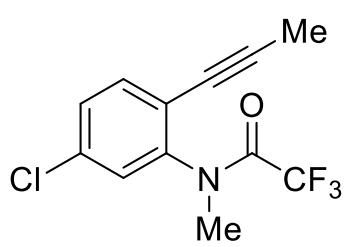

1 e $(N$-(5-chloro-2-(prop-1-yn-1-yl)phenyl)-2,2,2-trifluoro- $N$-methylacetamide (from $5.0 \mathrm{mmol}$ of $\mathbf{S 2}$ to $1 \mathbf{e}, 1.12 \mathrm{~g}, 81 \%$ ): orange liquid; ${ }^{1} \mathbf{H}$ NMR (600 MHz, $\left.\mathbf{C D C l}_{3}\right) \delta 7.38(\mathrm{~d}, J=8.3 \mathrm{~Hz}, 1 \mathrm{H}), 7.30(\mathrm{dd}, J=8.3,2.1 \mathrm{~Hz}, 1 \mathrm{H}), 7.23(\mathrm{~d}, J=2.1$ $\mathrm{Hz}, 1 \mathrm{H}), 3.30$ (s, 3H), 2.01 (s, 3H); ${ }^{13} \mathbf{C}$ NMR (151 MHz, CDCl$) \delta 157.1$ (q, $\left.{ }^{2} J_{C-F}=36.1 \mathrm{~Hz}\right), 142.8,134.0,133.8,129.5,128.6\left(\mathrm{q},{ }^{4} J_{C-F}=1.4 \mathrm{~Hz}\right), 122.4,116.3\left(\mathrm{q},{ }^{1} J_{C-F}=288.2 \mathrm{~Hz}\right)$, 93.5, 74.1, 38.2, 4.5; IR (neat): $v_{\max }=3070,2922,2233,1699,1485,1196,1150,826,730,515 \mathrm{~cm}^{-1}$; HRMS m/z (EI) calc. for $\mathrm{C}_{12} \mathrm{H}_{9} \mathrm{ClF}_{3} \mathrm{NO}\left[\mathrm{M}^{+}\right]$275.0325, found 275.0328; $\mathbf{R}_{f} 0.59$ (hexane/EtOAc, 4/1).

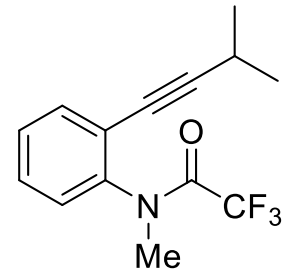

$1 f$ (2,2,2-trifluoro- $N$-methyl- $N$-(2-(3-methylbut-1-yn-1-yl)phenyl)acetamide (from $5.0 \mathrm{mmol}$ of $\mathbf{S 2}$ to $\mathbf{1 f}, 1.13 \mathrm{~g}, 84 \%)$ : orange liquid; ${ }^{1} \mathbf{H}$ NMR (600 $\left.\mathbf{M H z}, \mathbf{C D C l}_{3}\right) \delta$ $7.46-7.43(\mathrm{~m}, 1 \mathrm{H}), 7.32-7.29(\mathrm{~m}, 2 \mathrm{H}), 7.22-7.19(\mathrm{~m}, 1 \mathrm{H}), 3.32$ (s, 3H), 2.74 (hept, $J=6.8 \mathrm{~Hz}, 1 \mathrm{H}), 1.22(\mathrm{dd}, J=6.8,2.3 \mathrm{~Hz}, 6 \mathrm{H}) ;{ }^{13} \mathbf{C} \mathbf{~ N M R}\left(\mathbf{1 5 1} \mathbf{~ M H z}, \mathbf{C D C l}_{3}\right)$ $\delta 157.4\left(\mathrm{q},{ }^{2} J_{C-F}=36.0 \mathrm{~Hz}\right), 142.1,132.9,129.1,128.6,128.2\left(\mathrm{q},{ }^{4} J_{C-F}=1.1 \mathrm{~Hz}\right)$, $123.8,116.5\left(\mathrm{q},{ }^{1} J_{C-F}=288.2 \mathrm{~Hz}\right), 102.3,75.2,38.2,22.9,22.8,21.4$; IR (neat): $v_{\max }=2973,2935,2874$, 2232, 1697, 1489, 1196, 1151, $752 \mathrm{~cm}^{-1}$; HRMS m/z (EI) calc. for $\mathrm{C}_{14} \mathrm{H}_{14} \mathrm{~F}_{3} \mathrm{NO}\left[\mathrm{M}^{+}\right]$269.1027, found 269.1029; $\mathbf{R}_{f} 0.61$ (hexane/EtOAc, 4/1). 


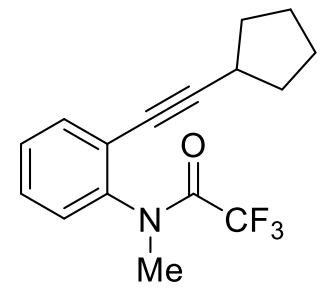

$1 g$ ( $N$-(2-(cyclopentylethynyl)phenyl)-2,2,2-trifluoro- $N$-methylacetamide (from 5.0 mmol of S2 to $1 \mathrm{~g}, 1.24 \mathrm{~g}, 84 \%)$ : yellow liquid; ${ }^{1} \mathbf{H}$ NMR (600 $\left.\mathbf{M H z}, \mathbf{C D C l}_{3}\right) \delta 7.42$ $(\mathrm{d}, J=6.9 \mathrm{~Hz}, 1 \mathrm{H}), 7.30-7.26(\mathrm{~m}, 2 \mathrm{H}), 7.19(\mathrm{~d}, J=6.9 \mathrm{~Hz}, 1 \mathrm{H}), 3.29(\mathrm{~s}, 3 \mathrm{H}), 2.79$ (p, $J=7.3 \mathrm{~Hz}, 1 \mathrm{H}), 1.95-1.90(\mathrm{~m}, 2 \mathrm{H}), 1.73-1.68(\mathrm{~m}, 2 \mathrm{H}), 1.66-1.61(\mathrm{~m}, 2 \mathrm{H})$, $1.58-1.53(\mathrm{~m}, 2 \mathrm{H}) ;{ }^{13} \mathbf{C}$ NMR (151 MHz, $\left.\mathbf{C D C l}_{3}\right) \delta 157.2\left(\mathrm{q},{ }^{2} J_{C-F}=35.8 \mathrm{~Hz}\right)$, 141.9, 132.8, 129.0, 128.4, 128.1, 123.9, $116.4\left(\mathrm{q},{ }^{1} J_{C-F}=288.2 \mathrm{~Hz}\right), 101.1,75.4,38.1,33.8,33.8,30.9$, 25.0, 25.0; IR (neat): $v_{\max }=2960,2872,2227,1697,1196,1150,752 \mathrm{~cm}^{-1}$; HRMS m/z (EI) calc. for $\mathrm{C}_{16} \mathrm{H}_{16} \mathrm{~F}_{3} \mathrm{NO}\left[\mathrm{M}^{+}\right]$295.1184, found 295.1183; $\mathbf{R}_{f} 0.60$ (hexane/EtOAc, 4/1).

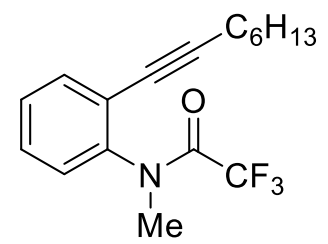

1 (2,2,2-trifluoro- $N$-methyl- $N$-(2-(oct-1-yn-1-yl)phenyl)acetamide (from 5.0 mmol of S2 to $1 \mathrm{~h}, 1.29 \mathrm{~g}, 83 \%)$ : orange liquid; ${ }^{1} \mathbf{H}$ NMR (600 $\left.\mathbf{M H z}, \mathbf{C D C l}_{3}\right) \delta$ $7.44(\mathrm{dd}, J=6.4,2.5 \mathrm{~Hz}, 1 \mathrm{H}), 7.30-7.27(\mathrm{~m}, 2 \mathrm{H}), 7.19$ (dd, $J=7.4,1.7 \mathrm{~Hz}, 1 \mathrm{H})$, 3.30 (s, 3H), 2.37 (t, $J=7.1 \mathrm{~Hz}, 2 \mathrm{H}), 1.54$ (tt, $J=7.3,7.1 \mathrm{~Hz}, 2 \mathrm{H}), 1.39$ (tt, $J=7.7$, $7.3 \mathrm{~Hz}, 2 \mathrm{H}), 1.31-1.26(\mathrm{~m}, 4 \mathrm{H}), 0.87(\mathrm{t}, J=7.0 \mathrm{~Hz}, 3 \mathrm{H}) ;{ }^{13} \mathbf{C} \mathbf{~ N M R}\left(\mathbf{1 5 1} \mathbf{M H z}, \mathbf{C D C l}_{3}\right) \delta 157.2\left(\mathrm{q},{ }^{2} J_{C-F}\right.$ $=35.9 \mathrm{~Hz}), 141.9,133.1,129.1,128.5,128.2(\mathrm{q}, J=1.4 \mathrm{~Hz}), 123.8,116.4\left(\mathrm{q},{ }^{1} J_{C-F}=288.2 \mathrm{~Hz}\right), 96.9$, 75.9, 38.2, 31.4, 28.6, 28.6, 22.6, 19.5, 14.1; IR (neat): $v_{\max }=2932,2860,2231,1700,1490,1217,1196$, 1151, 1123, $751 \mathrm{~cm}^{-1}$; HRMS m/z (EI) calc. for $\mathrm{C}_{17} \mathrm{H}_{20} \mathrm{~F}_{3} \mathrm{NO}\left[\mathrm{M}^{+}\right]$311.1497, found 311.1496; $\mathbf{R}_{f} 0.64$ (hexane/EtOAc, 4/1).

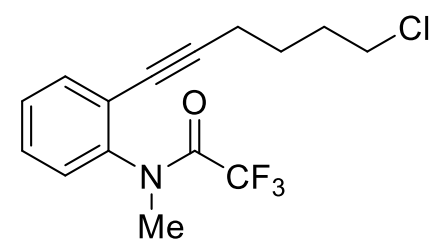

$1 i$ ( $N$-(2-(6-chlorohex-1-yn-1-yl)phenyl)-2,2,2-trifluoro- $N$-methylacetamide (from 5.0 mmol of $\mathbf{S 2}$ to $\mathbf{1 i}, 1.32 \mathrm{~g}, 83 \%$ ): pale yellow liquid; ${ }^{\mathbf{1}} \mathbf{H}$ NMR (600 MHz, $\left.\mathbf{C D C l}_{3}\right) \delta 77.39(\mathrm{dd}, J=5.9,3.3 \mathrm{~Hz}, 1 \mathrm{H}), 7.26-7.22(\mathrm{~m}, 2 \mathrm{H}), 7.15$ $(\mathrm{dd}, J=5.9,3.4 \mathrm{~Hz}, 1 \mathrm{H}), 3.48(\mathrm{t}, J=6.8 \mathrm{~Hz}, 2 \mathrm{H}), 3.23(\mathrm{~s}, 3 \mathrm{H}), 2.36(\mathrm{t}, J=$ $6.9 \mathrm{~Hz}, 2 \mathrm{H}), 1.81(\mathrm{tt}, J=7.4,6.8 \mathrm{~Hz}, 2 \mathrm{H}), 1.64(\mathrm{tt}, J=7.4,6.9 \mathrm{~Hz}, 2 \mathrm{H}) ;{ }^{13} \mathbf{C} \mathbf{N M R}\left(\mathbf{1 5 1} \mathbf{~ M H z}, \mathbf{C D C l}_{3}\right) \delta$ $156.7\left(\mathrm{q},{ }^{2} J_{C-F}=35.7 \mathrm{~Hz}\right), 141.7,132.8,128.9,128.6,128.1,128.1,123.3,116.2\left(\mathrm{q},{ }^{1} J_{C-F}=288.4 \mathrm{~Hz}\right)$, 95.5, 76.3, 44.3, 38.0, 31.3, 25.5, 18.5; IR (neat): $v_{\max }=2942,2870,2232,1698,1490,1196,1150,770$, $751 \mathrm{~cm}^{-1} ;$ HRMS m/z (EI) calc. for $\mathrm{C}_{15} \mathrm{H}_{15} \mathrm{ClF}_{3} \mathrm{NO}\left[\mathrm{M}^{+}\right]$317.0794, found 317.0792; $\mathbf{R}_{f} 0.53$ (hexane/EtOAc, 4/1).

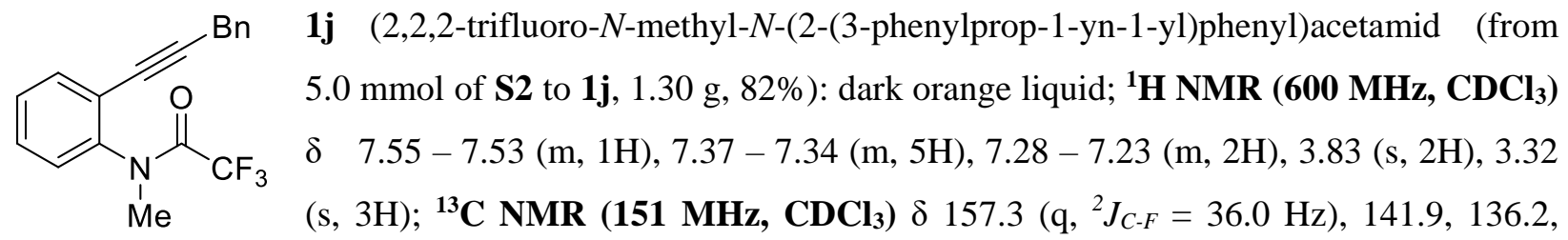
133.4, 129.2, 129.0, 128.9, 128.4, 128.0, 127.0, 123.4, 116.5 (q, $\left.{ }^{1} J_{C-F}=288.4 \mathrm{~Hz}\right), 94.1,78.0,38.4,26.0$; 
IR (neat): $v_{\max }=3065,3031,2926,2203,1698,1492,1199,1152,754 \mathrm{~cm}^{-1} ; \mathbf{H R M S ~ m / z ~ ( E I ) ~ c a l c . ~ f o r ~}$ $\mathrm{C}_{18} \mathrm{H}_{14} \mathrm{~F}_{3} \mathrm{NO}\left[\mathrm{M}^{+}\right]$317.1027, found 317.1028; $\mathbf{R}_{f} 0.50$ (hexane/EtOAc, 4/1).

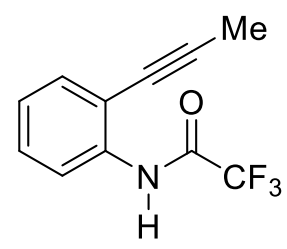

1k (2,2,2-trifluoro- $N$-(2-(prop-1-yn-1-yl)phenyl)acetamide ${ }^{\mathrm{S} 3}$ (from $10 \mathrm{mmol}$ of $\mathbf{S 1}$ to 1k, 1.77 g, 78\%): pale orange solid; ${ }^{1} \mathbf{H}$ NMR (600 $\left.\mathbf{M H z}, \mathbf{C D C l}_{3}\right) \delta 8.80(\mathrm{~s}, 1 \mathrm{H})$, $8.32(\mathrm{~d}, J=8.4 \mathrm{~Hz}, 1 \mathrm{H}), 7.42(\mathrm{dd}, J=7.8,1.5 \mathrm{~Hz}, 1 \mathrm{H}), 7.35(\mathrm{dd}, J=8.4,7.7 \mathrm{~Hz}, 1 \mathrm{H})$, 7.15 (ddd, $J=7.8,7.7,1.2 \mathrm{~Hz}, 1 \mathrm{H}), 2.16$ (s, 3H), 1.56 (s, 3H); ${ }^{13} \mathbf{C}$ NMR (151 MHz, $\left.\mathbf{C D C l}_{3}\right) \delta 154.6\left(\mathrm{q},{ }^{2} J_{C-F}=37.2 \mathrm{~Hz}\right), 136.4,131.9,129.3,125.6,121.1,119.8,119.6,115.9\left(\mathrm{q},{ }^{1} J_{C-F}=\right.$ $288.8 \mathrm{~Hz}$ ), 95.2, 74.2; IR (neat): $v_{\max }=3333,3075,2919,2854,2226,1711,1166,1145,1151,762 \mathrm{~cm}^{-1}$; $\mathbf{R}_{f} 0.24$ (hexane/EtOAc, 4/1).

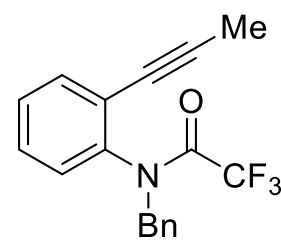

11 ( $N$-benzyl-2,2,2-trifluoro- $N$-(2-(prop-1-yn-1-yl)phenyl)acetamide (from $10 \mathrm{mmol}$ of $\mathbf{S 2}$ to 11, $2.73 \mathrm{~g}, 86 \%$ ): pale yellow liquid; ${ }^{1} \mathbf{H}$ NMR (600 $\left.\mathbf{~ M H z , ~} \mathbf{C D C l}_{3}\right) \delta 7.48(\mathrm{dd}$, $J=7.8,1.5 \mathrm{~Hz}, 1 \mathrm{H}), 7.30-7.26(\mathrm{~m}, 4 \mathrm{H}), 7.19-7.16(\mathrm{~m}, 2 \mathrm{H}), 7.12(\mathrm{ddd}, J=7.8,1.6$ $\mathrm{Hz}, 1 \mathrm{H}), 6.72(\mathrm{~d}, J=7.9 \mathrm{~Hz}, 1 \mathrm{H}), 5.59(\mathrm{~d}, J=14.1 \mathrm{~Hz}, 1 \mathrm{H}), 4.30(\mathrm{~d}, J=14.1 \mathrm{~Hz}, 1 \mathrm{H})$, $2.06(\mathrm{~s}, 3 \mathrm{H}) ;{ }^{13} \mathbf{C}$ NMR (151 MHz, $\left.\mathbf{C D C l}_{3}\right) \delta 157.4\left(\mathrm{q},{ }^{2} J_{C-F}=35.9 \mathrm{~Hz}\right), 139.8,135.5,133.2,130.0(\mathrm{q}, J=$ $1.4 \mathrm{~Hz}), 129.1,128.7,128.3,127.9,124.1,116.6$ (q, $\left.{ }^{1} J_{C-F}=288.6 \mathrm{~Hz}\right), 92.7,75.4,54.1,4.7$; IR (neat): $v_{\max }=3066,3033,2920,2239,1694,1489,1200,1174,1149,750,698 \mathrm{~cm}^{-1}$; HRMS m/z (EI) calc. for $\mathrm{C}_{18} \mathrm{H}_{14} \mathrm{~F}_{3} \mathrm{NO}\left[\mathrm{M}^{+}\right]$317.1027, found 317.1026; $\mathbf{R}_{f} 0.60$ (hexane/EtOAc, 4/1).

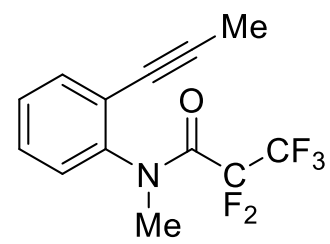

$1 \mathrm{~m}$ ( $N$-methyl- $N$-(2-(prop-1-yn-1-yl)phenyl)benzamide (from $5.0 \mathrm{mmol}$ of $\mathbf{S 4}$ to 1m, 1.24 g, 85\%): orange liquid; ${ }^{1} \mathbf{H}$ NMR (600 $\left.\mathbf{~ M H z , ~} \mathbf{C D C l}_{3}\right) \delta 7.47(\mathrm{dd}, J=4.6$ $\mathrm{Hz}, 1 \mathrm{H}), 7.34-7.31(\mathrm{~m}, 2 \mathrm{H}), 7.21$ (ddd, $J=4.6,1.6 \mathrm{~Hz}, 1 \mathrm{H}), 3.33(\mathrm{~s}, 3 \mathrm{H}), 2.04(\mathrm{~s}$, 3H); ${ }^{13}$ C NMR (151 MHz, CDCl $) \delta 158.1(\mathrm{t}, J=24.7 \mathrm{~Hz}), 142.1,133.1,129.0$, 128.7, $127.9(\mathrm{dd}, J=2.6,1.4 \mathrm{~Hz}), 123.6,118.3(\mathrm{qt}, J=286.7,34.3 \mathrm{~Hz}), 108.2(\mathrm{dd}, J=272.1,36.9 \mathrm{~Hz})$, 92.4, 75.0, 38.8, 4.5; IR (neat): $v_{\max }=2924,2236,1687,1491,1396,1224,1179,1128,1068,1036,966$, $747 \mathrm{~cm}^{-1}$; HRMS m/z (EI) calc. for $\mathrm{C}_{13} \mathrm{H}_{10} \mathrm{~F}_{5} \mathrm{NO}\left[\mathrm{M}^{+}\right]$291.0683, found 291.0685; $\mathbf{R}_{f} 0.59$ (hexane/EtOAc, 4/1).

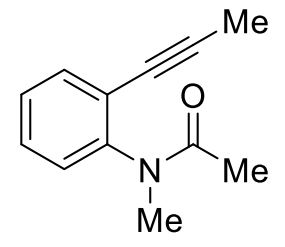

1n ( $N$-methyl- $N$-(2-(prop-1-yn-1-yl)phenyl)acetamide (from $10 \mathrm{mmol}$ of $\mathbf{S 4}$ to $\mathbf{1 n}$, $1.54 \mathrm{~g}, 78 \%)$ : orange liquid; ${ }^{1} \mathbf{H}$ NMR $\left(\mathbf{6 0 0} \mathbf{~ M H z}, \mathbf{C D C l}_{3}\right) \delta 7.46(\mathrm{dd}, J=7.5,1.7 \mathrm{~Hz}$, 1H), 7.31 (ddd, $J=7.8,7.6,1.7 \mathrm{~Hz}, 1 \mathrm{H}), 7.27$ (ddd, $J=7.6,7.5,1.4 \mathrm{~Hz}, 1 \mathrm{H}), 7.16$ (dd, $J=7.8,1.4 \mathrm{~Hz}, 1 \mathrm{H}), 3.22(\mathrm{~s}, 3 \mathrm{H}), 2.04(\mathrm{~s}, 3 \mathrm{H}), 1.83(\mathrm{~s}, 3 \mathrm{H}) ;{ }^{13} \mathbf{C}$ NMR (151 MHz, $\left.\mathbf{C D C l}_{3}\right) \delta 171.0,145.8,133.5,129.1,128.1,128.1,123.5,91.6,75.6,36.2,22.3,4.7$; IR (neat): $v_{\max }=$ 
2964, 2918, 2232, 1655, 1489, 1375, 1301, 1142, 753, $523 \mathrm{~cm}^{-1}$; HRMS m/z (EI) calc. for $\mathrm{C}_{12} \mathrm{H}_{13} \mathrm{NO}\left[\mathrm{M}^{+}\right.$] 187.0997, found 187.0995; $\mathbf{R}_{f} 0.14$ (hexane/EtOAc, 4/1).

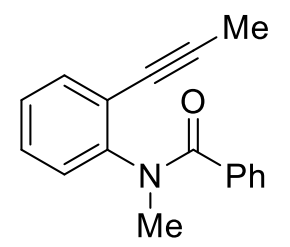

10 ( $N$-methyl- $N$-(2-(prop-1-yn-1-yl)phenyl)benzamide (from $5.0 \mathrm{mmol}$ of $\mathbf{S 4}$ to $\mathbf{1 0}$, 1.05 g, 84\%): dark orange liquid; ${ }^{1} \mathbf{H}$ NMR (600 $\left.\mathbf{M H z}, \mathbf{C D C l}_{3}\right) \delta 7.34-7.29$ (m, 3H), $7.17(\mathrm{t}, J=7.5 \mathrm{~Hz}, 1 \mathrm{H}), 7.13-7.05(\mathrm{~m}, 4 \mathrm{H}), 6.97-6.92(\mathrm{~m}, 1 \mathrm{H}), 3.42(\mathrm{~s}, 3 \mathrm{H}), 2.08(\mathrm{~s}$, $3 \mathrm{H}) ;{ }^{13} \mathbf{C}$ NMR (151 MHz, $\left.\mathbf{C D C l}_{3}\right) \delta 171.2,146.4,136.3,133.2,129.6,128.8,128.5$, 128.4, 127.6, 127.2, 122.9, 92.2, 76.3, 37.4, 4.7; IR (neat): $v_{\max }=3060,3028,2916,2232,1644,1489$, 1447, 1361, $715 \mathrm{~cm}^{-1}$; HRMS m/z (EI) calc. for $\mathrm{C}_{17} \mathrm{H}_{15} \mathrm{NO}\left[\mathrm{M}^{+}\right] 249.1154$, found 249.1153; $\mathbf{R}_{f} 0.20$ (hexane/EtOAc, 4/1).

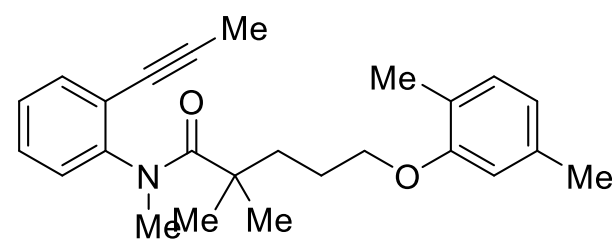

$1 p$ (5-(2,5-dimethylphenoxy)- $N, 2,2$-trimethyl- $N$-(2-(prop-1-yn1-yl)phenyl)pentanamide (from $1.0 \mathrm{mmol}$ of $\mathbf{S 4}$ to $\mathbf{1 p}, 321 \mathrm{mg}$, 85\%): orange liquid; ${ }^{1} \mathbf{H}$ NMR (600 $\left.\mathbf{M H z}, \mathbf{C D C l}_{3}\right) \delta 7.46-$ $7.44(\mathrm{~m}, 1 \mathrm{H}), 7.29-7.25(\mathrm{~m}, 2 \mathrm{H}), 7.19(\mathrm{dd}, J=7.0,2.2 \mathrm{~Hz}$, 1H), $7.00(\mathrm{~d}, J=7.6 \mathrm{~Hz}, 1 \mathrm{H}), 6.66(\mathrm{dd}, J=7.6,1.4 \mathrm{~Hz}, 1 \mathrm{H}), 6.63(\mathrm{~d}, J=1.4 \mathrm{~Hz}, 1 \mathrm{H}), 3.93$ (t, $J=5.5 \mathrm{~Hz}$, 2H), 3.23 (s, 3H), 2.32 (s, 3H), 2.18 (s, 3H), 2.03 (s, 3H), 1.96 - $1.72(\mathrm{~m}, 4 \mathrm{H}), 1.02(\mathrm{~s}, 6 \mathrm{H}) ;{ }^{13} \mathbf{C}$ NMR (151 MHz, $\left.\mathbf{C D C l}_{3}\right) \delta 176.9,157.2,146.7,136.6,133.2,130.4,129.1,128.4,128.0,123.6,120.7,112.1$, $76.4,68.4,44.4,39.9,39.6,27.6,26.9,25.6,21.6,16.0,4.5$.

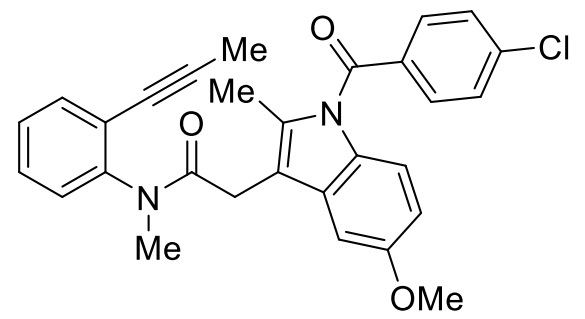

$1 q$ (2-(1-(4-chlorobenzoyl)-5-methoxy-2-methyl-1H-indol-3-yl)$N$-methyl- $N$-(2-(prop-1-yn-1-yl)phenyl)acetamide (from $1.0 \mathrm{mmol}$ of $\mathbf{S 4}$ to 1q, $407 \mathrm{mg}, 84 \%)$ : orange liquid; ${ }^{1} \mathbf{H}$ NMR (600 MHz, $\left.\mathbf{C D C l}_{3}\right) \delta 7.63(\mathrm{~d}, J=8.5 \mathrm{~Hz}, 2 \mathrm{H}), 7.47-7.43(\mathrm{~m}, 3 \mathrm{H}), 7.31(\mathrm{ddd}$, $J=7.6,7.5,1.7 \mathrm{~Hz}, 1 \mathrm{H}), 7.27(\mathrm{ddd}, J=7.7,7.5,1.5 \mathrm{~Hz}, 1 \mathrm{H}), 7.16$ $(\mathrm{dd}, J=7.7,1.5 \mathrm{~Hz}, 1 \mathrm{H}), 6.99(\mathrm{~d}, J=2.5 \mathrm{~Hz}, 1 \mathrm{H}), 6.79(\mathrm{~d}, J=8.9 \mathrm{~Hz}, 1 \mathrm{H}), 6.59$ (dd, $J=8.9,2.5 \mathrm{~Hz}, 1 \mathrm{H})$, $3.81(\mathrm{~s}, 3 \mathrm{H}), 3.53$ (d, $J=15.9 \mathrm{~Hz}, 1 \mathrm{H}), 3.45$ (d, $J=15.9 \mathrm{~Hz}, 1 \mathrm{H}), 3.24$ (s, 3H), 1.97 (s, 3H), 1.92 (s, 3H); ${ }^{13}$ C NMR (151 MHz, CDCl $) \delta 170.2,168.3,156.0,145.1,139.1,135.8,134.3,133.5,131.4,131.3$, 131.0, 129.1, 128.4, 128.3, 123.7, 114.8, 113.7, 111.3, 102.3, 92.1, 75.7, 55.8, 36.8, 31.0, 13.0, 4.4. 


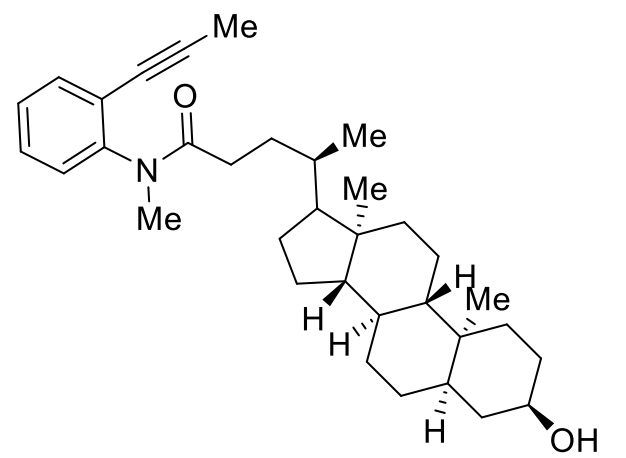

$1 \mathbf{2} \quad((4 \mathrm{R})-4-((5 \mathrm{~S}, 8 \mathrm{~S}, 9 \mathrm{R}, 10 \mathrm{R}, 13 \mathrm{~S}, 14 \mathrm{~S}, 15 \mathrm{R})-3$-hydroxy-10,14dimethylhexadecahydro- $1 H$-cyclopenta[a]phenanthren-15-yl)- $N$ methyl- $N$-(2-(prop-1-yn-1-yl)phenyl)pentanamide (from 1.0 mmol of S4 to 1r, $363 \mathrm{mg}, 72 \%$ ): dark orange liquid; ${ }^{1} \mathbf{H}$ NMR (600 MHz, $\left.\mathbf{C D C l}_{3}\right) \delta 7.44-7.41(\mathrm{~m}, 1 \mathrm{H}), 7.28-7.26(\mathrm{~m}, 2 \mathrm{H})$, $7.23-7.20(\mathrm{~m}, 1 \mathrm{H}), 4.53(\mathrm{~s}, 1 \mathrm{H}), 3.32(\mathrm{~s}, 3 \mathrm{H}), 2.48-2.40(\mathrm{~m}$, $1 \mathrm{H}), 2.37-2.30(\mathrm{~m}, 1 \mathrm{H}), 2.07(\mathrm{~s}, 3 \mathrm{H}), 2.01-0.93(\mathrm{~m}, 29 \mathrm{H})$, $0.91(\mathrm{~d}, J=6.5 \mathrm{~Hz}, 3 \mathrm{H}), 0.85(\mathrm{~d}, J=2.6 \mathrm{~Hz}, 3 \mathrm{H}), 0.62(\mathrm{~s}, 3 \mathrm{H}) ;{ }^{13} \mathbf{C}$ NMR (151 MHz, CDCl $) \delta 212.6$, 161.9, 161.6, 143.4, 133.3, 128.6, 128.5, 128.2, 92.5, 76.3, 56.8, 56.4, 42.9, 41.8, 40.5, 40.4, 39.9, 36.0, 35.9, 35.6, 35.0, 34.7, 30.1, 28.4, 27.1, 26.5, 24.4, 23.4, 23.4, 21.0, 18.7, 12.2, 4.8.

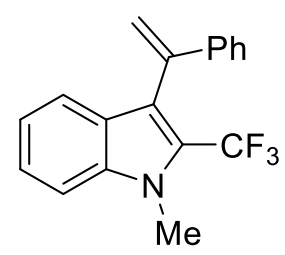

3aa (1-methyl-3-(1-phenylvinyl)-2-(trifluoromethyl)-1H-indole (137 mg, 91\%): pale yellow solid; m.p. 50-55 ${ }^{\circ} \mathrm{C}$; ${ }^{1} \mathbf{H}$ NMR (600 $\left.\mathbf{M H z}, \mathbf{C D C l}_{3}\right) \delta 7.54(\mathrm{~d}, J=8.1 \mathrm{~Hz}, 1 \mathrm{H})$, $7.46-7.41(\mathrm{~m}, 4 \mathrm{H}), 7.35-7.30(\mathrm{~m}, 3 \mathrm{H}), 7.19(\mathrm{ddd}, J=8.1,6.1,1.8 \mathrm{~Hz}, 1 \mathrm{H}), 6.05(\mathrm{~s}$, 1H), $5.45(\mathrm{~s}, 1 \mathrm{H}), 3.94(\mathrm{~s}, 3 \mathrm{H}) ;{ }^{13} \mathbf{C}$ NMR (151 MHz, $\left.\mathbf{C D C l}_{3}\right) \delta 141.0\left(\mathrm{q},{ }^{4} \mathrm{~J}_{C-F}=1.2\right.$ $\mathrm{Hz}), 140.2,137.8,128.5,127.9,127.0,126.5,125.1,123.5\left(\mathrm{q},{ }^{2} J_{C-F}=35.1 \mathrm{~Hz}\right), 122.0\left(\mathrm{q},{ }^{1} J_{C-F}=270.2\right.$ $\mathrm{Hz}), 121.7,120.9,119.8\left(\mathrm{q},{ }^{3} J_{C-F}=3.0 \mathrm{~Hz}\right), 117.6\left(\mathrm{q},{ }^{4} J_{C-F}=1.6 \mathrm{~Hz}\right), 109.8,31.3\left(\mathrm{q},{ }^{4} J_{C-F}=2.6 \mathrm{~Hz}\right) ; \mathbf{I R}$ (neat): $v_{\max }=3059,3030,2953,1469,1420,1253,1230,1172,1158,1103,1076,745 \mathrm{~cm}^{-1} ; \mathbf{H R M S ~ m} / \mathrm{z}$ (EI) calc. for $\mathrm{C}_{18} \mathrm{H}_{14} \mathrm{~F}_{3} \mathrm{~N}\left[\mathrm{M}^{+}\right]$301.1078, found 301.1078; $\mathbf{R}_{f} 0.73$ (hexane/EtOAc, 4/1).

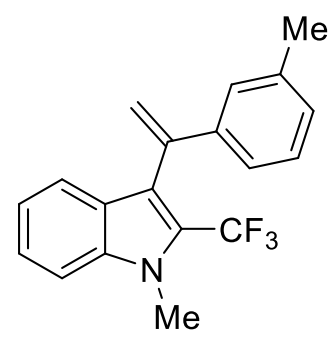

$3 \mathbf{a b}$ (1-methyl-3-(1-(m-tolyl)vinyl)-2-(trifluoromethyl)-1H-indole (140 mg, 89\%): orange liquid; ${ }^{1} \mathbf{H}$ NMR $\left(600 ~ \mathbf{M H z}, \mathbf{C D C l}_{3}\right) \delta 7.65(\mathrm{~d}, J=8.0 \mathrm{~Hz}, 1 \mathrm{H}), 7.53-7.48$ (m, 2H), 7.37 (s, 1H), $7.32-7.25$ (m, 3H), 7.21 (dd, $J=5.2,3.4 \mathrm{~Hz}, 1 \mathrm{H}), 6.13$ (d, $J=1.4 \mathrm{~Hz}, 1 \mathrm{H}), 5.51(\mathrm{~d}, J=1.4 \mathrm{~Hz}, 1 \mathrm{H}), 3.99(\mathrm{~s}, 3 \mathrm{H}), 2.44(\mathrm{~s}, 3 \mathrm{H}) ;{ }^{13} \mathbf{C} \mathbf{~ N M R}$ (151 MHz, CDCl $\mathbf{l}_{3} \delta 141.0(\mathrm{q}, \mathrm{J}=1.1 \mathrm{~Hz}), 140.4,138.0,137.8,128.7,128.4$, $127.1,127.1,125.1,123.3\left(\mathrm{q},{ }^{2} J_{C-F}=35.1 \mathrm{~Hz}\right), 122.1\left(\mathrm{q},{ }^{1} J_{C-F}=270.2 \mathrm{~Hz}\right), 121.7$, $120.9,120.0\left(\mathrm{q},{ }^{3} J_{C-F}=3.0 \mathrm{~Hz}\right), 117.5\left(\mathrm{q},{ }^{4} J_{C-F}=1.5 \mathrm{~Hz}\right), 109.8,31.2\left(\mathrm{q},{ }^{4} J_{C-F}=2.7 \mathrm{~Hz}\right), 21.6$; IR (neat): $v_{\max }=3056,2924,2854,1469,1252,1157,1101,1074,745 \mathrm{~cm}^{-1} ; \mathbf{H R M S ~ m / z ~ ( E I ) ~ c a l c . ~ f o r ~} \mathrm{C}_{19} \mathrm{H}_{16} \mathrm{~F}_{3} \mathrm{~N}$ $\left[\mathrm{M}^{+}\right]$315.1235, found 315.1236; $\mathbf{R}_{f} 0.76$ (hexane/EtOAc, 4/1). 


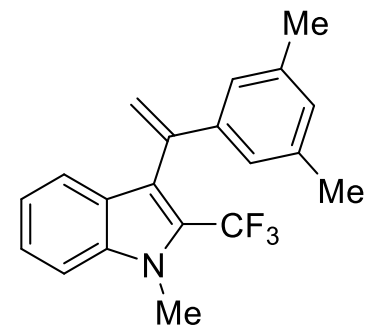

$120.9,120.1\left(\mathrm{q},{ }^{3} J_{C-F}=3.2 \mathrm{~Hz}\right), 117.4,117.3,109.8,31.2\left(\mathrm{q},{ }^{4} J_{C-F}=2.8 \mathrm{~Hz}\right), 21.5$; IR (neat): $v_{\max }=2919$, 2862, 1468, 1416, 1257, 1211, 1157, 1101, 1076, $743 \mathrm{~cm}^{-1} ; \mathbf{H R M S ~ m / z ~ ( E I ) ~ c a l c . ~ f o r ~} \mathrm{C}_{20} \mathrm{H}_{18} \mathrm{~F}_{3} \mathrm{~N}\left[\mathrm{M}^{+}\right]$ 329.1391, found 329.1389; $\mathbf{R}_{f} 0.76$ (hexane/EtOAc, 4/1).

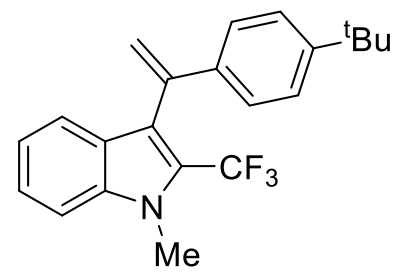

3ad (3-(1-(4-(tert-butyl)phenyl)vinyl)-1-methyl-2-(trifluoromethyl)- $1 H$-indole (148 mg, 83\%): yellow liquid; ${ }^{1} \mathbf{H}$ NMR (600 MHz, $\left.\mathbf{C D C l}_{3}\right) \delta 7.54(\mathrm{~d}, J=8.1$ $\mathrm{Hz}, 1 \mathrm{H}), 7.45(\mathrm{~d}, J=8.2 \mathrm{~Hz}, 1 \mathrm{H}), 7.42(\mathrm{dd}, J=8.2,6.5 \mathrm{~Hz}, 1 \mathrm{H}), 7.38-7.35$ (m, 4H), 7.18 (ddd, $J=8.1,6.5,1.7 \mathrm{~Hz}, 1 \mathrm{H}), 6.04(\mathrm{~d}, J=1.5 \mathrm{~Hz}, 1 \mathrm{H}), 5.39$ (d, $J=1.5 \mathrm{~Hz}, 1 \mathrm{H}), 3.95$ (s, 3H), 1.37 (s, 9H); ${ }^{13} \mathbf{C}$ NMR (151 MHz, CDCl $) \delta$ $150.9,139.9,137.9,137.8,137.8,127.1,126.2,125.4,125.0,123.5\left(\mathrm{q},{ }^{2} J_{C-F}=35.0 \mathrm{~Hz}\right), 122.1\left(\mathrm{q},{ }^{1} J_{C-F}=\right.$ $270.2 \mathrm{~Hz}), 121.8,120.8,120.0\left(\mathrm{q},{ }^{3} J_{C-F}=3.1 \mathrm{~Hz}\right), 116.8\left(\mathrm{q},{ }^{4} J_{C-F}=1.6 \mathrm{~Hz}\right), 109.8,34.7,31.5,31.3\left(\mathrm{q},{ }^{4} J_{C-}\right.$ ${ }_{F}=2.6 \mathrm{~Hz}$ ); IR (neat): $v_{\max }=3053,2960,2867,1468,1252,1172,1157,1103,1073,743 \mathrm{~cm}^{-1} ; \mathbf{H R M S}$ $\mathrm{m} / \mathrm{z}$ (EI) calc. for $\mathrm{C}_{22} \mathrm{H}_{22} \mathrm{~F}_{3} \mathrm{~N}\left[\mathrm{M}^{+}\right] 357.1704$, found 357.1705; $\mathbf{R}_{f} 0.76$ (hexane/EtOAc, 4/1).

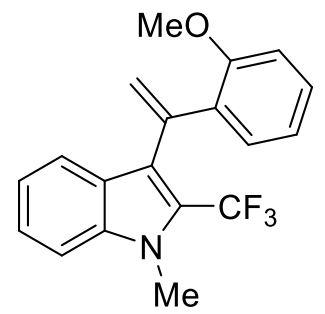

3ae (3-(1-(2-methoxyphenyl)vinyl)-1-methyl-2-(trifluoromethyl)-1H-indole (137 mg, 83\%): orange liquid; ${ }^{1} \mathbf{H}$ NMR $\left(\mathbf{6 0 0} \mathbf{~ M H z}, \mathbf{C D C l}_{3}\right) \delta 7.55(\mathrm{~d}, J=8.1 \mathrm{~Hz}, 1 \mathrm{H})$, $7.39-7.35$ (m, 2H), $7.27-7.23(\mathrm{~m}, 2 \mathrm{H}), 7.13(\mathrm{ddd}, J=7.9,6.0,1.9 \mathrm{~Hz}, 1 \mathrm{H}), 6.92$ - $6.89(\mathrm{~m}, 2 \mathrm{H}), 6.01(\mathrm{~d}, J=2.1 \mathrm{~Hz}, 1 \mathrm{H}), 5.56(\mathrm{~d}, J=2.1 \mathrm{~Hz}, 1 \mathrm{H}), 3.89(\mathrm{~s}, 2 \mathrm{H}), 3.71$ $(\mathrm{s}, 3 \mathrm{H}) ;{ }^{13} \mathbf{C}$ NMR (151 MHz, $\left.\mathbf{C D C l}_{3}\right) \delta$ 157.4, 137.6, 137.5, 130.9, 130.5, 128.8, 126.9, 124.7, $122.7\left(\mathrm{q},{ }^{2} J_{C-F}=35.1 \mathrm{~Hz}\right), 122.2\left(\mathrm{q},{ }^{1} J_{C-F}=270.1 \mathrm{~Hz}\right), 121.9,121.7\left(\mathrm{q},{ }^{3} J_{C-F}=2.8 \mathrm{~Hz}\right)$, $121.6\left(\mathrm{q},{ }^{4} J_{C-F}=1.7 \mathrm{~Hz}\right), 120.6,120.6,111.7,109.6,55.6,31.2\left(\mathrm{q},{ }^{4} J_{C-F}=2.6 \mathrm{~Hz}\right) ;$ IR (neat): $v_{\max }=3060$, 2930, 2836, 1466, 1251, 1226, 1170, 1157, 1097, 1073, 1027, $743 \mathrm{~cm}^{-1}$; HRMS m/z (EI) calc. for $\mathrm{C}_{19} \mathrm{H}_{16} \mathrm{~F}_{3} \mathrm{NO}\left[\mathrm{M}^{+}\right]$331.1184, found 331.1180; $\mathbf{R}_{f} 0.69$ (hexane/EtOAc, 4/1).

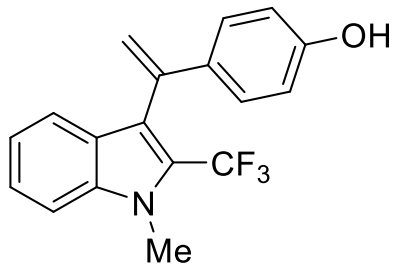

3af (4-(1-(1-methyl-2-(trifluoromethyl)-1H-indol-3-yl)vinyl)phenol (128 mg, 81\%): dark orange liquid; ${ }^{1} \mathbf{H}$ NMR $\left(600 \mathbf{~ M H z}, \mathbf{C D C l}_{3}\right) \delta 7.48(\mathrm{~d}, J=8.0 \mathrm{~Hz}$, $1 \mathrm{H}), 7.42-7.37(\mathrm{~m}, 2 \mathrm{H}), 7.25(\mathrm{~d}, J=8.8 \mathrm{~Hz}, 2 \mathrm{H}), 7.14$ (ddd, $J=8.0,6.5,1.5$ $\mathrm{Hz}, 1 \mathrm{H}), 6.74(\mathrm{~d}, J=8.8 \mathrm{~Hz}, 2 \mathrm{H}), 5.89(\mathrm{~d}, J=1.3 \mathrm{~Hz}, 1 \mathrm{H}), 5.28(\mathrm{~d}, J=1.3$ 
Hz, 1H), 4.98 (s, 1H), 3.91 (s, 3H); ${ }^{13} \mathbf{C}$ NMR (151 MHz, CDCl $) \delta$ 155.4, 139.4, 137.8, 133.9, 128.0, 127.0, 125.0, $123.4\left(\mathrm{q},{ }^{2} J_{C-F}=35.2 \mathrm{~Hz}\right), 122.0\left(\mathrm{q},{ }^{1} J_{C-F}=270.2 \mathrm{~Hz}\right), 121.7,120.9,120.0\left(\mathrm{q},{ }^{3} J_{C-F}=2.9\right.$ $\mathrm{Hz}), 115.8\left(\mathrm{q},{ }^{4} J_{C-F}=1.5 \mathrm{~Hz}\right), 115.3,109.8,31.3\left(\mathrm{q},{ }^{4} J_{C-F}=2.6 \mathrm{~Hz}\right) ; \mathbf{I R}$ (neat): $v_{\max }=3367,3058,2929$, 2851, 1736, 1512, 1373, 1251, 1233, 1173, 1160, 1108, 1044, $746 \mathrm{~cm}^{-1}$; HRMS m/z (EI) calc. for $\mathrm{C}_{18} \mathrm{H}_{14} \mathrm{~F}_{3} \mathrm{NO}\left[\mathrm{M}^{+}\right]$317.1027, found 317.1027; $\mathbf{R}_{f} 0.26$ (hexane/EtOAc, 4/1).

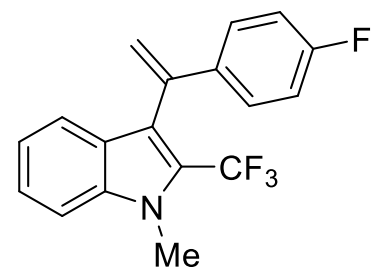

$3 a g$ (3-(1-(4-fluorophenyl)vinyl)-1-methyl-2-(trifluoromethyl)-1H-indole (142 mg, 89\%): orange liquid; ${ }^{1} \mathbf{H}$ NMR $\left(600 \mathbf{~ M H z}, \mathbf{C D C l}_{3}\right) \delta 7.54(\mathrm{~d}, J=8.0 \mathrm{~Hz}$, $1 \mathrm{H}), 7.47-7.43(\mathrm{~m}, 2 \mathrm{H}), 7.39\left(\mathrm{dd}, J=8.8,{ }^{3} J_{H-F}=5.4 \mathrm{~Hz}, 2 \mathrm{H}\right), 7.20$ (ddd, $J=$ $8.0,5.9,2.0 \mathrm{~Hz}, 1 \mathrm{H}), 7.02\left(\mathrm{dd}, \mathrm{z} J_{H-F}=9.0, J=8.8 \mathrm{~Hz}, 2 \mathrm{H}\right), 5.98(\mathrm{~d}, J=1.2 \mathrm{~Hz}$, 1H), $5.43(\mathrm{~d}, J=1.2 \mathrm{~Hz}, 1 \mathrm{H}), 3.95(\mathrm{~s}, 3 \mathrm{H}) ;{ }^{13} \mathbf{C ~ N M R}\left(151 \mathrm{MHz}, \mathbf{C D C l}_{3}\right) \delta$ $162.7\left(\mathrm{~d},{ }^{1} J_{C-F}=246.8 \mathrm{~Hz}\right), 139.3,137.2(\mathrm{q}, J=1.4 \mathrm{~Hz}), 128.2\left(\mathrm{~d},{ }^{3} J_{C-F}=8.0 \mathrm{~Hz}\right), 126.9,125.2,123.5(\mathrm{q}$, $\left.{ }^{2} J_{C-F}=35.2 \mathrm{~Hz}\right), 122.0\left(\mathrm{q},{ }^{1} J_{C-F}=270.2 \mathrm{~Hz}\right), 121.5,121.0,119.6(\mathrm{q}, J=3.1 \mathrm{~Hz}), 117.4,117.4\left(\mathrm{~d},{ }^{4} J_{C-F}=\right.$ $2.7 \mathrm{~Hz}), 115.3\left(\mathrm{~d},{ }^{2} J_{C-F}=21.5 \mathrm{~Hz}\right), 109.9,31.3(\mathrm{q}, J=2.7 \mathrm{~Hz}) ; \mathbf{I R}\left(\right.$ neat): $v_{\max }=3054,2957,1507,1253$, 1231, 1173, 1158, 1110, 1075, $745 \mathrm{~cm}^{-1}$; HRMS m/z (EI) calc. for $\mathrm{C}_{18} \mathrm{H}_{13} \mathrm{~F}_{4} \mathrm{~N}$ [M+] 319.0984, found 319.0986; $\mathbf{R}_{f} 0.71$ (hexane/EtOAc, 4/1).

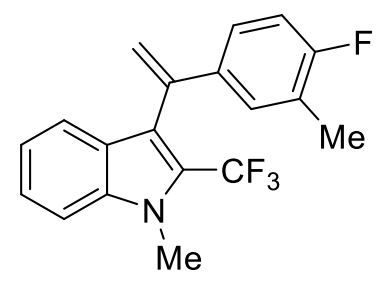

3ah (3-(1-(4-fluoro-3-methylphenyl)vinyl)-1-methyl-2-(trifluoromethyl)-1 Hindole (140 mg, 84\%): orange liquid; ${ }^{1} \mathbf{H}$ NMR (600 $\left.\mathbf{~ M H z , ~} \mathbf{C D C l}_{3}\right) \delta 7.53(\mathrm{~d}, J$ $=8.4 \mathrm{~Hz}, 1 \mathrm{H}), 7.46-7.41(\mathrm{~m}, 2 \mathrm{H}), 7.25\left(\mathrm{dd}, J=7.4,{ }^{4} J_{H-F}=2.7 \mathrm{~Hz}, 1 \mathrm{H}\right), 7.21$ $-7.16(\mathrm{~m}, 2 \mathrm{H}), 6.94(\mathrm{dd}, J=8.9,8.4 \mathrm{~Hz}, 1 \mathrm{H}), 5.95(\mathrm{~d}, J=1.3 \mathrm{~Hz}, 1 \mathrm{H}), 5.38(\mathrm{~d}$, $J=1.2 \mathrm{~Hz}, 1 \mathrm{H}), 3.94(\mathrm{~s}, 3 \mathrm{H}), 2.27\left(\mathrm{~d},{ }^{4} J_{H-F}=2.0 \mathrm{~Hz}, 3 \mathrm{H}\right) ;{ }^{13} \mathbf{C} \mathbf{~ N M R}(\mathbf{1 5 1} \mathbf{M H z}$, $\left.\mathbf{C D C l}_{3}\right) \delta 161.3\left(\mathrm{~d},{ }^{1} J_{C-F}=245.6 \mathrm{~Hz}\right), 139.4,137.8,136.9\left(\mathrm{~d},{ }^{4} J_{C-F}=3.1 \mathrm{~Hz}\right), 129.5\left(\mathrm{~d},{ }^{3} J_{C-F}=5.1 \mathrm{~Hz}\right)$, $126.9,125.6\left(\mathrm{~d},{ }^{3} J_{C-F}=8.0 \mathrm{~Hz}\right), 125.1,124.7\left(\mathrm{~d},{ }^{2} J_{C-F}=17.6 \mathrm{~Hz}\right), 123.5\left(\mathrm{q},{ }^{2} J_{C-F}=35.2 \mathrm{~Hz}\right), 122.0\left(\mathrm{q},{ }^{1} J_{C-}\right.$ $\left.{ }_{F}=270.2 \mathrm{~Hz}\right), 121.6,121.0,119.7\left(\mathrm{q},{ }^{3} J_{C-F}=2.9 \mathrm{~Hz}\right), 114.9\left(\mathrm{~d},{ }^{2} J_{C-F}=22.5 \mathrm{~Hz}\right), 109.9,31.3\left(\mathrm{q},{ }^{4} J_{C-F}=2.7\right.$ $\mathrm{Hz}), 14.8\left(\mathrm{~d},{ }^{3} J_{C-F}=3.5 \mathrm{~Hz}\right.$ ); IR (neat): $v_{\max }=3055,2927,2855,1501,1253,1244,1186,1161,1108$, 1076, $745 \mathrm{~cm}^{-1}$; HRMS m/z (EI) calc. for $\mathrm{C}_{19} \mathrm{H}_{15} \mathrm{~F}_{4} \mathrm{~N}\left[\mathrm{M}^{+}\right]$333.1141, found 333.1143; $\mathbf{R}_{f} 0.76$ (hexane/EtOAc, 4/1).

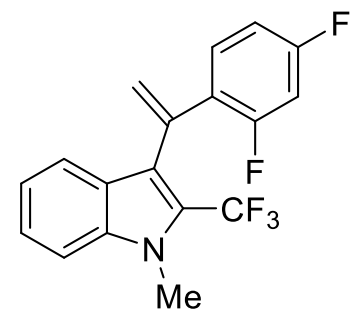

$3 a$ (3-(1-(2,4-difluorophenyl)vinyl)-1-methyl-2-(trifluoromethyl)-1H-indole (152 mg, 90\%): white solid, m.p. 65-70 ${ }^{\circ} \mathrm{C} ;{ }^{1} \mathbf{H}$ NMR (600 MHz, $\left.\mathbf{C D C l}_{3}\right) \delta 7.59$ $(\mathrm{d}, J=8.4 \mathrm{~Hz}, 1 \mathrm{H}), 7.45-7.43(\mathrm{~m}, 2 \mathrm{H}), 7.22\left(\mathrm{ddd}, J=7.9,{ }^{4} J_{H-F}=3.9,3.9 \mathrm{~Hz}\right.$, $1 \mathrm{H}), 7.18(\mathrm{ddd}, J=8.4,6.5 \mathrm{~Hz}, 1 \mathrm{H}), 6.86\left(\mathrm{ddd}, J_{H-F}=11.3,8.9, J=2.6 \mathrm{~Hz}, 1 \mathrm{H}\right)$, $6.80\left(\mathrm{dddd}, J_{H-F}=9.0, J=8.8,2.6,{ }^{5} J_{H-F}=1.0 \mathrm{~Hz}, 1 \mathrm{H}\right), 6.05(\mathrm{~s}, 1 \mathrm{H}), 5.68(\mathrm{~s}, 1 \mathrm{H})$, 
$3.92(\mathrm{~s}, 3 \mathrm{H}) ;{ }^{13} \mathbf{C}$ NMR $\left(151 \mathrm{MHz}, \mathbf{C D C l}_{3}\right) \delta 162.3\left(\mathrm{dd},{ }^{1} J_{C-F}=249.3,{ }^{3} J_{C-F}=11.9 \mathrm{~Hz}\right), 160.5\left(\mathrm{dd},{ }^{1} J_{C-F}=\right.$ $\left.252.8,{ }^{3} J_{C-F}=11.8 \mathrm{~Hz}\right), 137.7\left(\mathrm{~d},{ }^{4} J_{C-F}=1.4 \mathrm{~Hz}\right), 133.9,131.3\left(\mathrm{q},{ }^{3} J_{C-F}=4.6 \mathrm{~Hz}\right), 126.7,125.6\left(\mathrm{ddd},{ }^{2} J_{C-F}\right.$ $\left.=13.0,{ }^{4} J_{C-F}=3.5 \mathrm{~Hz}\right), 125.2,123.2\left(\mathrm{q},{ }^{2} J_{C-F}=35.3 \mathrm{~Hz}\right), 122.4\left(\mathrm{~d},{ }^{3} J_{C-F}=6.6 \mathrm{~Hz}\right), 122.0\left(\mathrm{q},{ }^{1} J_{C-F}=270.2\right.$ $\mathrm{Hz}), 121.3,121.1,120.0\left(\mathrm{q},{ }^{4} J_{C-F}=3.0 \mathrm{~Hz}\right), 111.2\left(\mathrm{dd},{ }^{2} J_{C-F}=21.0,{ }^{4} J_{C-F}=3.8 \mathrm{~Hz}\right), 109.9,104.5\left(\mathrm{dd},{ }^{2} J_{C-F}\right.$ $\left.=26.9,{ }^{2} J_{C-F}=25.3 \mathrm{~Hz}\right), 31.2\left(\mathrm{q},{ }^{4} J_{C-F}=2.5 \mathrm{~Hz}\right)$; IR (neat): $v_{\max }=3063,1502,1251,1171,1159,1102$, 1083, 1071, 968, $743 \mathrm{~cm}^{-1}$; HRMS m/z (EI) calc. for $\mathrm{C}_{18} \mathrm{H}_{12} \mathrm{~F}_{5} \mathrm{~N}\left[\mathrm{M}^{+}\right]$337.0890, found 337.0887; $\mathbf{R}_{f} 0.69$ (hexane/EtOAc, 4/1).

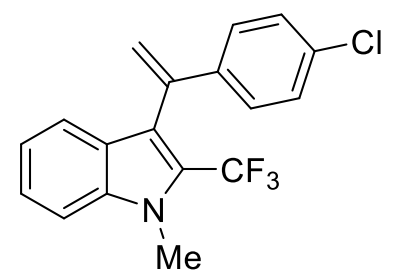

3aj (3-(1-(4-chlorophenyl)vinyl)-1-methyl-2-(trifluoromethyl)-1H-indole (143 mg, 85\%): yellow liquid; ${ }^{1} \mathbf{H}$ NMR $\left(600 \mathbf{~ M H z}, \mathbf{C D C l}_{3}\right) \delta 7.54(\mathrm{~d}, J=8.0 \mathrm{~Hz}$, 1H), $7.46-7.44(\mathrm{~m}, 3 \mathrm{H}), 7.30-7.28(\mathrm{~m}, 2 \mathrm{H}), 7.25(\mathrm{dd}, J=7.8,7.7 \mathrm{~Hz}, 1 \mathrm{H})$, $7.21(\mathrm{ddd}, J=8.0,5.7,2.2 \mathrm{~Hz}, 1 \mathrm{H}), 6.06(\mathrm{~d}, J=1.1 \mathrm{~Hz}, 1 \mathrm{H}), 5.49(\mathrm{~d}, J=1.1$ $\mathrm{Hz}, 1 \mathrm{H}), 3.95(\mathrm{~s}, 3 \mathrm{H}) ;{ }^{13} \mathbf{C}$ NMR $\left(\mathbf{1 5 1} \mathbf{~ M H z}, \mathbf{C D C l}_{3}\right) \delta 143.0\left(\mathrm{~d},{ }^{4} J_{C-F}=1.3 \mathrm{~Hz}\right)$, $139.2,137.8,134.5,129.7,127.9,126.8,126.6,125.2,124.8,123.6\left(\mathrm{q},{ }^{2} J_{C-F}=35.3 \mathrm{~Hz}\right), 121.9\left(\mathrm{q},{ }^{1} J_{C-F}=\right.$ $270.4 \mathrm{~Hz}), 121.4,121.1,119.0\left(\mathrm{q},{ }^{3} J_{C-F}=3.2 \mathrm{~Hz}\right), 118.9\left(\mathrm{q},{ }^{1} J_{C-F}=1.5 \mathrm{~Hz}\right), 109.9,31.3\left(\mathrm{q},{ }^{4} J_{C-F}=2.6 \mathrm{~Hz}\right)$; IR (neat): $v_{\max }=3062,2927,2852,1469,1251,1228,1173,1158,1104,1075,790,744 \mathrm{~cm}^{-1}$; HRMS $\mathrm{m} / \mathrm{z}$ (EI) calc. for $\mathrm{C}_{18} \mathrm{H}_{13} \mathrm{ClF}_{3} \mathrm{~N}\left[\mathrm{M}^{+}\right]$335.0689, found 335.0690; $\mathbf{R}_{f} 0.69$ (hexane/EtOAc, 4/1).

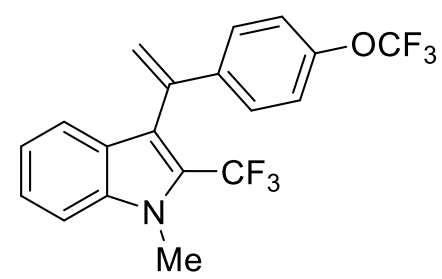

3ak (1-methyl-3-(1-(4-(trifluoromethoxy)phenyl)vinyl)-2(trifluoromethyl)-1H-indole (156 mg, 81\%): pale orange liquid; ${ }^{1} \mathbf{H}$ NMR $\left(600 \mathrm{MHz}, \mathbf{C D C l}_{3}\right) \delta 7.56(\mathrm{~d}, J=7.9 \mathrm{~Hz}, 1 \mathrm{H}), 7.49-7.45(\mathrm{~m}, 4 \mathrm{H}), 7.23$ (ddd, $J=8.4,6.1,1.9 \mathrm{~Hz}, 1 \mathrm{H}), 7.20(\mathrm{dd}, J=8.4,0.8 \mathrm{~Hz}, 2 \mathrm{H}), 6.05(\mathrm{~d}, J=$ $1.1 \mathrm{~Hz}, 1 \mathrm{H}), 5.50(\mathrm{~d}, J=1.1 \mathrm{~Hz}, 1 \mathrm{H}), 3.97(\mathrm{~s}, 3 \mathrm{H}) ;{ }^{13} \mathrm{C}$ NMR (151 MHz, $\left.\mathbf{C D C l}_{3}\right) \delta 149.0\left(\mathrm{q},{ }^{4} J_{C-F}=1.6 \mathrm{~Hz}\right), 139.8,139.1,137.9,128.0,126.9,125.3,123.6\left(\mathrm{q},{ }^{2} J_{C-F}=35.3 \mathrm{~Hz}\right)$, $122.0\left(\mathrm{q},{ }^{1} J_{C-F}=270.2 \mathrm{~Hz}\right), 121.5,121.2,120.9,120.8\left(\mathrm{q},{ }^{1} J_{C-F}=257.1 \mathrm{~Hz}\right), 119.3\left(\mathrm{q},{ }^{3} J_{C-F}=2.8 \mathrm{~Hz}\right)$, $118.4\left(\mathrm{q},{ }^{4} J_{C-F}=1.6 \mathrm{~Hz}\right), 110.0,31.2\left(\mathrm{q},{ }^{4} J_{C-F}=2.6 \mathrm{~Hz}\right) ; \mathbf{I R}\left(\right.$ neat) $: v_{\max }=3058,1506,1250,1222,1156$, 1106, 1074, $744 \mathrm{~cm}^{-1}$; HRMS m/z (EI) calc. for $\mathrm{C}_{19} \mathrm{H}_{13} \mathrm{~F}_{6} \mathrm{NO}\left[\mathrm{M}^{+}\right]$385.0901, found 385.0904; $\mathbf{R}_{f} 0.71$ (hexane/EtOAc, 4/1).

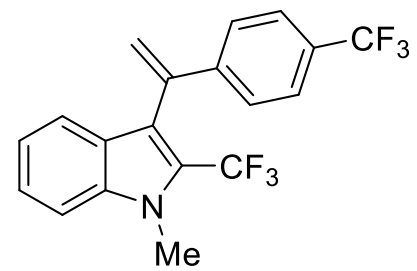

3al (1-methyl-2-(trifluoromethyl)-3-(1-(4-(trifluoromethyl)phenyl)vinyl)-1Hindole (151 mg, 82\%): yellow liquid; ${ }^{1} \mathbf{H}$ NMR (600 $\left.\mathbf{~ M H z , ~} \mathbf{C D C l}_{3}\right) \delta 7.60(\mathrm{~d}$, $J=8.2 \mathrm{~Hz}, 2 \mathrm{H}), 7.56-7.51(\mathrm{~m}, 3 \mathrm{H}), 7.50-7.45(\mathrm{~m}, 2 \mathrm{H}), 7.22(\mathrm{ddd}, J=8.0$, 6.4, $1.6 \mathrm{~Hz}, 1 \mathrm{H}), 6.13(\mathrm{~s}, 1 \mathrm{H}), 5.57(\mathrm{~s}, 1 \mathrm{H}), 3.97(\mathrm{~s}, 3 \mathrm{H}) ;{ }^{13} \mathrm{C}$ NMR (151 MHz, $\left.\mathbf{C D C l}_{3}\right) \delta 144.6,139.3,137.9,129.9\left(\mathrm{q},{ }^{2} J_{C-F}=32.4 \mathrm{~Hz}\right), 126.8,126.8$, 
$125.5\left(\mathrm{q},{ }^{3} J_{C-F}=3.9 \mathrm{~Hz}\right), 125.3,124.5\left(\mathrm{q},{ }^{1} J_{C-F}=271.8 \mathrm{~Hz}\right), 123.7\left(\mathrm{q},{ }^{2} J_{C-F}=35.3 \mathrm{~Hz}\right), 121.9\left(\mathrm{q},{ }^{1} J_{C-F}=\right.$ $270.2 \mathrm{~Hz}), 121.4,121.2,119.8,118.9\left(\mathrm{q},{ }^{3} J_{C-F}=3.0 \mathrm{~Hz}\right), 110.0,31.3\left(\mathrm{q},{ }^{4} J_{C-F}=2.6 \mathrm{~Hz}\right)$; IR (neat): $v_{\max }=$ 3060, 2929, 2852, 1469, 1323, 1252, 1159, 1106, 1075, 1064, 1015, $745 \mathrm{~cm}^{-1}$; HRMS m/z (EI) calc. for $\mathrm{C}_{19} \mathrm{H}_{13} \mathrm{~F}_{6} \mathrm{~N}\left[\mathrm{M}^{+}\right]$369.0952, found 369.0952; $\mathbf{R}_{f} 0.76$ (hexane/EtOAc, 4/1).

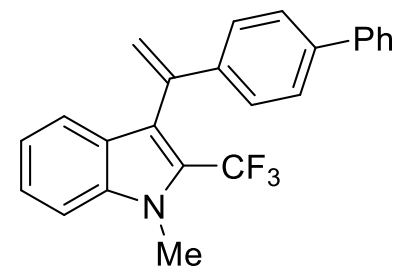

$3 a m$ (3-(1-([1,1'-biphenyl]-4-yl)vinyl)-1-methyl-2-(trifluoromethyl)-1Hindole (155 mg, 82\%): yellow liquid; ${ }^{1} \mathbf{H}$ NMR (600 MHz, $\left.\mathbf{C D C l}_{3}\right) \delta 7.68$ (dd, $J=8.1,1.3 \mathrm{~Hz}, 2 \mathrm{H}), 7.62-7.60(\mathrm{~m}, 3 \mathrm{H}), 7.54(\mathrm{~d}, J=8.5 \mathrm{~Hz}, 2 \mathrm{H}), 7.50(\mathrm{dd}, J$ $=8.1,7.6 \mathrm{~Hz}, 2 \mathrm{H}), 7.48-7.45(\mathrm{~m}, 2 \mathrm{H}), 7.41(\mathrm{td}, J=7.6,1.3 \mathrm{~Hz}, 1 \mathrm{H}), 7.23$ (ddd, $J=7.9,6.0,1.9 \mathrm{~Hz}, 1 \mathrm{H}), 6.15(\mathrm{~d}, J=1.2 \mathrm{~Hz}, 1 \mathrm{H}), 5.50(\mathrm{~d}, J=1.2 \mathrm{~Hz}$, 1H), 3.98 (s, 3H); ${ }^{13} \mathbf{C}$ NMR (151 MHz, $\left.\mathbf{C D C l}_{3}\right) \delta 140.9,140.6,139.9,139.8,137.8,129.0,127.5,127.2$, $127.0,126.9,125.1,123.6\left(\mathrm{q},{ }^{2} J_{C-F}=35.0 \mathrm{~Hz}\right), 122.1\left(\mathrm{q},{ }^{1} J_{C-F}=270.2 \mathrm{~Hz}\right), 121.7,121.0,119.7\left(\mathrm{q},{ }^{3} J_{C-F}=\right.$ $3.1 \mathrm{~Hz}), 117.6,109.9,31.3\left(\mathrm{q},{ }^{4} J_{C-F}=2.7 \mathrm{~Hz}\right.$ ); IR (neat): $v_{\max }=3056,3031,1251,1231,1171,1157$, 1101, 1074, 740, $696 \mathrm{~cm}^{-1}$; HRMS m/z (EI) calc. for $\mathrm{C}_{24} \mathrm{H}_{18} \mathrm{~F}_{3} \mathrm{~N}\left[\mathrm{M}^{+}\right]$377.1391, found 377.1389; $\mathbf{R}_{f} 0.70$ (hexane/EtOAc, 4/1).

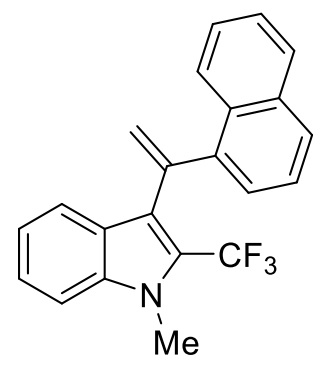

3an (1-methyl-3-(1-(naphthalen-1-yl)vinyl)-2-(trifluoromethyl)-1H-indole (93 mg, 53\%): yellow liquid; ${ }^{1} \mathbf{H}$ NMR $\left(\mathbf{6 0 0} \mathbf{~ M H z}, \mathbf{C D C l}_{3}\right) \delta 8.40(\mathrm{dd}, J=8.4,1.5 \mathrm{~Hz}, 1 \mathrm{H})$, $7.88(\mathrm{dd}, J=7.6,1.7 \mathrm{~Hz}, 1 \mathrm{H}), 7.79(\mathrm{dd}, J=7.9,1.2 \mathrm{~Hz}, 1 \mathrm{H}), 7.52(\mathrm{dd}, J=8.1,1.2$ $\mathrm{Hz}, 1 \mathrm{H}), 7.50-7.44(\mathrm{~m}, 2 \mathrm{H}), 7.41-7.35(\mathrm{~m}, 4 \mathrm{H}), 7.10(\mathrm{ddd}, J=8.0,6.4,1.5 \mathrm{~Hz}$, 1H), $5.89(\mathrm{~d}, J=1.8 \mathrm{~Hz}, 1 \mathrm{H}), 5.86(\mathrm{~d}, J=1.8 \mathrm{~Hz}, 1 \mathrm{H}), 3.89$ (s, 3H); ${ }^{13} \mathbf{C}$ NMR (151 MHz, $\left.\mathbf{C D C l}_{3}\right) \delta 140.36,138.85,137.79,134.27,131.37,128.63,128.04,126.81$, $126.77,126.15,125.96,125.74,125.26,125.05,123.00,122.96\left(\mathrm{q},{ }^{2} J_{C-F}=35.1 \mathrm{~Hz}\right), 122.10\left(\mathrm{q},{ }^{1} J_{C-F}=\right.$ $271.0 \mathrm{~Hz}), 121.82\left(\mathrm{q},{ }^{3} J_{C-F}=3.4 \mathrm{~Hz}\right), 121.72,121.06,109.86,31.38\left(\mathrm{q},{ }^{4} J_{C-F}=2.9 \mathrm{~Hz}\right) ; \mathbf{I R}($ neat $): v_{\max }=$ $3058,1469,1252,1233,1158,1105,1078,780,746 \mathrm{~cm}^{-1}$; HRMS m/z (EI) calc. for $\mathrm{C}_{22} \mathrm{H}_{16} \mathrm{~F}_{3} \mathrm{~N}\left[\mathrm{M}^{+}\right]$ 351.1235, found 351.1233; $\mathbf{R}_{f} 0.70$ (hexane/EtOAc, 4/1).

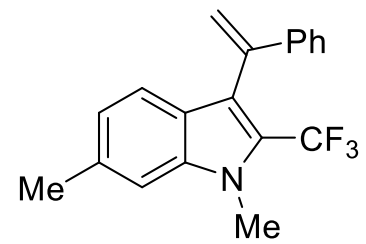

3 ba (1,6-dimethyl-3-(1-phenylvinyl)-2-(trifluoromethyl)-1H-indole (139 mg, 88\%): orange liquid; ${ }^{1} \mathbf{H}$ NMR $\left(\mathbf{6 0 0} \mathbf{M H z}, \mathbf{C D C l}_{3}\right) \delta 7.39(\mathrm{~d}, J=8.3 \mathrm{~Hz}, 2 \mathrm{H})$, $7.32-7.27(\mathrm{~m}, 5 \mathrm{H}), 7.23(\mathrm{~d}, J=8.5 \mathrm{~Hz}, 1 \mathrm{H}), 6.01(\mathrm{~s}, 1 \mathrm{H}), 5.38(\mathrm{~s}, 1 \mathrm{H}), 3.89(\mathrm{~s}$, 3H), 2.41 (s, 3H); ${ }^{13} \mathbf{C}$ NMR (151 MHz, $\left.\mathbf{C D C l}_{3}\right) \delta 141.0,140.3,136.3,130.3$, 128.4, 127.8, 127.2, 126.9, 126.5, $123.4\left(\mathrm{q},{ }^{2} J_{C-F}=35.2 \mathrm{~Hz}\right), 122.0\left(\mathrm{q},{ }^{1} J_{C-F}=270.0 \mathrm{~Hz}\right), 120.9,119.2(\mathrm{q}$, $\left.{ }^{3} J_{C-F}=3.2 \mathrm{~Hz}\right), 117.4\left(\mathrm{q},{ }^{4} J_{C-F}=1.6 \mathrm{~Hz}\right), 109.5,31.3\left(\mathrm{q},{ }^{4} J_{C-F}=2.6 \mathrm{~Hz}\right), 21.5 ;$ IR (neat): $v_{\max }=3031$, 
2923, 2855, 1255, 1228, 1171, 1152, 1102, 1075, 781, $702 \mathrm{~cm}^{-1} ; \mathbf{H R M S ~ m / z ~ ( E I ) ~ c a l c . ~ f o r ~} \mathrm{C}_{19} \mathrm{H}_{16} \mathrm{~F}_{3} \mathrm{~N}\left[\mathrm{M}^{+}\right]$ 315.1235, found 315.1237; $\mathbf{R}_{f} 0.77$ (hexane/EtOAc, 4/1).

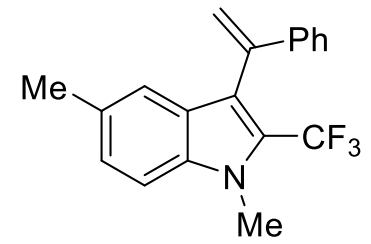

$3 \mathbf{c a}$ (1,5-dimethyl-3-(1-phenylvinyl)-2-(trifluoromethyl)-1H-indole (139 mg, 88\%): orange liquid; ${ }^{1} \mathbf{H}$ NMR (600 $\left.\mathbf{~ M H z , ~} \mathbf{C D C l}_{3}\right) \delta 7.49-7.45$ (m, 3H), $7.39-$ $7.34(\mathrm{~m}, 3 \mathrm{H}), 7.28(\mathrm{~s}, 1 \mathrm{H}), 7.06(\mathrm{~d}, J=8.2 \mathrm{~Hz}, 1 \mathrm{H}), 6.07(\mathrm{~d}, J=1.4 \mathrm{~Hz}, 1 \mathrm{H})$, $5.48(\mathrm{~d}, J=1.4 \mathrm{~Hz}, 1 \mathrm{H}), 3.94(\mathrm{~s}, 3 \mathrm{H}), 2.61(\mathrm{~s}, 3 \mathrm{H}) ;{ }^{13} \mathrm{C}$ NMR (151 MHz, $\left.\mathbf{C D C l}_{3}\right) \delta 141.1,140.4,138.2,135.3,128.4,127.8,126.5,125.0,122.9,122.9$ (q, $\left.J=35.4 \mathrm{~Hz}\right), 122.1$ (q, $J$ $=270.1 \mathrm{~Hz}), 121.3,119.8(\mathrm{q}, J=3.1 \mathrm{~Hz}), 117.4(\mathrm{q}, J=1.6 \mathrm{~Hz}), 109.6,31.1(\mathrm{q}, J=2.7 \mathrm{~Hz}), 22.2$; IR (neat): $v_{\max }=3031,2922,2855,1252,1229,1161,1100,1076,779,701 \mathrm{~cm}^{-1} ; \mathbf{H R M S ~ m} / \mathrm{z}$ (EI) calc. for $\mathrm{C}_{19} \mathrm{H}_{16} \mathrm{~F}_{3} \mathrm{~N}\left[\mathrm{M}^{+}\right]$315.1235, found 315.1237; $\mathbf{R}_{f} 0.73$ (hexane/EtOAc, 4/1).

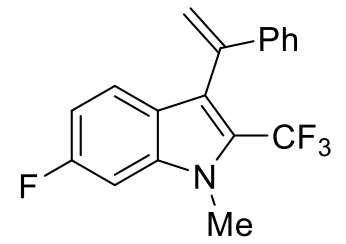

3da (6-fluoro-1-methyl-3-(1-phenylvinyl)-2-(trifluoromethyl)-1H-indole (136 mg, 85\%): yellow liquid; ${ }^{1} \mathbf{H}$ NMR $\left(600 \mathbf{~ M H z}, \mathbf{C D C l}_{3}\right) \delta 7.43\left(\mathrm{dd}, J=8.8 \mathrm{~Hz},{ }^{4} J_{H-F}=\right.$ $5.4 \mathrm{~Hz}, 1 \mathrm{H}), 7.41-7.38(\mathrm{~m}, 2 \mathrm{H}), 7.35-7.30(\mathrm{~m}, 3 \mathrm{H}), 7.10\left(\mathrm{dd}, J_{H-F}=9.7 \mathrm{~Hz}, J=\right.$ $2.2 \mathrm{~Hz}, 1 \mathrm{H}), 6.93\left(\mathrm{ddd}, J_{H-F}=9.1 \mathrm{~Hz}, J=2.2 \mathrm{~Hz}, 1 \mathrm{H}\right), 6.03(\mathrm{~d}, J=1.2 \mathrm{~Hz}, 1 \mathrm{H})$, $5.42(\mathrm{~d}, J=1.2 \mathrm{~Hz}, 1 \mathrm{H}), 3.87(\mathrm{~s}, 3 \mathrm{H}), ;{ }^{13} \mathbf{C}$ NMR (151 MHz, $\left.\mathbf{C D C l}_{3}\right) \delta 161.8\left(\mathrm{~d},{ }^{1} J_{C-F}=242.1 \mathrm{~Hz}\right), 140.8$ $\left(\mathrm{d},{ }^{4} J_{C-F}=1.3 \mathrm{~Hz}\right), 139.9,138.0\left(\mathrm{~d},{ }^{3} J_{C-F}=12.7 \mathrm{~Hz}\right), 128.5,128.0,126.5,123.9\left(\mathrm{qd},{ }^{2} J_{C-F}=35.5,{ }^{5} J_{C-F}=\right.$ $4.1 \mathrm{~Hz}), 123.5,123.0\left(\mathrm{~d},{ }^{3} J_{C-F}=10.4 \mathrm{~Hz}\right), 121.8\left(\mathrm{q},{ }^{1} J_{C-F}=270.2 \mathrm{~Hz}\right), 120.1\left(\mathrm{q},{ }^{3} J_{C-F}=3.3 \mathrm{~Hz}\right), 117.8(\mathrm{q}$, $\left.{ }^{4} J_{C-F}=1.6 \mathrm{~Hz}\right), 110.2\left(\mathrm{~d},{ }^{2} J_{C-F}=25.0 \mathrm{~Hz}\right), 96.1\left(\mathrm{~d},{ }^{2} J_{C-F}=26.7 \mathrm{~Hz}\right), 31.5\left(\mathrm{q},{ }^{4} J_{C-F}=2.7 \mathrm{~Hz}\right) ;$ IR (neat): $v_{\max }=3059,2954,2926,1472,1254,1229,1164,1106,827,702 \mathrm{~cm}^{-1} ; \mathbf{H R M S ~ m} / \mathrm{z}$ (EI) calc. for $\mathrm{C}_{18} \mathrm{H}_{13} \mathrm{~F}_{4} \mathrm{~N}\left[\mathrm{M}^{+}\right]$319.0984, found 319.0984; $\mathbf{R}_{f} 0.74$ (hexane/EtOAc, 4/1).

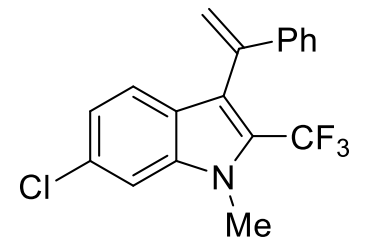

3ea (6-chloro-1-methyl-3-(1-phenylvinyl)-2-(trifluoromethyl)-1H-indole $\quad(146$ mg, 87\%): yellow liquid; ${ }^{1} \mathbf{H}$ NMR (600 $\left.\mathbf{~ M H z , ~} \mathbf{C D C l}_{3}\right) \delta 7.46(\mathrm{~d}, J=1.8 \mathrm{~Hz}$, $1 \mathrm{H}), 7.45(\mathrm{~d}, J=8.5 \mathrm{~Hz}, 1 \mathrm{H}), 7.43-7.41(\mathrm{~m}, 2 \mathrm{H}), 7.37-7.32(\mathrm{~m}, 3 \mathrm{H}), 7.16(\mathrm{dd}$, $J=8.5,1.8 \mathrm{~Hz}, 1 \mathrm{H}), 6.06(\mathrm{~d}, J=1.2 \mathrm{~Hz}, 1 \mathrm{H}), 5.44(\mathrm{~d}, J=1.2 \mathrm{~Hz}, 1 \mathrm{H}), 3.90(\mathrm{~s}$, 3H).; ${ }^{13} \mathbf{C}$ NMR (151 MHz, CDCl $) \delta 140.7,139.8,138.1,131.3,128.5,128.0,126.5,125.5,124.1$ (q, $\left.{ }^{2} J_{C-F}=35.4 \mathrm{~Hz}\right), 122.7,121.9,121.7\left(\mathrm{q},{ }^{1} J_{C-F}=270.5 \mathrm{~Hz}\right), 120.0\left(\mathrm{q},{ }^{3} J_{C-F}=3.3 \mathrm{~Hz}\right), 117.9\left(\mathrm{q},{ }^{4} J_{C-F}=1.6\right.$ $\mathrm{Hz}), 109.9,31.4\left(\mathrm{q},{ }^{4} J_{C-F}=2.6 \mathrm{~Hz}\right.$ ); IR (neat): $v_{\max }=3059,3029,2953,1469,1419,1251,1224,1173$, 1109, 1081, $700 \mathrm{~cm}^{-1}$; HRMS m/z (EI) calc. for $\mathrm{C}_{18} \mathrm{H}_{13} \mathrm{ClF}_{3} \mathrm{~N}\left[\mathrm{M}^{+}\right] 335.0689$, found 335.0691; $\mathbf{R}_{f} 0.74$ (hexane/EtOAc, 4/1). 


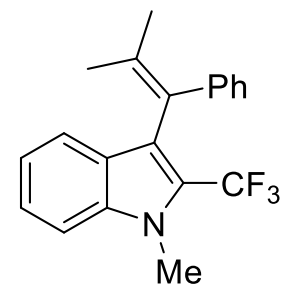

$3 f a$ (1-methyl-3-(2-methyl-1-phenylprop-1-en-1-yl)-2-(trifluoromethyl)- $1 H$-indole (148 mg, 90\%): white solid, m.p. 95-100 ${ }^{\circ} \mathrm{C} ;{ }^{1} \mathbf{H}$ NMR (600 MHz, $\left.\mathbf{C D C l}_{3}\right) \delta 7.59$ (d, $J=8.1 \mathrm{~Hz}, 1 \mathrm{H}), 7.38-7.37(\mathrm{~m}, 2 \mathrm{H}), 7.29-7.26(\mathrm{~m}, 4 \mathrm{H}), 7.20-7.16(\mathrm{~m}, 2 \mathrm{H}), 3.86$ (s, 3H), 1.99 (s, 3H), 1.71 (s, 3H); ${ }^{13} \mathbf{C}$ NMR (151 MHz, CDCl $) \delta 142.5,137.9$ (q, $\left.{ }^{4} J_{C-F}=1.3 \mathrm{~Hz}\right), 135.2\left(\mathrm{q},{ }^{4} J_{C-F}=1.6 \mathrm{~Hz}\right), 129.5,127.9,126.9,126.6,126.2,124.8$, $122.7\left(\mathrm{q},{ }^{2} J_{C-F}=34.5 \mathrm{~Hz}\right), 122.2\left(\mathrm{q},{ }^{1} J_{C-F}=270.0 \mathrm{~Hz}\right), 121.8,121.3\left(\mathrm{q},{ }^{3} J_{C-F}=3.6 \mathrm{~Hz}\right), 120.6,109.8,31.1$ $\left(\mathrm{q},{ }^{4} J_{C-F}=2.1 \mathrm{~Hz}\right), 23.0,22.0$; IR (neat): $v_{\max }=3056,2913,2855,1414,1252,1180,1156,1097,1073$, 742, $699 \mathrm{~cm}^{-1}$; HRMS m/z (EI) calc. for $\mathrm{C}_{20} \mathrm{H}_{18} \mathrm{~F}_{3} \mathrm{~N}\left[\mathrm{M}^{+}\right]$329.1391, found 329.1389; $\mathbf{R}_{f} 0.79$ (hexane/EtOAc, 4/1).

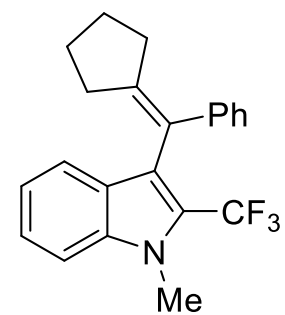

$3 g a$ (3-(cyclopentylidene(phenyl)methyl)-1-methyl-2-(trifluoromethyl)-1 $H$-indole (155 mg, 87\%): pale yellow liquid; ${ }^{1} \mathbf{H}$ NMR (600 $\left.\mathbf{M H z}, \mathbf{C D C l}_{3}\right) \delta 7.63$ (d, $J=7.8$ $\mathrm{Hz}, 1 \mathrm{H}), 7.44-7.42(\mathrm{~m}, 2 \mathrm{H}), 7.37(\mathrm{~d}, J=7.7 \mathrm{~Hz}, 2 \mathrm{H}), 7.31(\mathrm{dd}, J=7.6 \mathrm{~Hz}, 2 \mathrm{H})$, $7.25-7.19(\mathrm{~m}, 2 \mathrm{H}), 3.90(\mathrm{~s}, 3 \mathrm{H}), 2.72(\mathrm{t}, J=7.1 \mathrm{~Hz}, 2 \mathrm{H}), 2.28-2.12(\mathrm{~m}, 2 \mathrm{H}), 1.90-$ $1.75(\mathrm{~m}, 2 \mathrm{H}), 1.75-1.69(\mathrm{~m}, 2 \mathrm{H}) ;{ }^{13} \mathbf{C} \mathbf{N M R}\left(151 \mathbf{M H z}, \mathbf{C D C l}_{3}\right) \delta 147.5\left(\mathrm{q},{ }^{4} J_{C-F}=\right.$ $1.3 \mathrm{~Hz}), 142.6,138.1,128.4,127.9,126.1,126.1,124.8,123.3,122.5\left(\mathrm{q},{ }^{2} J_{C-F}=34.4 \mathrm{~Hz}\right), 122.2\left(\mathrm{q},{ }^{1} J_{C-F}\right.$ $=269.9 \mathrm{~Hz}), 121.6,121.4\left(\mathrm{q},{ }^{3} J_{C-F}=3.1 \mathrm{~Hz}\right), 120.7,109.8,33.3,33.2,31.1\left(\mathrm{q},{ }^{4} J_{C-F}=2.2 \mathrm{~Hz}\right), 27.6,26.2$; IR (neat): $v_{\max }=3055,2950,2666,2834,1415,1250,1155,1099,1082,742,697 \mathrm{~cm}^{-1} ;$ HRMS m/z (EI) calc. for $\mathrm{C}_{22} \mathrm{H}_{20} \mathrm{~F}_{3} \mathrm{~N}\left[\mathrm{M}^{+}\right]$355.1548, found 355.1550; $\mathbf{R}_{f} 0.76$ (hexane/EtOAc, 4/1).

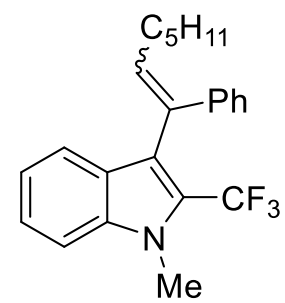

3 ha (1-methyl-3-(1-phenylhept-1-en-1-yl)-2-(trifluoromethyl)-1H-indole (163 mg, 88\%): pale yellow liquid; ${ }^{1} \mathbf{H}$ NMR (600 MHz, $\left.\mathbf{C D C l}_{3}\right) \delta 7.50(\mathrm{~d}, J=8.1 \mathrm{~Hz}, 2 \mathrm{H})$, $7.37-7.33(\mathrm{~m}, 2 \mathrm{H}), 7.31-7.26(\mathrm{~m}, 4 \mathrm{H}), 7.22(\mathrm{dd}, J=7.0 \mathrm{~Hz}, 1 \mathrm{H}), 7.12(\mathrm{ddd}, J=8.1$, 5.6, $2.3 \mathrm{~Hz}, 1 \mathrm{H}), 5.85(\mathrm{t}, J=7.5 \mathrm{~Hz}, 1 \mathrm{H}), 3.86(\mathrm{~s}, 3 \mathrm{H}), 2.39(\mathrm{dt}, J=7.5,7.4 \mathrm{~Hz}, 2 \mathrm{H})$, $1.51(\mathrm{tt}, J=7.47 .0, \mathrm{~Hz}, 2 \mathrm{H}), 1.37-1.31(\mathrm{~m}, 4 \mathrm{H}), 0.90(\mathrm{t}, J=7.1 \mathrm{~Hz}, 3 \mathrm{H}) ;{ }^{13} \mathbf{C} \mathbf{N M R}$ $\left(151 \mathrm{MHz}, \mathbf{C D C l}_{3}\right) \delta 140.6,137.7,135.4\left(\mathrm{q},{ }^{4} J_{C-F}=1.6 \mathrm{~Hz}\right), 131.6,129.3,128.0,127.2,126.8,124.8$, $123.0\left(\mathrm{q},{ }^{2} J_{C-F}=34.8,34.4 \mathrm{~Hz}\right), 122.8\left(\mathrm{q},{ }^{3} J_{C-F}=3.4 \mathrm{~Hz}\right), 122.2\left(\mathrm{~d},{ }^{4} J_{C-F}=270.1 \mathrm{~Hz}\right), 121.7,120.7,109.7$, 31.8, $31.3\left(\mathrm{q},{ }^{4} J_{C-F}=2.8 \mathrm{~Hz}\right.$ ), 29.9, 29.7, 22.7, 14.3; IR (neat): $v_{\max }=3057,2956,2926,2856,1468,1415$, 1261, 1171, 1158, 1105, 1085, $744 \mathrm{~cm}^{-1}$; HRMS m/z (EI) calc. for $\mathrm{C}_{23} \mathrm{H}_{24} \mathrm{~F}_{3} \mathrm{~N}\left[\mathrm{M}^{+}\right]$371.1861, found $371.1858 ; \mathbf{R}_{f} 0.76$ (hexane/EtOAc, 4/1). 


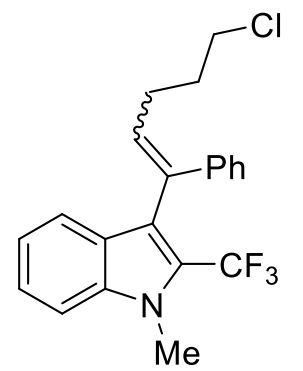

3ia (3-(5-chloro-1-phenylpent-1-en-1-yl)-1-methyl-2-(trifluoromethyl)-1 $H$-indole (166 mg, 88\%): pale yellow liquid; ${ }^{1} \mathbf{H}$ NMR (600 $\left.\mathbf{M H z}, \mathbf{C D C l}_{3}\right) \delta 7.49(\mathrm{~d}, J=8.1$ $\mathrm{Hz}, 1 \mathrm{H}), 7.39-7.36$ (m, 2H), 7.33 (dd, $J=8.3,6.6 \mathrm{~Hz}, 2 \mathrm{H}), 7.30$ (dd, $J=6.8,1.6 \mathrm{~Hz}$, 2H), 7.27 (dd, $J=6.8,1.9 \mathrm{~Hz}, 1 \mathrm{H}), 7.14$ (ddd, $J=7.9,5.1,2.7 \mathrm{~Hz}, 1 \mathrm{H}), 5.84(\mathrm{t}, J=$ $7.4 \mathrm{~Hz}, 1 \mathrm{H}), 3.87(\mathrm{~s}, 3 \mathrm{H}), 3.60(\mathrm{t}, J=6.7 \mathrm{~Hz}, 2 \mathrm{H}), 2.58(\mathrm{dt}, J=7.5 \mathrm{~Hz}, 2 \mathrm{H}), 2.00(\mathrm{tt}$, $J=6.8 \mathrm{~Hz}, 2 \mathrm{H}) ;{ }^{13} \mathbf{C} \mathbf{N M R}\left(\mathbf{1 5 1} \mathbf{M H z}, \mathbf{C D C l}_{3}\right) \delta 140.1,137.7,133.4,132.9\left(\mathrm{q},{ }^{4} J_{C-F}\right.$ $=1.6 \mathrm{~Hz}), 129.2,128.2,127.2,124.9,123.0\left(\mathrm{q},{ }^{2} J_{C-F}=34.5 \mathrm{~Hz}\right), 122.4\left(\mathrm{q},{ }^{3} J_{C-F}=2.9 \mathrm{~Hz}\right), 122.2\left(\mathrm{q},{ }^{1} J_{C-F}\right.$ $=270.3 \mathrm{~Hz}$ ), 121.5, 120.8, 109.8, 44.6, 33.1, 31.3 (q, ${ }^{4} J_{C-F}=2.8 \mathrm{~Hz}$ ), 27.1; IR (neat): $v_{\max }=3056,2955$, 1469, 1416, 1261, 1172, 1159, 1106, 745, $701 \mathrm{~cm}^{-1}$; HRMS m/z (EI) calc. for $\mathrm{C}_{21} \mathrm{H}_{19} \mathrm{ClF}_{3} \mathrm{~N}\left[\mathrm{M}^{+}\right]$ 377.1158, found 377.1160; $\mathbf{R}_{f} 0.67$ (hexane/EtOAc, 4/1).

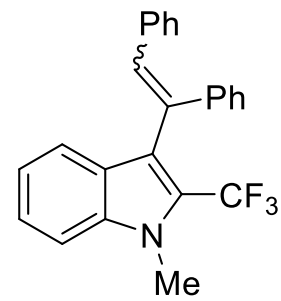

3ja (3-(1,2-diphenylvinyl)-1-methyl-2-(trifluoromethyl)-1H-indole (162 mg, 86\%): white solid, m.p. $120-125^{\circ} \mathrm{C} ;{ }^{1} \mathbf{H}$ NMR $\left(600 \mathbf{~ M H z} \mathbf{C D C l}_{3}\right) \delta 7.46(\mathrm{~d}, J=8.1 \mathrm{~Hz}, 1 \mathrm{H})$, $7.38(\mathrm{~d}, J=8.3 \mathrm{~Hz}, 1 \mathrm{H}), 7.35(\mathrm{ddd}, J=8.3,6.7,1.1 \mathrm{~Hz}, 1 \mathrm{H}), 7.24(\mathrm{dd}, \mathrm{J}=6.7,3.1 \mathrm{~Hz}$, 2H), $7.21-7.16(\mathrm{~m}, 8 \mathrm{H}), 7.09$ (ddd, $J=8.1,6.7,1.2 \mathrm{~Hz}, 1 \mathrm{H}), 6.77$ (s, 1H), 3.89 (s, $3 \mathrm{H}) ;{ }^{13} \mathbf{C}$ NMR (151 MHz, $\left.\mathbf{C D C l}_{3}\right) \delta 132.5\left(\mathrm{q},{ }^{4} J_{C-F}=2.3 \mathrm{~Hz}\right), 129.9,129.7,128.9$, $128.5,128.3,128.3,128.2,127.5,127.2,127.1,126.7,125.0,123.5\left(\mathrm{q},{ }^{2} J_{C-F}=34.4 \mathrm{~Hz}\right), 122.7\left(\mathrm{q},{ }^{3} J_{C-F}=\right.$ $3.0 \mathrm{~Hz}), 122.2\left(\mathrm{q},{ }^{1} J_{C-F}=270.3 \mathrm{~Hz}\right), 121.8,120.9,109.8,31.4$; IR (neat): $v_{\max }=3056,3024,2953,1468$, 1414, 1260, 1199, 1160, 1106, 1084, 745, $696 \mathrm{~cm}^{-1}$; HRMS m/z (EI) calc. for $\mathrm{C}_{24} \mathrm{H}_{18} \mathrm{~F}_{3} \mathrm{~N}\left[\mathrm{M}^{+}\right] 377.1391$, found 377.1394; $\mathbf{R}_{f} 0.63$ (hexane/EtOAc, 4/1).

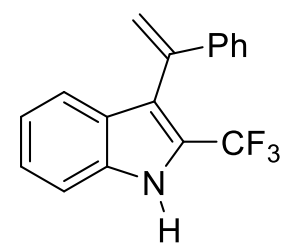

3 ka (3-(1-phenylvinyl)-2-(trifluoromethyl)- $1 H$-indole (122 mg, 85\%): yellow liquid; ${ }^{1}$ H NMR (600 MHz, CDCl $) \delta 8.43(\mathrm{~s}, 1 \mathrm{H}), 7.44(\mathrm{~d}, J=8.3 \mathrm{~Hz}, 1 \mathrm{H}), 7.39-7.35(\mathrm{~m}$, 2H), $7.34-7.27(\mathrm{~m}, 5 \mathrm{H}), 7.08(\mathrm{dd}, J=8.2,6.9 \mathrm{~Hz}, 1 \mathrm{H}), 5.93(\mathrm{~d}, J=1.2 \mathrm{~Hz}, 1 \mathrm{H})$, $5.46(\mathrm{~d}, J=1.2 \mathrm{~Hz}, 1 \mathrm{H}) ;{ }^{13} \mathbf{C}$ NMR $\left(\mathbf{1 5 1} \mathbf{~ M H z}, \mathbf{C D C l}_{3}\right) \delta 140.7,139.7,135.1,128.9$, $128.5,128.1,127.0,125.2,122.7\left(\mathrm{q},{ }^{2} J_{C-F}=36.6 \mathrm{~Hz}\right), 122.6,121.8,121.3,118.0\left(\mathrm{q},{ }^{4} J_{C-F}=1.6 \mathrm{~Hz}\right), 111.8$; IR (neat): $v_{\max }=3405,2960,2924,2853,1738,1455,1374,1326,1248,1168,1119,1045,732 \mathrm{~cm}^{-1}$; HRMS m/z (EI) calc. for $\mathrm{C}_{17} \mathrm{H}_{12} \mathrm{~F}_{3} \mathrm{~N}\left[\mathrm{M}^{+}\right]$287.0922, found 287.0924; $\mathbf{R}_{f}$ 0.51(hexane/EtOAc, 4/1).

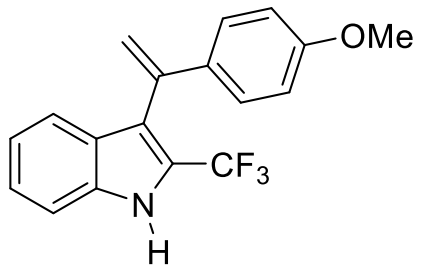

3kb 3-(1-(4-methoxyphenyl)vinyl)-2-(trifluoromethyl)-1H-indole (140 mg, 88\%): orange liquid; ${ }^{1} \mathbf{H}$ NMR (600 $\left.\mathbf{M H z}, \mathbf{C D C l}_{3}\right) \delta 8.53(\mathrm{~s}, 1 \mathrm{H}), 7.43(\mathrm{~d}, J$ $=8.4 \mathrm{~Hz}, 1 \mathrm{H}), 7.34-7.30(\mathrm{~m}, 4 \mathrm{H}), 7.10(\mathrm{ddd}, J=7.9,7.0,0.9 \mathrm{~Hz}, 1 \mathrm{H})$, $6.84(\mathrm{~d}, J=8.9 \mathrm{~Hz}, 2 \mathrm{H}), 5.85(\mathrm{~d}, J=1.2 \mathrm{~Hz}, 1 \mathrm{H}), 5.37(\mathrm{~d}, J=1.2 \mathrm{~Hz}, 1 \mathrm{H})$, $3.81(\mathrm{~s}, 3 \mathrm{H}) ;{ }^{13} \mathbf{C}$ NMR (151 MHz, $\left.\mathbf{C D C l}_{3}\right) \delta 159.4,138.8,134.9,133.1$, 
128.0, 127.5, 124.9, $122.4\left(\mathrm{q},{ }^{2} J_{C-F}=36.7 \mathrm{~Hz}\right), 121.7,121.6\left(\mathrm{q},{ }^{1} J_{C-F}=269.7 \mathrm{~Hz}\right), 121.0,119.6\left(\mathrm{q},{ }^{4} J_{C-F}=\right.$ $2.6 \mathrm{~Hz}), 115.9$, 113.6, 111.5, 55.2; IR (neat): $v_{\max }=3400,3062,2956,2838,1607,1510,1454,1326$, 1250, 1167, 1118, 1035, $748 \mathrm{~cm}^{-1}$; HRMS m/z (EI) calc. for $\mathrm{C}_{18} \mathrm{H}_{14} \mathrm{~F}_{3} \mathrm{NO}\left[\mathrm{M}^{+}\right]$317.1027, found 317.1027; $\mathbf{R}_{f} 0.47$ (hexane/EtOAc, 4/1).

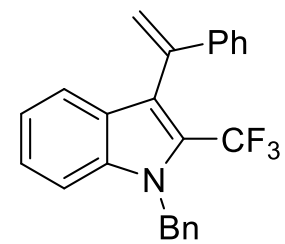

3la (1-benzyl-3-(1-phenylvinyl)-2-(trifluoromethyl)-1H-indole (166 mg, 88\%): pale yellow liquid; ${ }^{1} \mathbf{H}$ NMR (600 MHz, $\left.\mathbf{C D C l}_{3}\right) \delta 7.59(\mathrm{~d}, J=8.1 \mathrm{~Hz}, 1 \mathrm{H}), 7.48(\mathrm{dd}, J=$ $8.3,1.5 \mathrm{~Hz}, 2 \mathrm{H}), 7.39-7.32(\mathrm{~m}, 8 \mathrm{H}), 7.20(\mathrm{ddd}, J=8.1,6.5,1.5 \mathrm{~Hz}, 1 \mathrm{H}), 7.15(\mathrm{~d}, J$ $=7.4 \mathrm{~Hz}, 2 \mathrm{H}), 6.11(\mathrm{~d}, J=1.2 \mathrm{~Hz}, 1 \mathrm{H}), 5.60(\mathrm{~s}, 2 \mathrm{H}), 5.52(\mathrm{~d}, J=1.2 \mathrm{~Hz}, 1 \mathrm{H}) ;{ }^{13} \mathrm{C}$

NMR (151 MHz, $\left.\mathbf{C D C l}_{3}\right) \delta 140.9,140.2,137.7,137.2,129.0,128.5,127.9,127.7,127.3,126.5,126.1$, $125.4,123.6\left(\mathrm{q},{ }^{2} J_{C-F}=35.3 \mathrm{~Hz}\right), 122.0\left(\mathrm{q},{ }^{1} J_{C-F}=270.6 \mathrm{~Hz}\right), 121.7,121.3,120.4\left(\mathrm{q},{ }^{3} J_{C-F}=2.8 \mathrm{~Hz}\right)$, $117.8\left(\mathrm{q},{ }^{4} J_{C-F}=1.6 \mathrm{~Hz}\right), 110.8,48.6\left(\mathrm{q},{ }^{4} J_{C-F}=2.4 \mathrm{~Hz}\right) ; \mathbf{I R}\left(\right.$ neat) $: v_{\max }=3060,3033,1429,1249,1168$, 1102, 745, $695 \mathrm{~cm}^{-1}$; HRMS m/z (EI) calc. for $\mathrm{C}_{24} \mathrm{H}_{18} \mathrm{~F}_{3} \mathrm{~N}\left[\mathrm{M}^{+}\right]$377.1391, found 377.1393; $\mathbf{R}_{f} 0.69$ (hexane/EtOAc, 4/1).

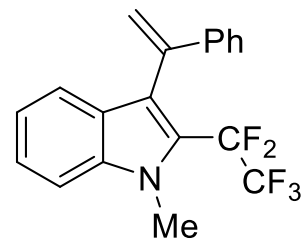

3 ma (1-methyl-2-(perfluoroethyl)-3-(1-phenylvinyl)-1H-indole (137 mg, 78\%): yellow solid, m.p. 80-85 ${ }^{\circ} \mathrm{C}$; ${ }^{1} \mathbf{H}$ NMR (600 MHz, $\left.\mathbf{C D C l}_{3}\right) \delta 7.53(\mathrm{~d}, J=8.0 \mathrm{~Hz}$, $1 \mathrm{H}), 7.47-7.40(\mathrm{~m}, 4 \mathrm{H}), 7.34-7.29(\mathrm{~m}, 3 \mathrm{H}), 7.20(\mathrm{ddd}, J=8.0,6.4,1.5 \mathrm{~Hz}, 1 \mathrm{H})$, $6.07(\mathrm{~d}, J=1.3 \mathrm{~Hz}, 1 \mathrm{H}), 5.40(\mathrm{~s}, 1 \mathrm{H}), 3.94(\mathrm{~s}, 3 \mathrm{H}),{ }^{13} \mathbf{C}$ NMR (151 MHz, CDCl 3$) \delta$ 141.1, 140.2, 138.5, 128.4, 127.7, 127.7, 126.5, 125.2, 122.6 (t, $J=3.4 \mathrm{~Hz}), 121.5,121.3$ (t, $J=27.3 \mathrm{~Hz}$ ), 121.1, 119.4 (qt, $J=286.7,39.9 \mathrm{~Hz}), 117.8,112.5$ (tq, $J=255.2,40.4 \mathrm{~Hz}), 110.0,31.9$ (tq, $J=5.5,2.6$ Hz); IR (neat): $v_{\max }=3058,2956,1467,1323,1198,1143,1117,1090,993,741 \mathrm{~cm}^{-1}$; HRMS m/z (EI) calc. for $\mathrm{C}_{19} \mathrm{H}_{14} \mathrm{~F}_{5} \mathrm{~N}\left[\mathrm{M}^{+}\right]$351.1046, found 351.1045; $\mathbf{R}_{f} 0.77$ (hexane/EtOAc, 4/1).

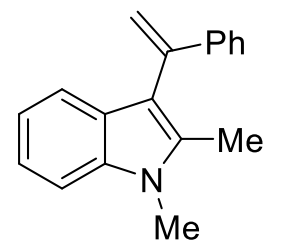

3na (1,2-dimethyl-3-(1-phenylvinyl)- $1 H$-indole ${ }^{\mathrm{S} 4}$ (106 mg, 86\%): orange liquid; ${ }^{1} \mathbf{H}$ NMR (600 MHz, $\left.\mathbf{C D C l}_{3}\right) \delta 7.41(\mathrm{dd}, J=6.7,1.4 \mathrm{~Hz}, 2 \mathrm{H}), 7.32-7.28(\mathrm{~m}, 4 \mathrm{H}), 7.25$ $(\mathrm{d}, J=8.5 \mathrm{~Hz}, 1 \mathrm{H}), 7.17(\mathrm{ddd}, J=8.2,6.8,1.0 \mathrm{~Hz}, 1 \mathrm{H}), 7.00(\mathrm{dd}, J=7.8,7.0 \mathrm{~Hz}, 1 \mathrm{H})$, $5.76(\mathrm{~d}, J=1.8 \mathrm{~Hz}, 1 \mathrm{H}), 5.32(\mathrm{~d}, J=1.8 \mathrm{~Hz}, 1 \mathrm{H}), 3.73(\mathrm{~s}, 3 \mathrm{H}), 2.29(\mathrm{~s}, 3 \mathrm{H}) ;{ }^{13} \mathbf{C} \mathbf{N M R}$ (151 MHz, $\left.\mathbf{C D C l}_{3}\right) \delta 143.0,142.3,136.8,135.1,128.4,127.6,127.5,121.0,120.6,119.8,119.5,119.4$, 115.1, 108.7, 29.9, 11.6; IR (neat): $v_{\max }=3052,2936,2912,1469,1445,1395,1333,1234,744 \mathrm{~cm}^{-1}$.

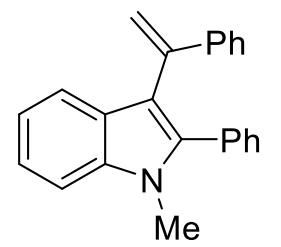

$30 a$ (1-methyl-2-phenyl-3-(1-phenylvinyl)-1H-indole (127 mg, 82\%): orange solid, m.p. 115-120 ${ }^{\circ} \mathrm{C} ;{ }^{1} \mathbf{H}$ NMR (600 $\left.\mathbf{~ M H z , ~} \mathbf{C D C l}_{3}\right) \delta 7.45$ - $7.41(\mathrm{~m}, 5 \mathrm{H}), 7.39-7.34(\mathrm{~m}$, 4H), 7.31 (ddd, $J=8.2,7.0,1.2 \mathrm{~Hz}, 1 \mathrm{H}), 7.26-7.23$ (m, 3H), 7.12 (ddd, $J=8.1,7.0$, 
$1.0 \mathrm{~Hz}, 1 \mathrm{H}), 5.64(\mathrm{~d}, J=1.7 \mathrm{~Hz}, 1 \mathrm{H}), 5.21(\mathrm{~d}, J=1.7 \mathrm{~Hz}, 1 \mathrm{H}), 3.73(\mathrm{~s}, 3 \mathrm{H}) ;{ }^{13} \mathbf{C} \mathbf{N M R}\left(\mathbf{1 5 1} \mathbf{M H z}, \mathbf{C D C l}_{3}\right)$ $\delta 142.5,142.0,139.4,137.4,132.1,130.8,128.3,128.1,128.0,127.8,127.4,127.4,122.1,120.6,120.0$, 116.5, 115.1, 109.6, 31.2; IR (neat): $v_{\max }=3054,3026,2925,1466,1443,1366,742,699 \mathrm{~cm}^{-1}$; HRMS $\mathrm{m} / \mathrm{z}$ (EI) calc. for $\mathrm{C}_{23} \mathrm{H}_{19} \mathrm{~N}\left[\mathrm{M}^{+}\right]$309.1517, found 309.1514; $\mathbf{R}_{f} 0.70$ (hexane/EtOAc, 4/1).

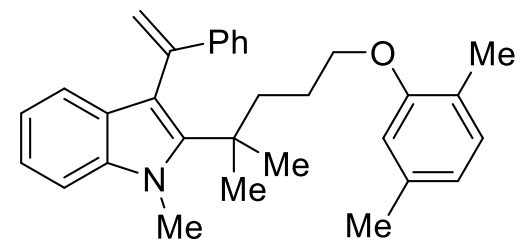

3pa (2-(5-(2,5-dimethylphenoxy)-2-methylpentan-2-yl)-1-methyl-3(1-phenylvinyl)-1H-indole (186 mg, 85\%): orange liquid; ${ }^{1} \mathbf{H}$ NMR $\left(600 \mathrm{MHz}, \mathbf{C D C l}_{3}\right) \delta{ }^{1} \mathbf{H}$ NMR (600 MHz, $\left.\mathbf{c d c l}_{3}\right) \delta 7.38-7.35(\mathrm{~m}$, $2 \mathrm{H}), 7.29(\mathrm{~d}, J=8.2 \mathrm{~Hz}, 1 \mathrm{H}), 7.25-7.19(\mathrm{~m}, 4 \mathrm{H}), 7.04$ (ddd, $J=7.8$, 6.9, $1.0 \mathrm{~Hz}, 1 \mathrm{H}), 6.99$ (d, $J=7.4 \mathrm{~Hz}, 1 \mathrm{H}), 6.64(\mathrm{~d}, J=7.8 \mathrm{~Hz}, 1 \mathrm{H}), 6.51(\mathrm{~s}, 1 \mathrm{H}), 6.03$ (d, $J=1.8 \mathrm{~Hz}, 1 \mathrm{H})$, $5.30(\mathrm{~d}, J=1.8 \mathrm{~Hz}, 1 \mathrm{H}), 3.95(\mathrm{~s}, 3 \mathrm{H}), 3.81-3.77(\mathrm{~m}, 2 \mathrm{H}), 2.26(\mathrm{~s}, 3 \mathrm{H}), 2.15(\mathrm{~s}, 3 \mathrm{H}), 2.00-1.95(\mathrm{~m}, 2 \mathrm{H})$, $1.44(\mathrm{~s}, 3 \mathrm{H}), 1.42$ (s, 3H), 1.27 - 1.25 (m, 2H); ${ }^{\mathbf{1 3}} \mathbf{C}$ NMR (151 MHz, CDCl $\left.\mathbf{~}_{3}\right) \delta$ 157.0, 142.2, 138.7, 138.6, 136.4, 131.1, 130.3, 129.4, 128.8, 128.2, 127.5, 127.4, 126.4, 124.0, 123.6, 121.8, 120.6, 116.3, 112.0, 107.8, 68.0, 47.9, 37.3, 25.8, 24.5, 24.2, 21.5, 21.5, 15.9; IR (neat): $v_{\max }=2924,2856,1509,1468$, 1264, 1157, 1130, 1041, $741 \mathrm{~cm}^{-1}$; HRMS m/z (EI) calc. for $\mathrm{C}_{31} \mathrm{H}_{35} \mathrm{NO}\left[\mathrm{M}^{+}\right]$437.2719, found 437.2718; $\mathbf{R}_{f} 0.71$ (hexane/EtOAc, 4/1).

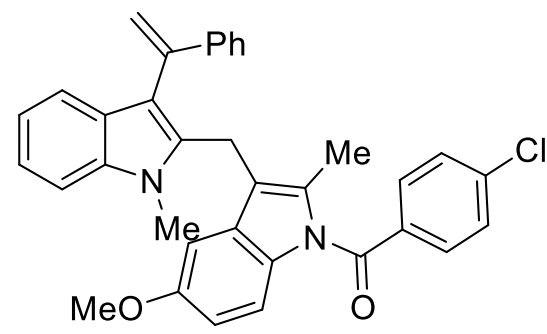

3qa ((4-chlorophenyl)(5-methoxy-2-methyl-3-((1-methyl-3-(1phenylvinyl)-1 $H$-indol-2-yl)methyl)-1 $H$-indol-1-yl)methanone $\quad(59$ mg, 22\%): pale orange liquid; ${ }^{1} \mathbf{H}$ NMR (600 $\left.\mathbf{M H z}, \mathbf{C D C l}_{3}\right) \delta 7.61$ $(\mathrm{d}, J=8.5 \mathrm{~Hz}, 2 \mathrm{H}), 7.47-7.43(\mathrm{~m}, 4 \mathrm{H}), 7.36(\mathrm{~d}, J=8.0 \mathrm{~Hz}, 1 \mathrm{H})$, $7.31-7.28(\mathrm{~m}, 4 \mathrm{H}), 7.21(\mathrm{ddd}, J=8.2,7.0,1.2 \mathrm{~Hz}, 1 \mathrm{H}), 7.05$ (ddd, $J=8.0,7.0,1.1 \mathrm{~Hz}, 1 \mathrm{H}), 6.83(\mathrm{dd}, J=9.0,1.7 \mathrm{~Hz}, 1 \mathrm{H}), 6.59(\mathrm{dd}, J$ = 9.0, $1.6 \mathrm{~Hz}, 1 \mathrm{H}), 6.40(\mathrm{~s}, 1 \mathrm{H}), 5.83(\mathrm{~d}, J=1.4 \mathrm{~Hz}, 1 \mathrm{H}), 5.43(\mathrm{~d}, J=1.4 \mathrm{~Hz}, 1 \mathrm{H}), 4.10(\mathrm{~s}, 2 \mathrm{H}), 3.60(\mathrm{~s}$, 3H), 3.45 (s, 3H), 2.26 (s, 3H); ${ }^{13} \mathbf{C}$ NMR (151 MHz, $\left.\mathbf{C D C l}_{3}\right) \delta 168.5,156.1,156.0,143.0,143.0,142.2$, 139.4, 137.1, 135.4, 134.4, 134.2, 131.3, 131.0, 130.7, 130.7, 129.3, 128.4, 127.8, 127.7, 127.5, 121.7, $120.1,119.8,116.0,115.8,115.3,114.9,112.1,109.0,101.2,55.5,30.3,22.1,13.4$; IR (neat): $v_{\max }=$ 3056, 2931, 2833, 1735, 1681, 1593, 1473, 1367, 1317, 1238, 1090, $744 \mathrm{~cm}^{-1}$; HRMS m/z (EI) calc. for $\mathrm{C}_{35} \mathrm{H}_{29} \mathrm{ClN}_{2} \mathrm{O}_{2}\left[\mathrm{M}^{+}\right]$544.1918, found 544.1914; $\mathbf{R}_{f} 0.29$ (hexane/EtOAc, 4/1).

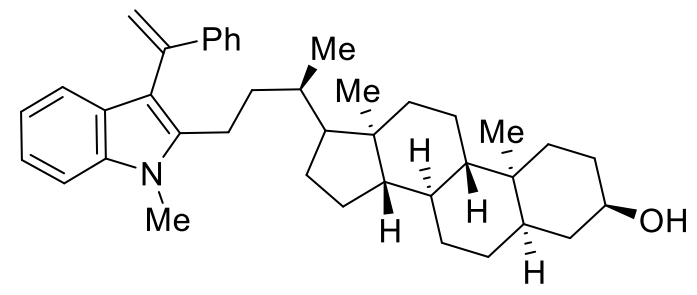

3ra $\quad((5 \mathrm{~S}, 8 \mathrm{~S}, 9 \mathrm{R}, 10 \mathrm{R}, 13 \mathrm{~S}, 14 \mathrm{~S}, 15 \mathrm{R})$-10,14-dimethyl-15((R)-4-(1-methyl-3-(1-phenylvinyl)-1H-indol-2-yl)butan-2yl)hexadecahydro-1H-cyclopenta[a]phenanthren-3-ol (228 mg, 81\%): orange solid, m.p. 135-140 ${ }^{\circ} \mathrm{C} ;{ }^{1} \mathbf{H}$ NMR (600 
MHz, $\left.\mathbf{C D C l}_{3}\right) \delta 7.67(\mathrm{~d}, J=8.0 \mathrm{~Hz}, 1 \mathrm{H}), 7.42(\mathrm{~d}, J=8.4 \mathrm{~Hz}, 1 \mathrm{H}), 7.40-7.37(\mathrm{~m}, 3 \mathrm{H}), 7.28(\mathrm{dd}, J=7.7$, $7.3 \mathrm{~Hz}, 2 \mathrm{H}), 7.23(\mathrm{t}, J=7.3 \mathrm{~Hz}, 1 \mathrm{H}), 7.15(\mathrm{ddd}, J=7.9,6.7,1.2 \mathrm{~Hz}, 1 \mathrm{H}), 6.02(\mathrm{~d}, J=1.3 \mathrm{~Hz}, 1 \mathrm{H}), 5.35(\mathrm{~d}$, $J=1.3 \mathrm{~Hz}, 1 \mathrm{H}), 4.70-4.65(\mathrm{~m}, 1 \mathrm{H}), 4.09(\mathrm{~s}, 3 \mathrm{H}), 1.83-1.73(\mathrm{~m}, 2 \mathrm{H}), 1.71-1.61(\mathrm{~m}, 2 \mathrm{H}), 2.05-2.01$ $(\mathrm{m}, 1 \mathrm{H}), 1.99-1.92(\mathrm{~m}, 1 \mathrm{H}), 1.83-1.73(\mathrm{~m}, 2 \mathrm{H}), 1.71-1.61(\mathrm{~m}, 2 \mathrm{H}), 1.45-1.07(\mathrm{~m}, 22 \mathrm{H}), 0.97(\mathrm{~d}, J=$ $6.5 \mathrm{~Hz}, 3 \mathrm{H}), 0.87$ (s, 3H), 0.66 (s, 3H); ${ }^{13} \mathbf{C}$ NMR (151 MHz, CDCl $) \delta 162.1,142.0,138.8,128.5,127.6$, 127.5, 126.4, 125.7, 125.4, 123.6, 121.8, 120.8, 115.9, 110.2, 75.3, 56.7, 56.7, 43.0, 42.2, 40.5, 40.3, 40.1, $36.1,35.7,35.2,34.9,32.2,31.7,30.2,29.9,28.6,27.3,26.5,26.1,24.5,23.5,21.0,18.8,12.3$; IR (neat): $v_{\max }=3525,3055,2932,2866,1703,1367,1245,1228,1132,1107,738 \mathrm{~cm}^{-1}$; HRMS m/z (EI) calc. for $\mathrm{C}_{40} \mathrm{H}_{53} \mathrm{NO}\left[\mathrm{M}^{+}\right]$563.4127, found 563.4124; $\mathbf{R}_{f} 0.77$ (hexane/EtOAc, 4/1).

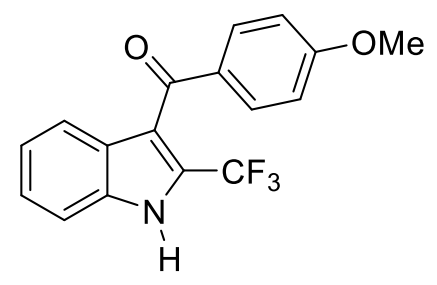

5 (4-methoxyphenyl)(2-(trifluoromethyl)-1H-indol-3-yl)methanone ${ }^{\mathrm{S5}} \quad(281$ mg, 88\%): pale yellow liquid; ${ }^{1} \mathbf{H}$ NMR (600 $\left.\mathbf{M H z}, \mathbf{C D C l}_{3}\right) \delta 8.91(\mathrm{~s}, 1 \mathrm{H})$, $7.88(\mathrm{~d}, J=8.9 \mathrm{~Hz}, 2 \mathrm{H}), 7.50(\mathrm{~d}, J=8.3 \mathrm{~Hz}, 1 \mathrm{H}), 7.43(\mathrm{~d}, J=8.1 \mathrm{~Hz}, 1 \mathrm{H})$, 7.37 (ddd, $J=8.3,7.0,1.1 \mathrm{~Hz}, 1 \mathrm{H}), 7.18$ (ddd, $J=8.1,7.0,1.0 \mathrm{~Hz}, 1 \mathrm{H})$, $6.95(\mathrm{~d}, J=8.9 \mathrm{~Hz}, 2 \mathrm{H}), 3.89$ (s, 3H); ${ }^{13} \mathbf{C}$ NMR (151 MHz, CDCl $)$ 190.2, 163.9, 134.6, 132.4, 131.6, 126.4, $126.3\left(\mathrm{q},{ }^{2} J_{C-F}=38.9 \mathrm{~Hz}\right), 125.3,122.3,121.9,120.8\left(\mathrm{q},{ }^{1} J_{C-F}=269.9\right.$ $\mathrm{Hz}), 117.5,113.8,112.2,55.5$; IR (neat): $v_{\max }=3313$, 2927, 2853, 2346, 1729, 1604, 1511, 1327, 1251, 1167, 1121, 1033, $748 \mathrm{~cm}^{-1}$; HRMS m/z (EI) calc. for $\mathrm{C}_{17} \mathrm{H}_{12} \mathrm{~F}_{3} \mathrm{NO}_{2}\left[\mathrm{M}^{+}\right]$319.0820, found 319.0816; $\mathbf{R}_{f}$ 0.57 (hexane/EtOAc, 4/1).

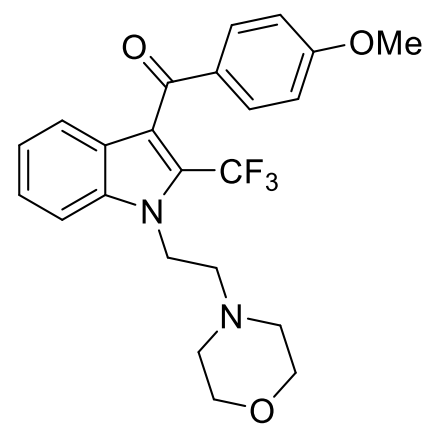

7 (4-methoxyphenyl)(1-(2-morpholinoethyl)-2-(trifluoromethyl)- $1 H$-indol3-yl)methanone (359 mg, 83\%): pale yellow liquid; ${ }^{1} \mathbf{H}$ NMR (600 MHz, $\left.\mathbf{C D C l}_{3}\right) \delta 7.84(\mathrm{~d}, J=8.9 \mathrm{~Hz}, 2 \mathrm{H}), 7.48(\mathrm{~d}, J=8.5 \mathrm{~Hz}, 1 \mathrm{H}), 7.43(\mathrm{~d}, J=8.2$ $\mathrm{Hz}, 1 \mathrm{H}), 7.38(\mathrm{dd}, J=8.5,6.9 \mathrm{~Hz}, 1 \mathrm{H}), 7.15(\mathrm{dd}, J=8.2,6.9 \mathrm{~Hz}, 1 \mathrm{H}), 6.91$ $(\mathrm{d}, J=8.9 \mathrm{~Hz}, 2 \mathrm{H}), 4.45(\mathrm{t}, J=7.7 \mathrm{~Hz}, 2 \mathrm{H}), 3.85(\mathrm{~s}, 3 \mathrm{H}), 3.72(\mathrm{t}, J=4.5 \mathrm{~Hz}$, 4H), 2.79 (t, $J=7.7 \mathrm{~Hz}, 2 \mathrm{H}), 2.57(\mathrm{t}, J=4.5 \mathrm{~Hz}, 4 \mathrm{H}) ;{ }^{13} \mathbf{C}$ NMR (151 MHz, $\left.\mathbf{C D C l}_{3}\right) \delta 190.5,164.1,136.9,132.3,131.6,125.9,125.6,124.6\left(\mathrm{q},{ }^{2} J_{C-F}=\right.$ $37.4 \mathrm{~Hz}), 122.1,122.0,121.3$ (q, $\left.{ }^{1} J_{C-F}=269.9 \mathrm{~Hz}\right), 118.7$ (q, $\left.{ }^{3} J_{C-F}=2.0 \mathrm{~Hz}\right), 113.9,110.6,67.0,57.7$, 55.7, 54.1, 43.0; IR (neat): $v_{\max }=2958,2853,1654,1598,1423,1253,1161,1114,1024,750 \mathrm{~cm}^{-1}$; HRMS m/z (EI) calc. for $\mathrm{C}_{23} \mathrm{H}_{23} \mathrm{~F}_{3} \mathrm{~N}_{2} \mathrm{O}_{3}\left[\mathrm{M}^{+}\right]$432.1661, found 432.1659; $\mathbf{R}_{f} 0.63$ (hexane/EtOAc, 1/4). 


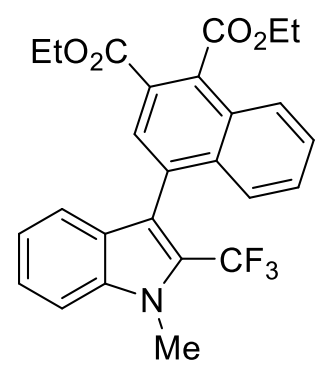

a (diethyl 4-(1-methyl-2-(trifluoromethyl)-1H-indol-3-yl)naphthalene-1,2dicarboxylate: white solid (82 mg, 87\%); ${ }^{1} \mathbf{H}$ NMR (600 $\left.\mathbf{M H z}, \mathbf{C D C l}_{3}\right) \delta 8.04$ (s, $1 \mathrm{H}), 7.97(\mathrm{ddd}, J=8.5,1.0 \mathrm{~Hz}, 1 \mathrm{H}), 7.64(\mathrm{~d}, J=8.6 \mathrm{~Hz}, 1 \mathrm{H}), 7.59(\mathrm{ddd}, J=8.3$, 6.8, $1.2 \mathrm{~Hz}, 1 \mathrm{H}), 7.51-7.46(\mathrm{~m}, 2 \mathrm{H}), 7.43$ (ddd, $J=8.4,6.7,1.4 \mathrm{~Hz}, 1 \mathrm{H}), 7.16-$ $7.10(\mathrm{~m}, 2 \mathrm{H}), 4.62(\mathrm{q}, J=7.2 \mathrm{~Hz}, 2 \mathrm{H}), 4.41(\mathrm{q}, J=7.1 \mathrm{~Hz}, 2 \mathrm{H}), 4.01(\mathrm{~s}, 3 \mathrm{H}), 1.50$ (t, $J=7.2 \mathrm{~Hz}, 3 \mathrm{H}), 1.38(\mathrm{t}, J=7.1 \mathrm{~Hz}, 3 \mathrm{H}) ;{ }^{13} \mathbf{C} \mathbf{N M R}\left(\mathbf{1 5 1} \mathbf{M H z}, \mathbf{C D C l}_{3}\right) \delta 169.2$, 165.7, 138.0, 135.5, 134.8, 132.9, 129.6, 128.4, 127.5 (d, $\left.{ }^{3} J_{C-F}=5.1 \mathrm{~Hz}\right), 126.4,126.3,125.2,124.4$, $123.6\left(\mathrm{q},{ }^{2} J_{C-F}=35.1 \mathrm{~Hz}\right), 123.1,121.7\left(\mathrm{q},{ }^{1} J_{C-F}=270.0 \mathrm{~Hz}\right), 120.9,116.4\left(\mathrm{q},{ }^{3} J_{C-F}=3.0 \mathrm{~Hz}\right), 109.6,62.0$, 61.7, $31.1\left(\mathrm{q},{ }^{4} J_{C-F}=2.2 \mathrm{~Hz}\right), 14.2$; IR (neat): $v_{\max }=2983,2926,2349,1726,1469,1258,1160,1113$, 1047, $748 \mathrm{~cm}^{-1}$; HRMS m/z (EI) calc. for $\mathrm{C}_{26} \mathrm{H}_{22} \mathrm{~F}_{3} \mathrm{NO}_{4}\left[\mathrm{M}^{+}\right]$469.1501, found 469.1502; $\mathbf{R}_{f} 0.47$ (hexane/EtOAc, 4/1).

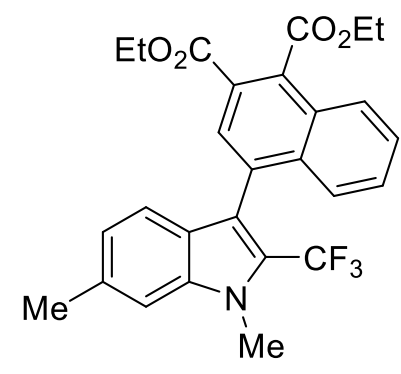

9b (diethyl 4-(1,6-dimethyl-2-(trifluoromethyl)-1H-indol-3-yl)naphthalene1,2-dicarboxylate: white solid (85 mg, 88\%); ${ }^{1} \mathbf{H}$ NMR (600 $\left.\mathbf{M H z}, \mathbf{C D C l}_{3}\right) \delta$ $8.03(\mathrm{~s}, 1 \mathrm{H}), 7.97(\mathrm{~d}, J=8.4 \mathrm{~Hz}, 1 \mathrm{H}), 7.65(\mathrm{~d}, J=8.4 \mathrm{~Hz}, 1 \mathrm{H}), 7.58(\mathrm{ddd}, J=$ 8.4, 6.8, $1.3 \mathrm{~Hz}, 1 \mathrm{H}), 7.47$ (ddd, $J=8.4,6.8,1.2 \mathrm{~Hz}, 1 \mathrm{H}), 7.28$ (s, 1H), 7.02 (d, $J=8.2 \mathrm{~Hz}, 1 \mathrm{H}), 6.95(\mathrm{dd}, J=8.2,1.4 \mathrm{~Hz}, 1 \mathrm{H}), 4.62(\mathrm{q}, J=7.2 \mathrm{~Hz}, 2 \mathrm{H}), 4.40$ (q, $J=7.1 \mathrm{~Hz}, 1 \mathrm{H}), 3.96(\mathrm{~s}, 3 \mathrm{H}), 2.53(\mathrm{~s}, 3 \mathrm{H}), 1.50(\mathrm{t}, J=7.2 \mathrm{~Hz}, 3 \mathrm{H}), 1.38(\mathrm{t}$, $J=7.1 \mathrm{~Hz}, 3 \mathrm{H}) ;{ }^{13} \mathbf{C}$ NMR (151 MHz, $\left.\mathbf{C D C l}_{3}\right) \delta 169.5,165.9,138.2,135.7,135.1,133.1,129.8,128.7$, $127.8,127.7,126.6,126.5,125.4,124.6,123.9\left(\mathrm{q},{ }^{2} J_{C-F}=35.5 \mathrm{~Hz}\right), 123.3,121.9\left(\mathrm{q},{ }^{1} J_{C-F}=270.0 \mathrm{~Hz}\right)$, 121.1, 109.8, 62.2, 61.9, 31.3, 22.3, 14.4; IR (neat): $v_{\max }=2982,2931,2349,1726,1420,1258,1169$, 1110, $767 \mathrm{~cm}^{-1}$; HRMS m/z (EI) calc. for $\mathrm{C}_{27} \mathrm{H}_{24} \mathrm{~F}_{3} \mathrm{NO}_{4}\left[\mathrm{M}^{+}\right]$483.1657, found 483.1660; $\mathbf{R}_{f} 0.53$ (hexane/EtOAc, 4/1).

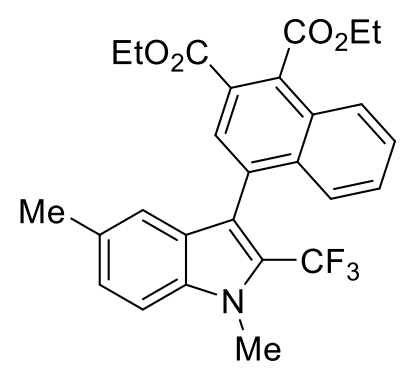

$9 c$ (diethyl 4-(1,5-dimethyl-2-(trifluoromethyl)-1H-indol-3-yl)naphthalene1,2-dicarboxylate: white solid (82 $\left.\mathrm{mg}, 85 \%) ;{ }^{1} \mathbf{H} \mathbf{~ N M R ~ ( 6 0 0 ~} \mathbf{M H z}, \mathbf{C D C l}_{3}\right) \delta$ $8.03(\mathrm{~s}, 1 \mathrm{H}), 7.98(\mathrm{~d}, J=8.5 \mathrm{~Hz}, 1 \mathrm{H}), 7.64(\mathrm{~d}, J=8.4 \mathrm{~Hz}, 1 \mathrm{H}), 7.59(\mathrm{ddd}, J=$ $8.5,6.8,1.3 \mathrm{~Hz}, 1 \mathrm{H}), 7.48(\mathrm{ddd}, J=8.4,6.8,1.2 \mathrm{~Hz}, 1 \mathrm{H}), 7.38(\mathrm{~d}, J=8.6 \mathrm{~Hz}$, 1H), $7.25(\mathrm{dd}, J=8.6,1.7 \mathrm{~Hz}, 1 \mathrm{H}), 6.90(\mathrm{~s}, 1 \mathrm{H}), 4.63(\mathrm{q}, J=7.2 \mathrm{~Hz}, 2 \mathrm{H})$, $4.41(\mathrm{q}, J=7.1 \mathrm{~Hz}, 2 \mathrm{H}), 3.97(\mathrm{~s}, 2 \mathrm{H}), 2.32(\mathrm{~s}, 2 \mathrm{H}), 1.50(\mathrm{t}, J=7.2 \mathrm{~Hz}, 3 \mathrm{H})$, $1.39(\mathrm{t}, J=7.1 \mathrm{~Hz}, 2 \mathrm{H}) ;{ }^{13} \mathbf{C}$ NMR (151 MHz, $\left.\mathbf{C D C l}_{3}\right) \delta 169.3,165.7,136.0,134.9,134.8,132.9,130.6$, 129.6, 128.5, $127.6\left(\mathrm{q},{ }^{3} J_{C-F}=2.6 \mathrm{~Hz}\right), 127.5,127.4,127.0,126.4,126.3,124.4,120.5,109.5,62.0,61.7$, 31.2, 21.2, 14.2, 14.2; IR (neat): $v_{\max }=2982,2925,2362,1726,1421,1260,1175,1111,1047,766 \mathrm{~cm}^{-1}$; HRMS m/z (EI) calc. for $\mathrm{C}_{27} \mathrm{H}_{24} \mathrm{~F}_{3} \mathrm{NO}_{4}\left[\mathrm{M}^{+}\right]$483.1657, found 483.1660; $\mathbf{R}_{f} 0.47$ (hexane/EtOAc, 4/1). 


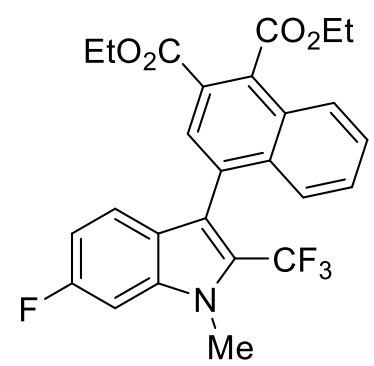

9d (diethyl 4-(6-fluoro-1-methyl-2-(trifluoromethyl)-1H-indol-3yl)naphthalene-1,2-dicarboxylate: white solid (83 mg, 85\%); ${ }^{1} \mathbf{H}$ NMR (300 MHz, CDCl 3$) \delta 8.02(\mathrm{~s}, 1 \mathrm{H}), 7.97(\mathrm{~d}, J=8.5 \mathrm{~Hz}, 1 \mathrm{H}), 7.62-7.58(\mathrm{~m}, 2 \mathrm{H})$, 7.49 (ddd, $J=8.2,6.8,1.2 \mathrm{~Hz}, 1 \mathrm{H}), 7.15$ (dd, $J=9.5,2.2 \mathrm{~Hz}, 1 \mathrm{H}), 7.07$ (dd, $J=$ 8.8, $5.3 \mathrm{~Hz}, 1 \mathrm{H}), 6.88(\mathrm{td}, J=9.0,2.2 \mathrm{~Hz}, 1 \mathrm{H}), 4.62(\mathrm{q}, J=7.2 \mathrm{~Hz}, 2 \mathrm{H}), 4.41(\mathrm{q}$, $J=7.1 \mathrm{~Hz}, 2 \mathrm{H}), 3.95$ (s, 3H); ${ }^{13} \mathbf{C}$ NMR (151 MHz, CDCl $) \delta 169.4,165.8$, $162.0\left(\mathrm{~d},{ }^{1} J_{C-F}=243.0 \mathrm{~Hz}\right), 135.3,135.0,132.4,129.9,128.8,127.9,127.8,126.5\left(\mathrm{~d},{ }^{2} J_{C-F}=35.2 \mathrm{~Hz}\right)$, $124.9\left(\mathrm{q},{ }^{2} J_{C-F}=36.0 \mathrm{~Hz}\right), 124.9\left(\mathrm{~d},{ }^{2} J_{C-F}=34.9 \mathrm{~Hz}\right), 124.6,124.0,122.9\left(\mathrm{~d},{ }^{4} J_{C-F}=10.3 \mathrm{~Hz}\right), 121.6(\mathrm{q}$, $\left.{ }^{1} J_{C-F}=270.2 \mathrm{~Hz}\right), 117.0\left(\mathrm{q},{ }^{4} J_{C-F}=3.2 \mathrm{~Hz}\right), 110.8\left(\mathrm{~d},{ }^{3} J_{C-F}=25.2 \mathrm{~Hz}\right), 96.4\left(\mathrm{~d},{ }^{3} J_{C-F}=26.8 \mathrm{~Hz}\right), 62.3,62.0$, 31.7 (q, $\left.{ }^{4} J_{C-F}=2.5 \mathrm{~Hz}\right), 14.4$; IR (neat): $v_{\max }=2986,2349,1725,1420,1258,1168,1109,1083,768 \mathrm{~cm}^{-1}$; HRMS m/z (EI) calc. for $\mathrm{C}_{26} \mathrm{H}_{21} \mathrm{~F}_{4} \mathrm{NO}_{4}\left[\mathrm{M}^{+}\right]$487.1407, found 487.1405; $\mathbf{R}_{f} 0.50$ (hexane/EtOAc, 4/1).

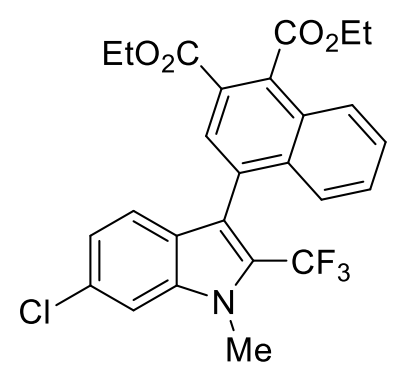

9e (diethyl 4-(6-chloro-1-methyl-2-(trifluoromethyl)-1H-indol-3yl)naphthalene-1,2-dicarboxylate: white solid (81 mg, 80\%); ${ }^{1} \mathbf{H}$ NMR (600 MHz, $\left.\mathbf{C D C l}_{3}\right) \delta 8.02(\mathrm{~s}, 1 \mathrm{H}), 7.97(\mathrm{~d}, J=8.5 \mathrm{~Hz}, 1 \mathrm{H}), 7.61-7.58(\mathrm{~m}, 2 \mathrm{H})$, $7.50-7.47(\mathrm{~m}, 2 \mathrm{H}), 7.08(\mathrm{dd}, J=8.5,1.7 \mathrm{~Hz}, 1 \mathrm{H}), 7.05(\mathrm{~d}, J=8.5 \mathrm{~Hz}, 1 \mathrm{H})$, $4.62(\mathrm{q}, J=7.2 \mathrm{~Hz}, 2 \mathrm{H}), 4.41(\mathrm{q}, J=7.1 \mathrm{~Hz}, 2 \mathrm{H}), 3.97$ (s, 3H), 1.50 (t, $J=7.2$ $\mathrm{Hz}, 3 \mathrm{H}), 1.39$ (t, $J=7.1 \mathrm{~Hz}, 3 \mathrm{H}) ;{ }^{13} \mathbf{C} \mathbf{N M R}\left(\mathbf{1 5 1} \mathbf{M H z}, \mathbf{C D C l}_{\mathbf{3}}\right) \delta 169.3,165.8$, $138.1,135.4,134.9,132.2,131.7,129.9,128.9,127.9,126.7,126.3,126.0,125.1$ (q, $\left.{ }^{2} J_{C-F}=35.5 \mathrm{~Hz}\right)$, 124.6, 122.6, $122.4,121.6\left(\mathrm{q},{ }^{1} J_{C-F}=270.4 \mathrm{~Hz}\right), 116.9\left(\mathrm{q},{ }^{3} J_{C-F}=2.9 \mathrm{~Hz}\right), 110.1,62.3,62.0,31.7\left(\mathrm{q},{ }^{4} J_{C-F}\right.$ $=2.6 \mathrm{~Hz}), 14.4$; IR (neat): $v_{\max }=2983,2939,2359,1737,1373,1235,1044,786,608 \mathrm{~cm}^{-1} ; \mathbf{H R M S ~ m} / \mathrm{z}$ (EI) calc. for $\mathrm{C}_{26} \mathrm{H}_{21} \mathrm{ClF}_{3} \mathrm{NO}_{4}\left[\mathrm{M}^{+}\right]$503.1111, found 503.1111; $\mathbf{R}_{f} 0.46$ (hexane/EtOAc, 4/1).

\section{References}

(S1) T. Shimada, I. Nakamura, Y. Yamamoto, J. Am. Chem. Soc. 2004, 126, 10546-10547

(S2) L. Pan, J. Elmasry, T. Osccorima, M. V. Cooke, S. Laulhé, Org. Lett. 2021, 23, 3389-3393

(S3) A. Arcadi, S. Cacchi, V. Carnicelli, F. Marinelli, Tetrahedron, 1994, 50, 437-452

(S4) T. Tsuchimoto, M. Kanbara, Org. Lett. 2011, 13, 912-915

(S5) Z. Chen, J. Zhu, H. Xie, S. Li, Y. Wu, Y. Gong, Synlett, 2010, 9, 1418 

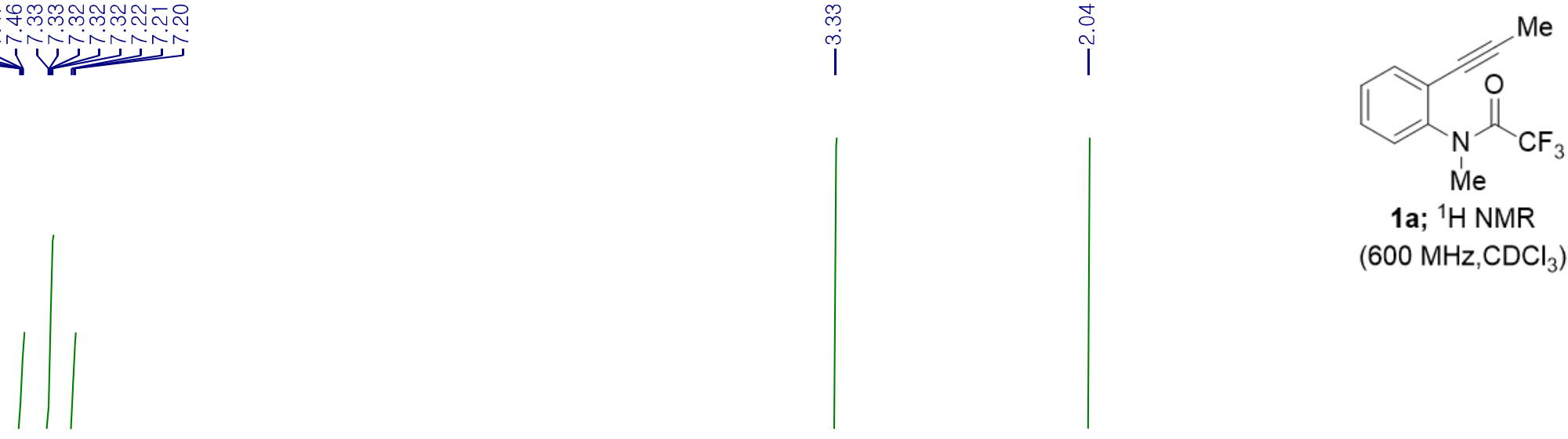

1a; ${ }^{1} \mathrm{H}$ NMR

$\left(600 \mathrm{MHz}, \mathrm{CDCl}_{3}\right)$

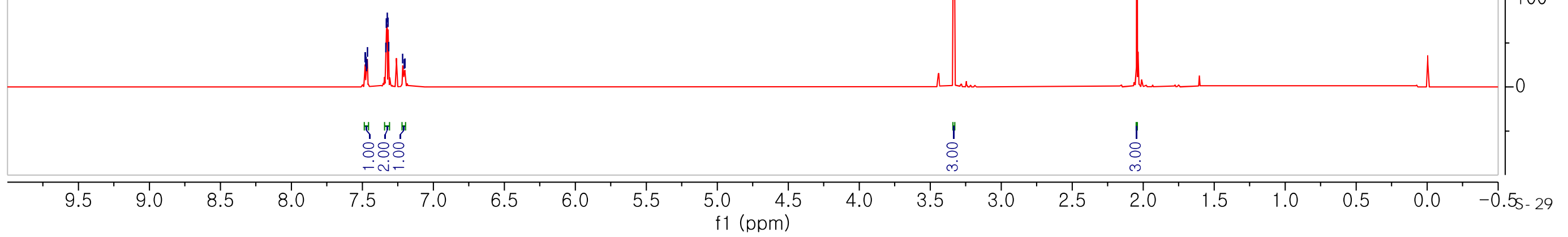




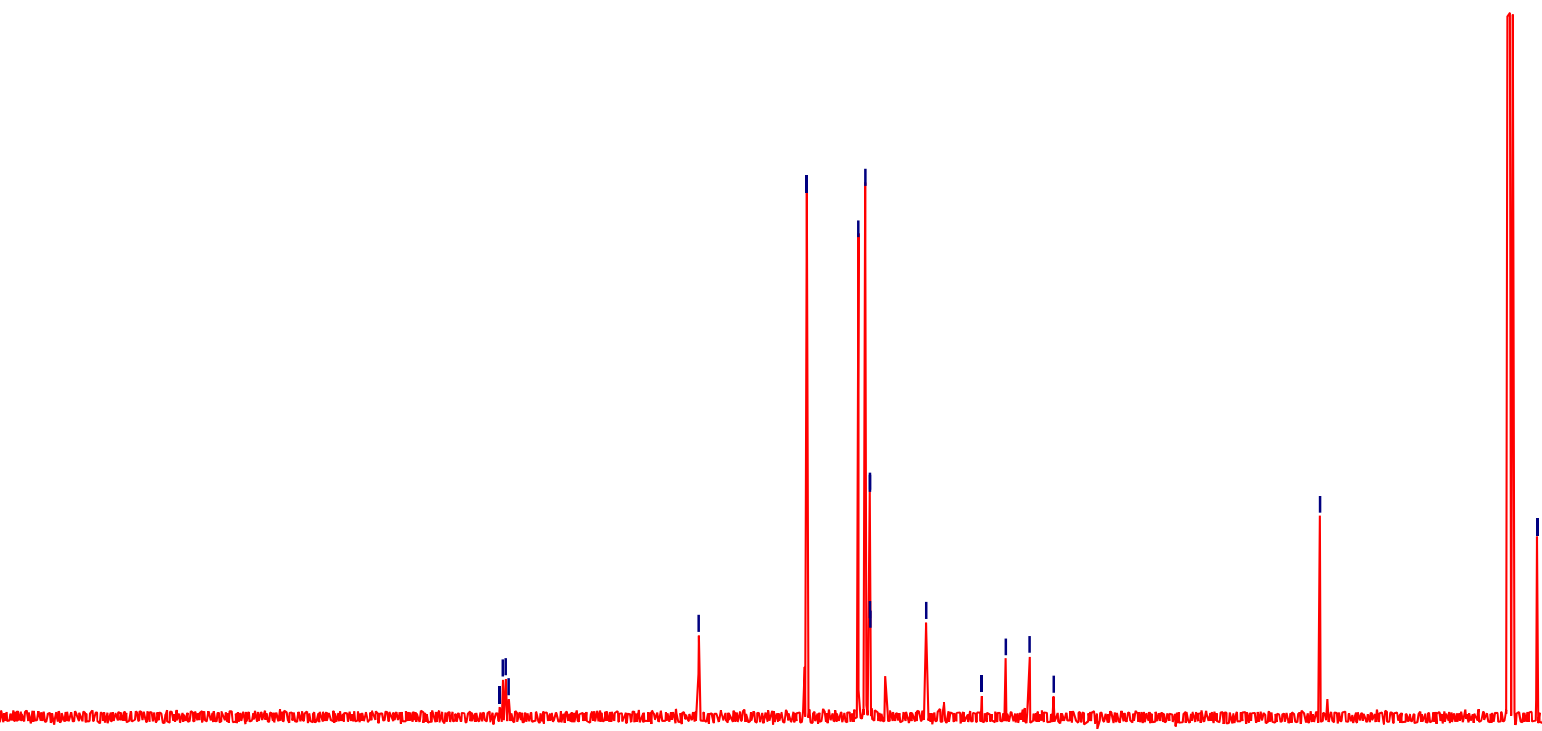




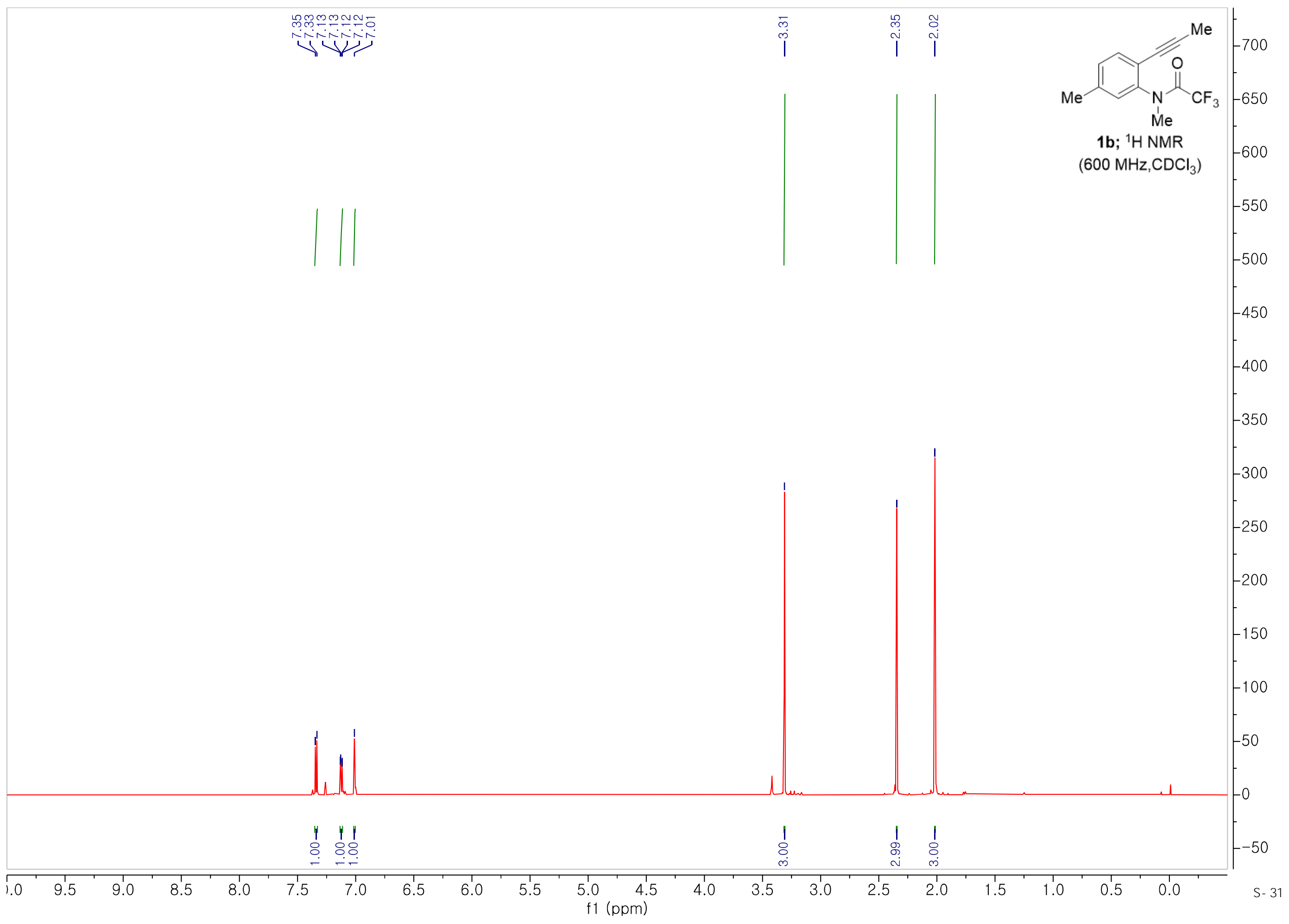




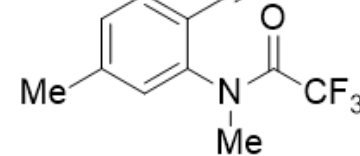

1b; ${ }^{13} \mathrm{C}$ NMR

$\left(151 \mathrm{MHz}, \mathrm{CDCl}_{3}\right.$ )
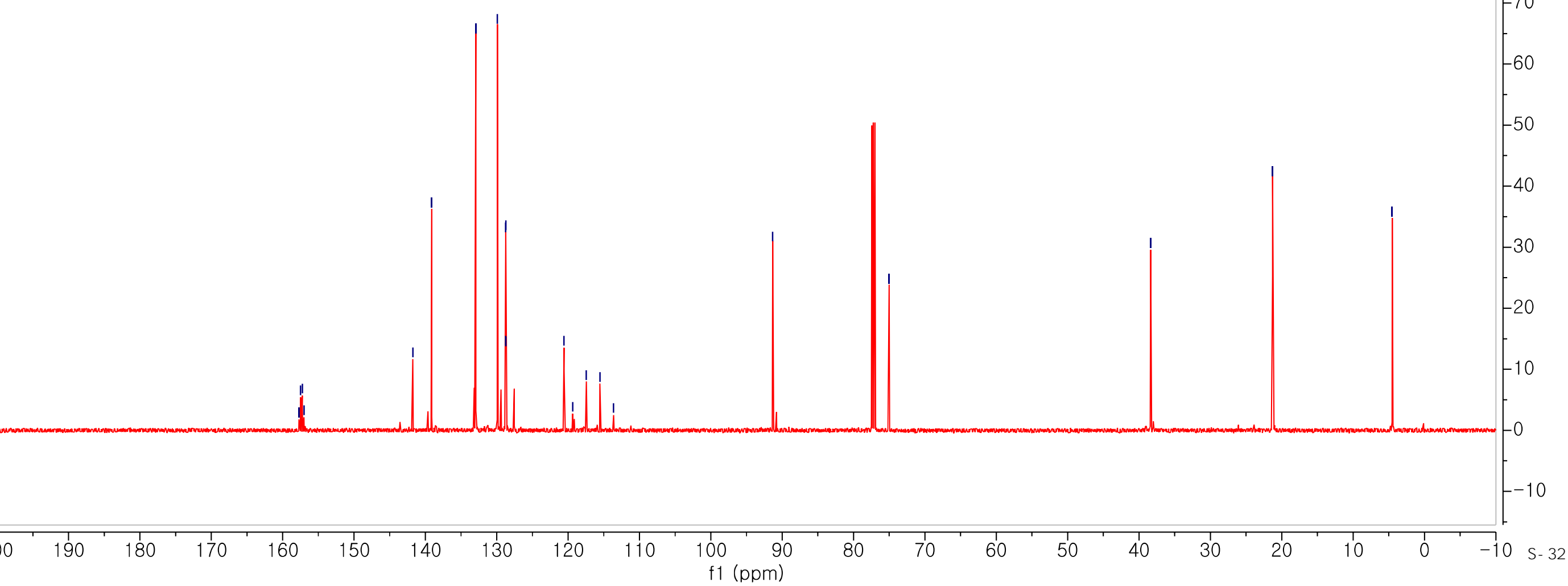
1c; ${ }^{1} \mathrm{H}$ NMR

$\left(600 \mathrm{MHz}, \mathrm{CDCl}_{3}\right.$ )

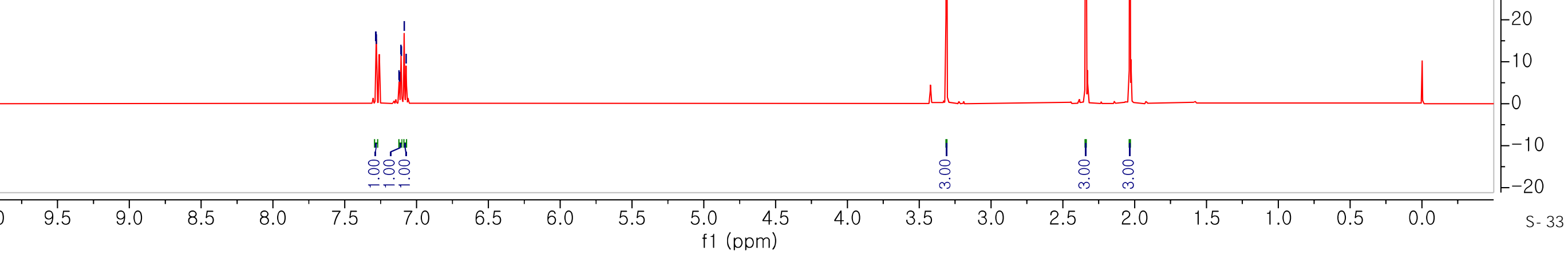




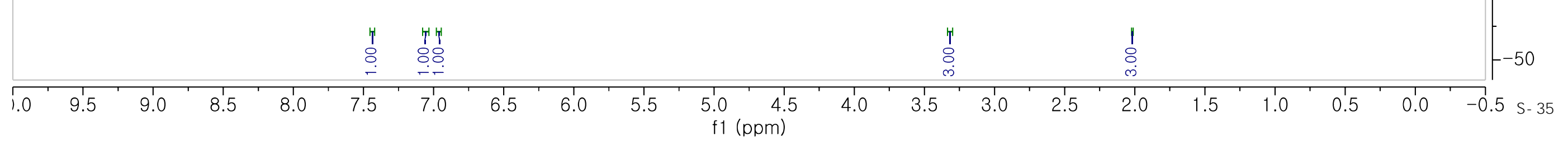



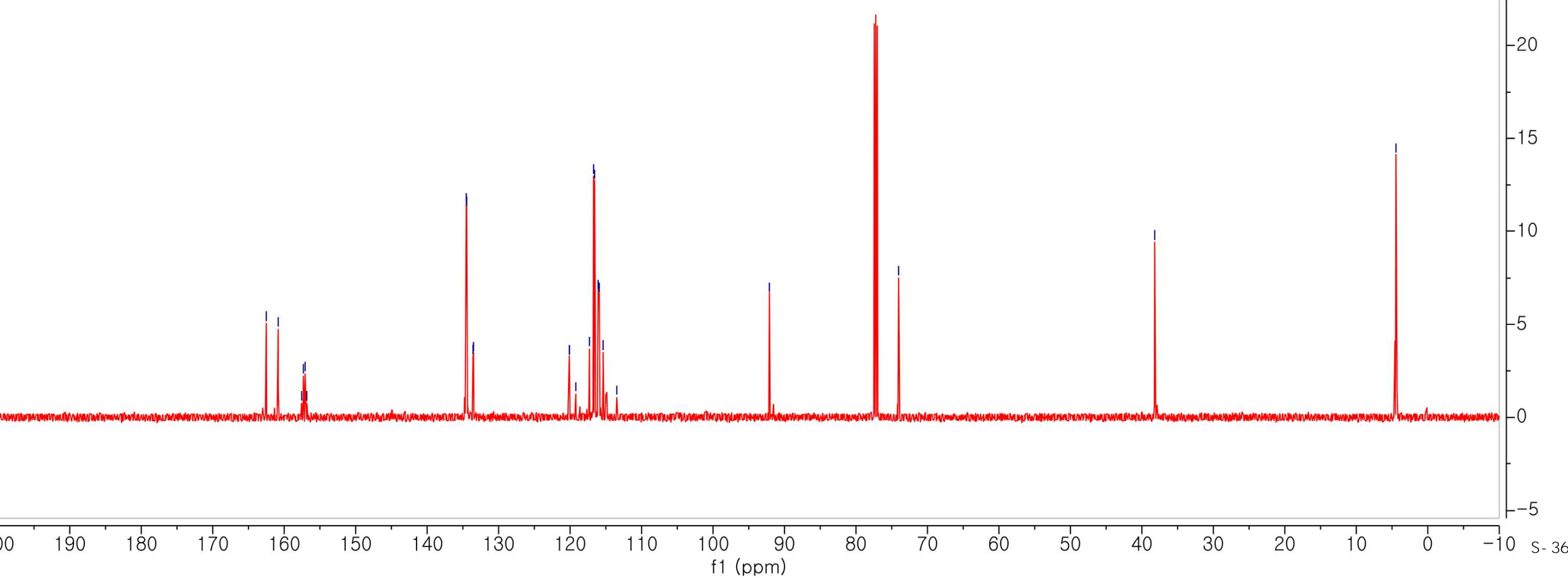


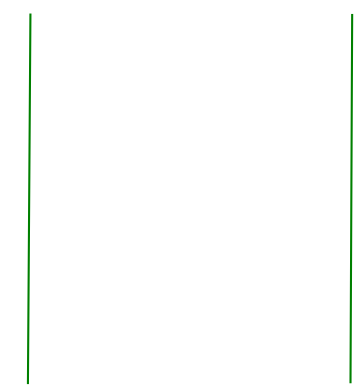

$$
\mid
$$

$\overbrace{\mathrm{Me}}^{\mathrm{Me}}$

1e; ${ }^{1} \mathrm{H}$ NMR (600 $\mathrm{MHz}, \mathrm{CDCl}_{3}$ ) 

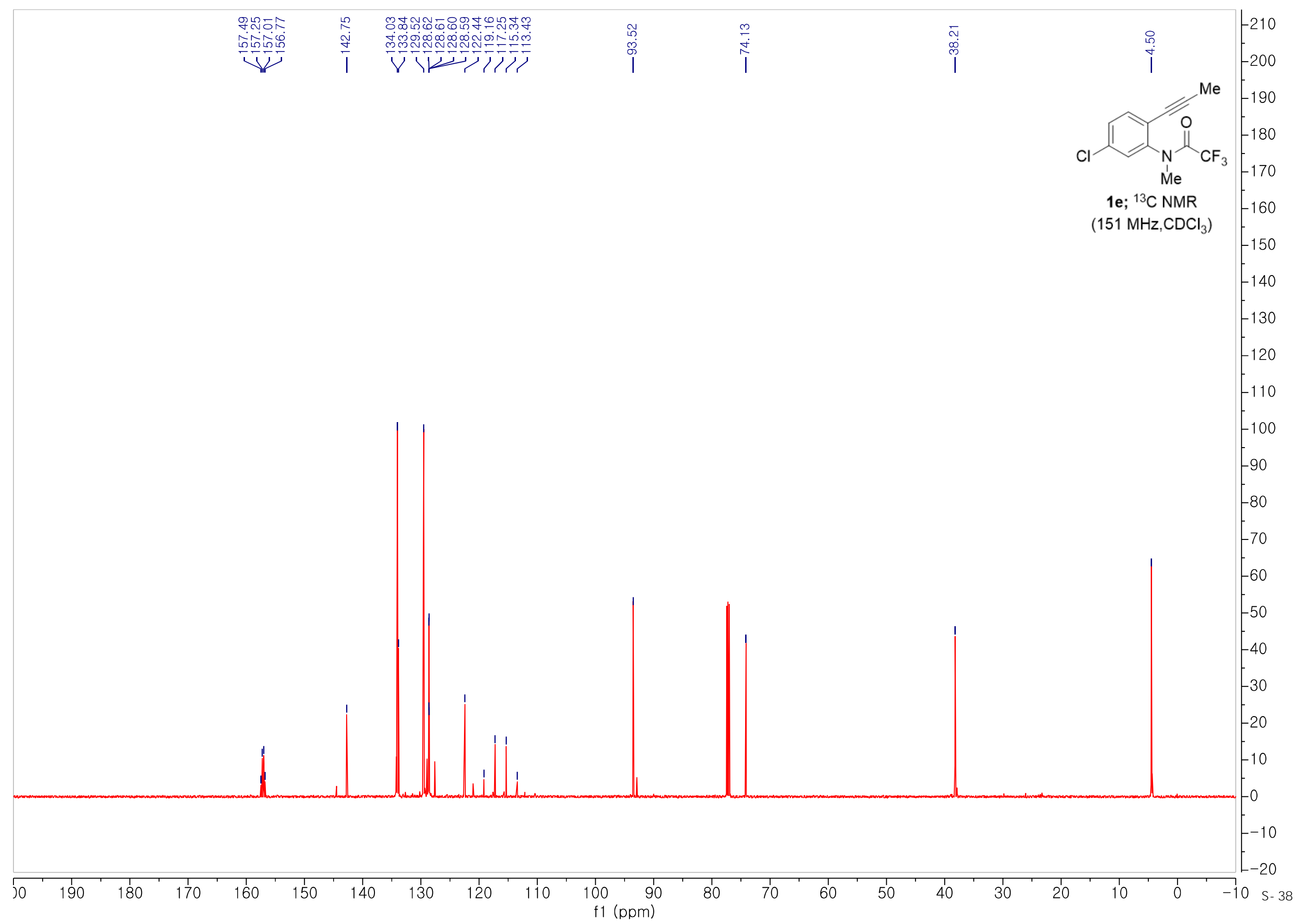


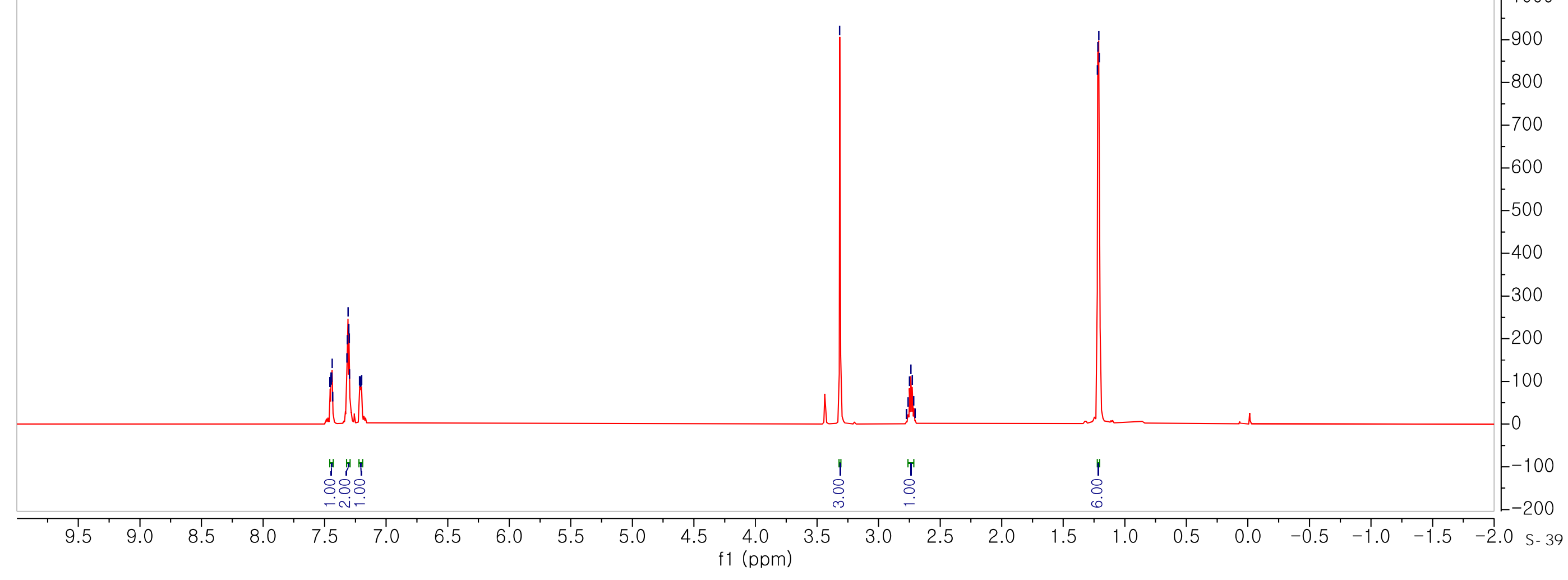



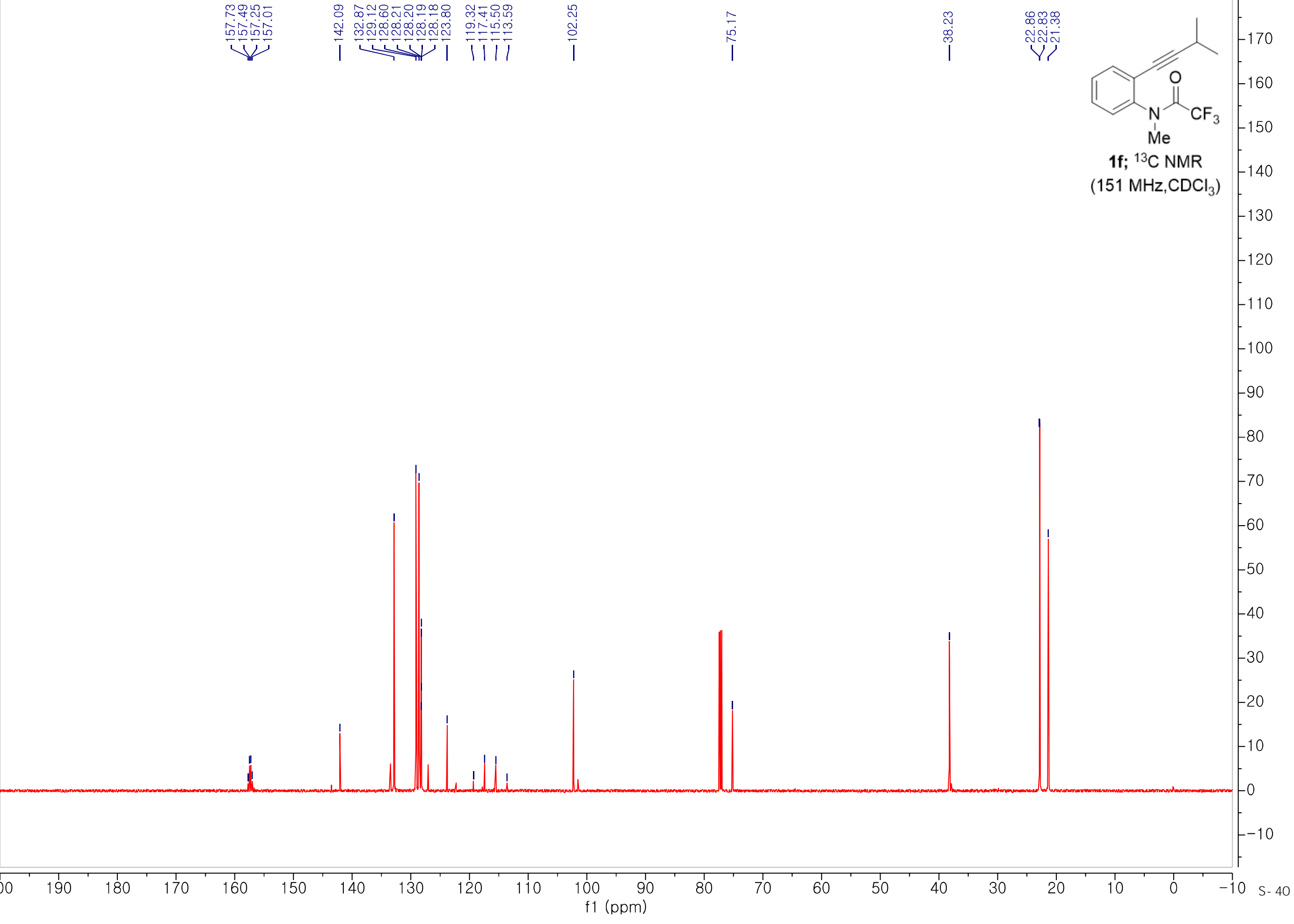

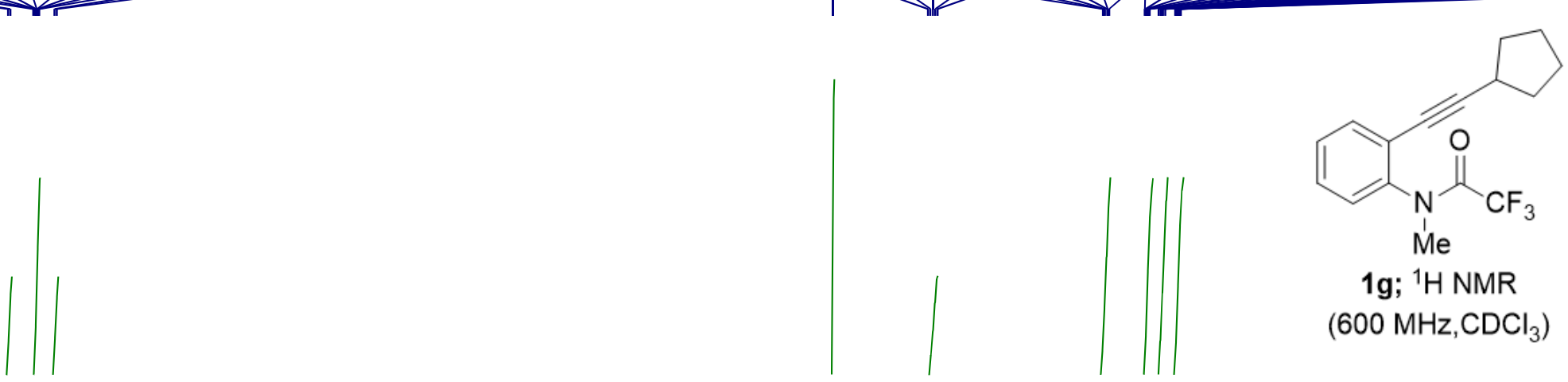

1g; ${ }^{1} \mathrm{H}$ NMR $\left(600 \mathrm{MHz}, \mathrm{CDCl}_{3}\right)$

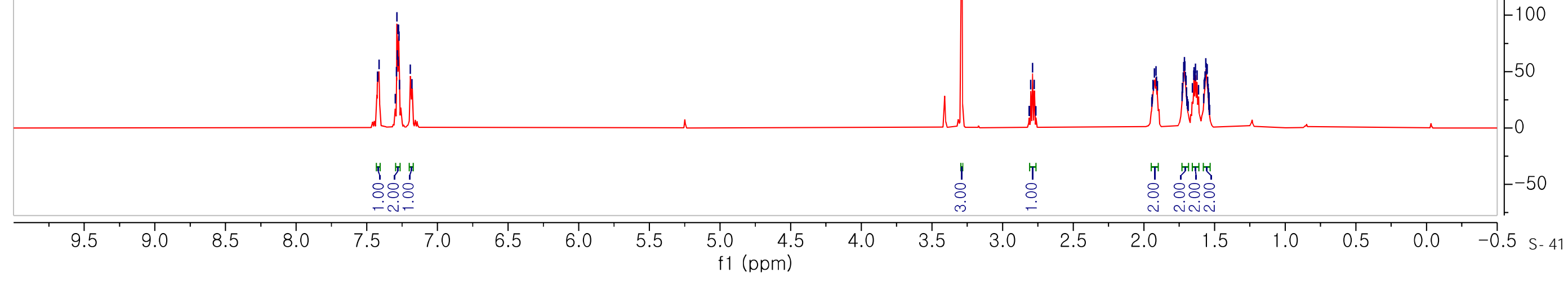




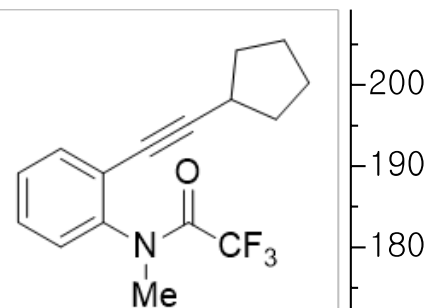
$\mathrm{Me}$

1g; ${ }^{13} \mathrm{C}$ NMR

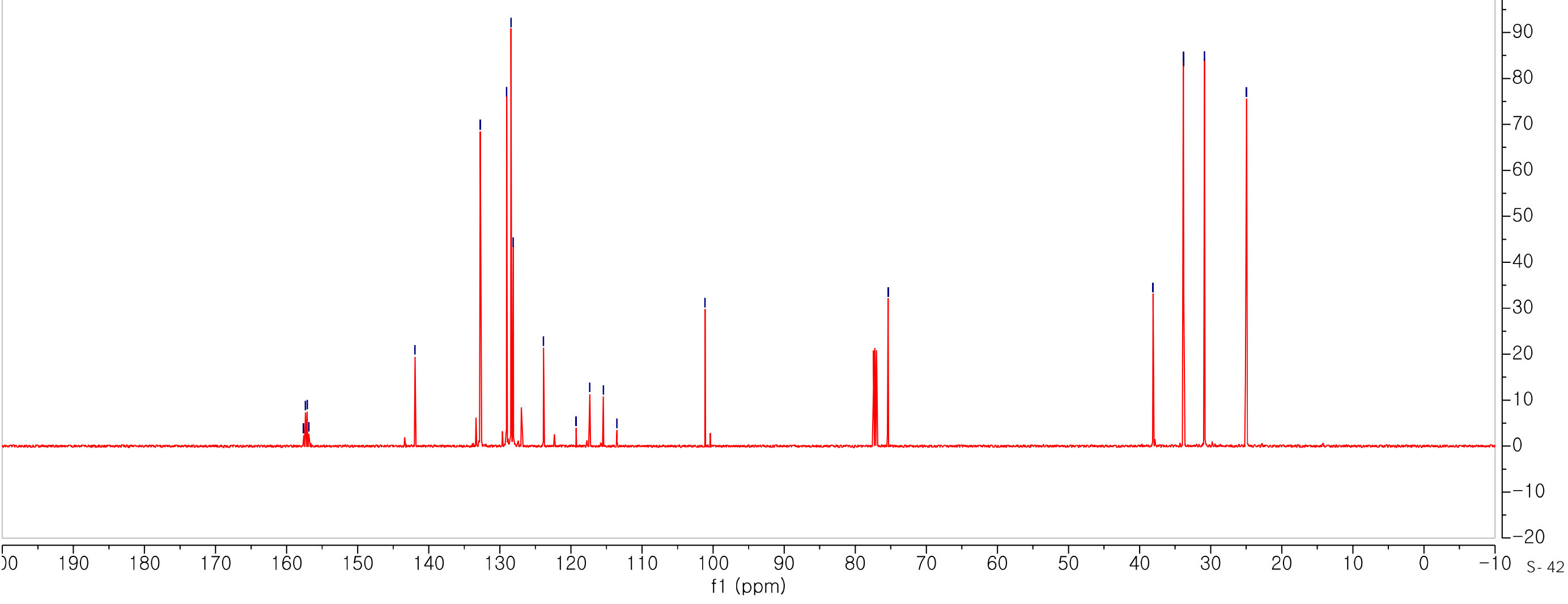



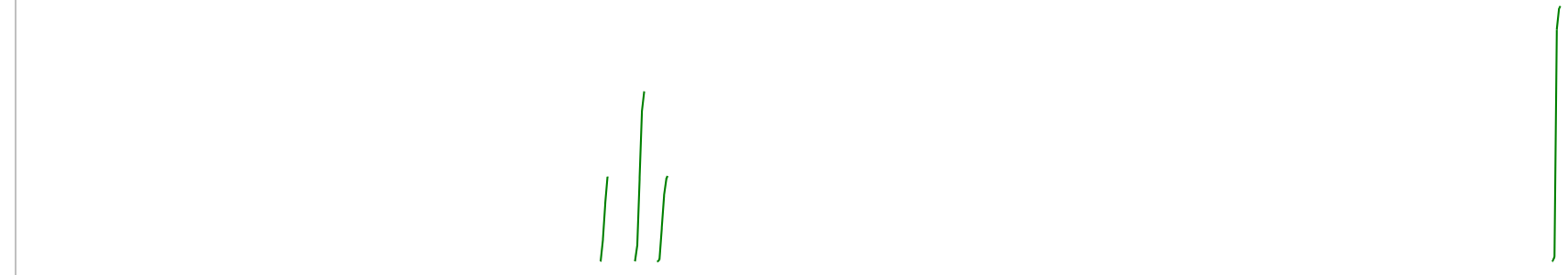

$$
\mid
$$
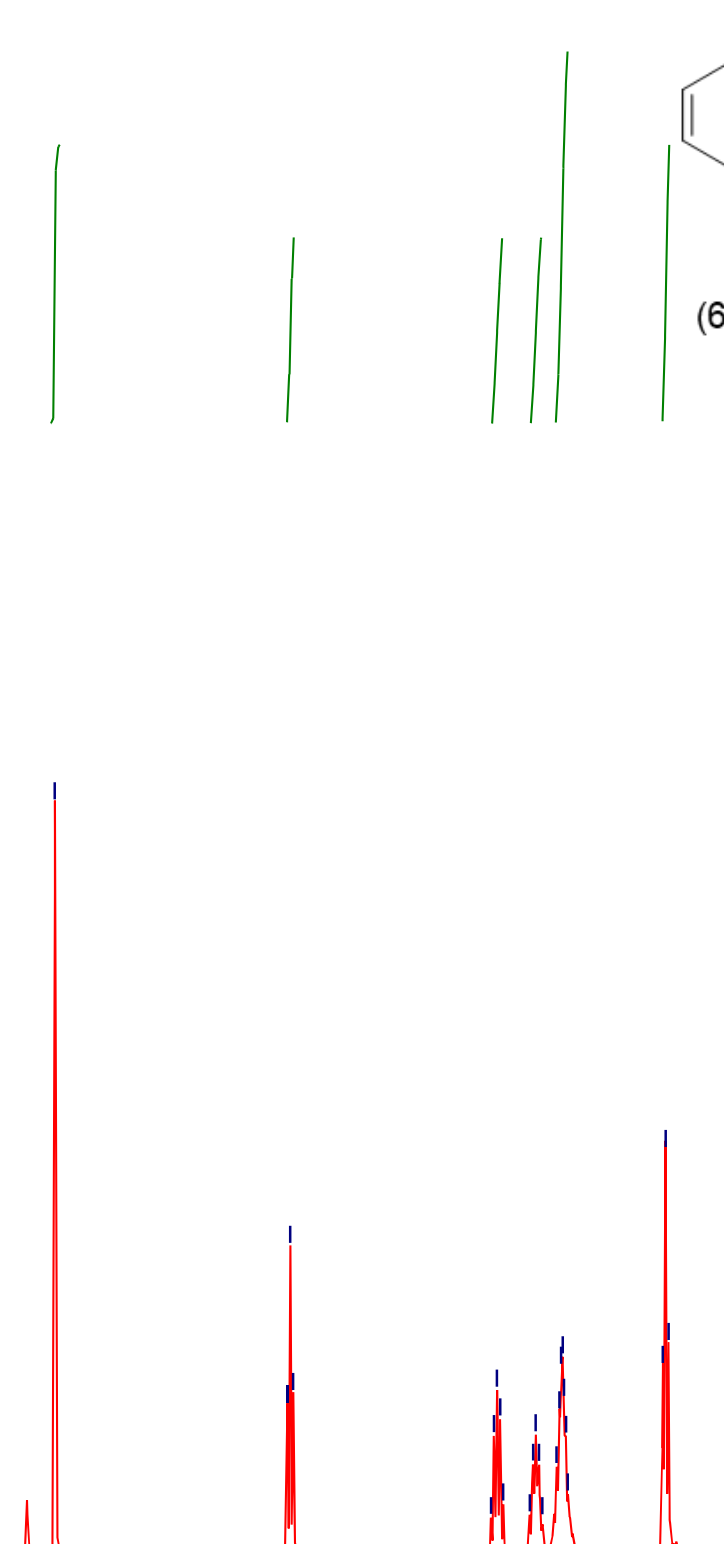

4145
8808 
1h; ${ }^{13} \mathrm{C}$ NMR

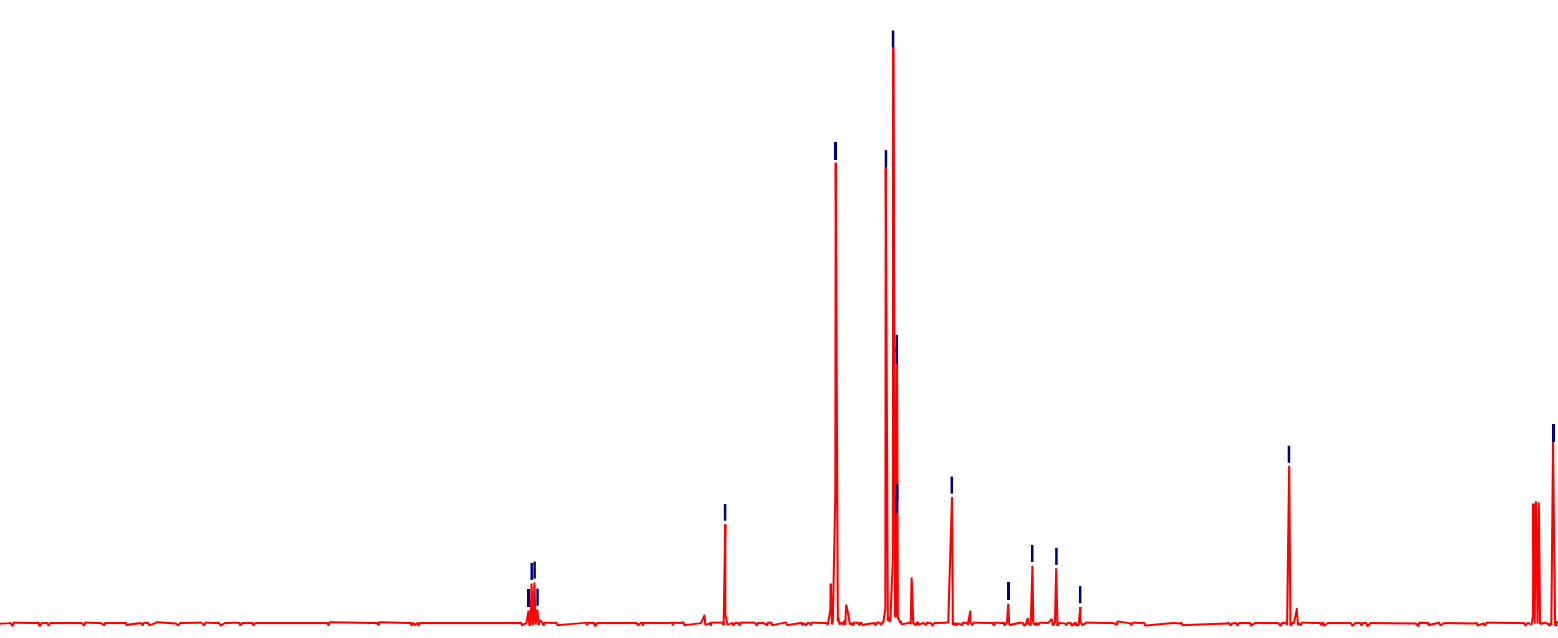



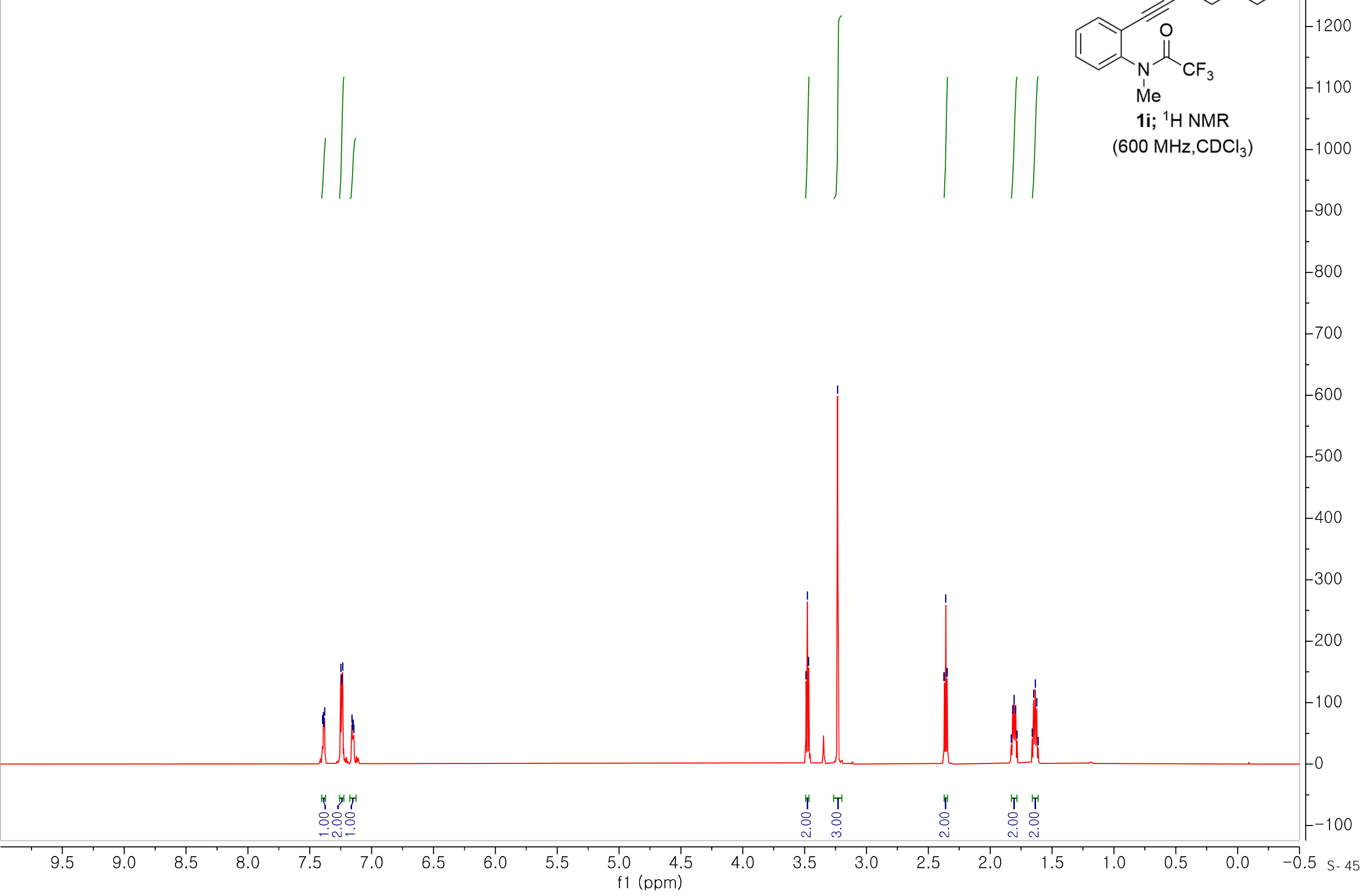

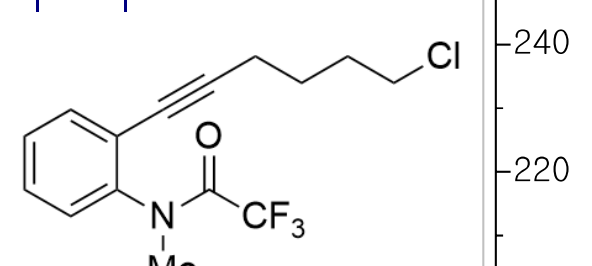

$\mathrm{Me}$

1i; ${ }^{13} \mathrm{C}$ NMR

(151 MHz, $\mathrm{CDCl}_{3}$ )

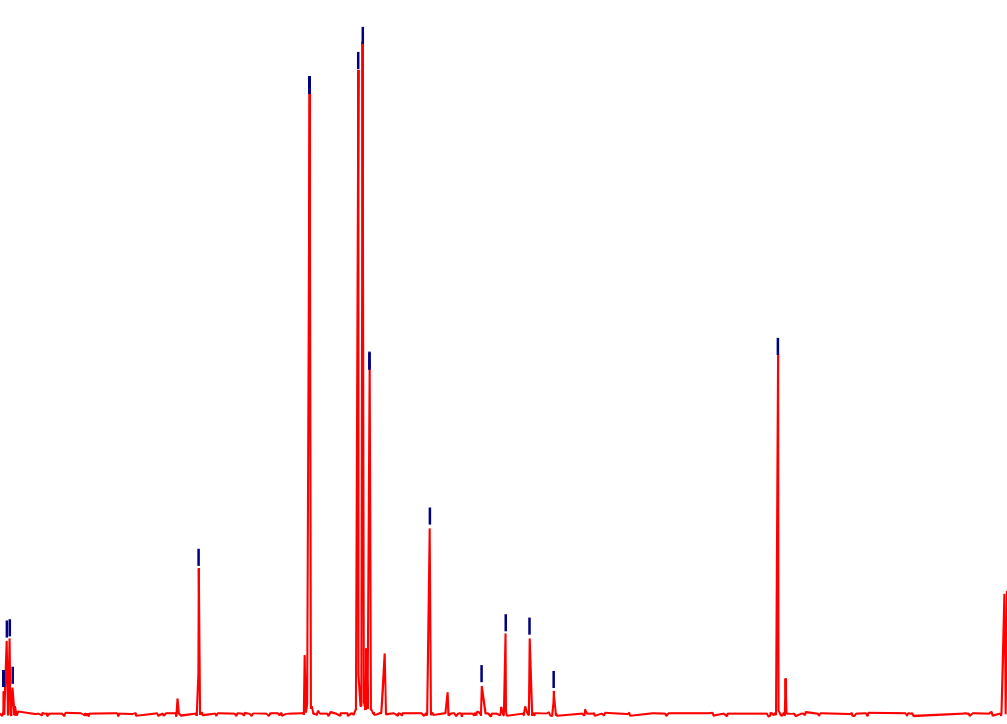




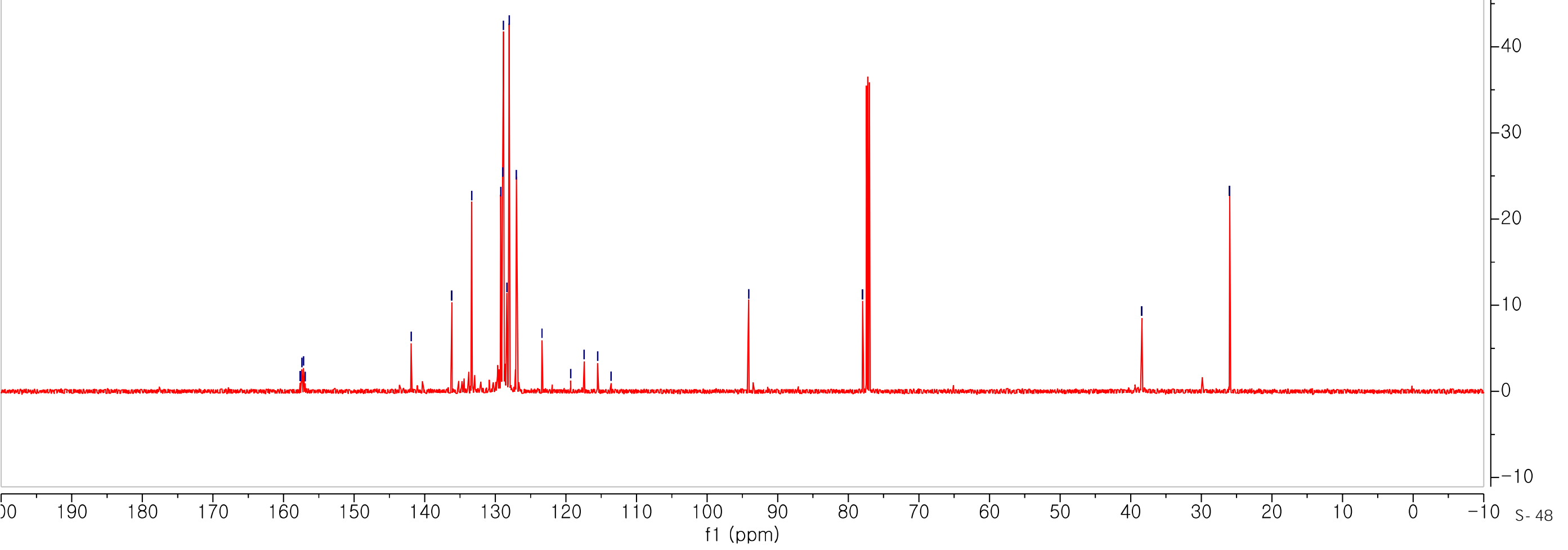




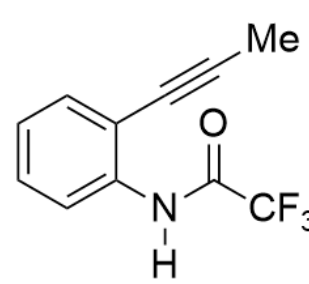

1k; ${ }^{13} \mathrm{C}$ NMR $\left(151 \mathrm{MHz} \mathrm{CDCl}_{3}\right.$ )

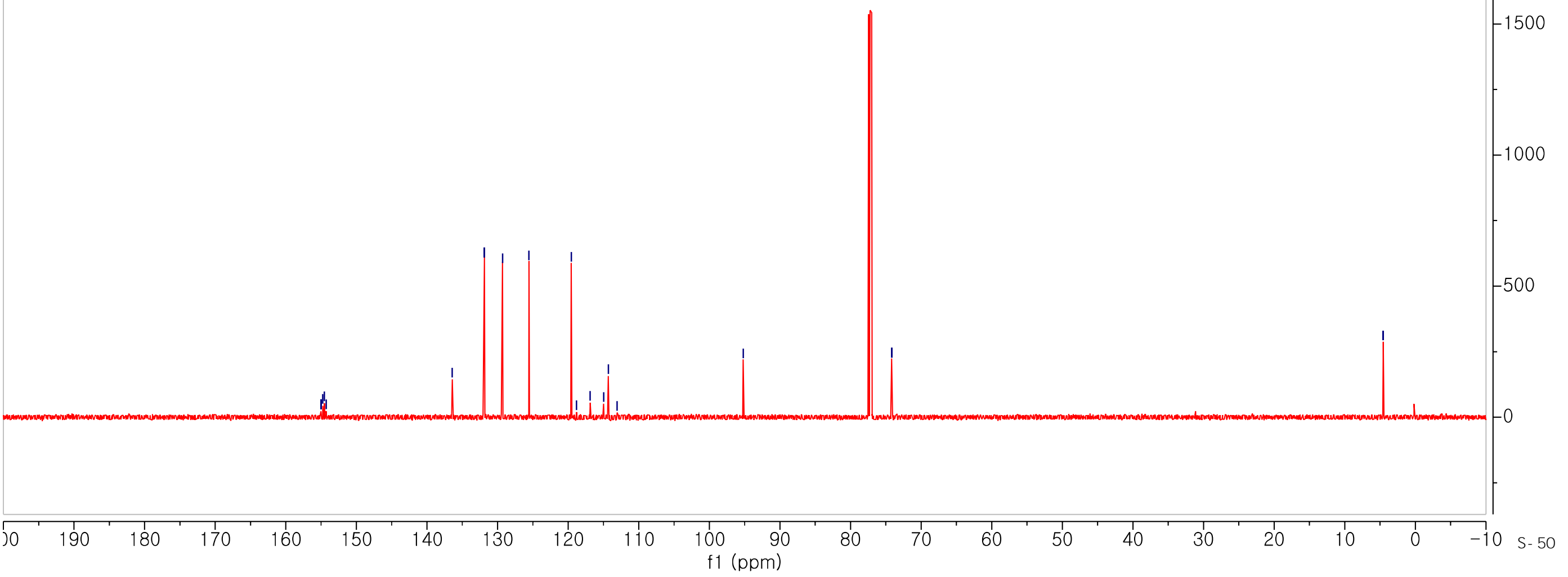




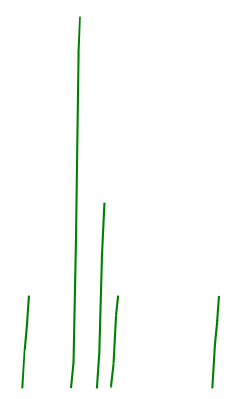

1I; ${ }^{1} \mathrm{H}$ NMR (600 $\mathrm{MHz}, \mathrm{CDCl}_{3}$ )
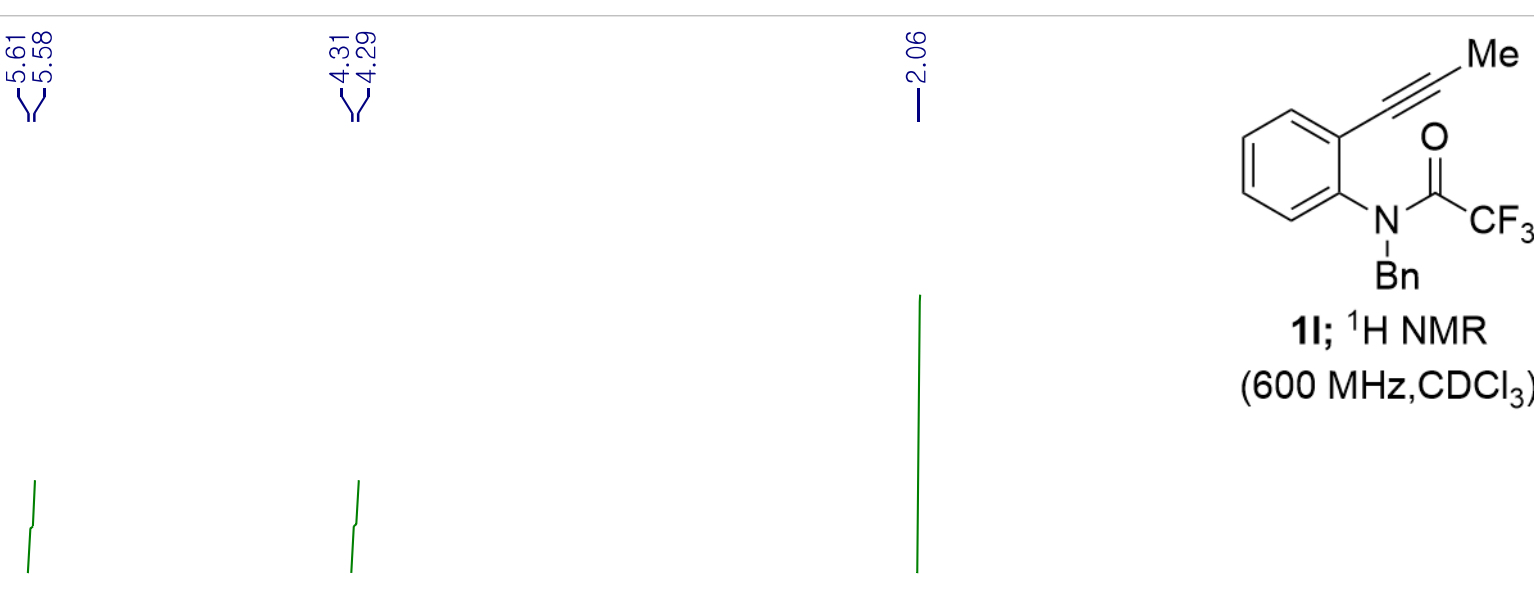

$\mathrm{Bn}$

$-900$

$-800$

$-700$

$-600$

$-500$

$-400$

$-300$

$-200$

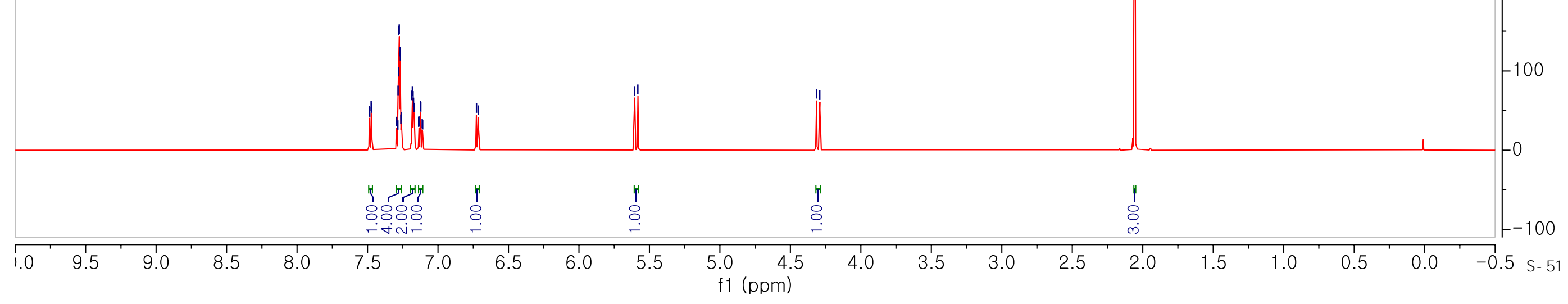



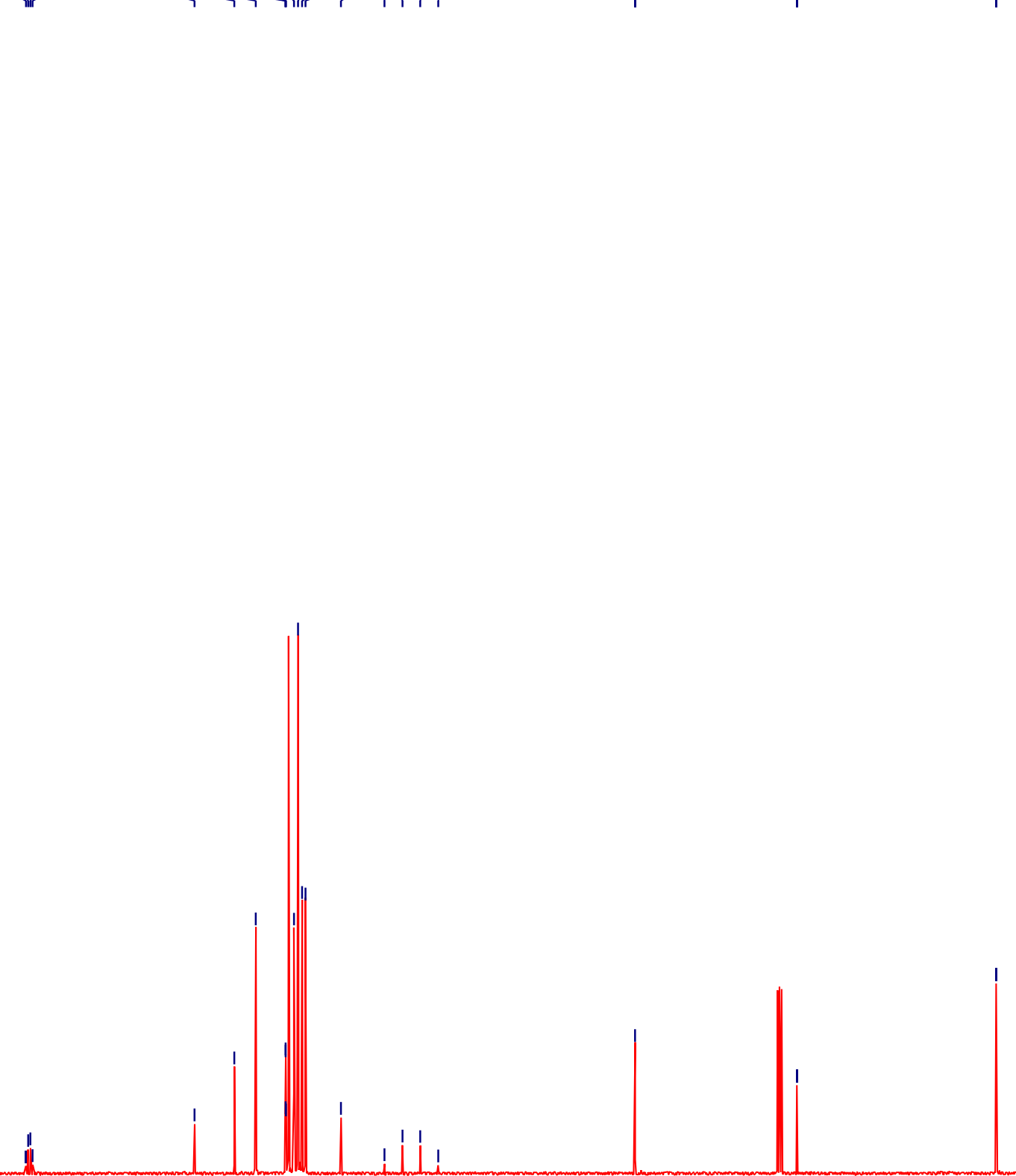

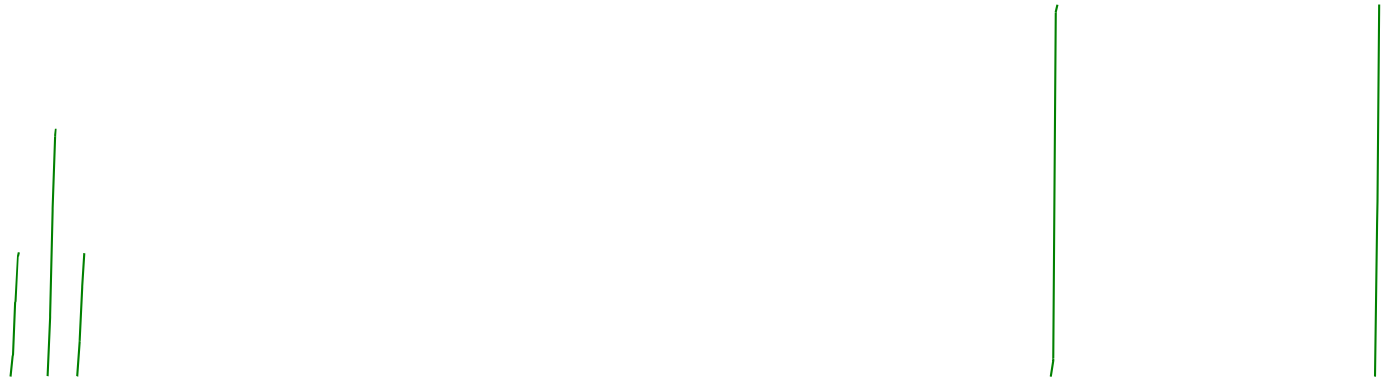

1m; ${ }^{1} \mathrm{H}$ NMR 


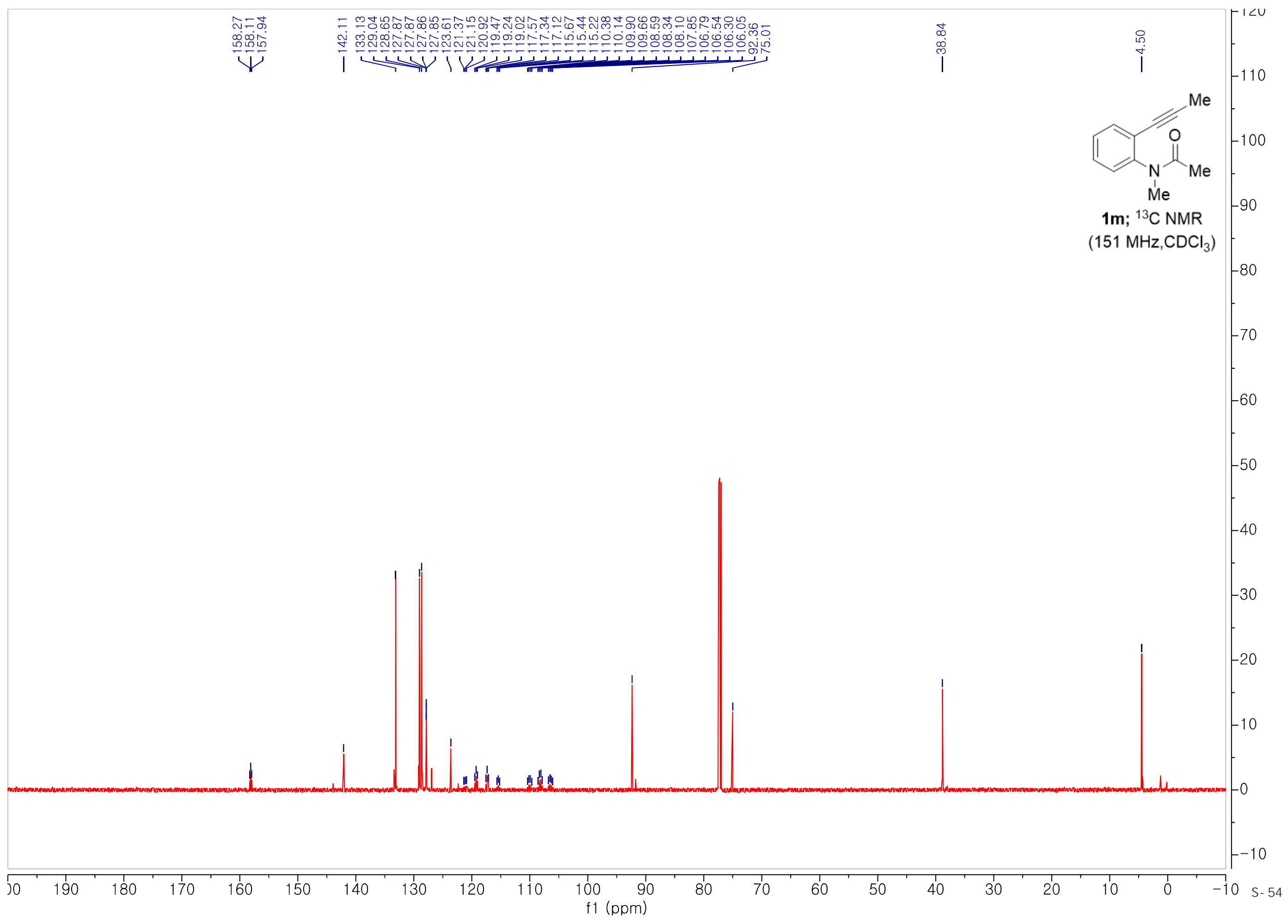




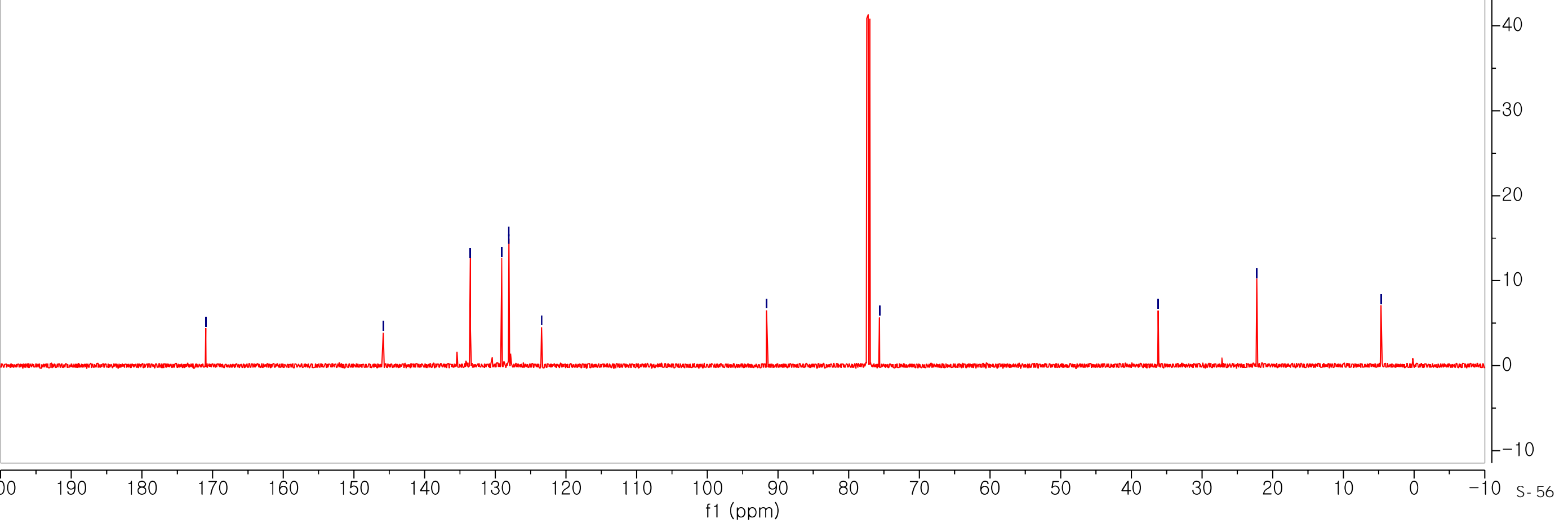


10; ${ }^{13} \mathrm{C}$ NMR 


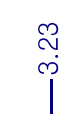

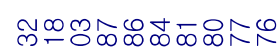

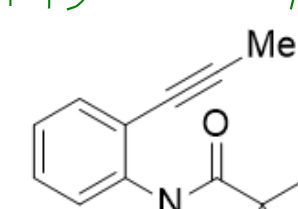

$\mathrm{Me}$ ind $\mathrm{Me}$

1p; ${ }^{1} \mathrm{H}$ NMR

$\left(600 \mathrm{MHz}, \mathrm{CDCl}_{3}\right)$

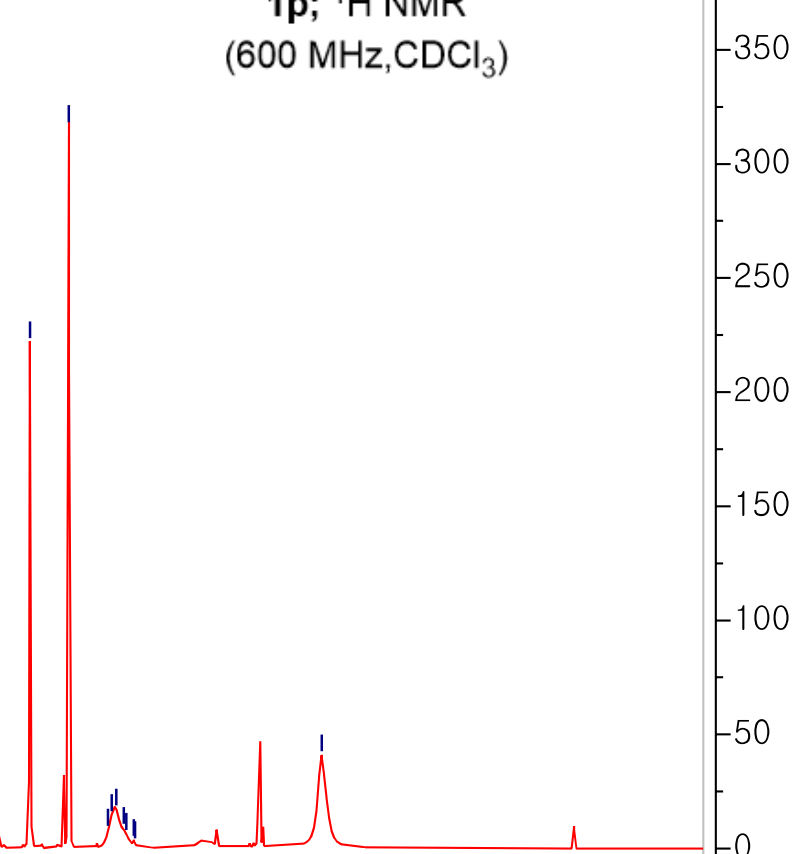

14
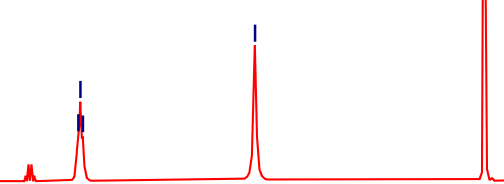

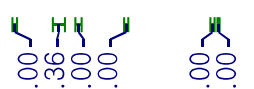

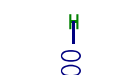

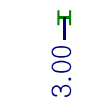




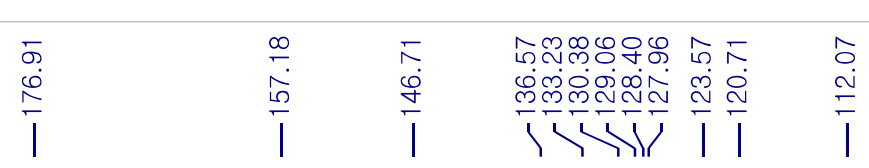

(25 


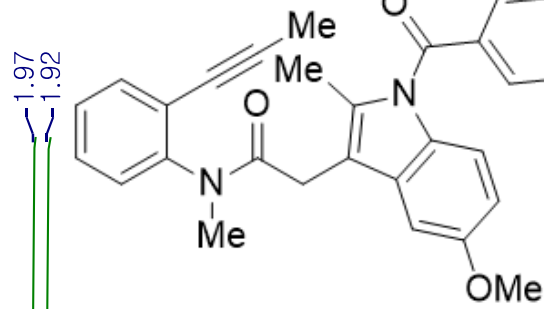

1q; ${ }^{1} \mathrm{H}$ NMR $\left(600 \mathrm{MHz}, \mathrm{CDCl}_{3}\right)$

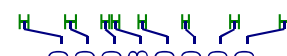
80800808

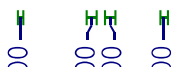




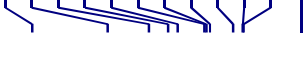

1/1

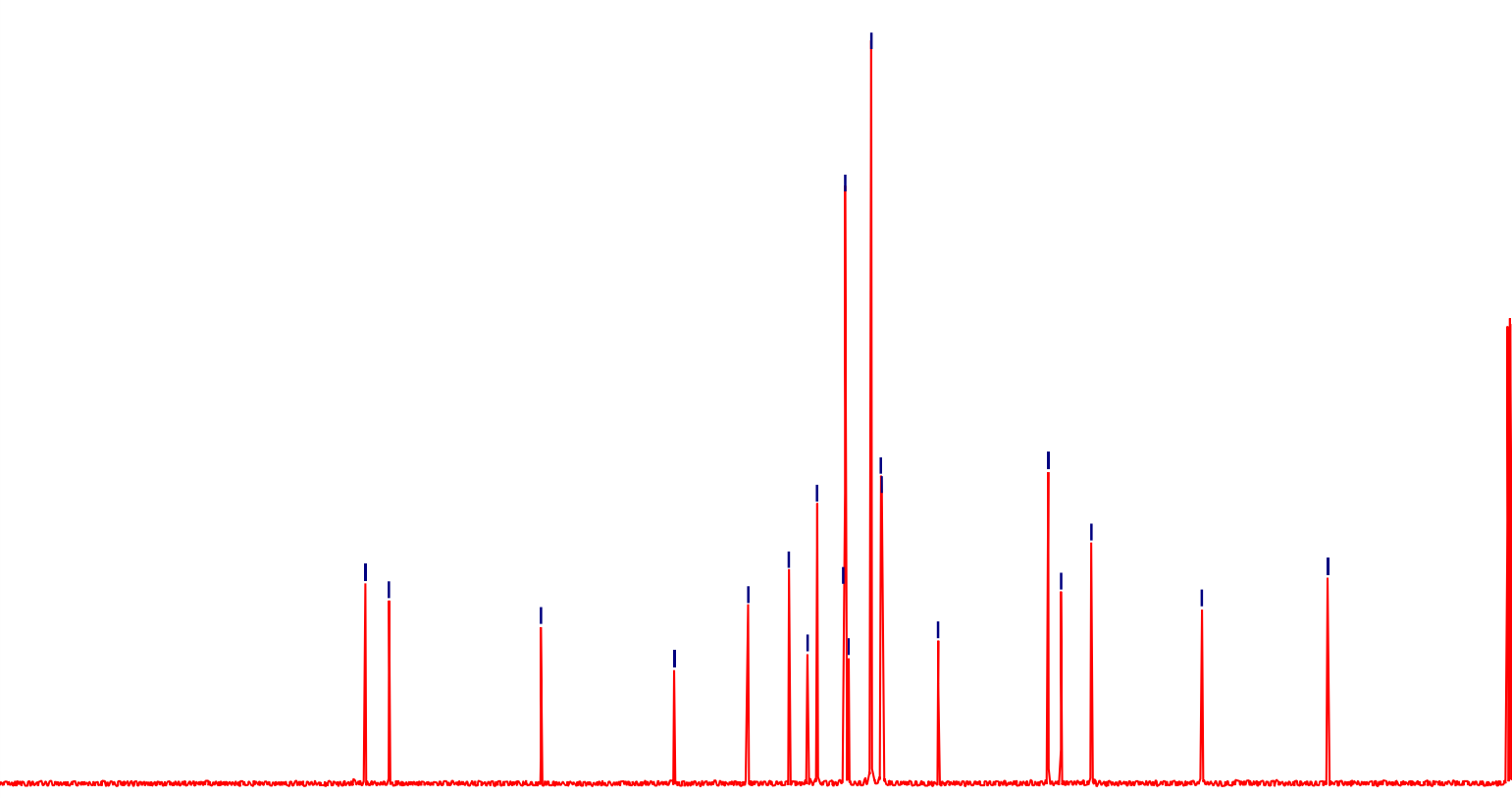

1q; ${ }^{13} \mathrm{C}$ NMR

(151 $\mathrm{MHz}, \mathrm{CDCl}_{3}$ )

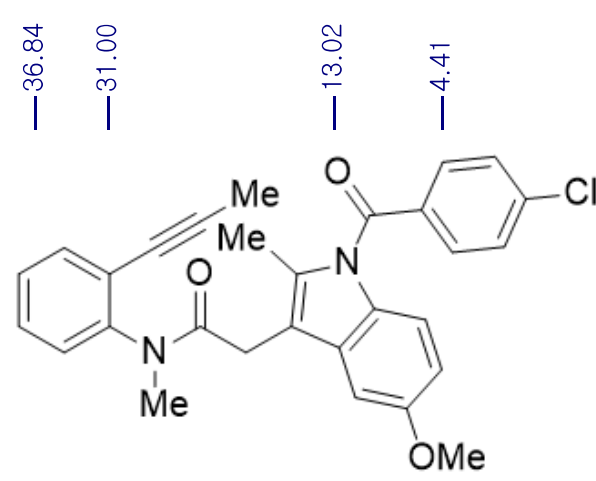



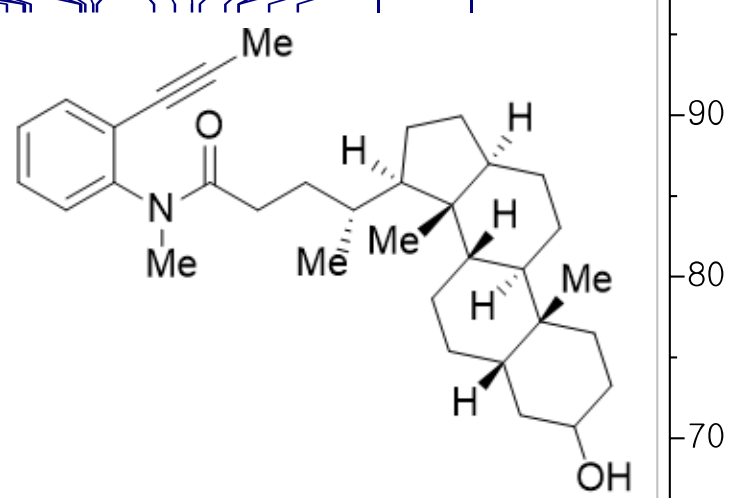

1r; ${ }^{13} \mathrm{C}$ NMR

(151 $\mathrm{MHz}, \mathrm{CDCl}_{3}$ )

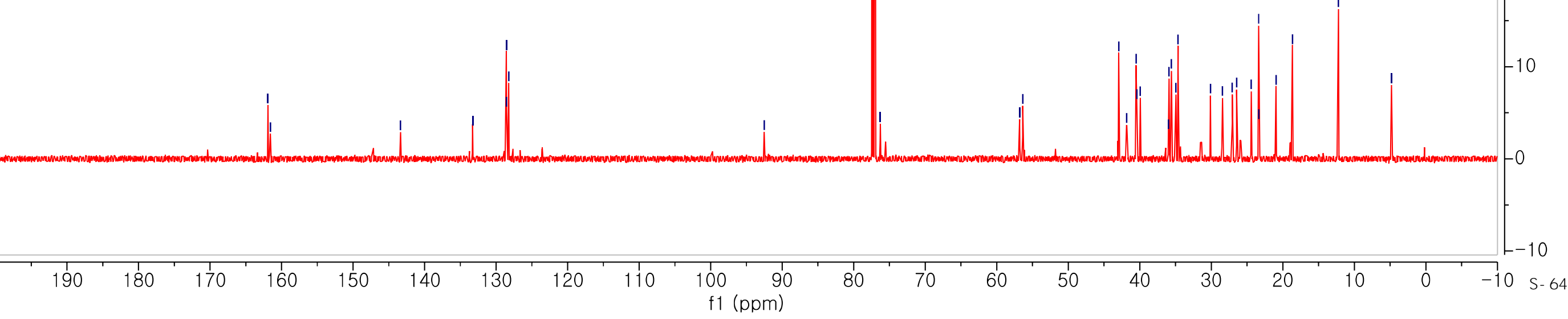



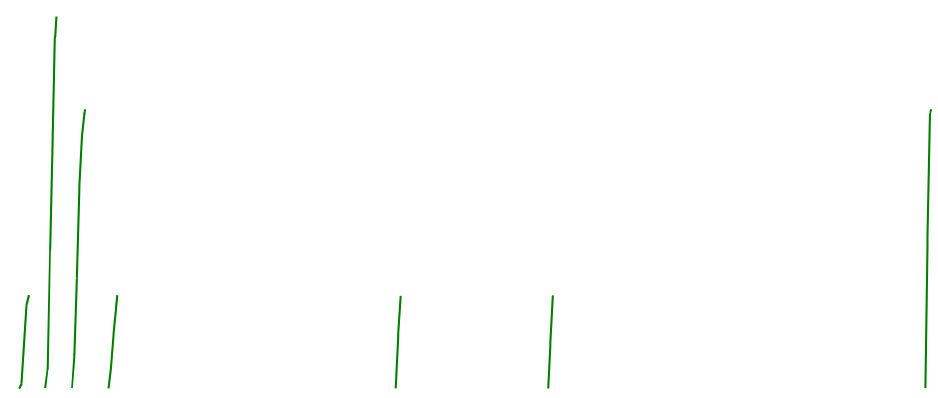

\section{3aa; ${ }^{1} \mathrm{H}$ NMR} (600 $\mathrm{MHz}, \mathrm{CDCl}_{3}$ )

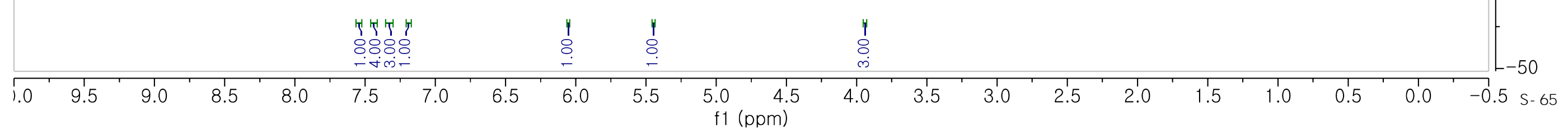




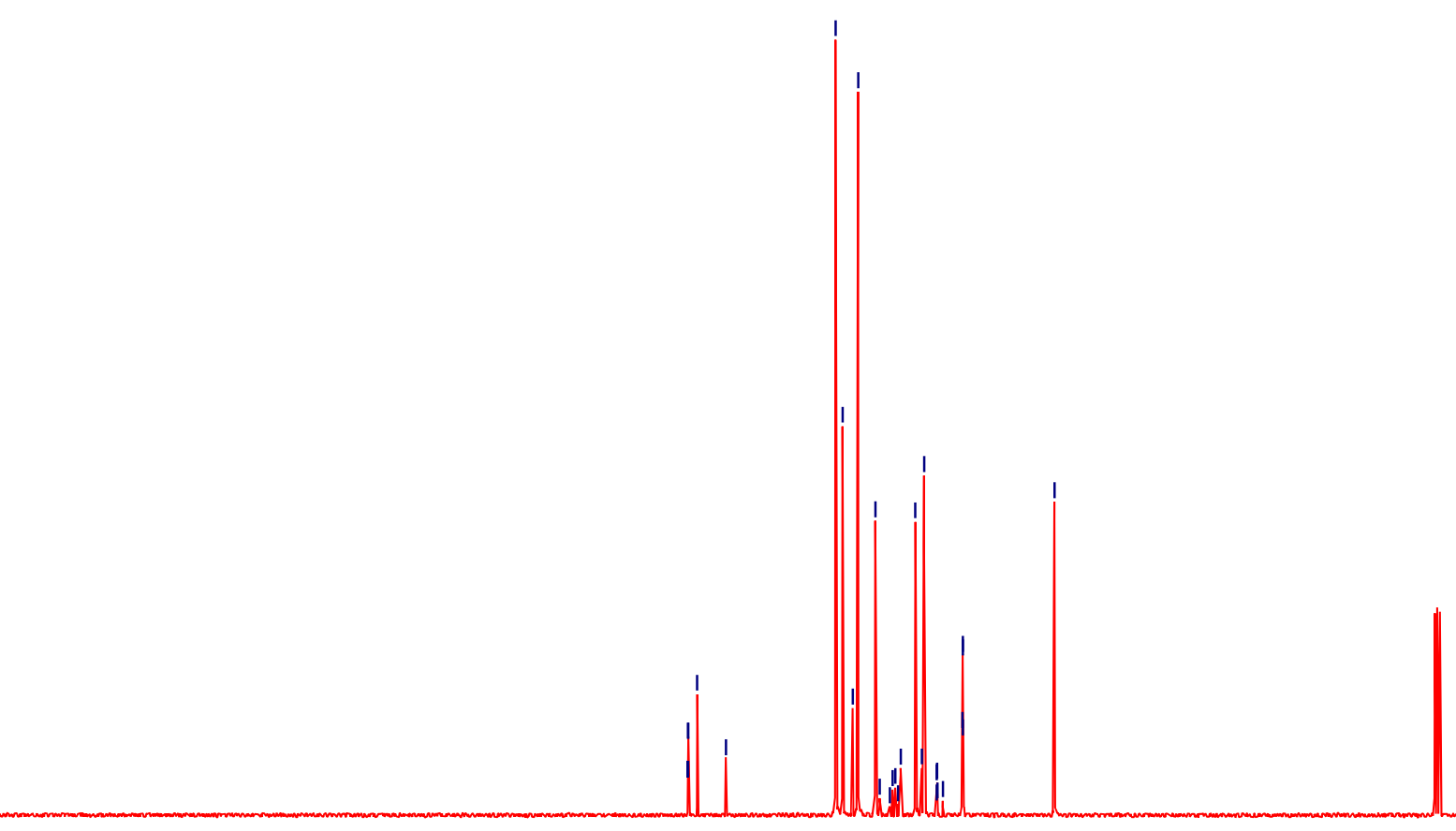



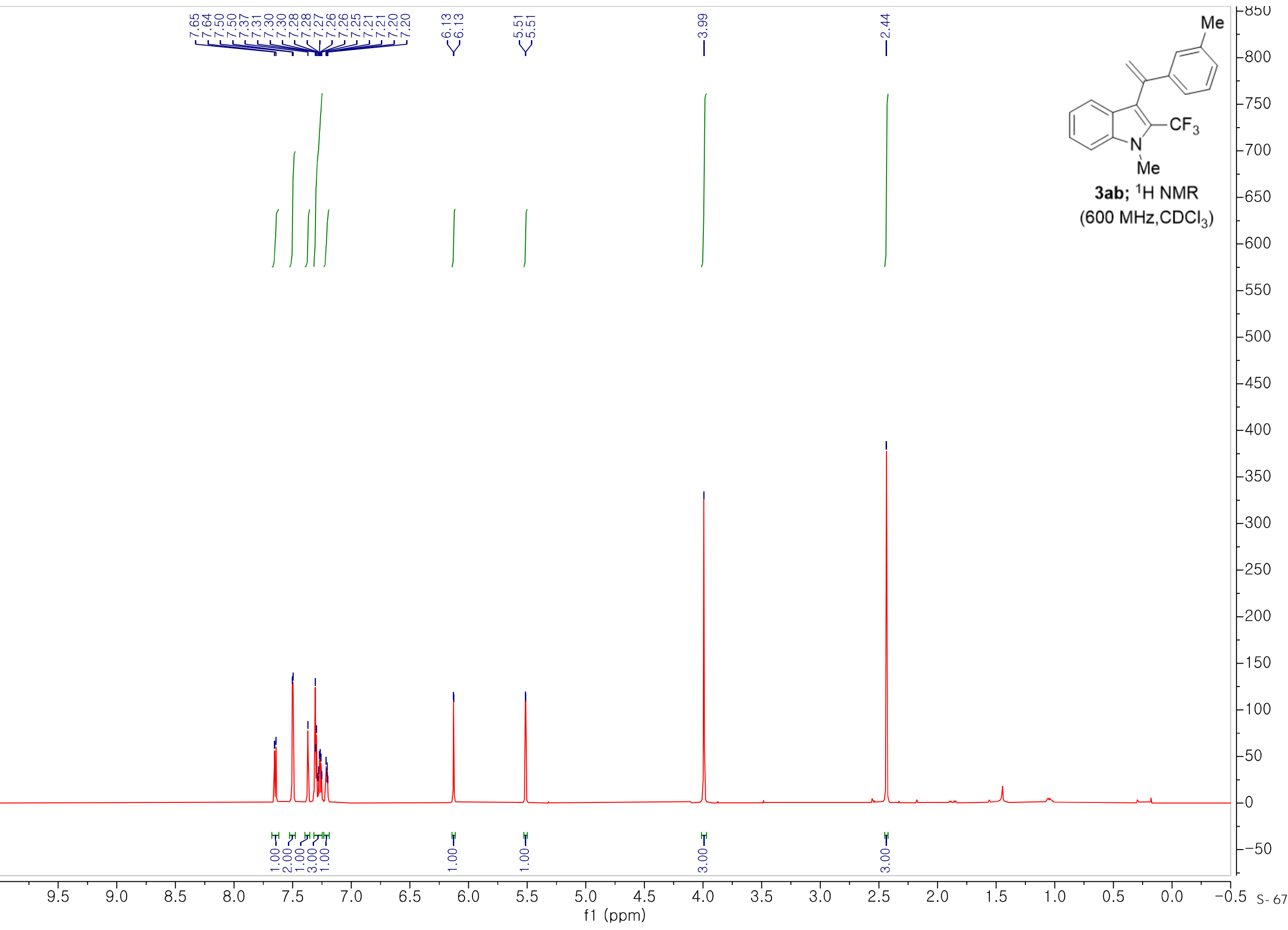


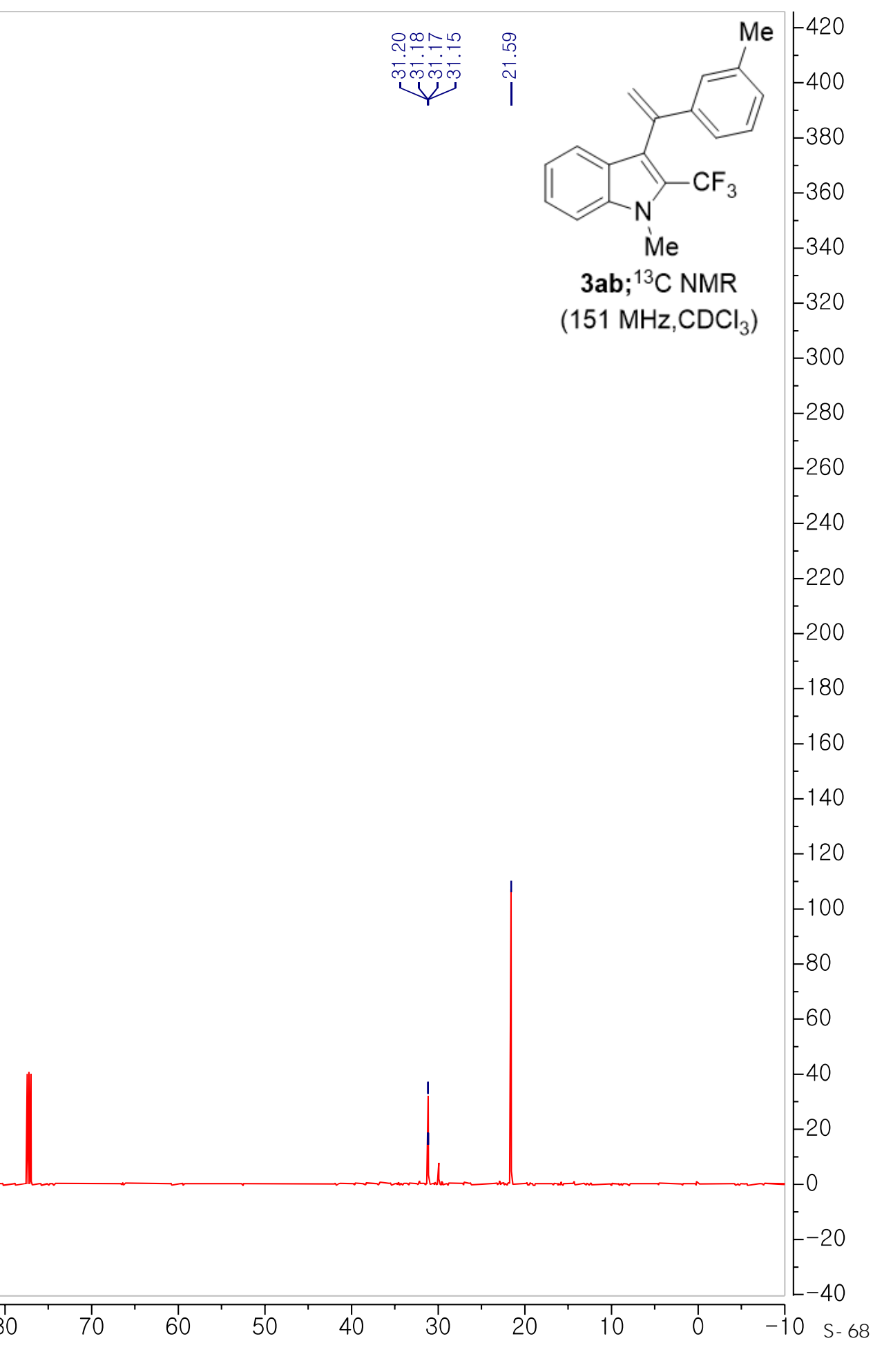




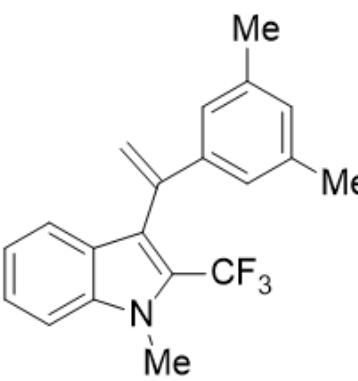

$\mathrm{Me}$

3ac; ${ }^{1} \mathrm{H}$ NMR (600 $\mathrm{MHz}, \mathrm{CDCl}_{3}$ )

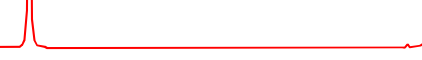



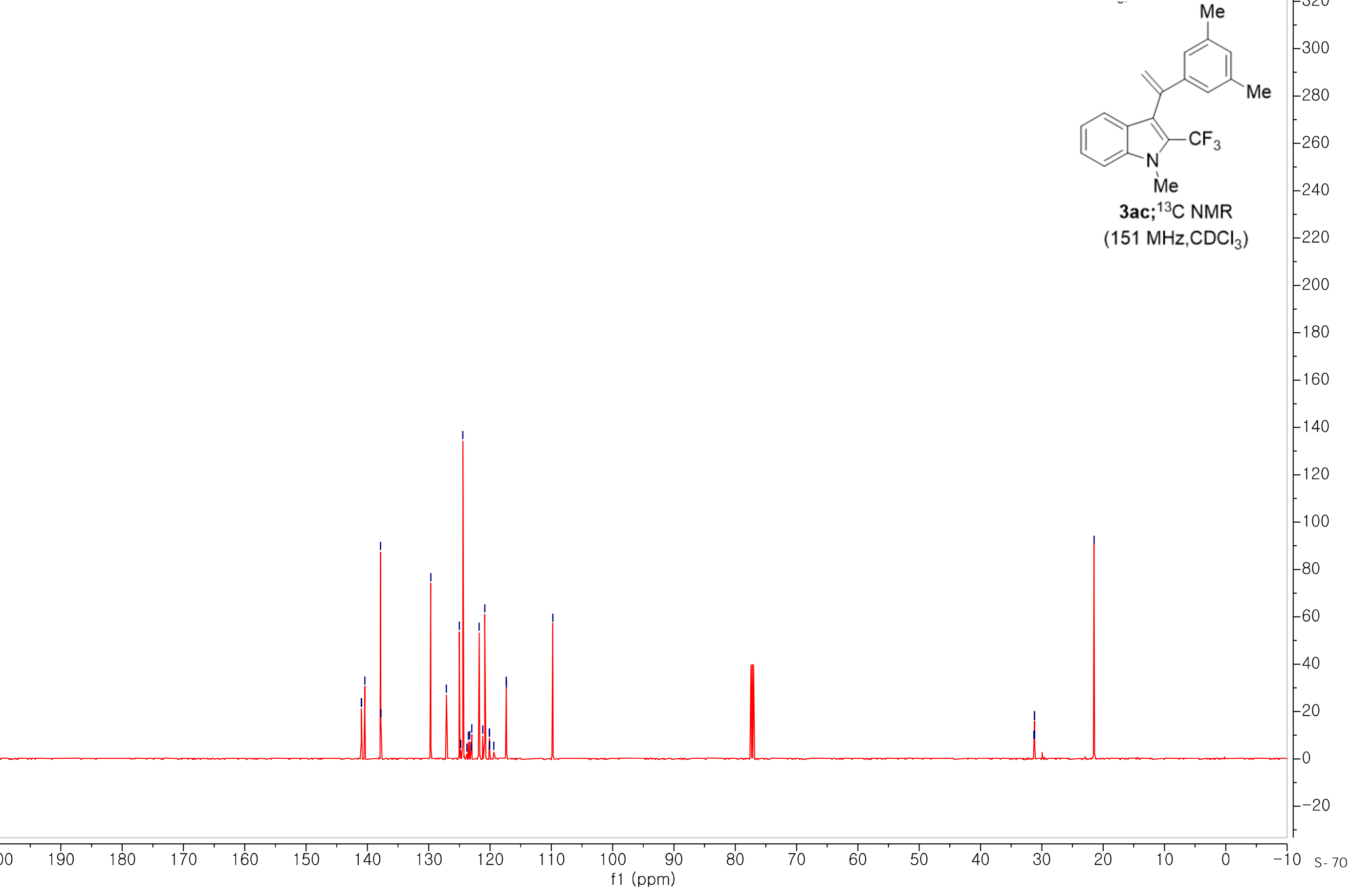
| || |

3ad; ${ }^{1} \mathrm{H}$ NMR

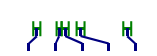

88888 


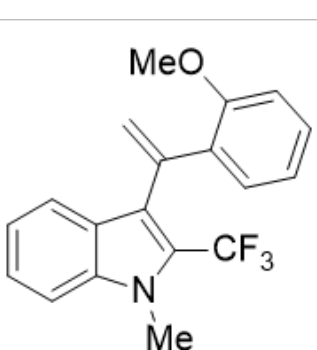

3ae; ${ }^{1} \mathrm{H}$ NMR $\left(600 \mathrm{MHz}, \mathrm{CDCl}_{3}\right)$

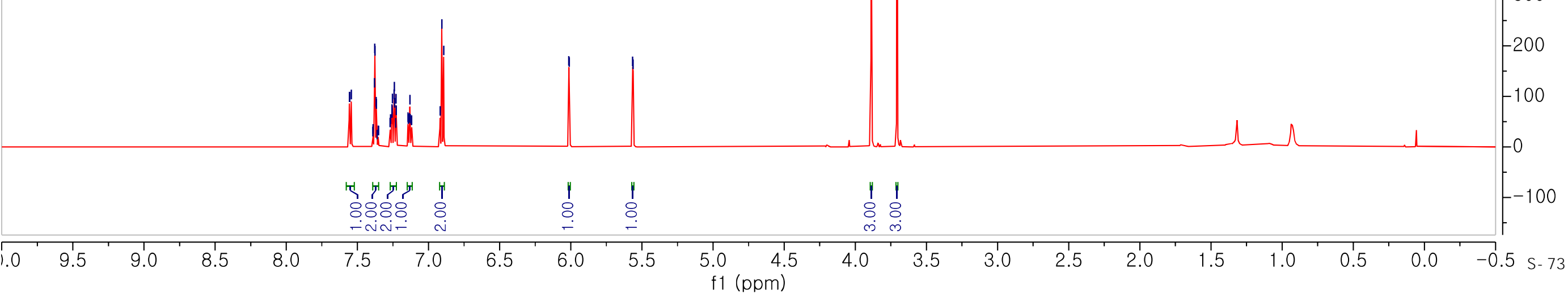



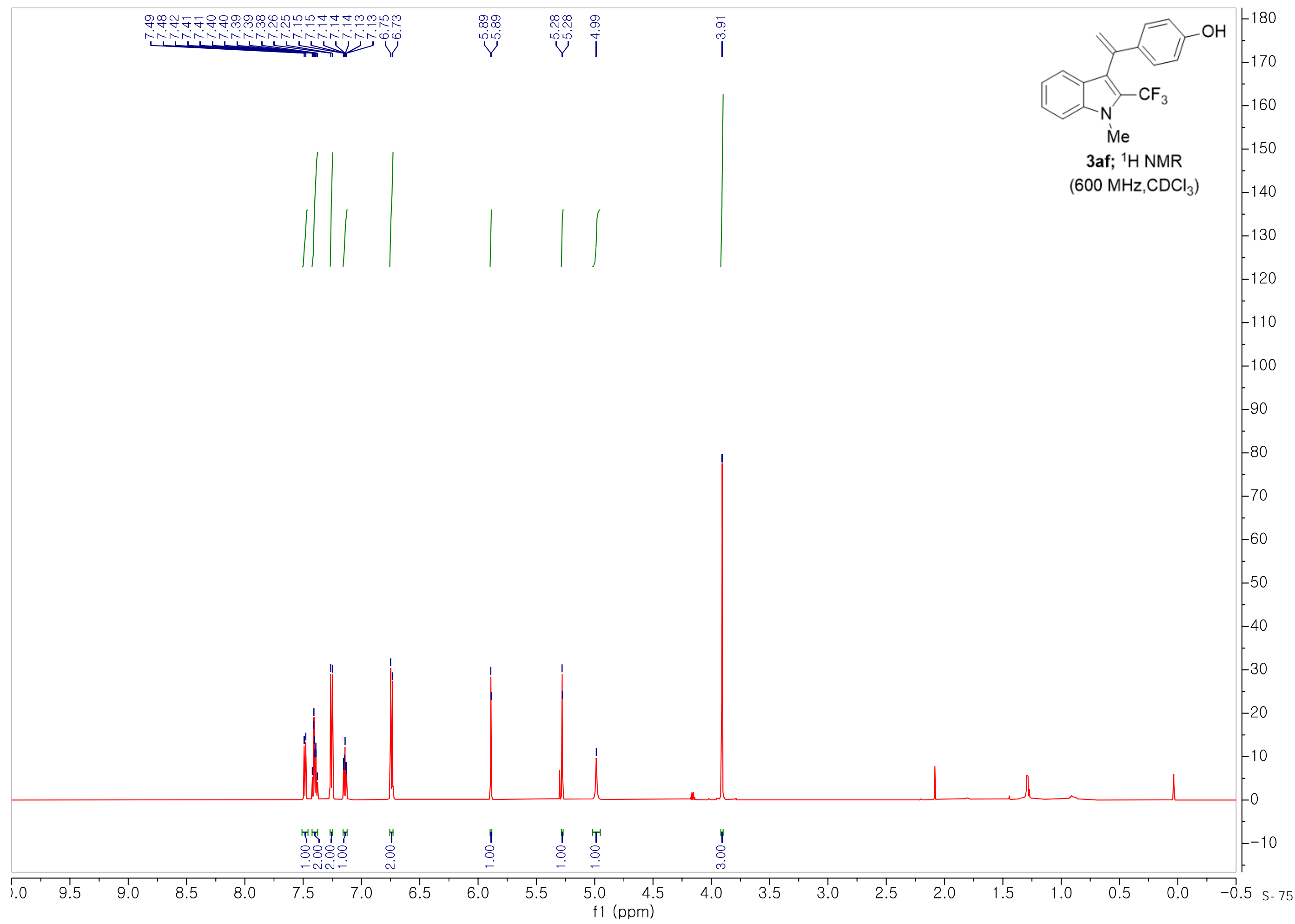


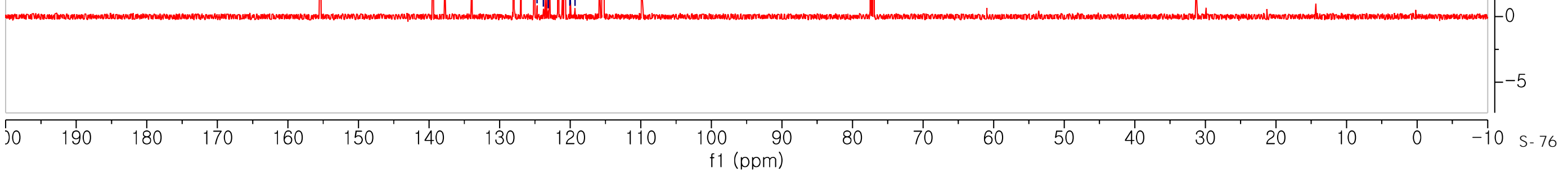



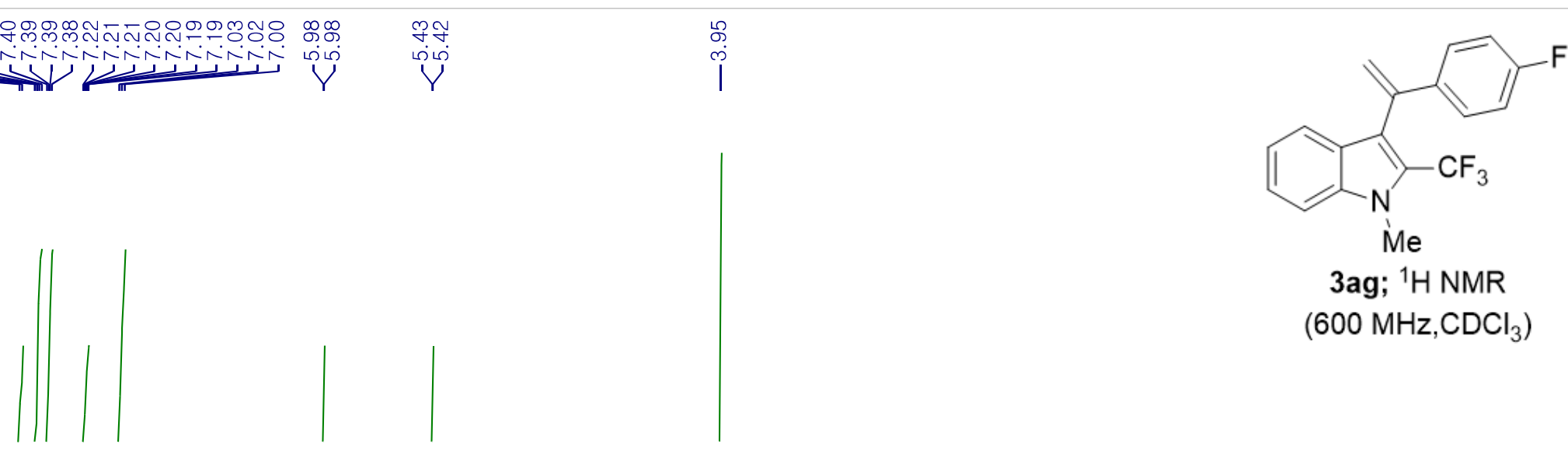

$-2000$

3ag; ${ }^{1} \mathrm{H}$ NMR $-1600$ $\left(600 \mathrm{MHz}, \mathrm{CDCl}_{3}\right)$ 


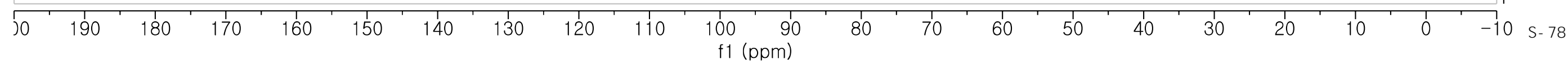



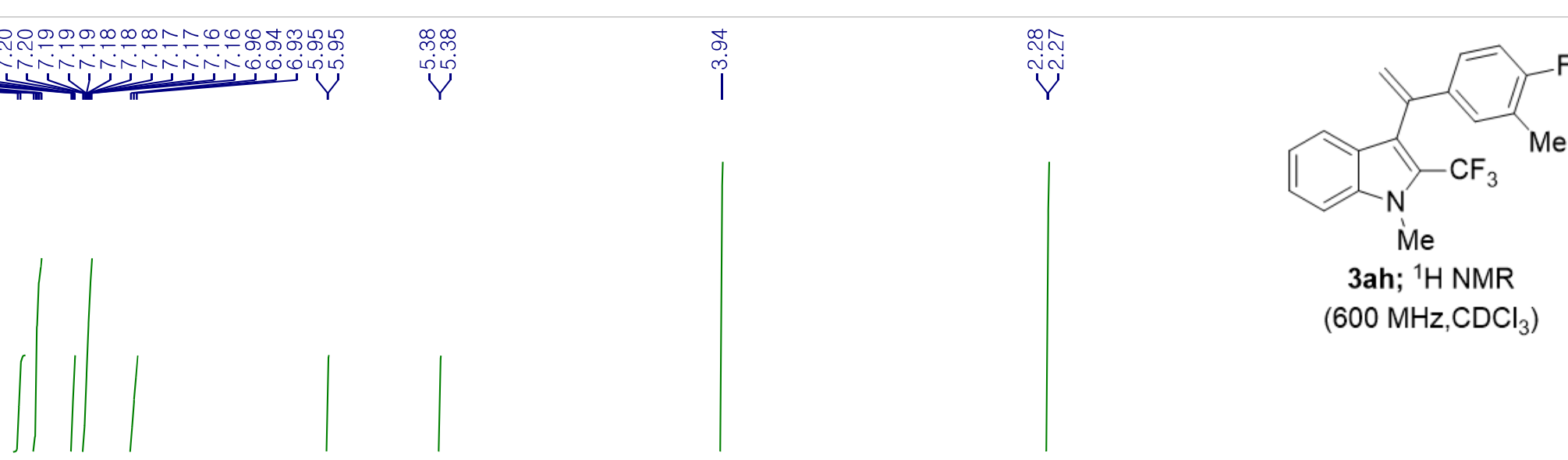

Me

3ah; ${ }^{1} \mathrm{H}$ NMR $\left(600 \mathrm{MHz}, \mathrm{CDCl}_{3}\right.$ ) 


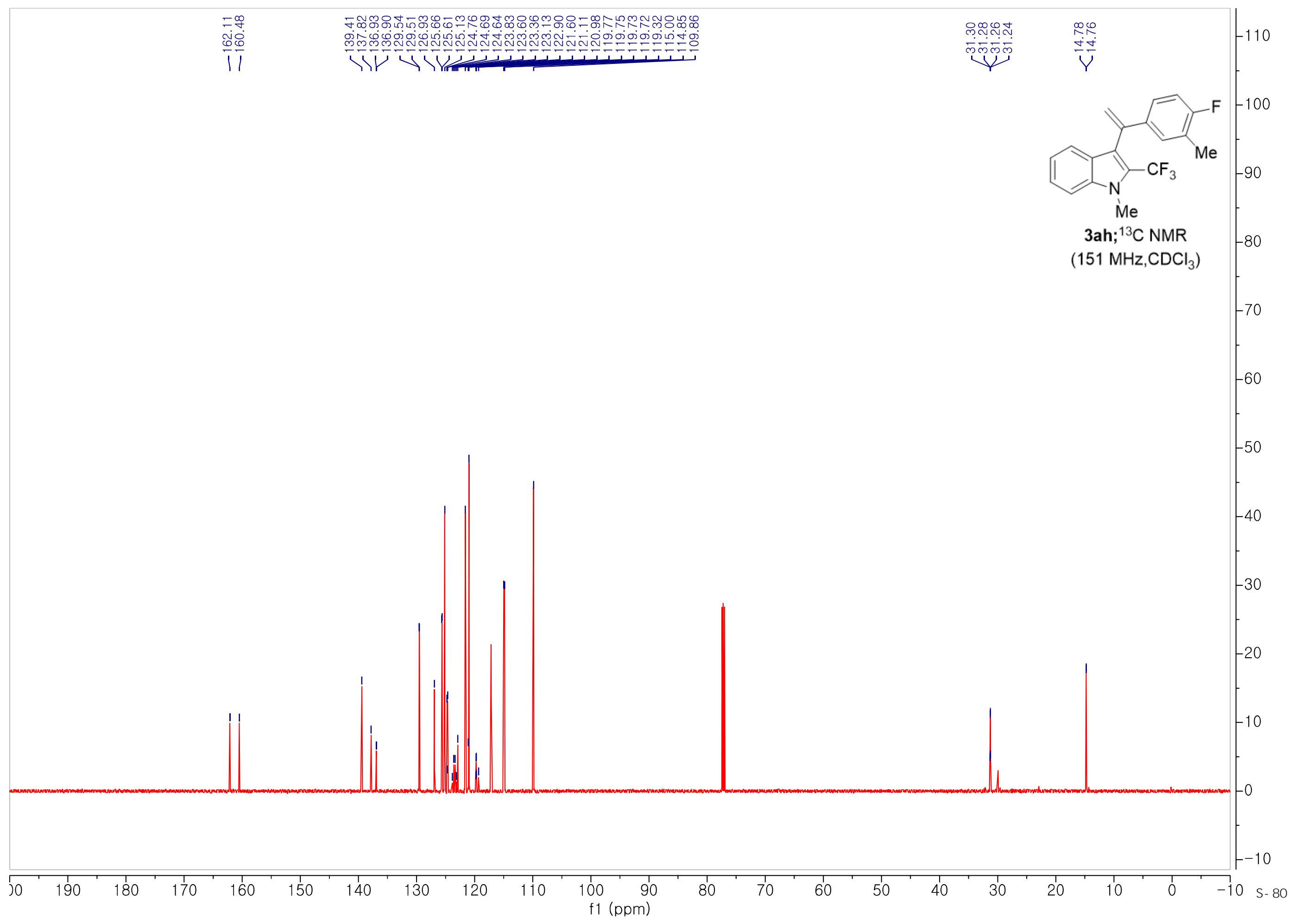




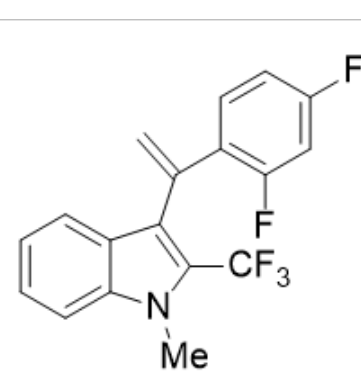

$-380$

3ai; ${ }^{1} \mathrm{H}$ NMR

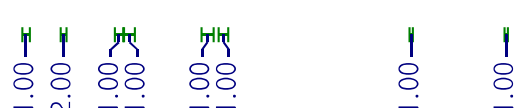



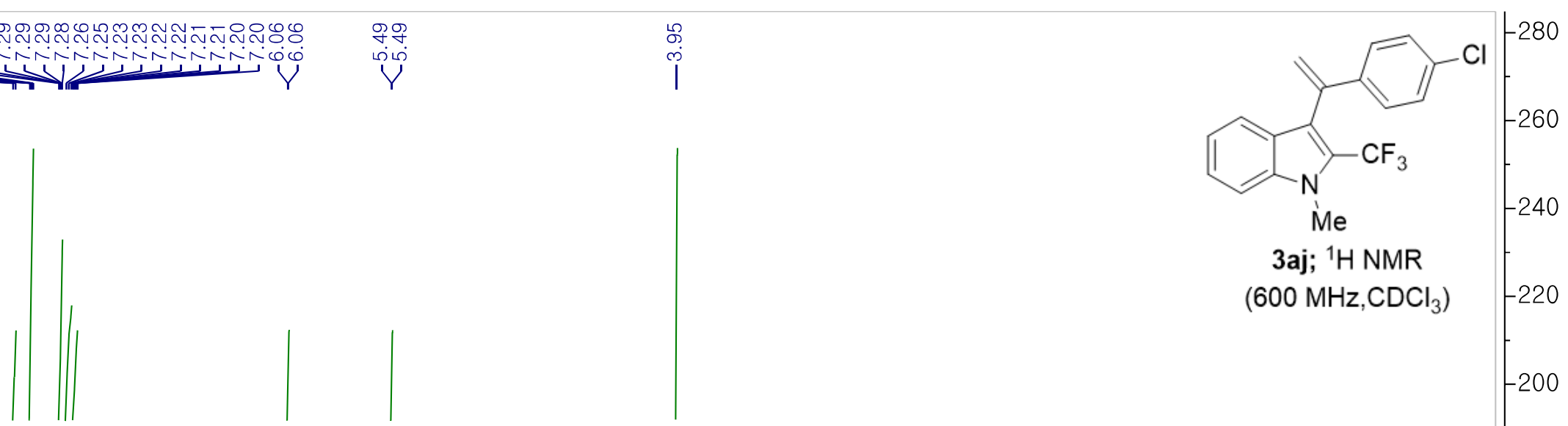

3aj; ${ }^{1} \mathrm{H}$ NMR $\left(600 \mathrm{MHz}, \mathrm{CDCl}_{3}\right)$

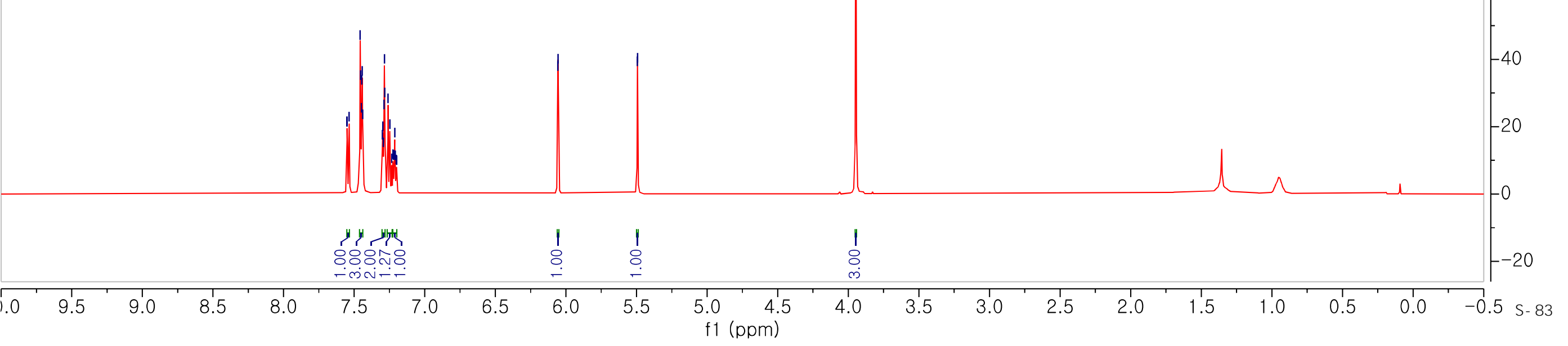




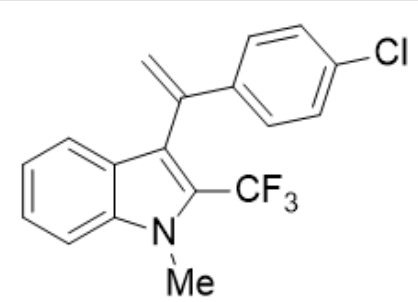

$\mathrm{Me}$

3aj; ${ }^{13} \mathrm{C}$ NMR $\left(151 \mathrm{MHz}, \mathrm{CDCl}_{3}\right.$ ) 

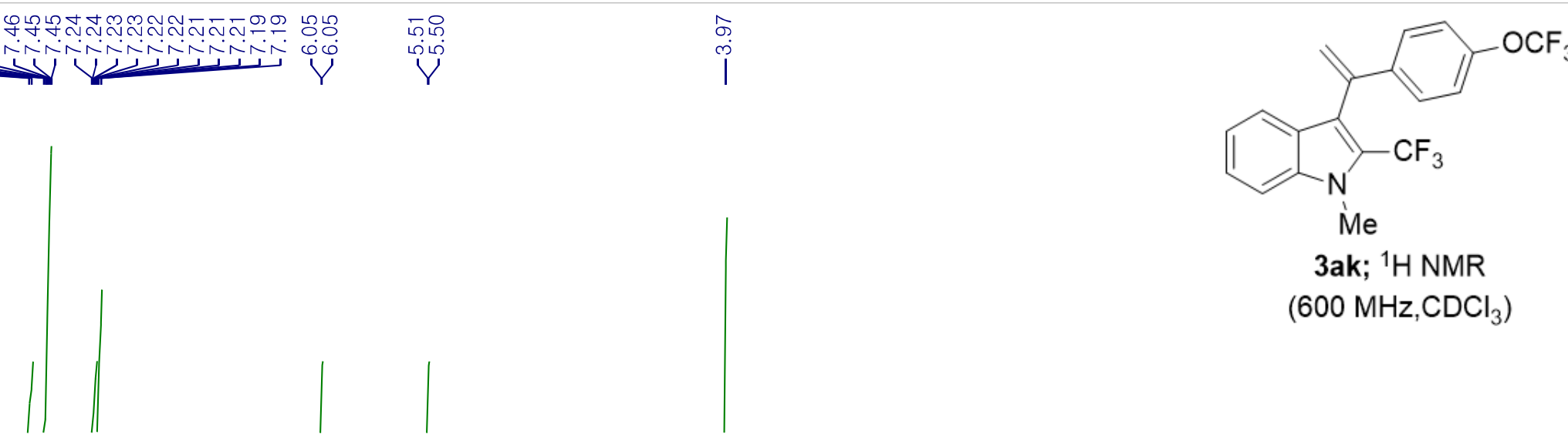

$-2600$

3ak; ${ }^{1} \mathrm{H}$ NMR

$\left(600 \mathrm{MHz}, \mathrm{CDCl}_{3}\right)$ 


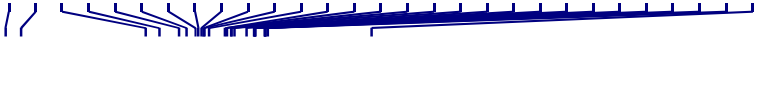

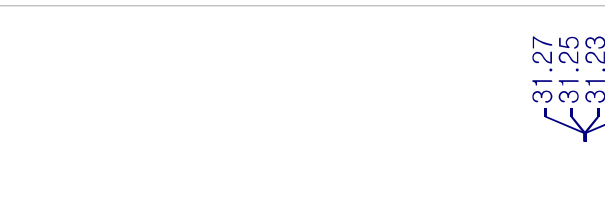

3ak; ${ }^{13} \mathrm{C}$ NMR 

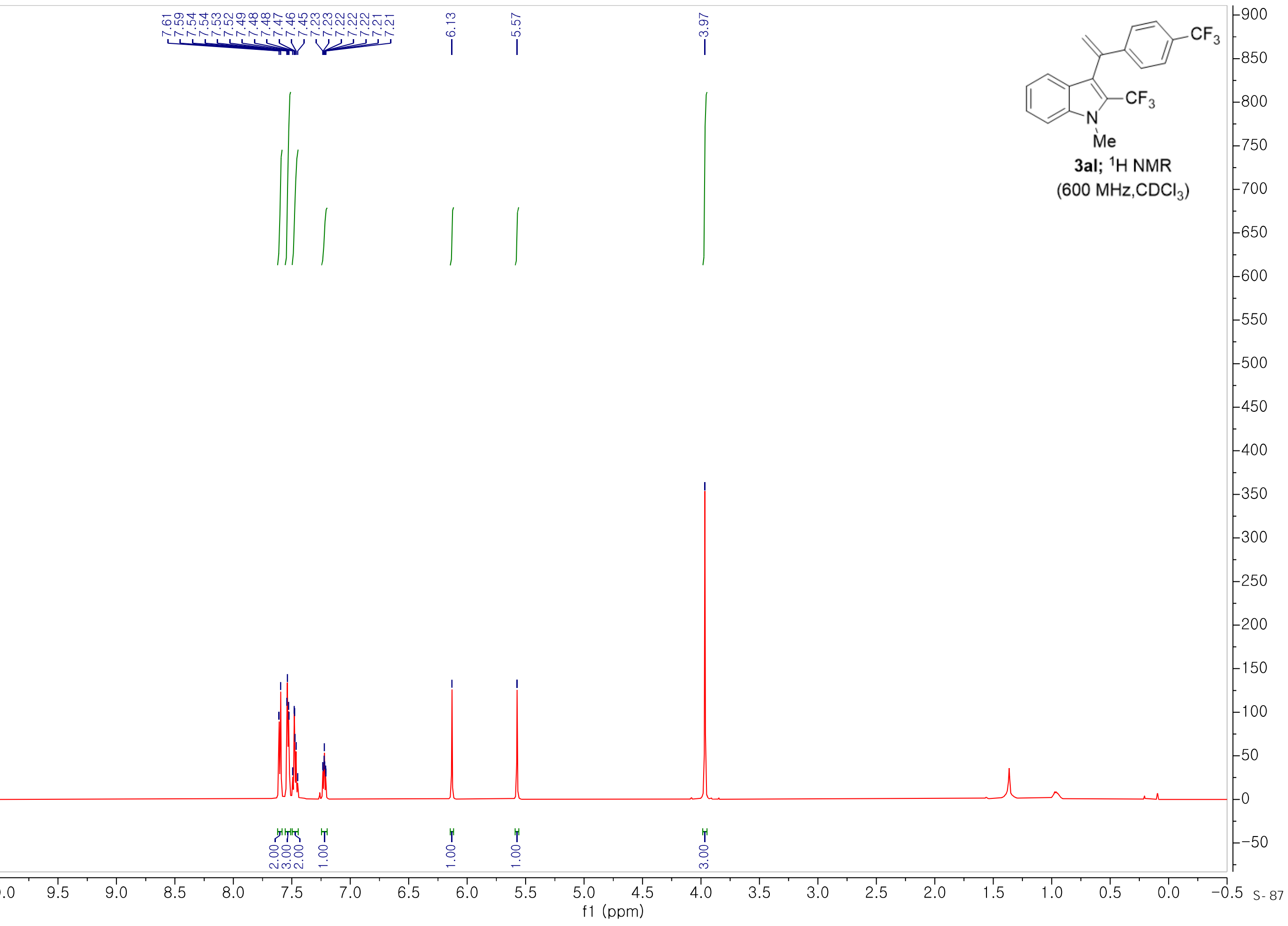


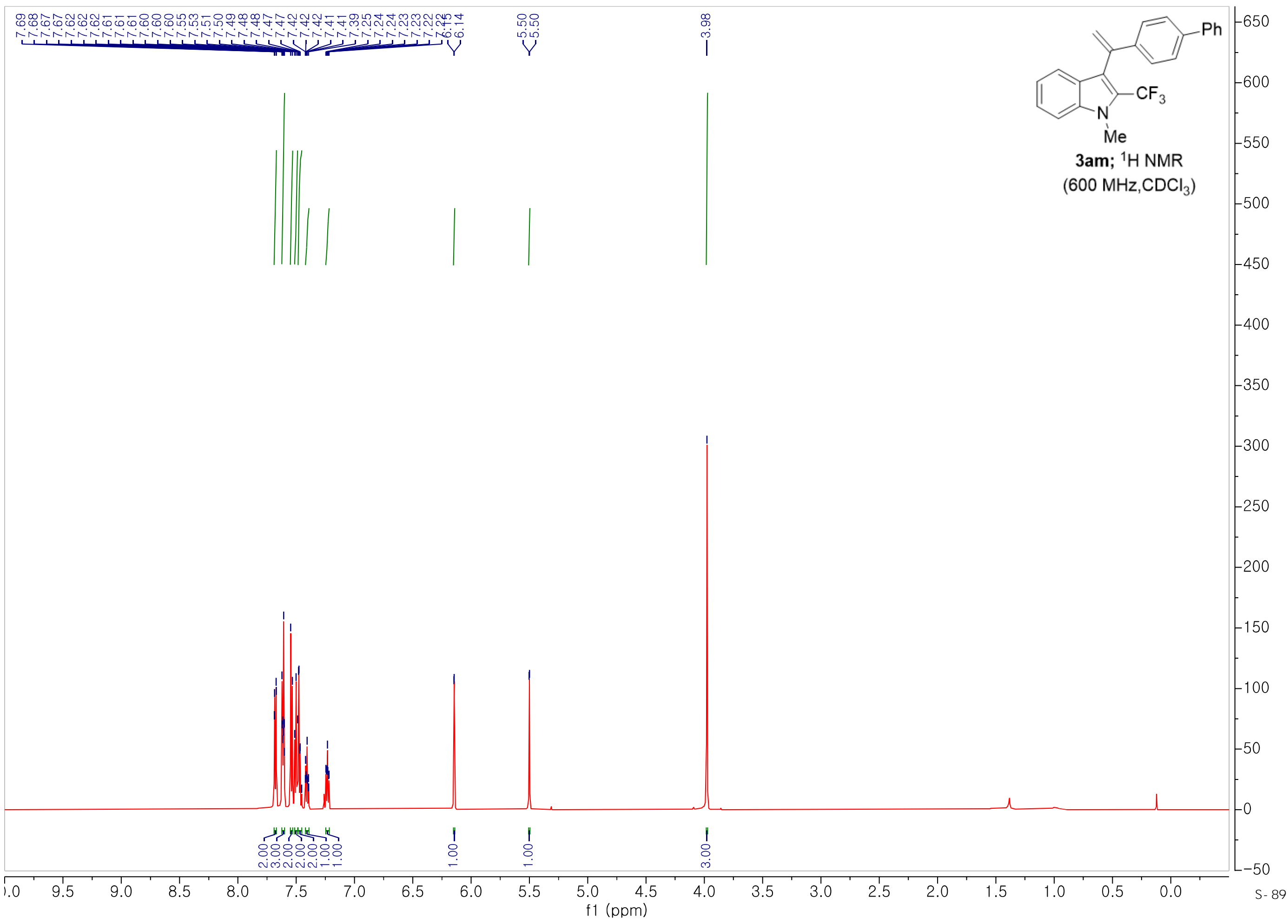



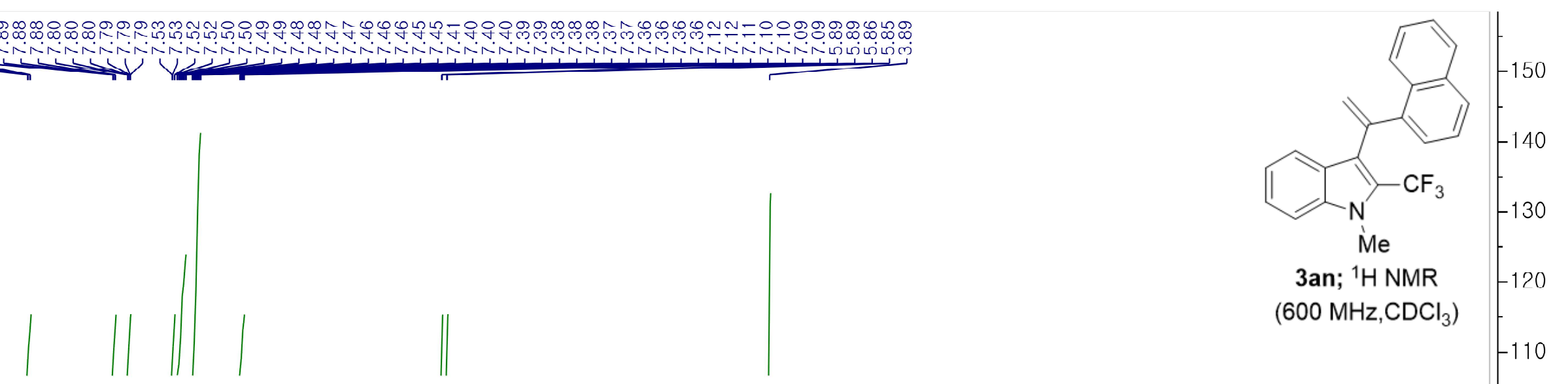

3an; ${ }^{1} \mathrm{H}$ NMR

$\left(600 \mathrm{MHz}^{-\mathrm{CDCl}_{3}}\right)$ 

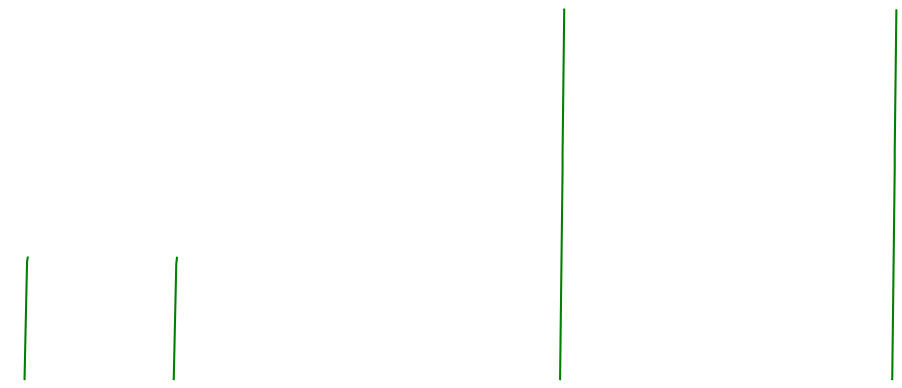

$$
\mid
$$

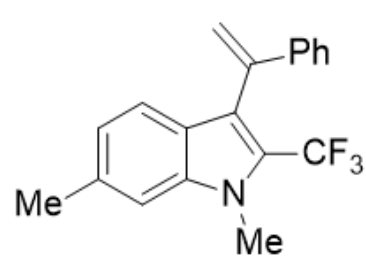

3ba; ${ }^{1} \mathrm{H}$ NMR

$\left(600 \mathrm{MHz}, \mathrm{CDCl}_{3}\right.$ )

1
네
808

м่ํ. 


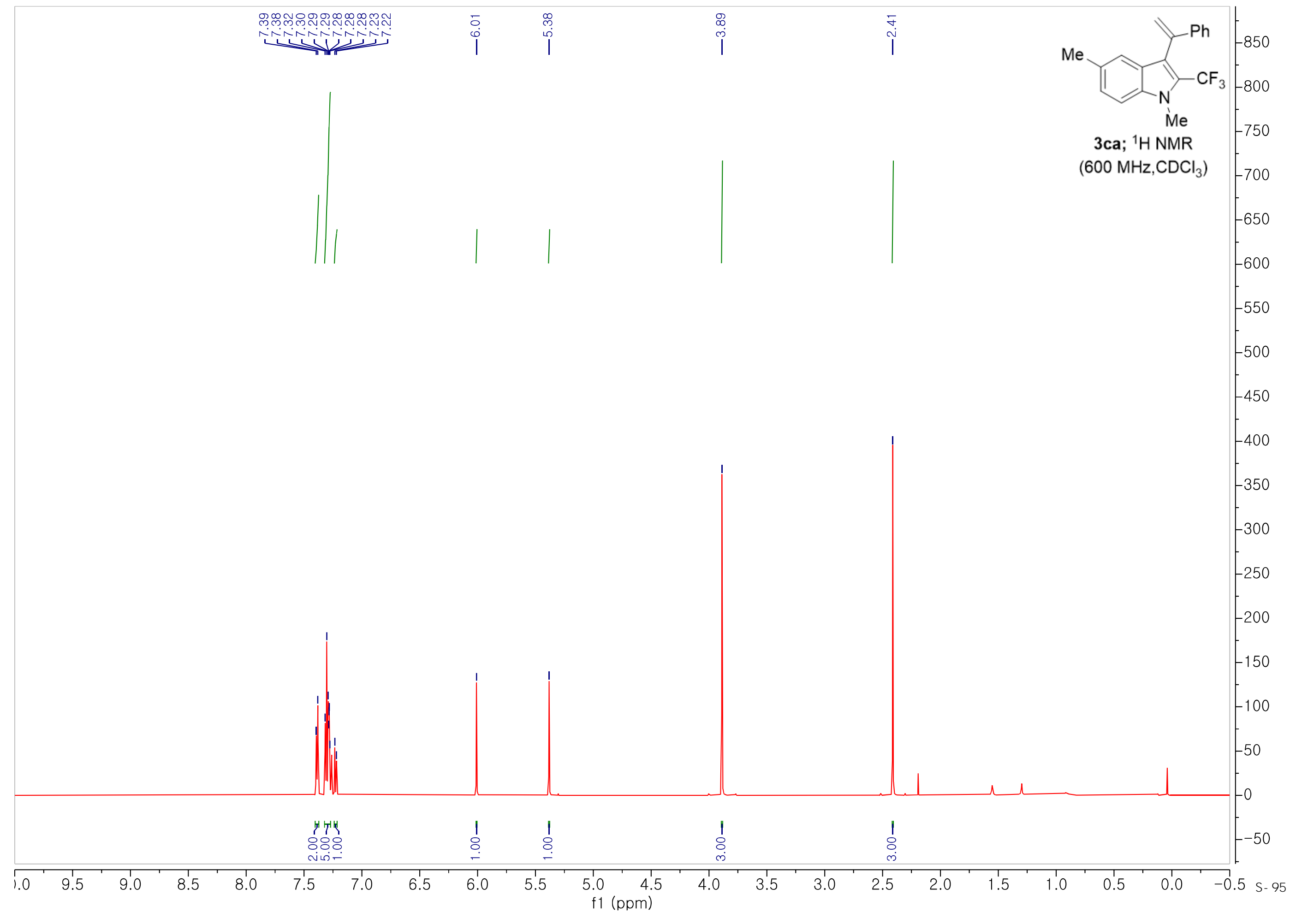




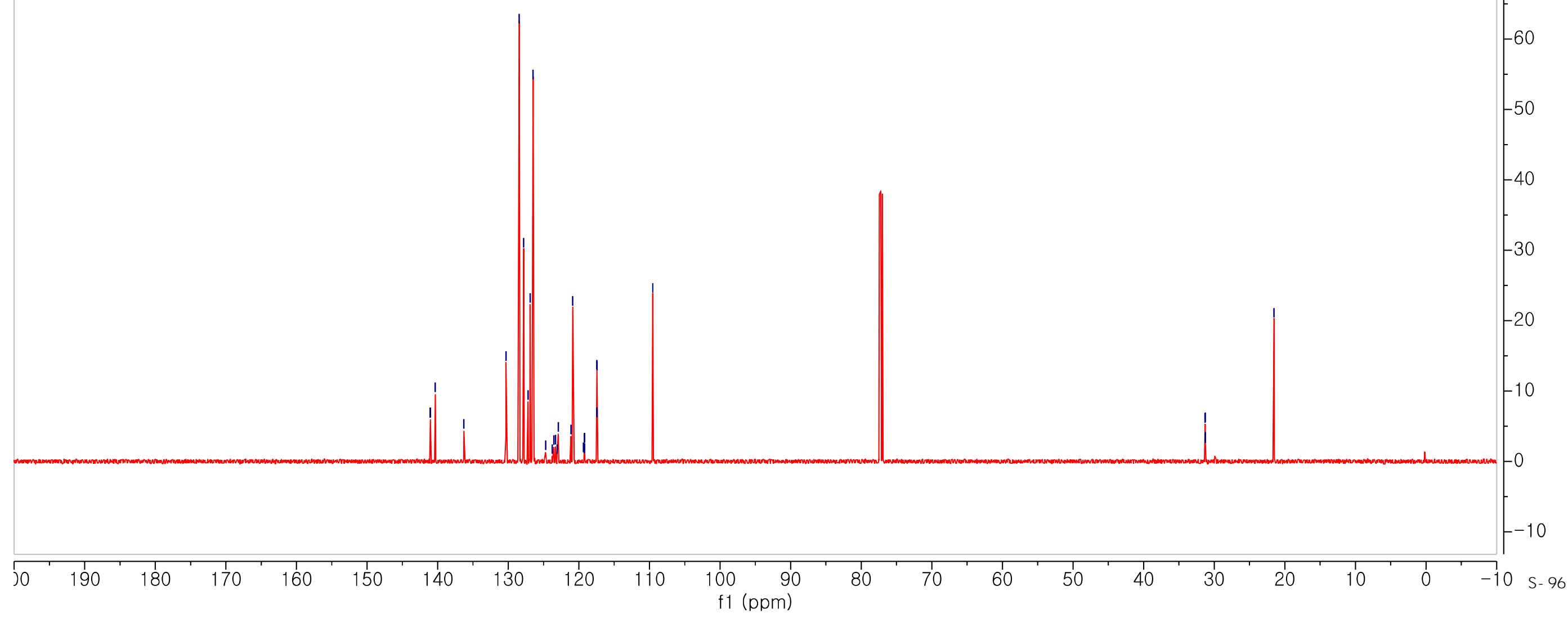



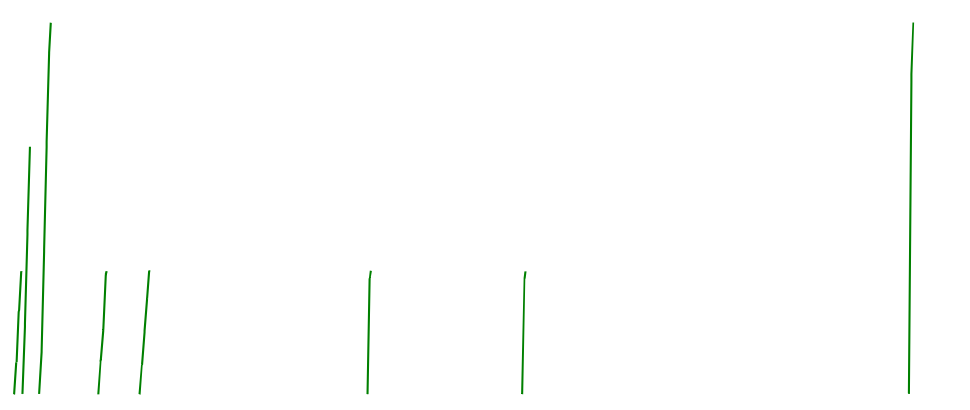

$\underbrace{\mathrm{Ph}}_{\mathrm{Me}} \mathrm{CF}_{-360}^{-420}$

3da; ${ }^{1} \mathrm{H}$ NMR

$-340$

$\left(600 \mathrm{MHz}, \mathrm{CDCl}_{3}\right)$

$-320$

$-300$

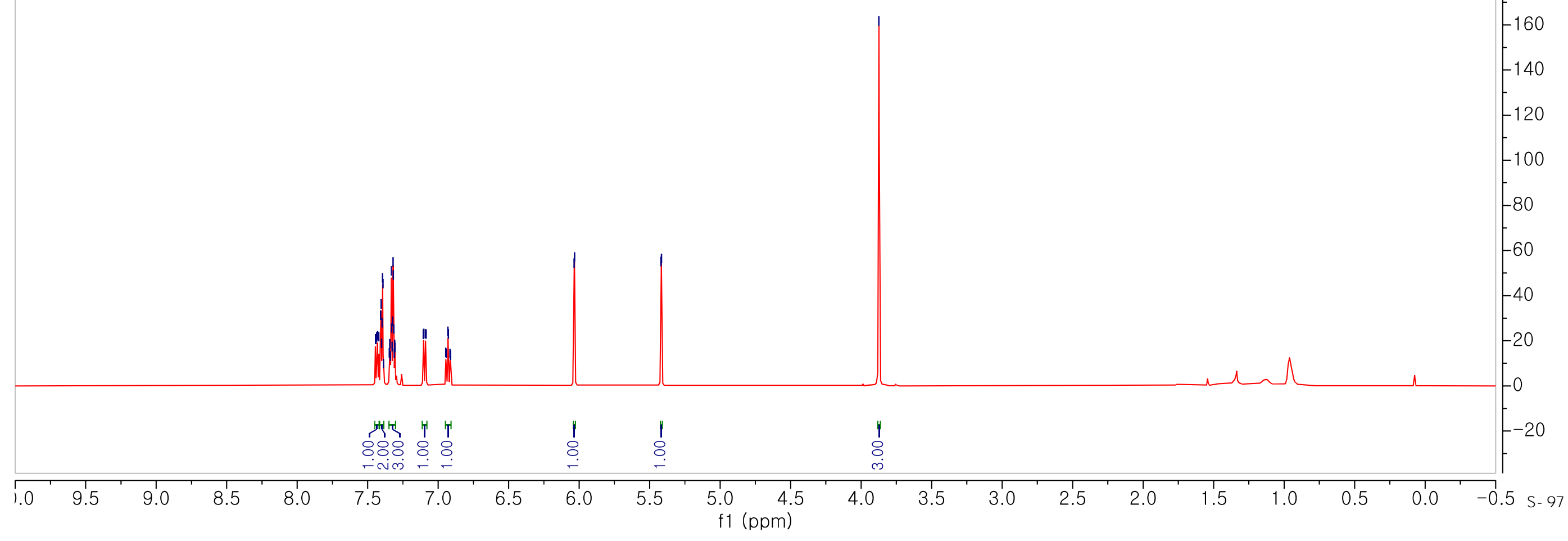




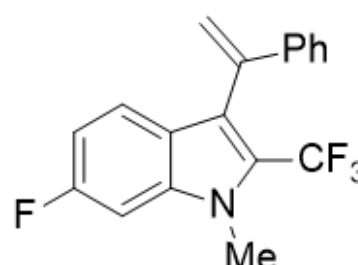

Me

3da; ${ }^{13} \mathrm{C}$ NMR $\left(151 \mathrm{MHz}, \mathrm{CDCl}_{3}\right.$ )

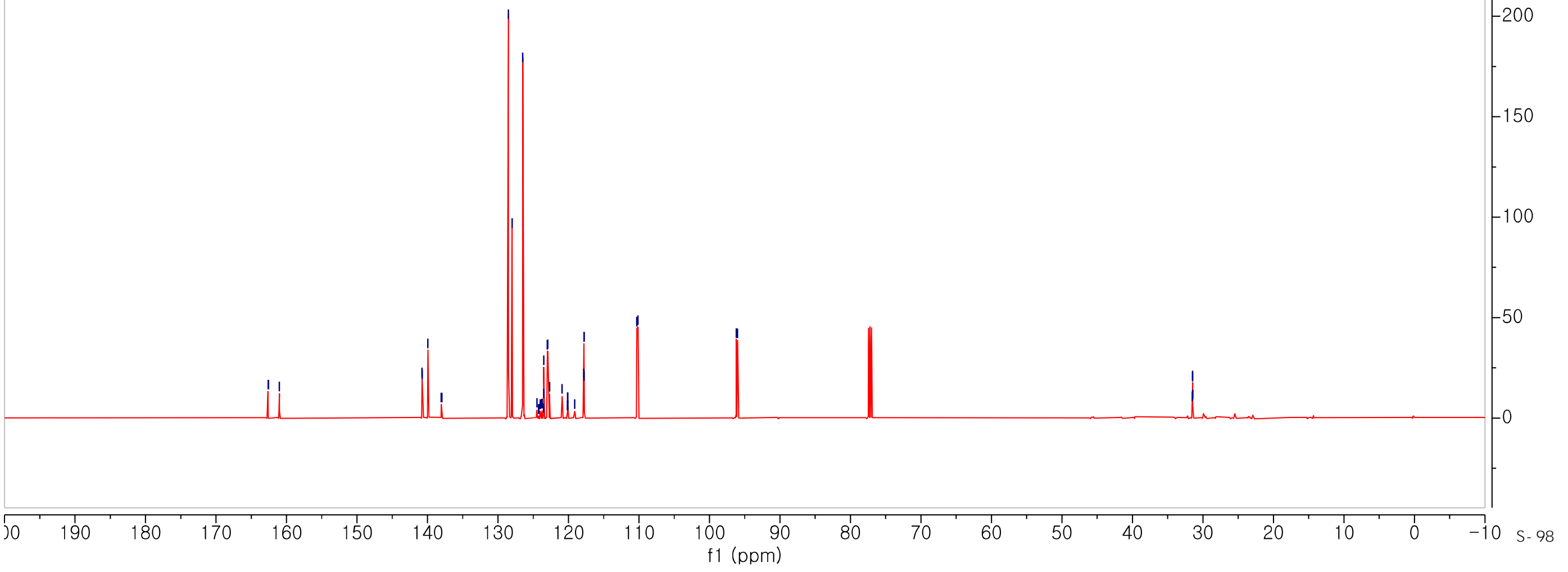



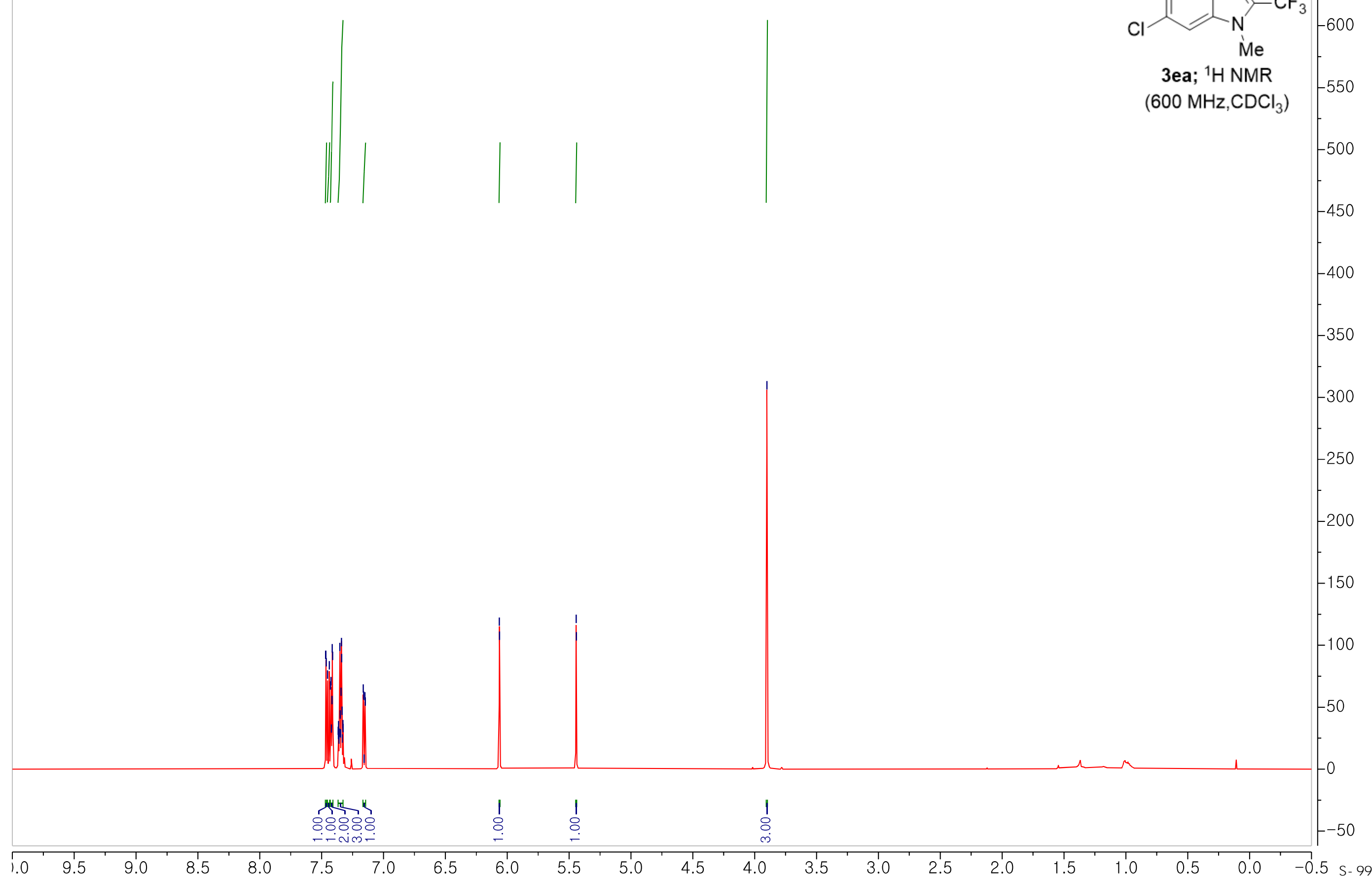

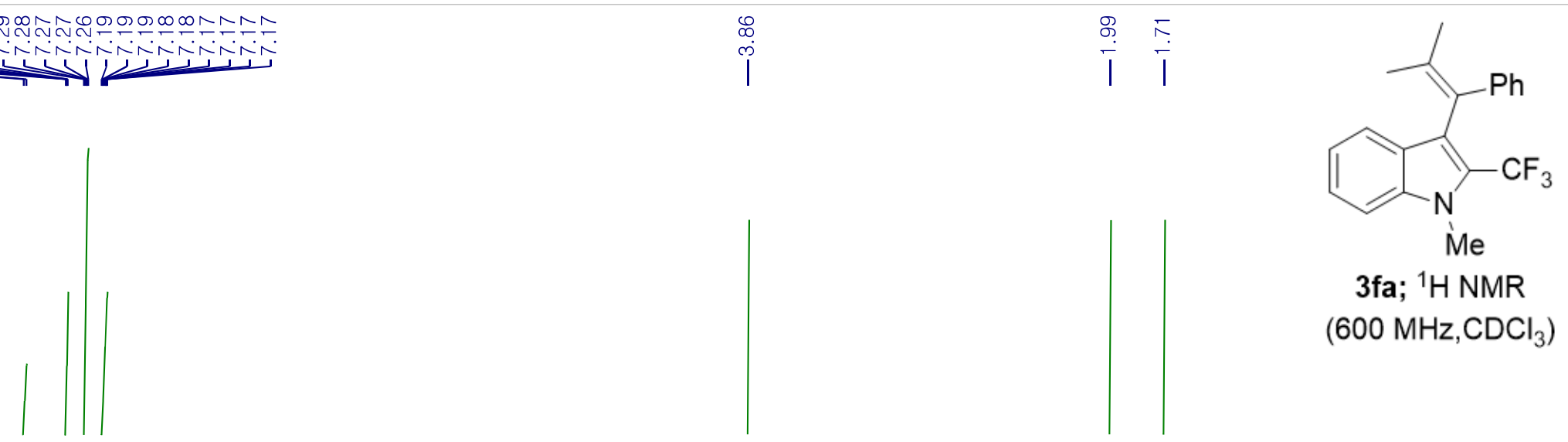

3fa; ${ }^{1} \mathrm{H}$ NMR (600 MHz, $\mathrm{CDCl}_{3}$ )

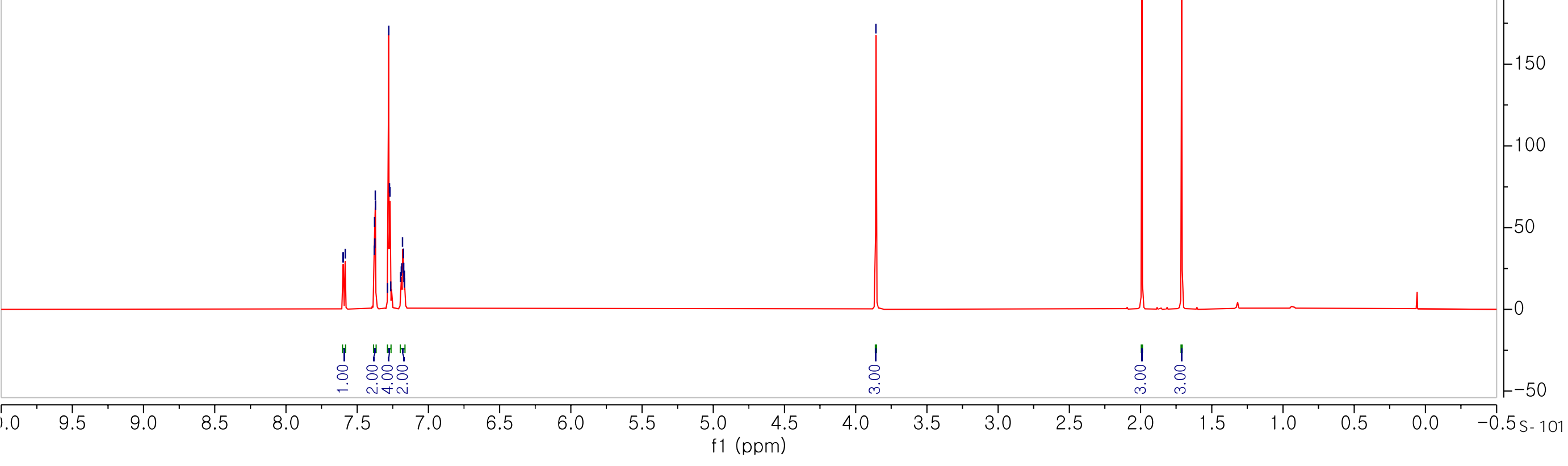




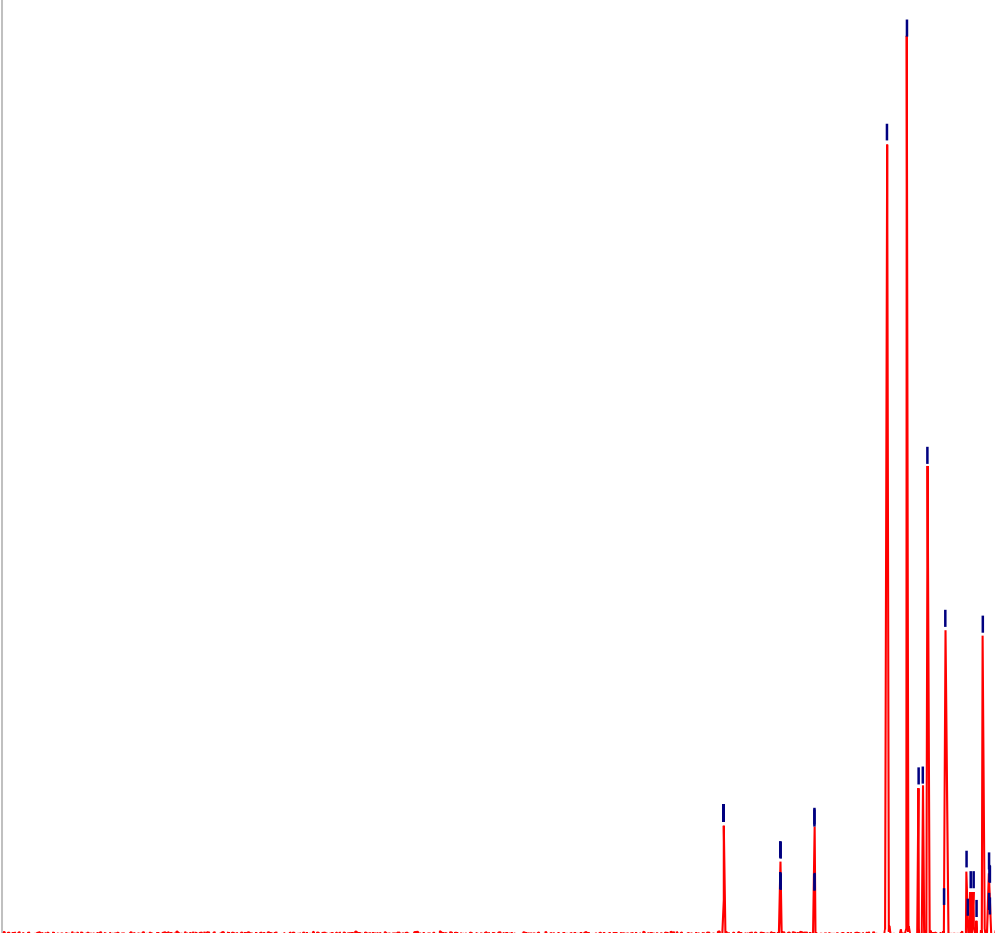

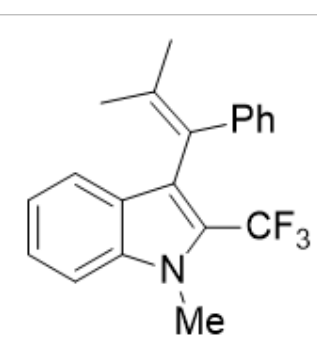

$3 \mathbf{f a} ;{ }^{13} \mathrm{C}$ NMR $\left(151 \mathrm{MHz}, \mathrm{CDCl}_{3}\right.$ ) 

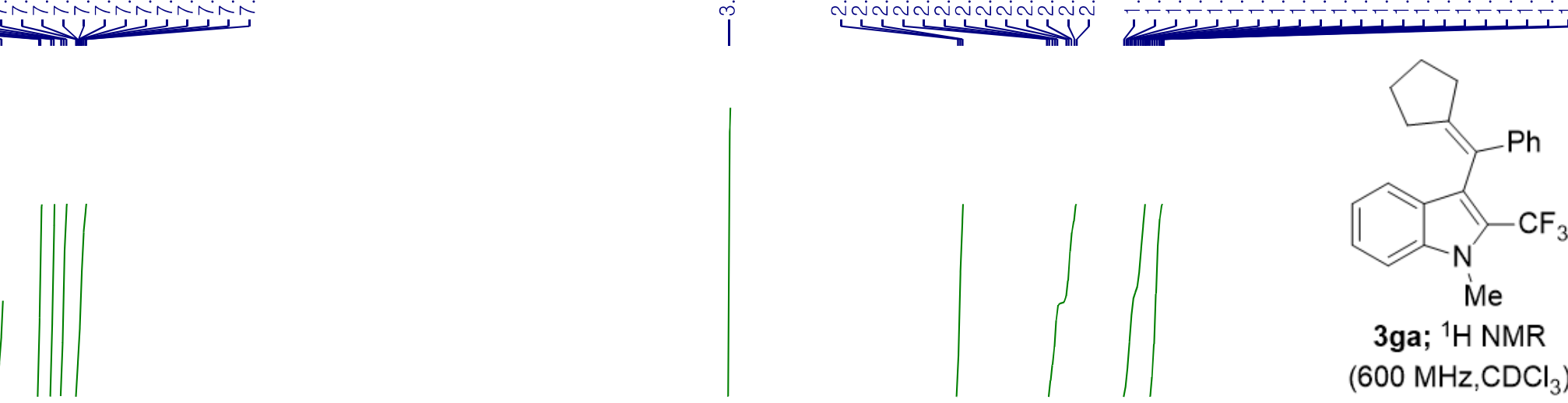


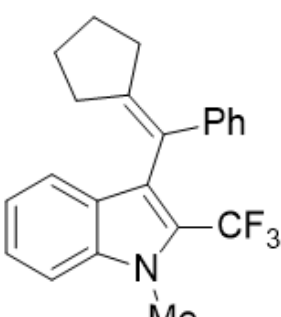

Me

3ga; ${ }^{13} \mathrm{C}$ NMR $\left(151 \mathrm{MHz}, \mathrm{CDCl}_{3}\right)$ 

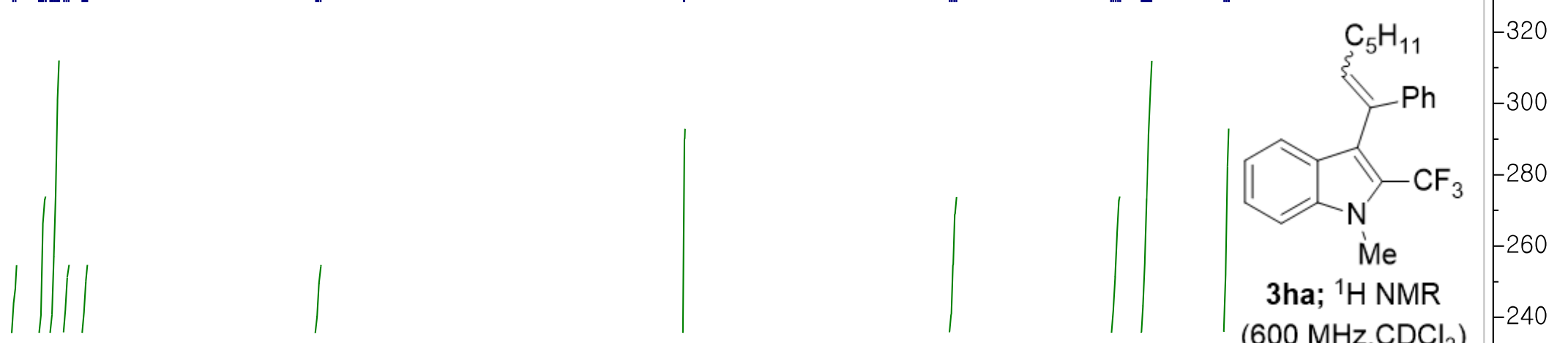

3ha; ${ }^{1} \mathrm{H}$ NMR $\left(600 \mathrm{MHz}, \mathrm{CDCl}_{3}\right)$

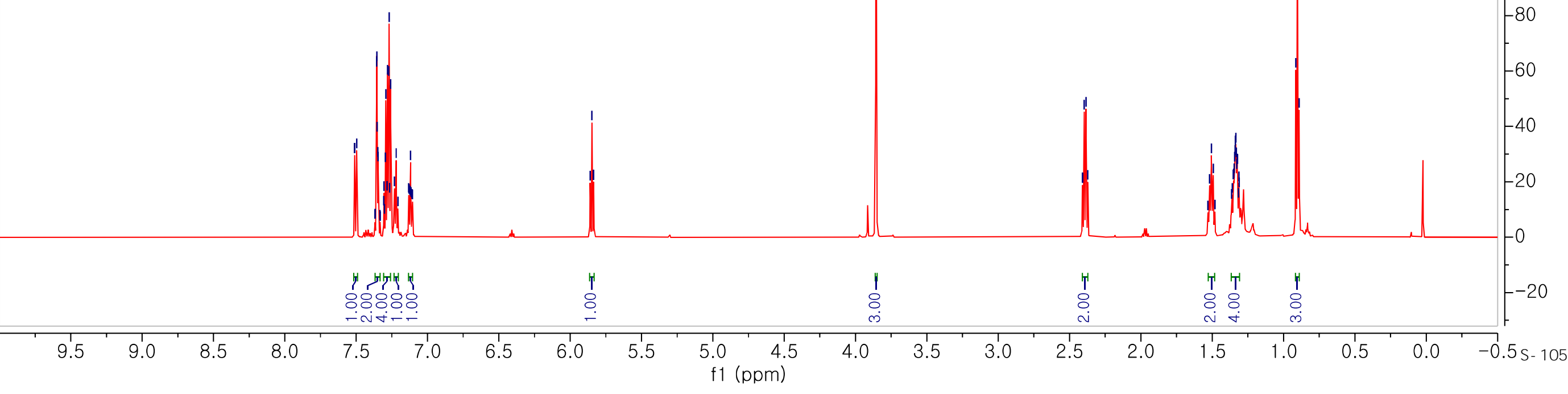



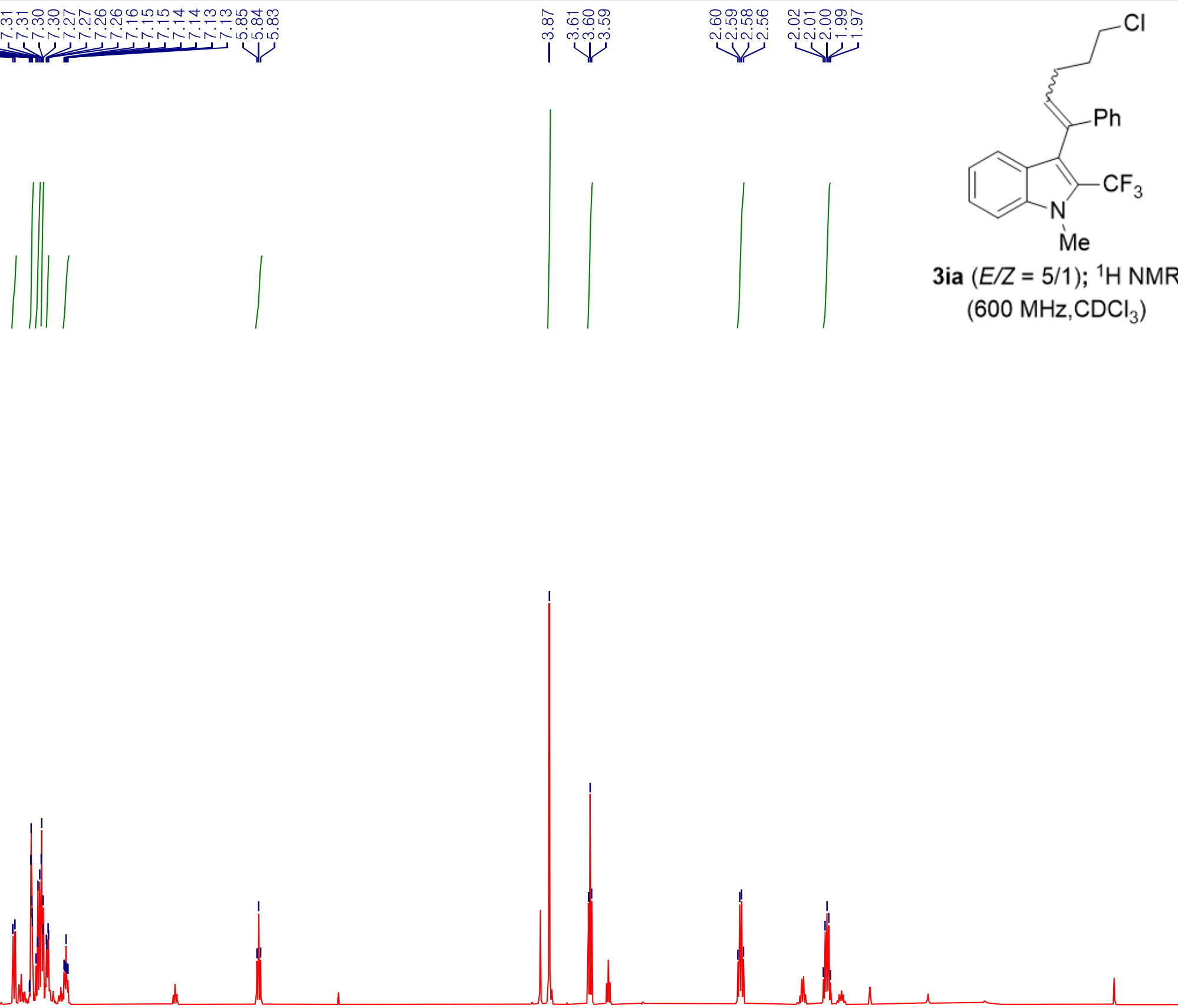

은응
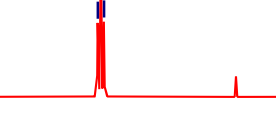

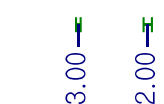

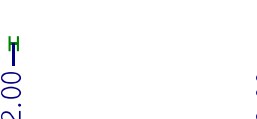




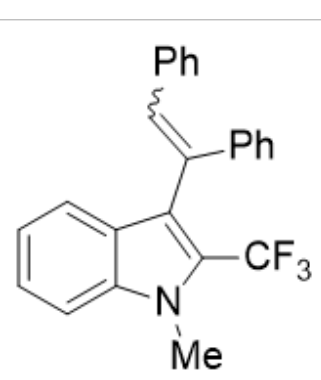

$3 \mathbf{j a} ;{ }^{13} \mathrm{C}$ NMR $\left(151 \mathrm{MHz}, \mathrm{CDCl}_{3}\right.$ )

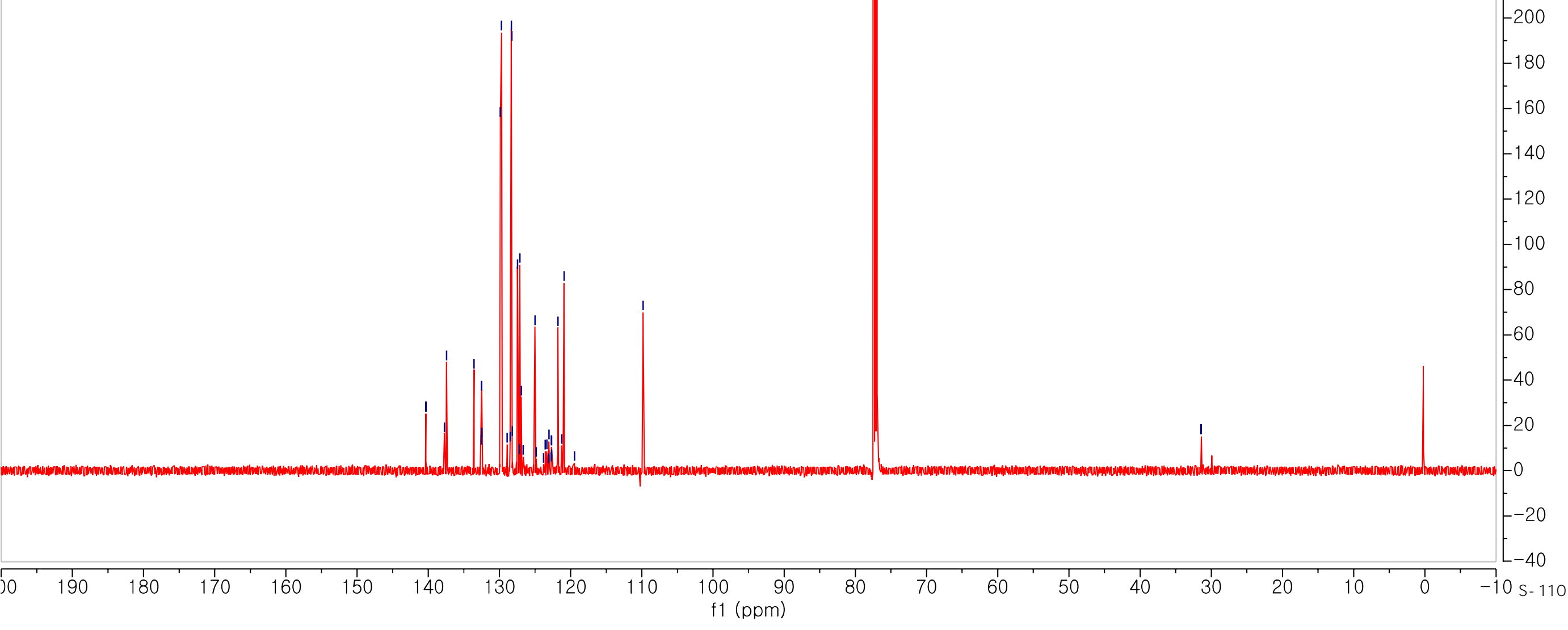




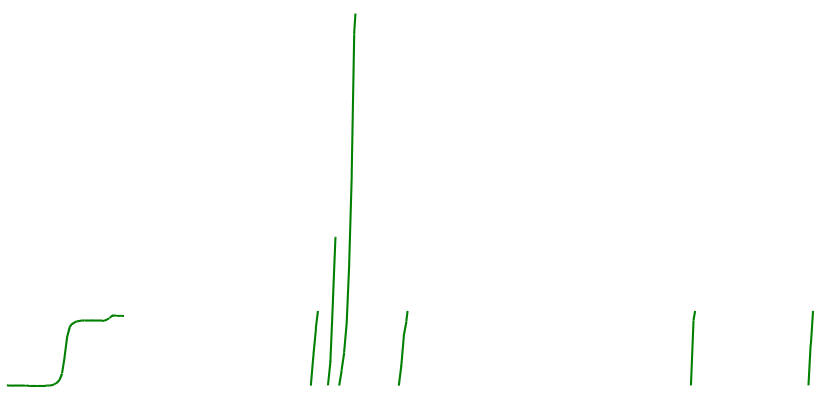



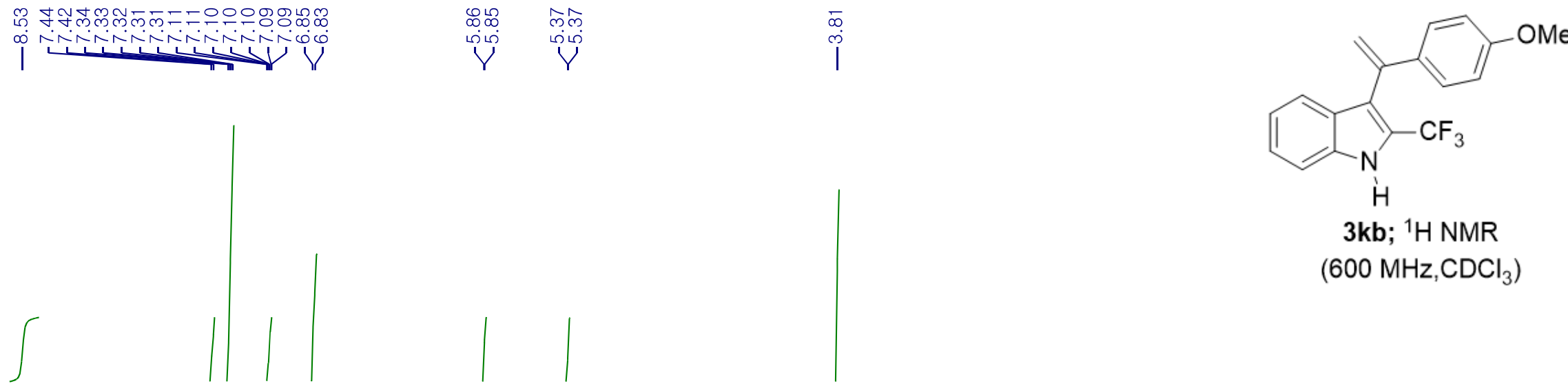

$\mathrm{H}$

3kb; ${ }^{1} \mathrm{H}$ NMR

$\left(600 \mathrm{MHz}, \mathrm{CDCl}_{3}\right)$

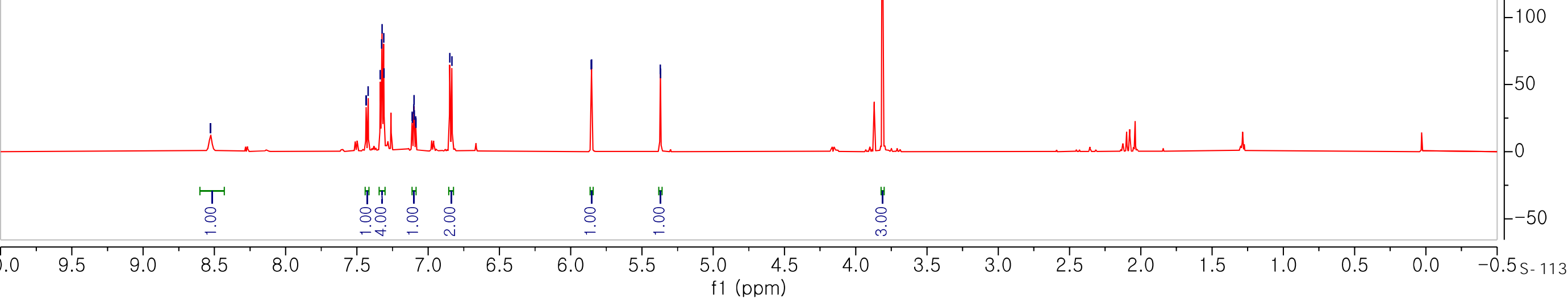




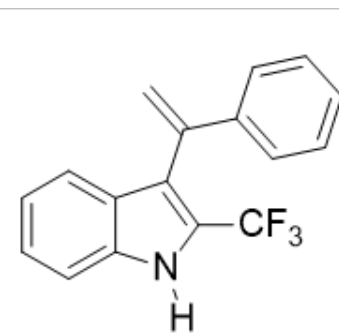

$3 \mathbf{k b} ;{ }^{13} \mathrm{C}$ NMR $\left(151 \mathrm{MHz}, \mathrm{CDCl}_{3}\right)$

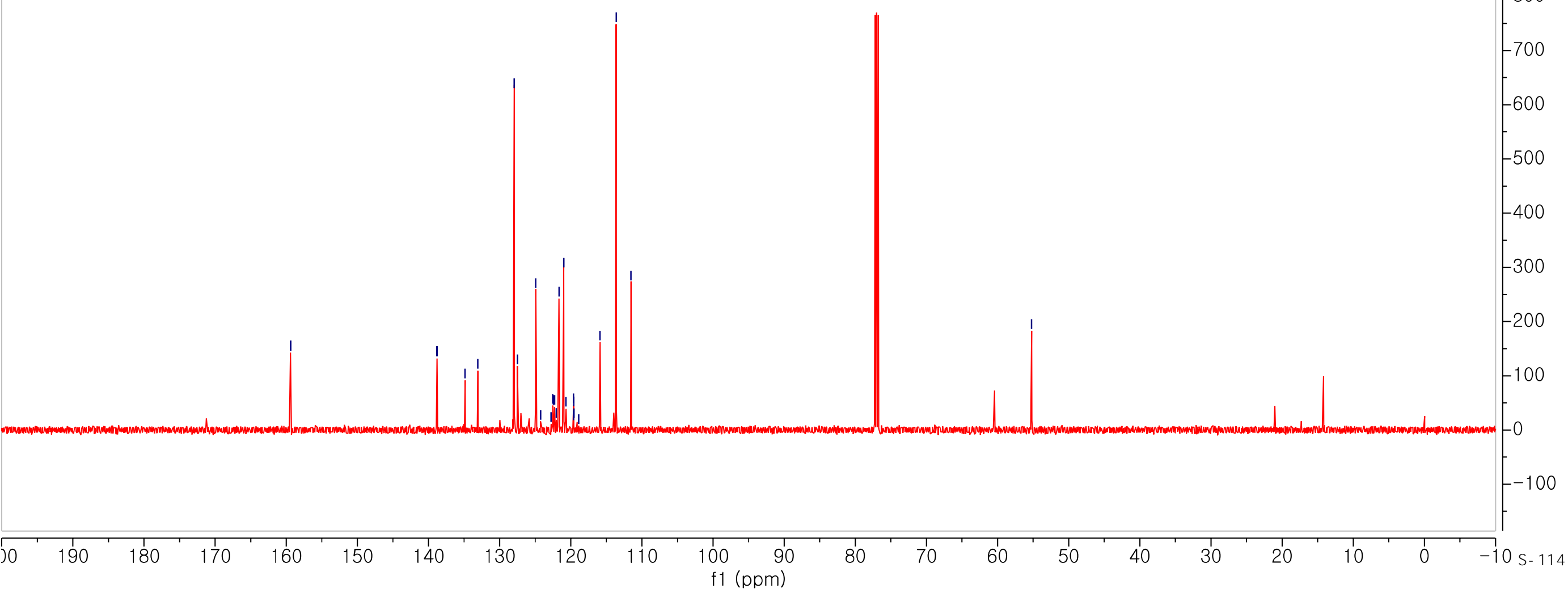




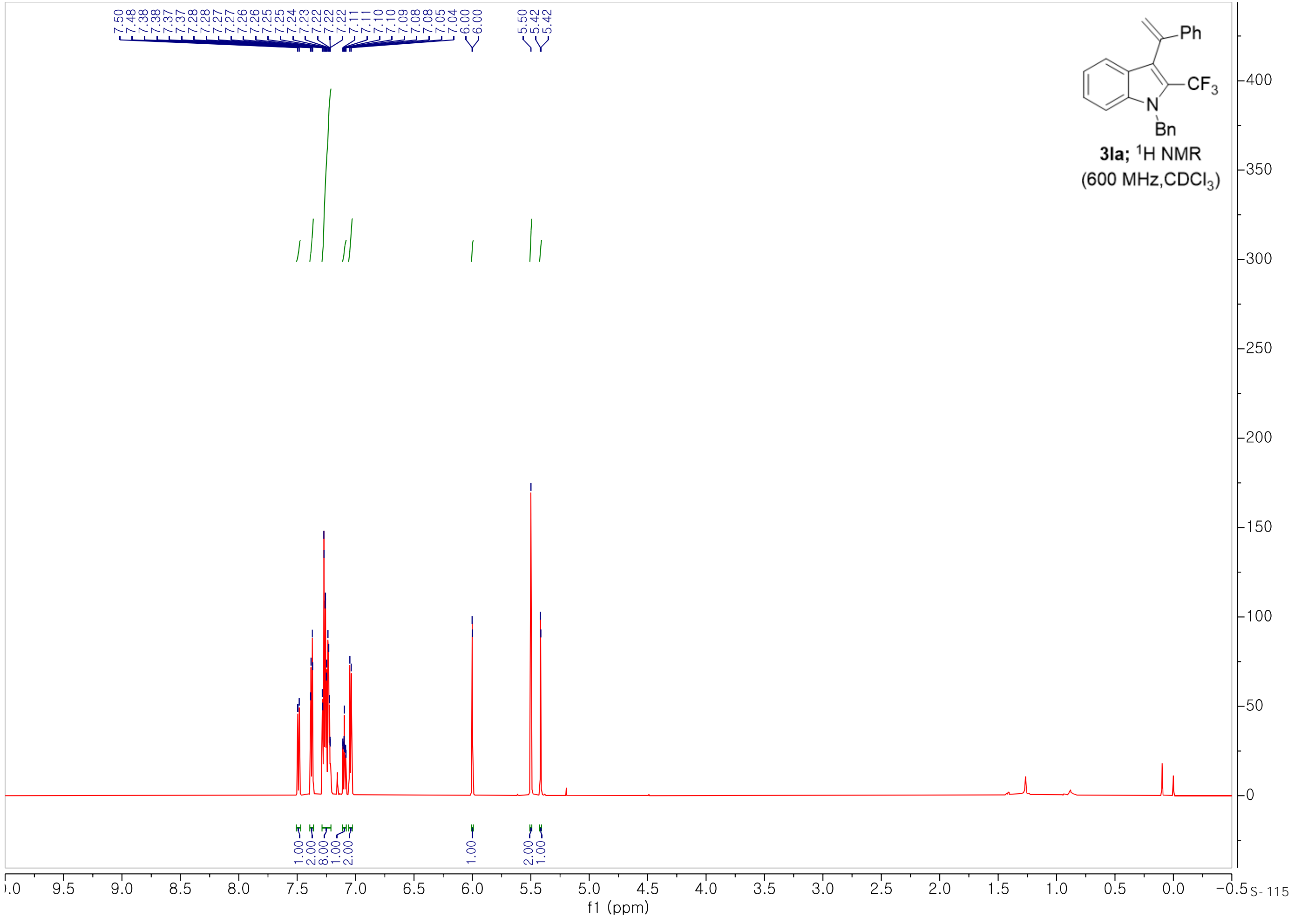


स 

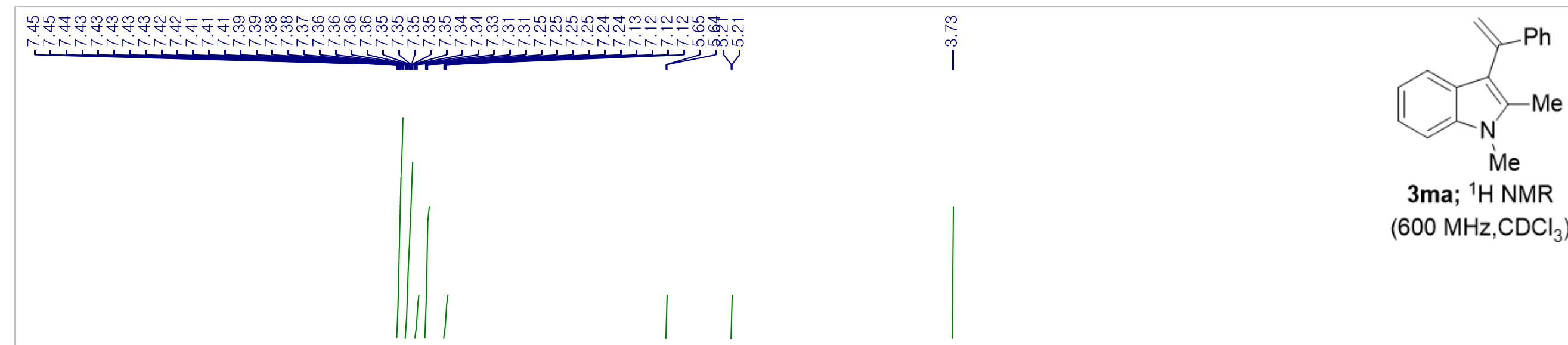

3ma; ${ }^{1} \mathrm{H}$ NMR $\left(600 \mathrm{MHz}, \mathrm{CDCl}_{3}\right)$ $\left.\mathrm{DCl}_{3}\right)$ 


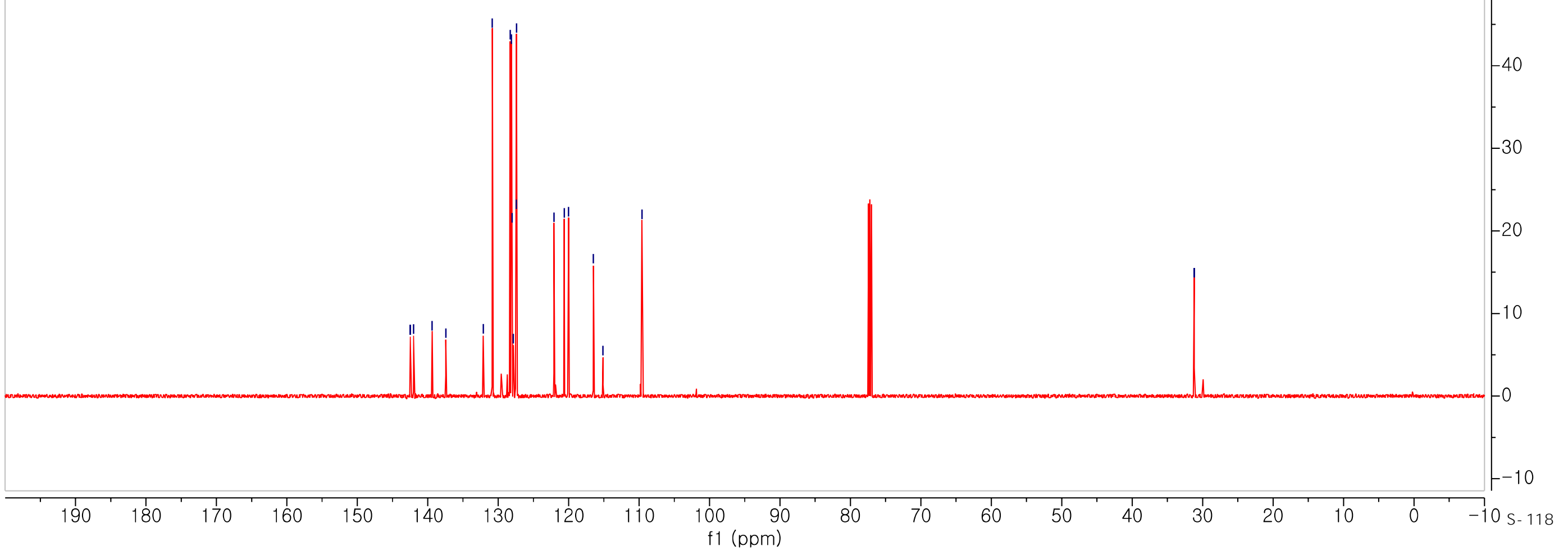



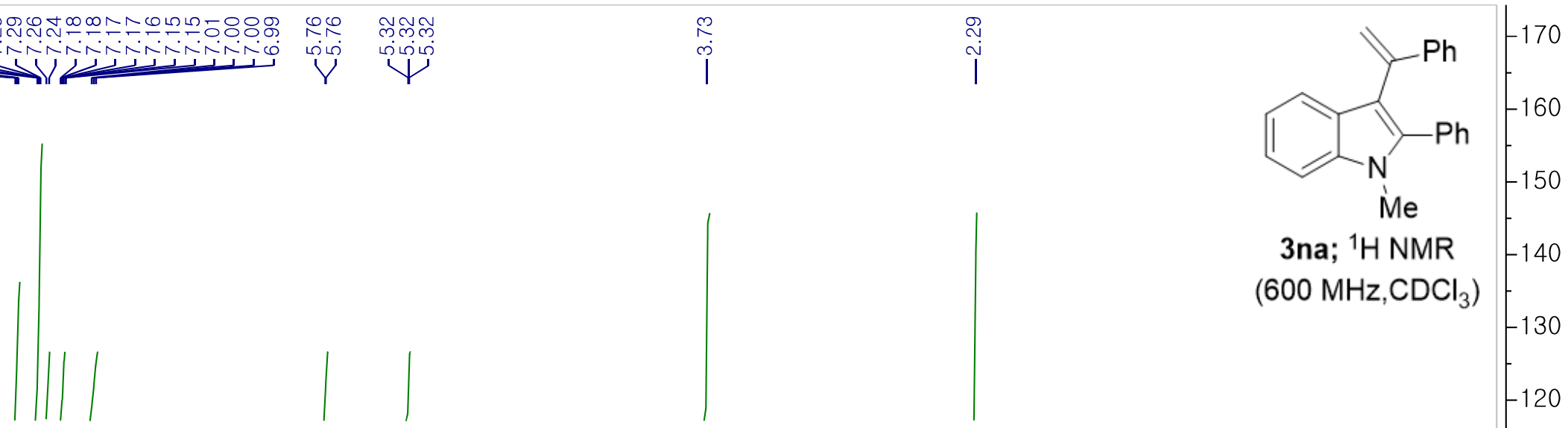

3na; ${ }^{1} \mathrm{H}$ NMR -140 $\left(600 \mathrm{MHz}, \mathrm{CDCl}_{3}\right.$ )

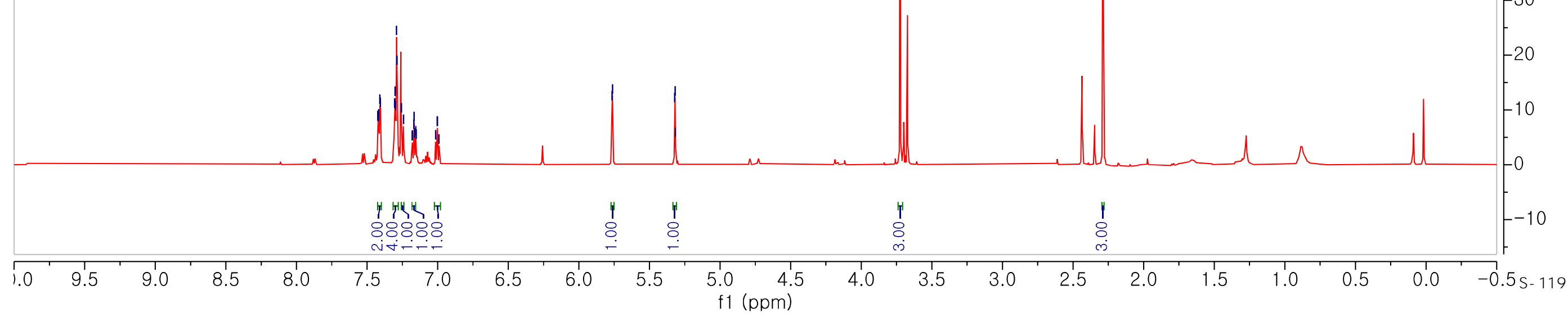




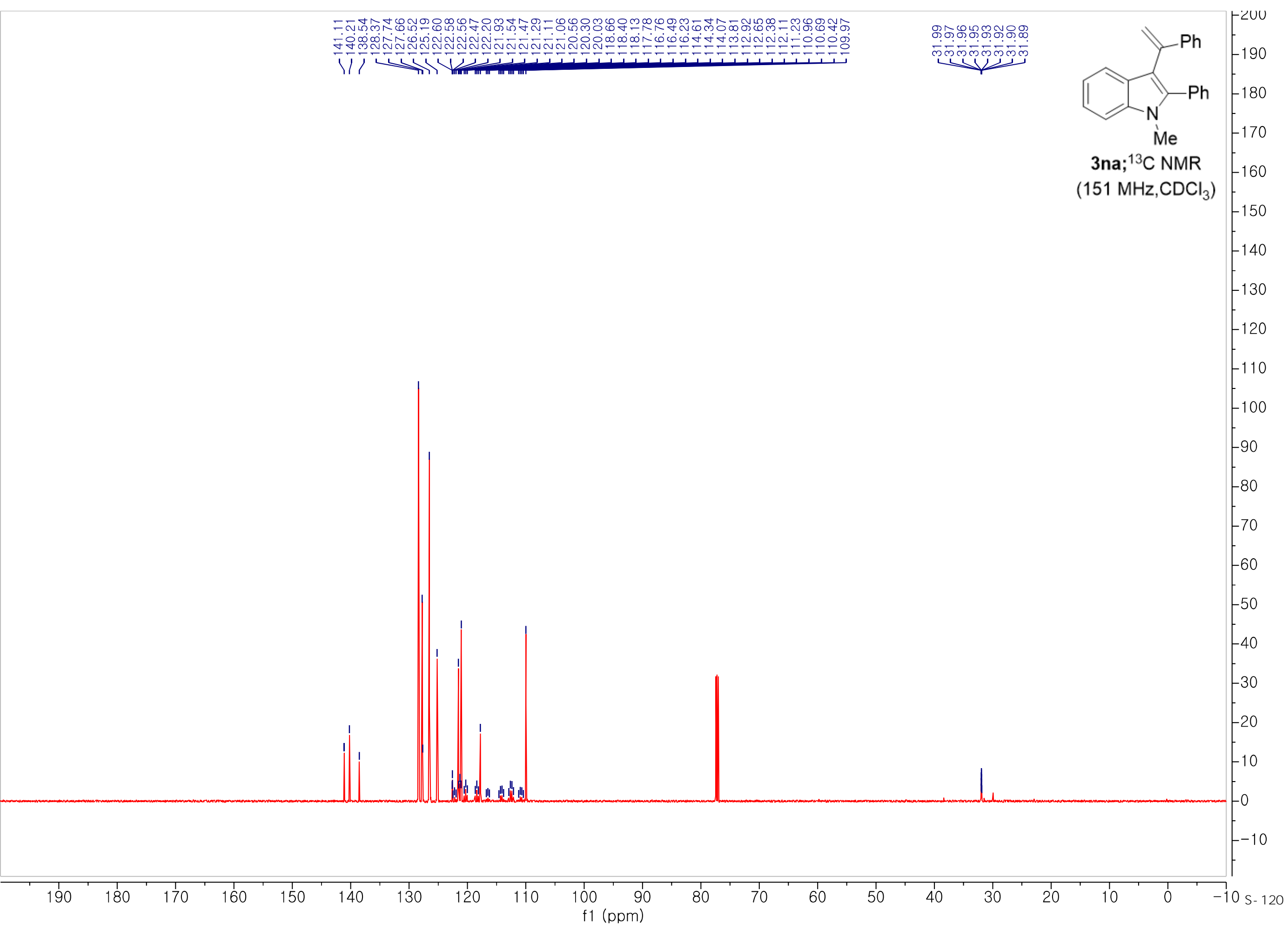



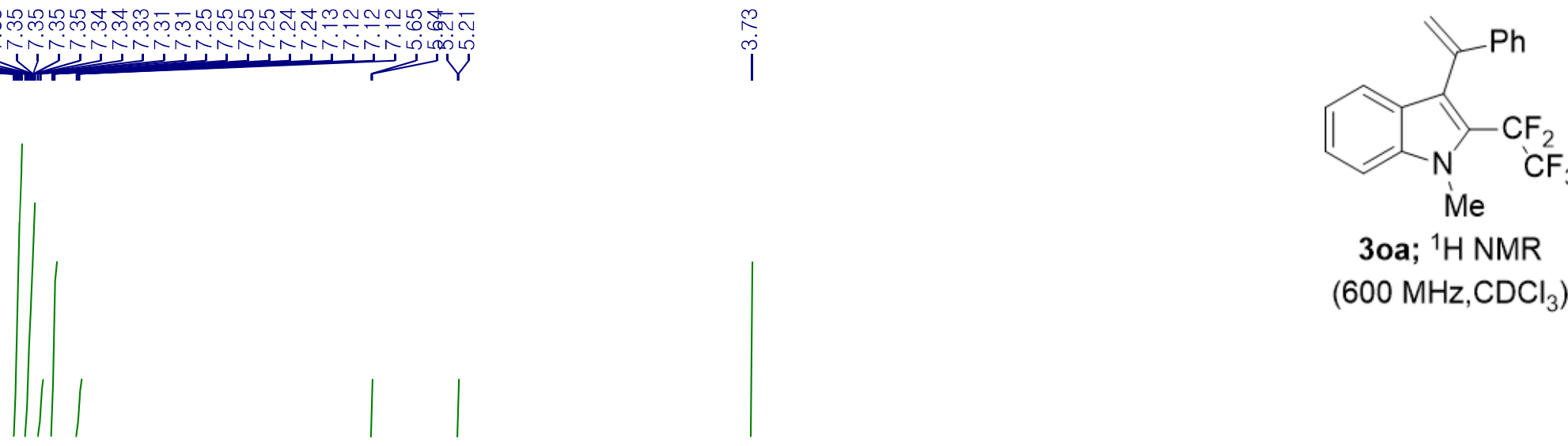

3oa: ${ }^{1} \mathrm{H}$ NMR $-350$ $\left(600 \mathrm{MHz}, \mathrm{CDCl}_{3}\right.$ )

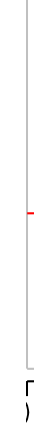

.

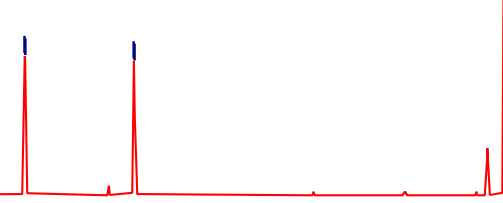


3oa; ${ }^{13} \mathrm{C}$ NMR $\left(151 \mathrm{MHz}, \mathrm{CDCl}_{3}\right.$ ) 


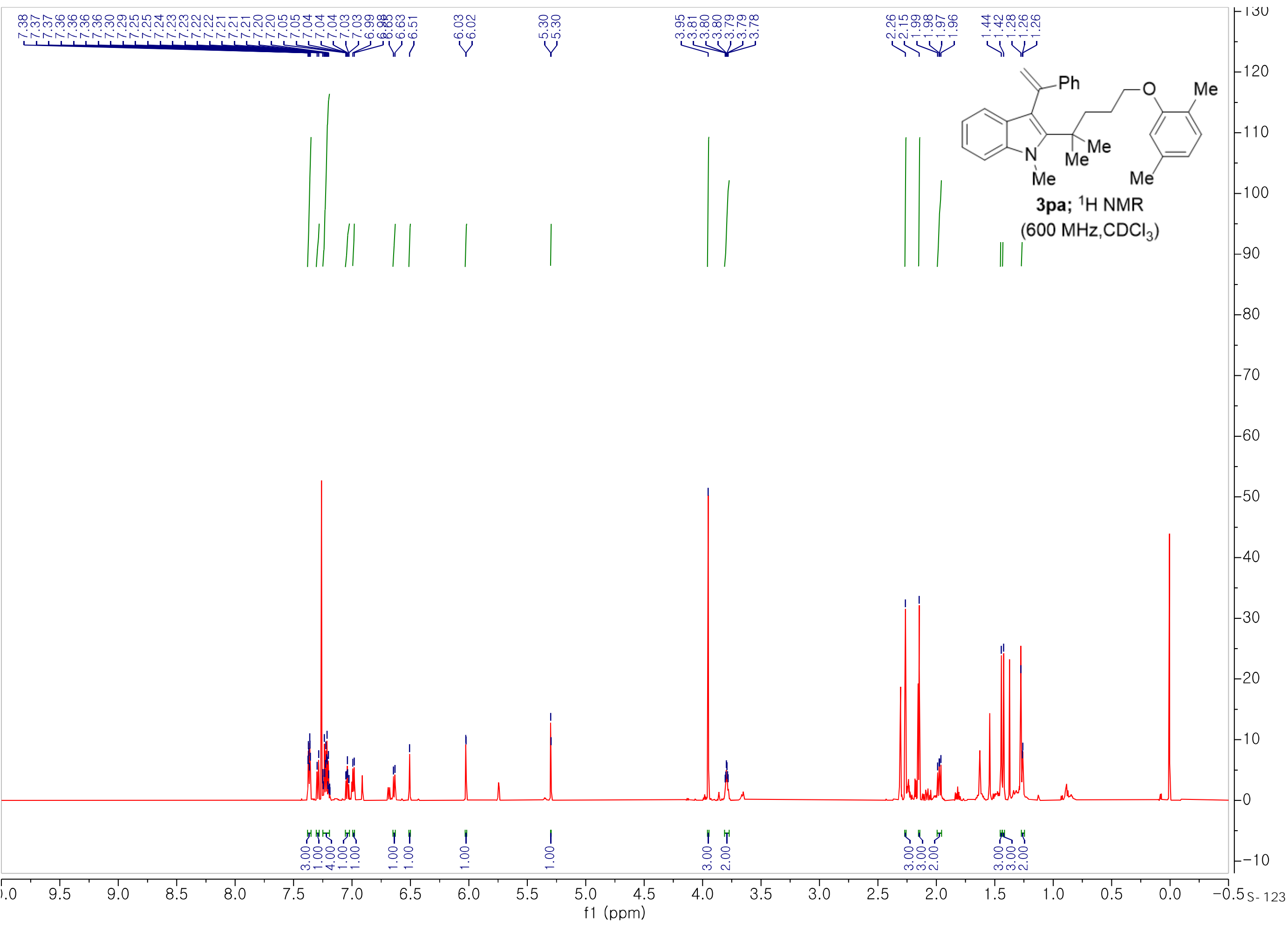




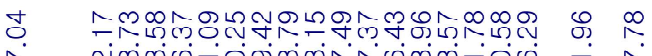



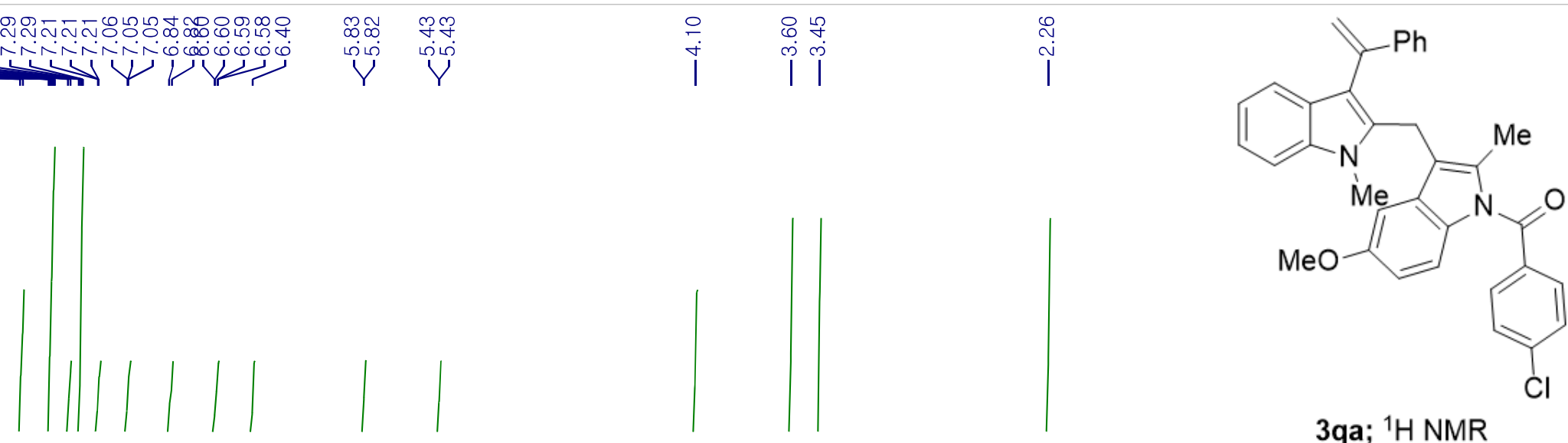

3qa; ${ }^{1} \mathrm{H}$ NMR

$-280$ $\left(600 \mathrm{MHz} \mathrm{CDCl}_{3}\right)$

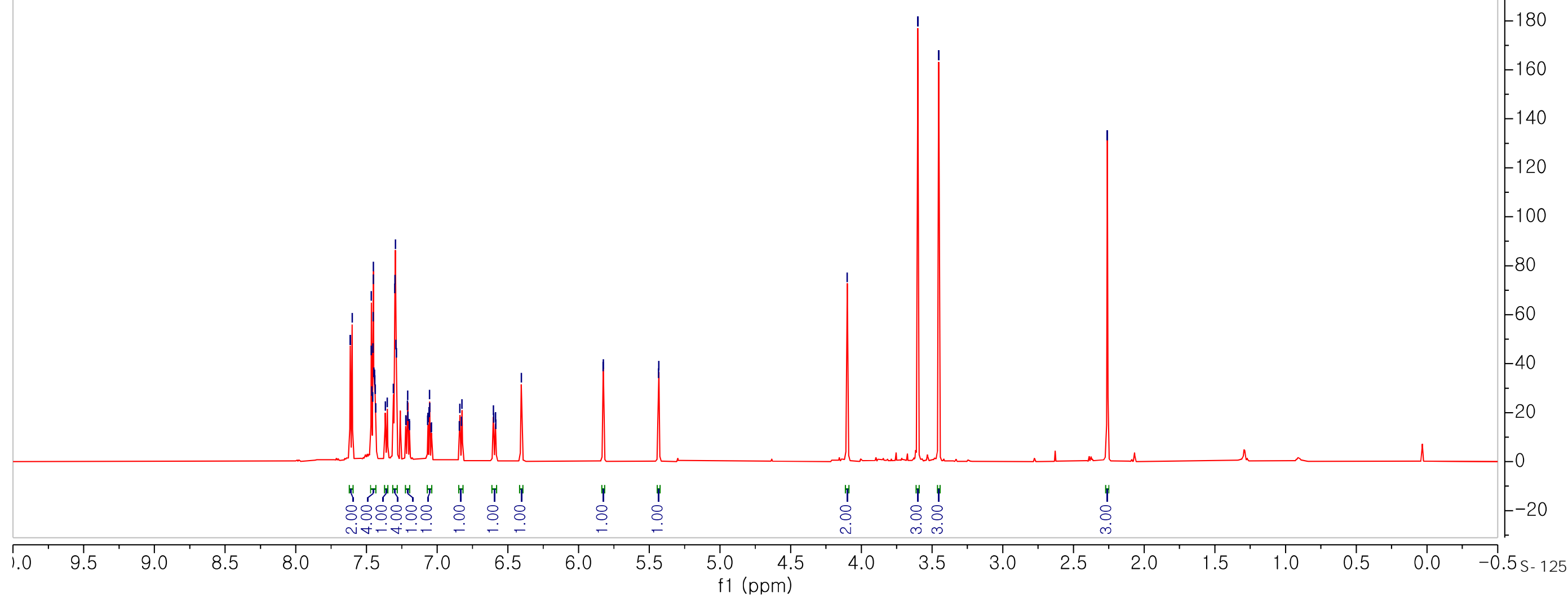



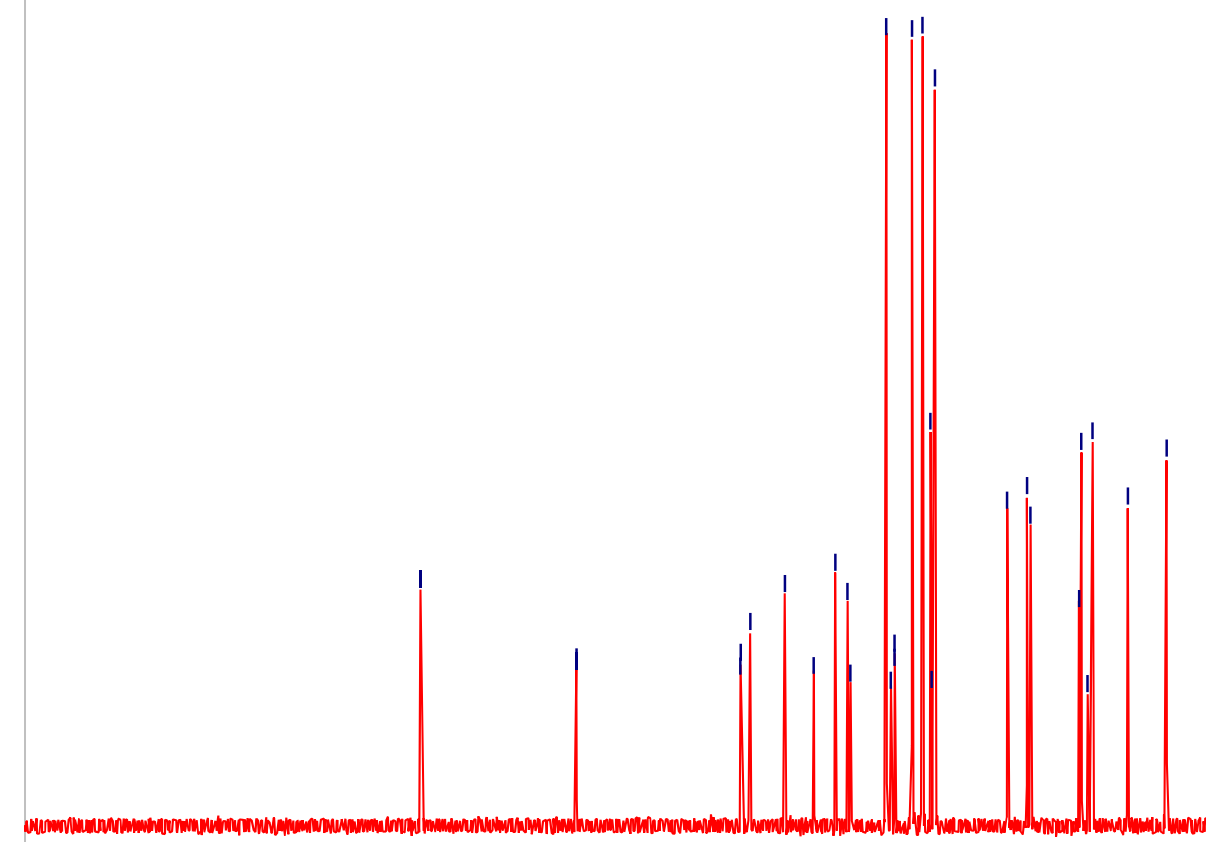

3qa; ${ }^{13} \mathrm{C}$ NMR $\left(151 \mathrm{MHz}, \mathrm{CDCl}_{3}\right.$ )<smiles>C=C(c1ccccc1)c1c(Cc2c(C)n(CC)c3ccc(OC)cc23)n(C)c2ccccc12</smiles> 


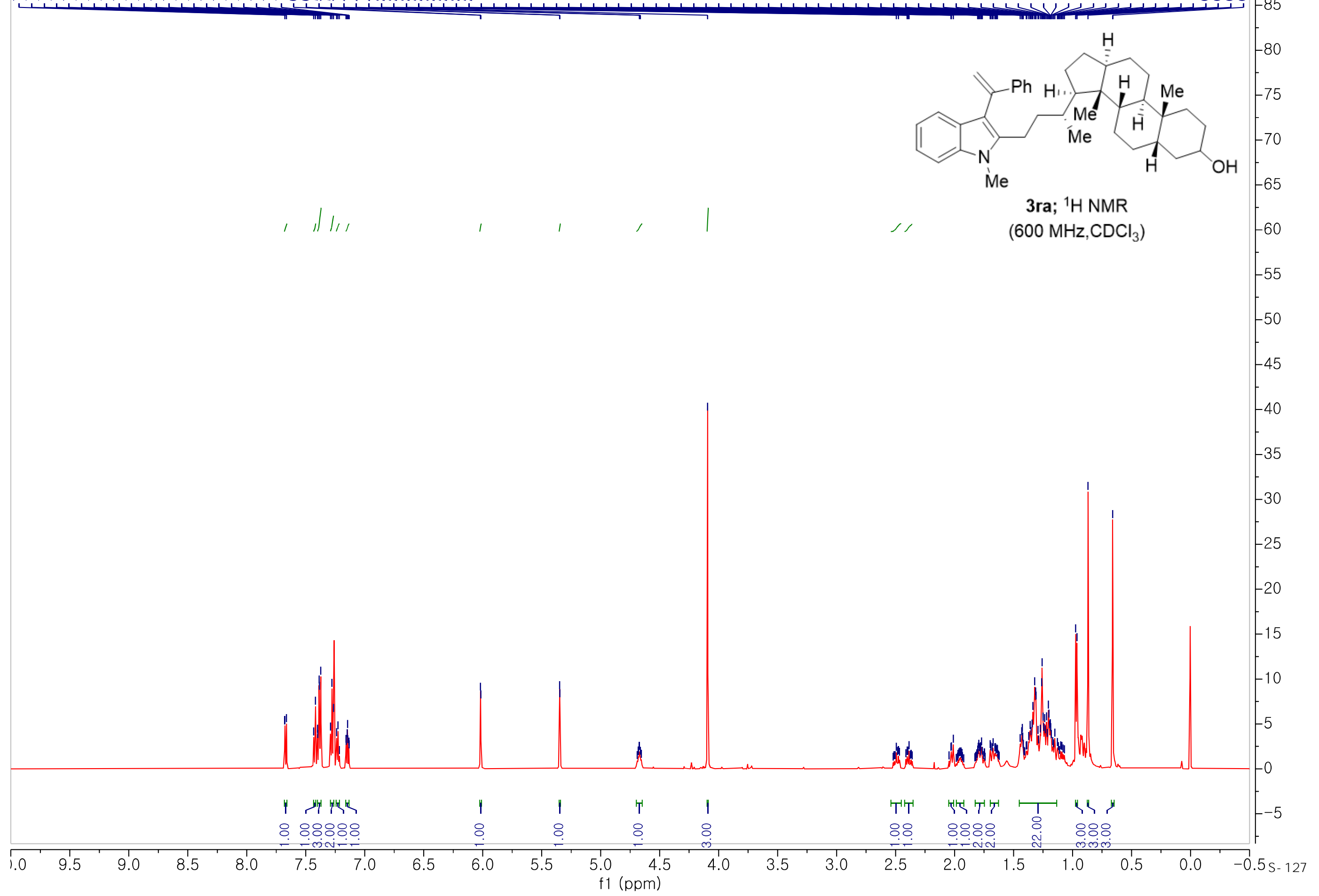




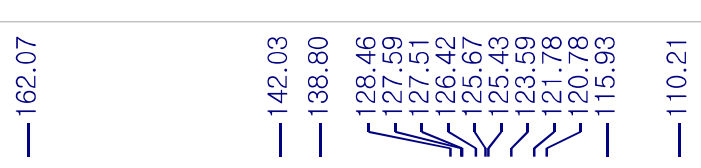
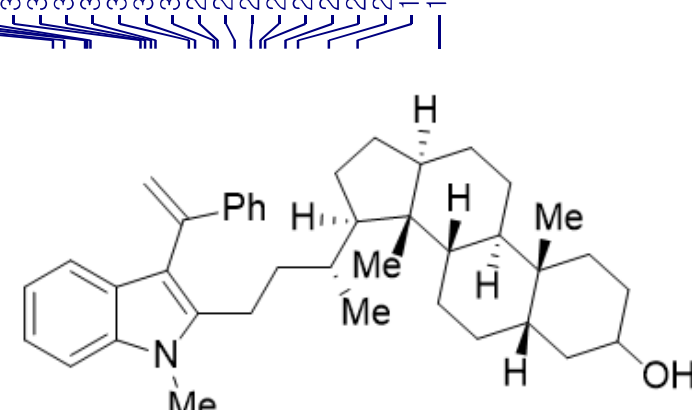

Me

$3 \mathrm{ra} ;{ }^{13} \mathrm{C}$ NMR (151 $\mathrm{MHz}, \mathrm{CDCl}_{3}$ )

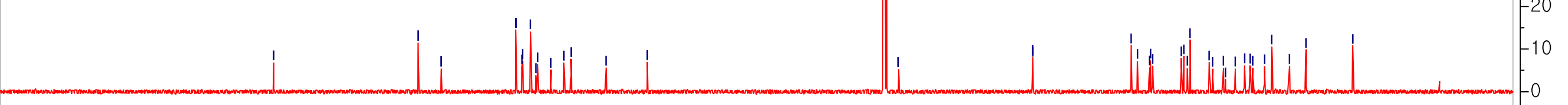




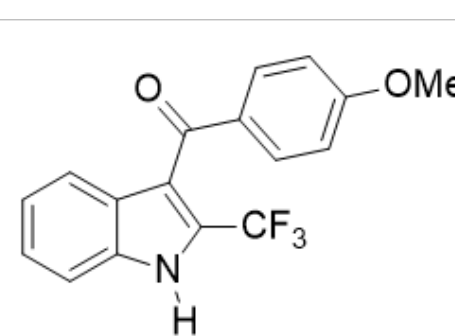

$\mathrm{H}$

5; ${ }^{1} \mathrm{H}$ NMR $\left(600 \mathrm{MHz}, \mathrm{CDCl}_{3}\right.$ )

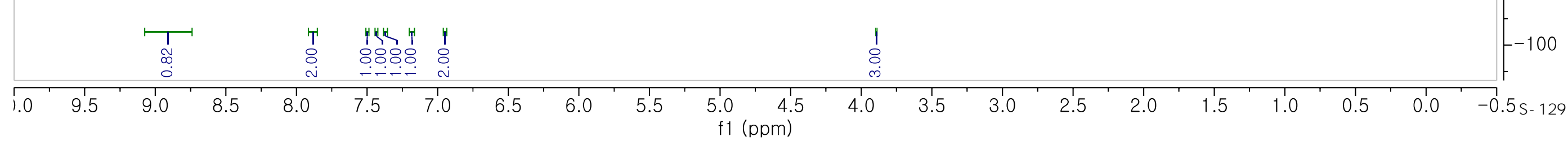




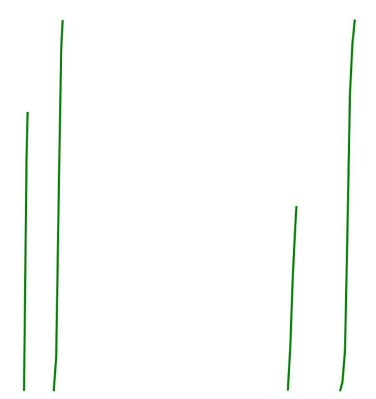

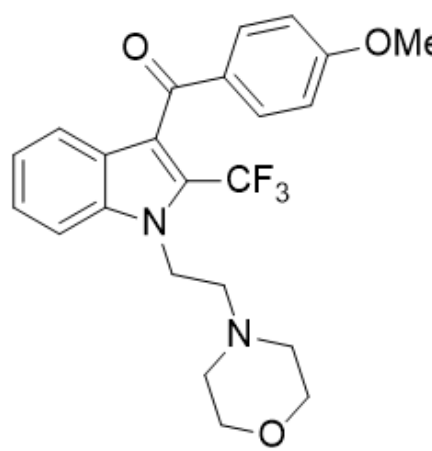

7; ${ }^{1} \mathrm{H}$ NMR $\left(600 \mathrm{MHz}, \mathrm{CDCl}_{3}\right.$ )

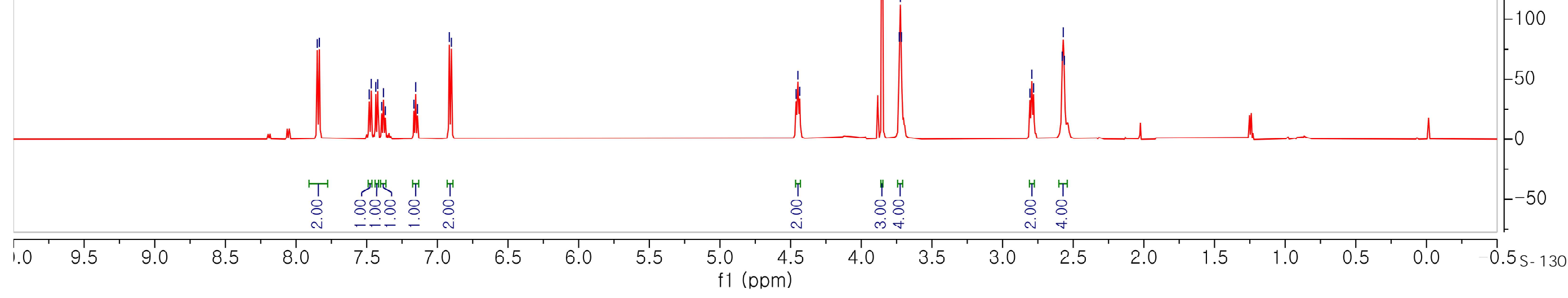


9a; ${ }^{1} \mathrm{H}$ NMR

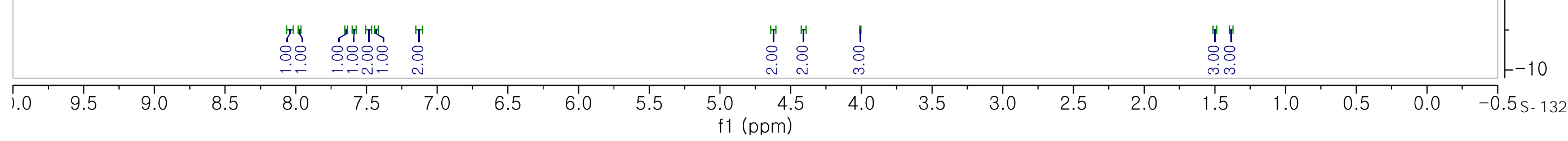




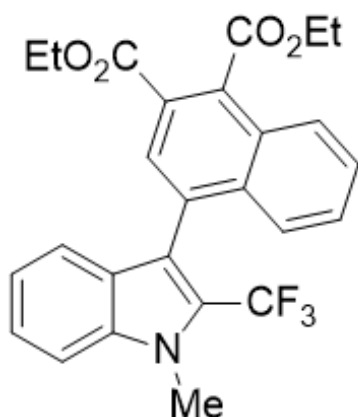

$9 a ;{ }^{13} \mathrm{C}$ NMR

$\left(151 \mathrm{MHz}, \mathrm{CDCl}_{3}\right)$

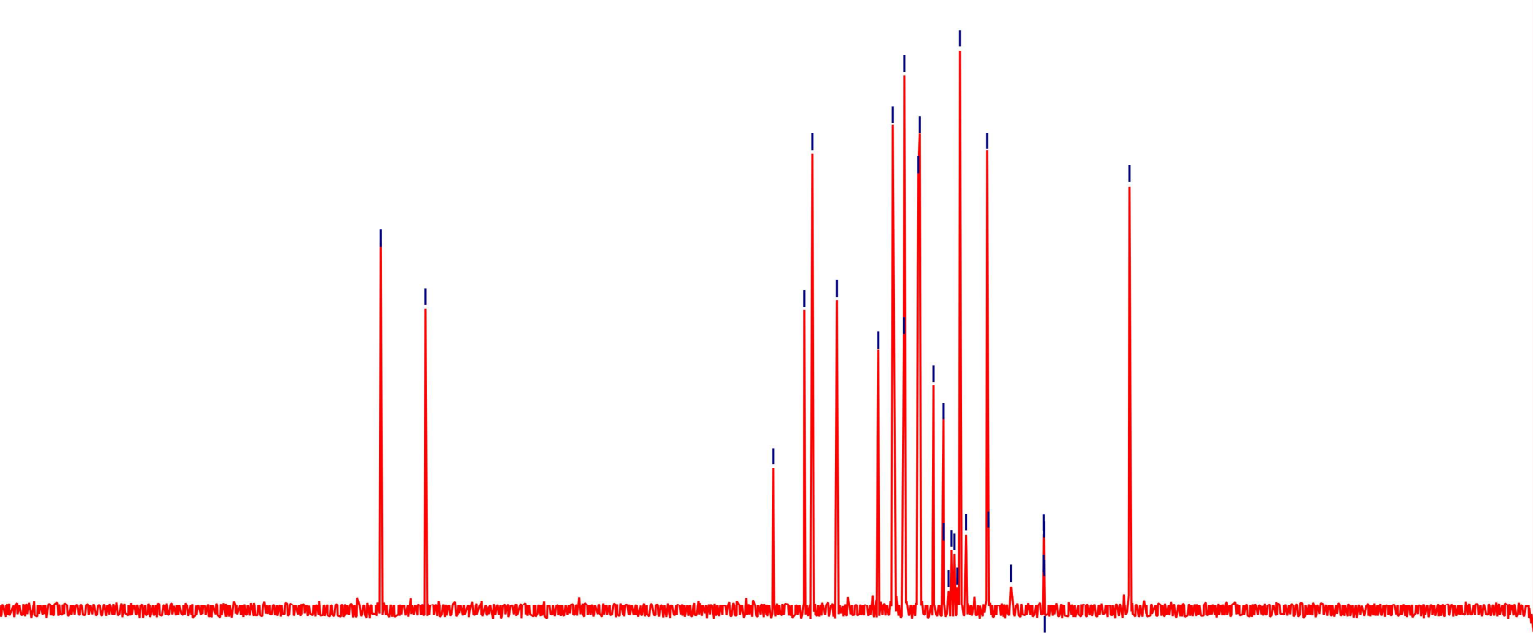

$190 \quad 180$

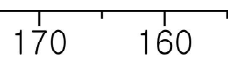

150

$140 \quad 130$

120

110 

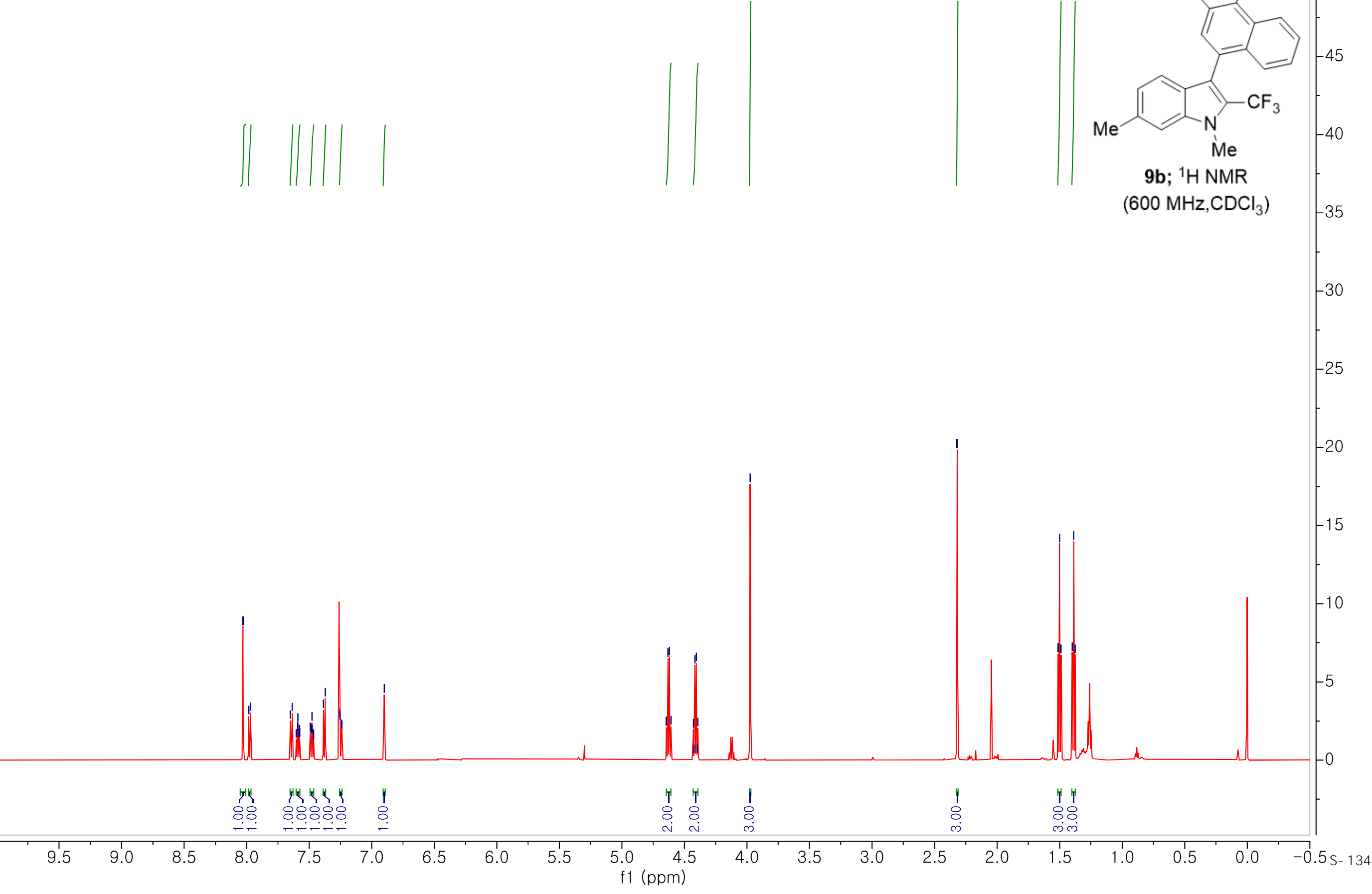

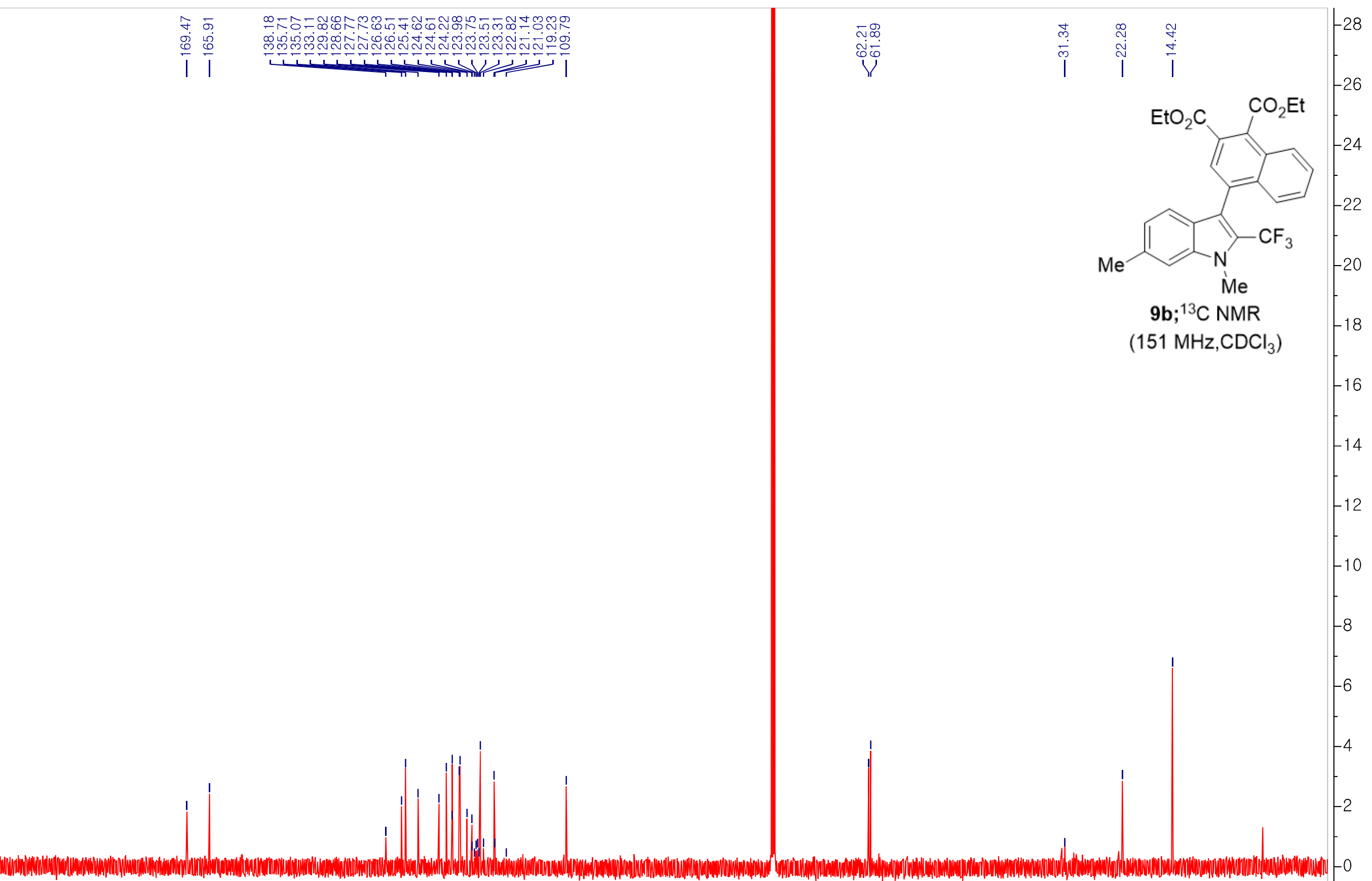

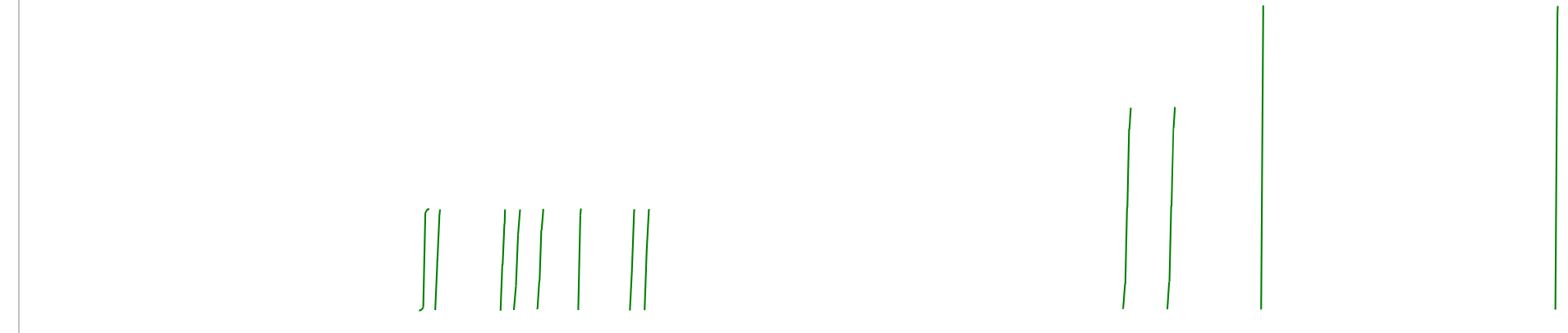

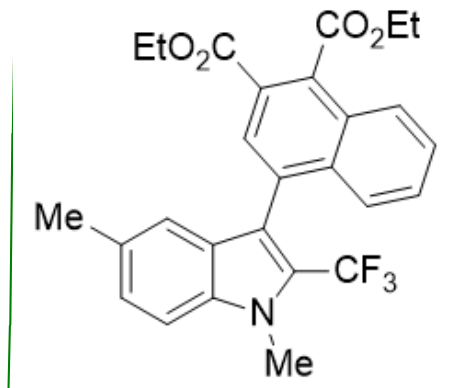

9c; ${ }^{1} \mathrm{H}$ NMR $\left(600 \mathrm{MHz}, \mathrm{CDCl}_{3}\right)$

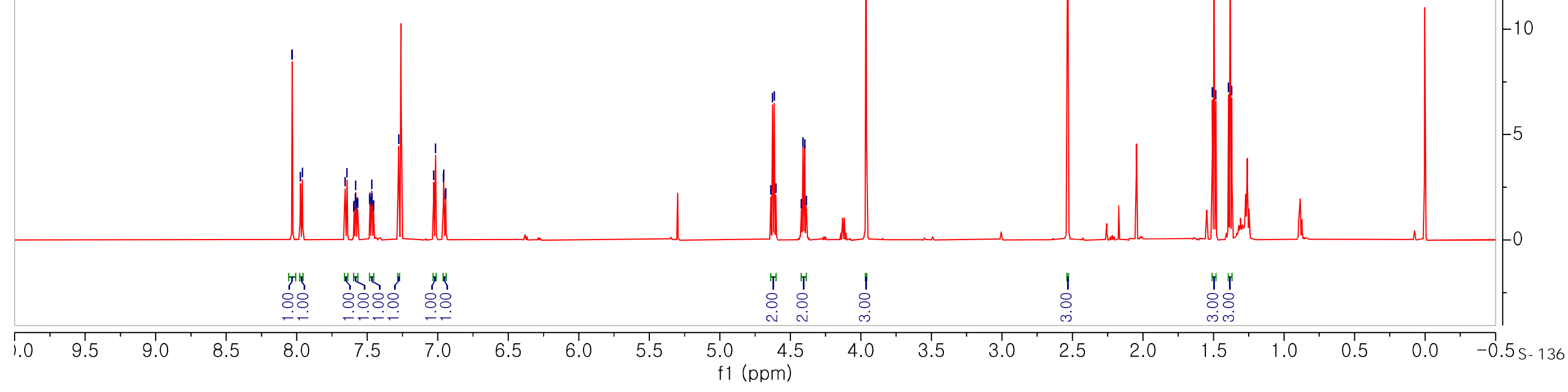




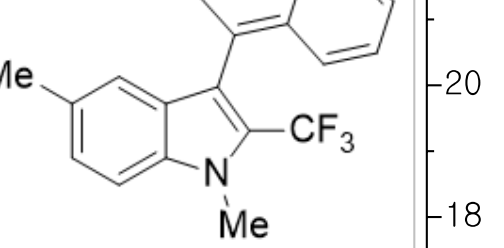

9c; ${ }^{13} \mathrm{C}$ NMR (151 MHz, $\mathrm{CDCl}_{3}$ ) 


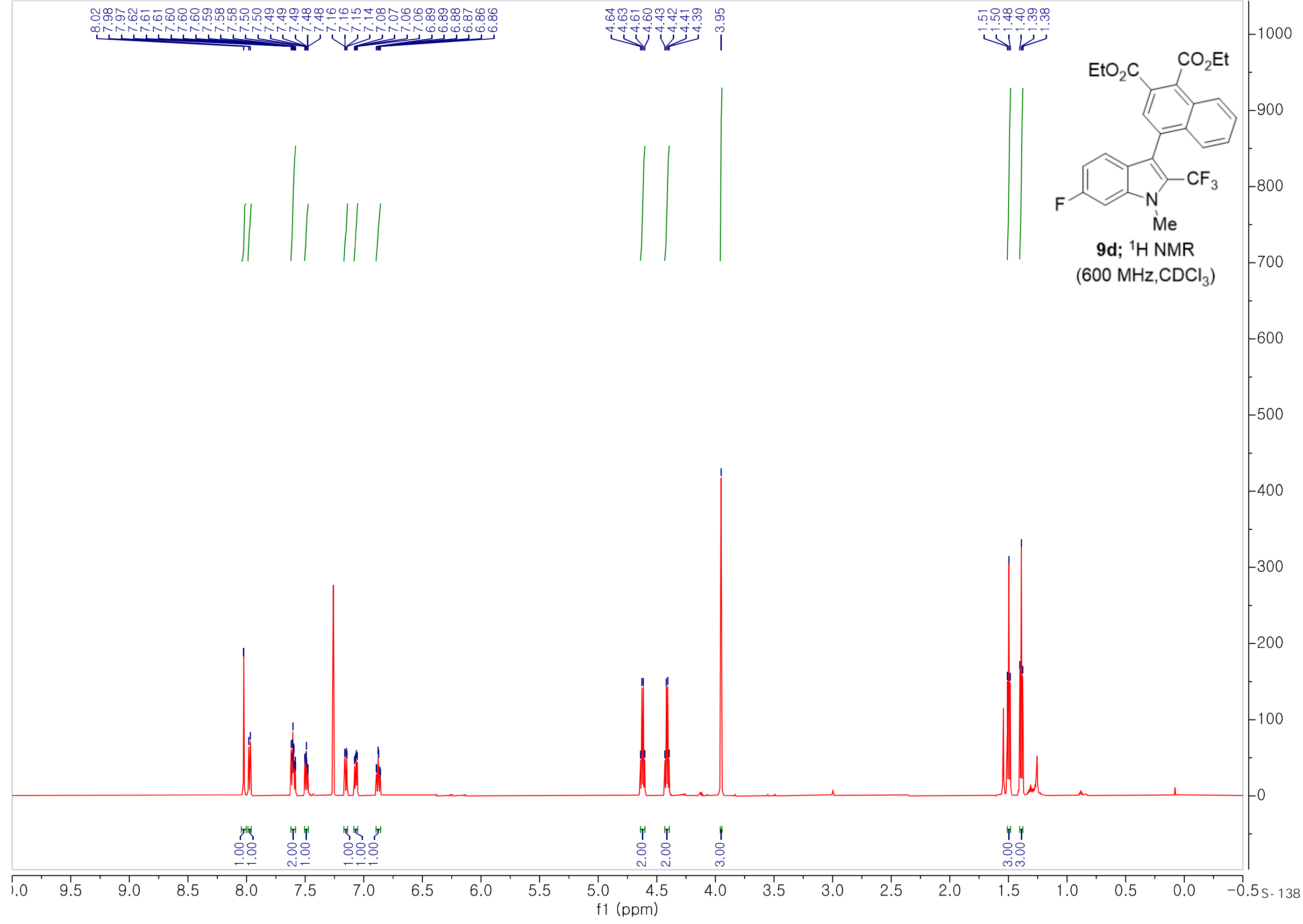



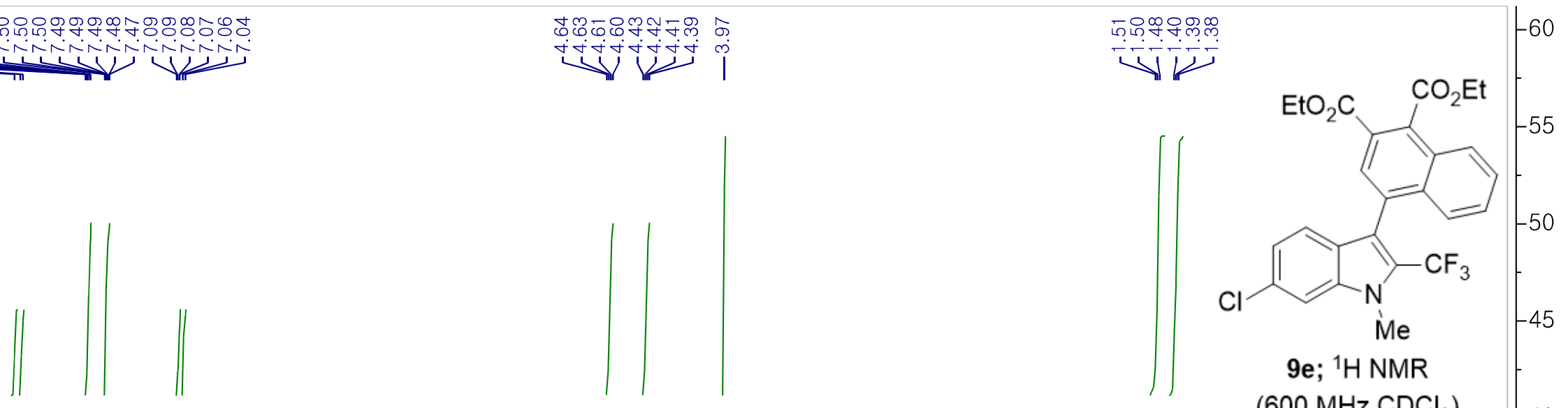

9e; ${ }^{1} \mathrm{H}$ NMR

$\left(600 \mathrm{MHz}, \mathrm{CDCl}_{3}\right.$ )

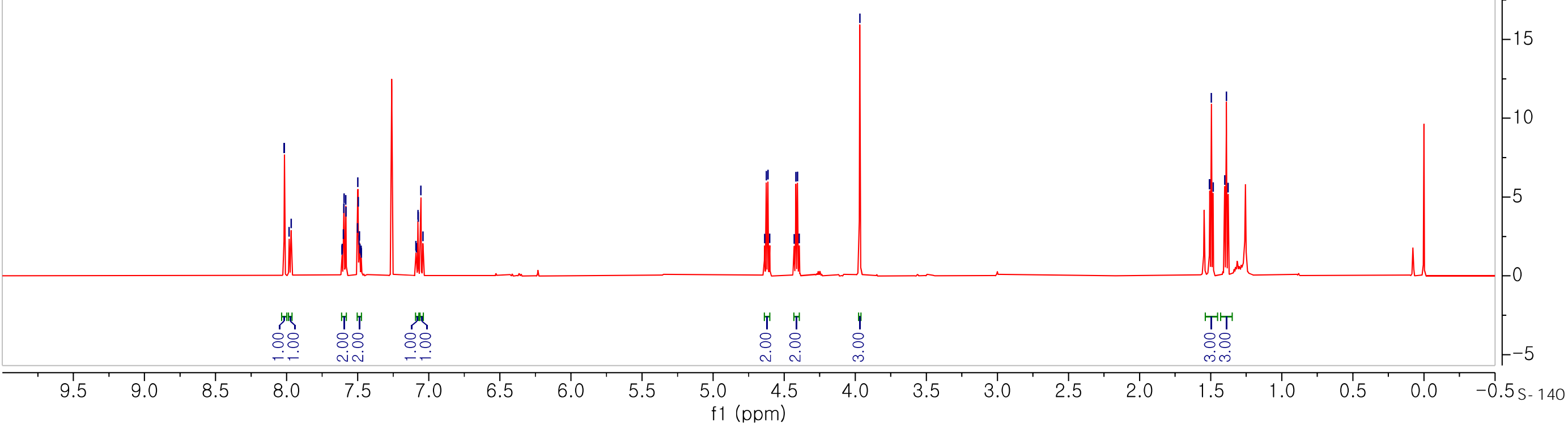


$9 \mathrm{e} ;{ }^{13} \mathrm{C}$ NMR $\left(151 \mathrm{MHz}, \mathrm{CDCl}_{3}\right)$ 